Montana

SELECTED

\title{
GEOLOGIC DATA
}

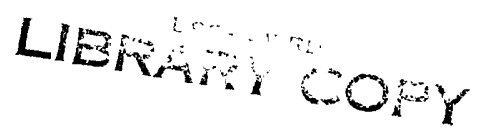

FROM THE NORTHERN GREAT PLAINS AREA OF MONTANA]

U.S. GEOLOGICAL SURVEY

Water-Resources Investigations

Open-File Report 81-415
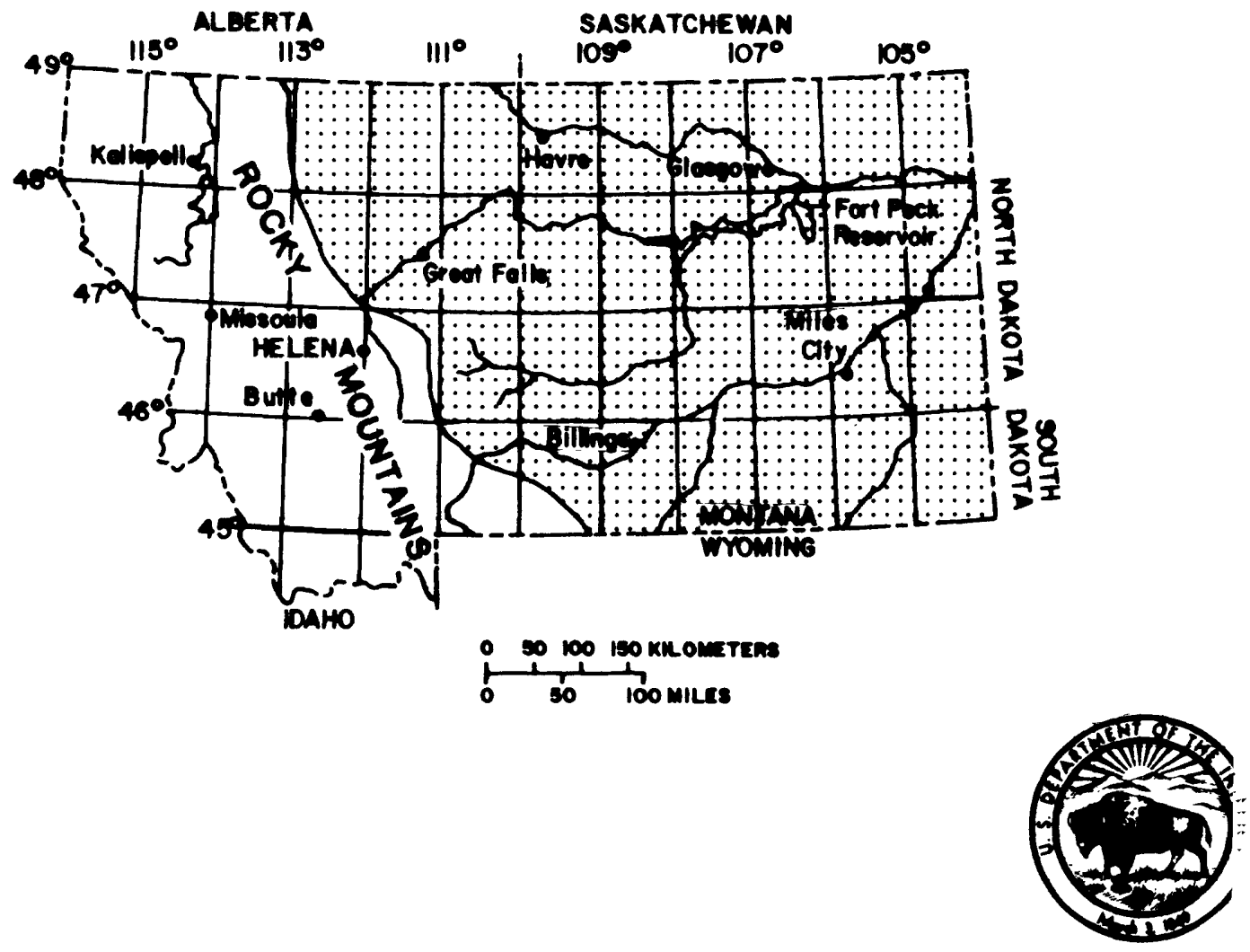
SELECTED GEOLOGIC DATA FROM THE NORTHERN

GREAT PLAINS AREA OF MONTANA

$\operatorname{Prom}$

By R. D. Feltis, B. D. Lewis, Rita L. Frasure,

Ronald P. Rioux, C. A. Jauhola, and W. R. Hotchkiss

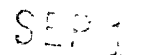

USÉs.

U.S. GEOLOGICAL SURVEY

Water-Resources Investigations

Open-File Report $81-415$

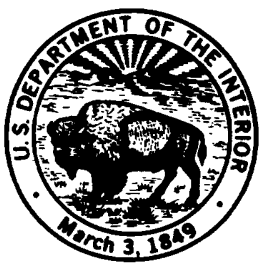

Helena, Montana

August 1981 


\section{UNITED STATES DEPARTMENT OF THE INTERIOR \\ JAMES G. WATT, Secretary GEOLOGICAL SURVEY \\ Doyle G. Federick, Acting Director}

For additional information write to:

District Chief

U.S. Geological Survey

Federal Office Building

301 South Park

Drawer 10076

Helena, MT 59626
For sale by:

Open-File Services Section Branch of Distributior

U.S. Geological Survey, MS 306

Box 25425, Denver Federal Center Denver, CO 80225

(303) 234-5888 
Abstract. . . . . . . . . . . . . . . . . . . . . 1

Introduction. . . . . . . . . . . . . . . . . . . . . . . 1

We11-numbering system . . . . . . . . . . . . . . . . . . . 2

Geologic framework. . . . . . . . . . . . . . . . . . . 3

Selected references . . . . . . . . . . . . . . . . . . . 6

Data................................. 7

\section{ILLUSTRATIONS}

Plate 1. Map showing location of selected wells used for stratigraphic correlation in the northern Great Plains of Montana . . . . In pcrket

Cover Map showing location of study area .............. Corer

Figure 1. Diagram 1llustrating well-numbering system . . . . . . . . 2

\section{TABLES}

Table 1. Stratigraphic-unit names for Montana . . . . . . . . . 4

2. Selected geologic data ............... . 7

\section{METRIC CONVERSION TABLE}

The following factors can be used to convert inch-pound units in this report to the International System (SI) of metric units.

Multiply inch-pound unit

acre

foot ( $f t$ )

mile by

4047

0.3048

1.609
To obtain SI unit:

square meter

meter

kilometer

Temperature in degrees Fahrenheit $\left({ }^{\circ} \mathrm{F}\right)$ can be converted to degrees $\operatorname{Celsius}\left({ }^{\circ} \mathrm{C}\right)$ by the formula: ${ }^{\circ} \mathrm{C}=0.556\left({ }^{\circ} \mathrm{F}-32\right)$

National Geodetic Vertical Datum of 1929 (NGVD of 1929): A geodetic datum derived from a general adjustment of the first-order level nets of both the Unf.ted States and Canada, formerly called "mean sea level." NGVD of 1929 is referred to as sea level in this report. 
THE NORTHERN GREAT PLAINS AREA OF MONTANA

by

$$
\text { R. D. Feltis, B. D. Lewis, Rita L. Frasure }
$$

Ronald P. Rioux, C. A. Jauhola, and W. R. Hotchkiss

ABSTRACT

Future water needs for development of energy resources, industry, power generation, irrigation, and domestic and municipal water supplies in the northern Great Plains have resulted in a study of the aquifers from the base of rocks of Jurassic age to land surface. This report. presents the results of correlation of regional geologic units fron geophysical well logs and geologic reports for the approximate area of Montana east of the Rocky Mountains. The altitude of the tops of 21 rocl: intervals and the composite thickness of sand in the intervals were determined for most of the 2,037 wells 1isted. The rock intervals may include one or more geologic formations. Other information 1isted in the report. includes well location, well name, height of kelly bushing above land surface, land-surface altitude, well depth, bottom-hole temperature, and age of the rock underlying the lowest rock interval penetrated by the well. A map at a scale of 1:1,000,000 shows locations of the tabulated we11s.

\section{INTRODUCTION}

Increased use of ground-water resources is likely in the northern Great PIains for the future development of energy resources, industry, power generation, irrigation, and domestic and municipal water supplies. Consequently, in 1978 the U.S. Geological Survey began a 4-year study of aquifers of Cenozoic and Mesozoic age in the northern Great Plains to define the hydrologic system, to determine availabjlity and quality of ground water, and to predict the effects of various water-use maragement plans on the entire system.

The study area for this report includes approximately all Montana east of the Rocky Mountains (see front cover). To present a complete description, rock intervals discussed herein include all aquifers and confining layers of Jurassic age or younger. Many of these aquifers have been used as a source of water only in areas where they are shallow enough to make pumping economically feasible.

The purpose of this report is to present geologic-framework data obtained by correlation of regional geologic units from geophysical well logs and geologic reports. Most geologic data have been derived from records of ofl and gas exploration wells on file at the Montana State Department of Natural Resources and Corservation and at the U.S. Geological Survey. 
In this report, locations are numbered according to geographic position within the rectangular grid system used by the U.S. Bureau of Land Management (fig. 1). The location consists of as many as 12 characters. The first three characters specify the township and its position north (N) or south (S) of the Mortana Base Line. The next three characters specify the range and its position west (W) or east (E) of the Montana Principal Meridian. The next two characters are the section number. The next one to four characters designate the quarter section (160-acre tract), quarter-quarter section (40-acre tract), quarter-quarter-quarter section (10-acre tract), and quarter-quarter-quarter-quarter section (2 1/2-acre tract), respectively, in which the well is located. The subdivisions of the section are designated $A, B, C$, and $D$ in a counterclockwise direction, beginning in the northeast quadrant. For example, as shown on figure 1, we11 35N03W27DCAB is 1ocated in the NW1/4 NE1/4 SW1/4 SE1/4 sec. 27, T. 35 N., R. 3 W.

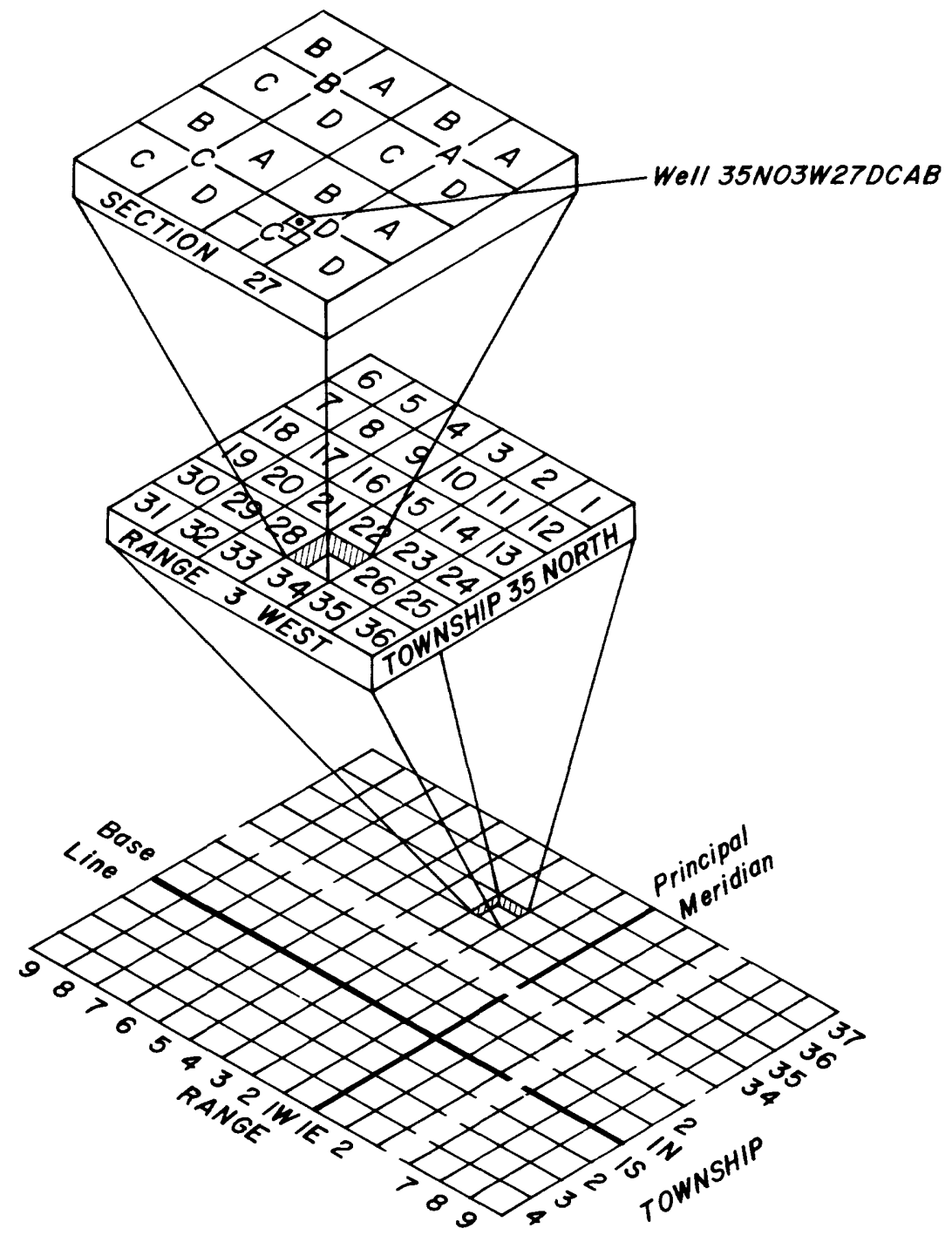

Figure 1.--We11-numbering system. 
The locations of analyzed wells ( 1 . 1) were mapped by computer using the rectangular grid system to generate latitude and longitude numbers. The program, developed by the Montana Department of Community Affairs, contains a set of latitude and longitude numbers for the four extreme corners of each township. When a rectangular grid number is entered, the program retrieves the four corners of that township and creates a regular 36-section township within those corners. Latitude and longitude numbers are then calculated for the center of the smallest subdivision of the section described. Small map discrepencies may exist where irregularly surveyed townships or sections have been represented as regular by the computer.

\section{GEOLOGIC FRAMEWORK}

Geologic-framework data were obtained from geophysical logs of oil and gas wells drilled in the Montana area of the northern Great Plains. Electrical and induction-electrical logs were used for the correlation of regional geologic units; however, in some areas only laterologs or radioactivity logs were available. Data were not available for all townships because exploratory holes had not been drilled, geophysical logs were not available, or the exploratory holes did not penetrate to the base of the Jurassic rocks in some areas. The guides for determining formation tops were boundaries used by American Stratigraphic Company, logs published in formal reports, and geologists' lithologic logs and reports for exploratory wells. An annotated bibliography of geologic and hydrologic reports of eastern Montana (Levings and others, 1981) lists published reports describing the study area.

Correlation of the formation tops and compilation of composite sand thicknesses in greater than 5-foot units were accomplished by hanging the geophysical logs in a west-to-east sequence with a spacing of one per township; about 180 miles of cross section was correlated at a time. The transgression and regression of the Cretaceous marine environments across Montana were generally oriented east-west. The coarse-grained clastic rocks thin from the source area to the west to the marine environment to the east. The cross sections immediately to the north and south were compared to maintain consistency in a north-south direction. In some problem areas, circuitous correlations were made to maintain consistency.

The diverse types of sediments deposited in continental and marine environments and in the transition areas from one environment to the other provide numerous correlation problems throughout the stratigraphic sequence. A single geophysical log can give a misconception of formation thickness and composite sand thickness of a rock unit. Regional geologic sections show the general sequence of rocks and changes in formation thicknesses but do not show local variations in thickness and extent of sedimentary deposits.

The stratigraphic-unit names within 21 rock intervals are 1 isted in table 1. A rock interval may contain one or more members, units, or formations. Several stratigraphic-unit names may be listed owing to the wide areal extent of the formation and the usage of different names in different areas. Names of local sandstone members and the U.S. Geological Survey geologic-unit codes (Rollo and others, 1975) for the most extensive units are also listed. A hydrologic designation is given as a generalized description of whether the interval functions as an aquifer, a confining layer, or a composite of aquifers and confining layers. 
Rockinterval

identification

\author{
Stratigraphic unit(s)
}

Geo-

logic

unit

code
Hydrclogic

desigration

\section{Quaternary and Tertiary}

A

B Lebo Shale Member of Fort Union Formation

C Tullock Member of Fort Union Formation

\section{Cretaceous}

$\mathrm{D}^{1} \quad$ Upper part of Hell Creek Formation

$E^{1}$ Fox Hills Sandstone and lower part of Hell Creek Formation

F

G

H Claggett Shale

I Eagle Sandstone (including Virgelle Sandstone

J Niobrara Formation and Carlile Shale

K Greenhorn Formation (including Mosby Sand-

L Belle Fourche Shale

$\mathrm{L}_{1} 2$ Mowry Shale

M Member and Shannon Sandstone Member equivalent) and Telegraph Creek Formation stone Member) or Frontier Formation

Pierre or Bearpaw Shale (including equivaMesaverde Formation)

udith River Formation (including Parkman Sandstone Member)
211JDRV

211CLGG

211EGLE

211NBRR

211GRNR

211BLFC

217MWRY

217MDDY

Aquif $\epsilon$ r

125LEBO Confiring layer

125TLCK Aquif $\in \mathrm{r}$

211HLCK Confiring layer

Aquifer

Confiring layer

Aquifer

Confining layer

Aquifer

Confining layer

Aquifer

Confining layer

Confining layer

Aquifer sandstone of subsurface usage 


\begin{tabular}{|c|c|c|c|}
\hline $\begin{array}{l}\text { Rock- } \\
\text { interval } \\
\text { identifi- } \\
\text { cation }\end{array}$ & Stratigraphic unit(s) & $\begin{array}{l}\text { Geo- } \\
\text { logic } \\
\text { unit } \\
\text { code }\end{array}$ & $\begin{array}{l}\text { Hydrologic } \\
\text { designation }\end{array}$ \\
\hline \multicolumn{4}{|c|}{ Cretaceous--continued } \\
\hline $\mathrm{N}$ & Skull Creek Shale and basal Colorado silt & $217 \mathrm{SKCK}$ & Confining layer \\
\hline 0 & $\begin{array}{l}\text { Fall River Sandstone, Dakota Sandstone, or } \\
\text { First Cat Creek sand }\end{array}$ & 217FLRV & Aquifer \\
\hline $\mathbf{P}$ & $\begin{array}{l}\text { Fuson Shale, Kootenai Formation (including } \\
\text { Second Cat Creek sand), or Cloverly } \\
\text { Formation equivalent (Kootenai Formation) }\end{array}$ & 217FUSN & Aquifer \\
\hline Q & $\begin{array}{l}\text { Lakota Formation; Third Cat Creek, Sunburst, } \\
\text { or Cut Bank Sandstone Members of Kootenai } \\
\text { Formation; or Pryor Conglomerate Member } \\
\text { of Cloverly Formation equivalent (Kootenai } \\
\text { Formation) }\end{array}$ & 217LKOT & Aquifer \\
\hline \multicolumn{4}{|c|}{ Jurassic } \\
\hline $\mathbf{R}$ & Morrison Formation & 221MRSN & Confining layer \\
\hline $\mathrm{S}$ & Swift or Sundance Formations & 221SWFT & Aquifer \\
\hline $\mathrm{T}$ & $\begin{array}{l}\text { Rierdon, Piper, Sawtooth, and Nesson } \\
\text { Formations }\end{array}$ & 221RRDN & Composite \\
\hline
\end{tabular}

1 Although the Hell Creek Formation and Fox Hills Sandstone are distinct formations, some published reports combine the lower part of the Hell Creek with the Fox Hills to form the Fox Hills-lower Hell Creek aquifer.

2 The Mowry Shale was added after the computer program for geologic units was selected for the four-state area of the northern Great Plains; therefor:, the designation $L_{1}$ is used to identify this interval. 
Geologic data for 2,037 selected wells are given in table 2. The data include land-grid location of the well, abbreviated company well name, height of kelly bushing above land surface, altitude of land surface, altitude of the top of each rock interval, composite thickness of sand in each interval, depth of wel?, bottomhole temperature, and age of the rock underlying the lowest rock interval penetrated by the well.

The data in table 2 resulted from correlation of geologic formations over a broad region. Determination of the geologic formation tops was a preliminary attempt to organize a data file that delineates rock identification f.ntervals. The geologic picks, as determined from the geophysical logs, are subjective because sample logs were not always available and the geologic tops reported by many different geologists were not always consistent. The many additional geophysical logs that are avallable can be used to determine local geologic anomalles that will not be Indicated by the data in this report.

\section{SELECTED REFERENCES}

Levings, J. F., Levings, G. W., Feltis, R. D., Hotchkiss, W. R., and Lee, R. W., 1981, Selective annotated bibliography of geology and ground-water resources in the Montana part of the northern Great Plains regional aquifer-system analysis: U.S. Geological Survey Water-Resources Investigations Open-File Report 81-401, 91 p.

Rocky Mountain Assoclation of Geologists, 1972, Geologic atlas of the Rocky Mountain region: Denver, Colo., Rocky Mountain Association of Geologists, 331 p.

Ro1lo, J. R., Price, W. E., Jr., Davidson, C. B., and Baker, C. H., Jr., 1975, Aquifer names and geologic unit codes, appendix F, of National Water Data Storage and Retrieval System, Hutchinson, N. E., compiler: U.S. Geological Survey WATSTORE User's Guide, v. 1, Open-File Report 75-426, p. F-1 to F-322.

Schlumberger Limited, 1979, Log interpretation, Vol. II--Applications: New York City, New York, Schlumberger Limited, 116 p. 
DATA

Table 2.--Selected geologic data

Interval identification: Defined in table 1.

KB FT: Height of drilling rig kelly bushing above land surface, in feet. Depths of geophysical logs are measured from the kelly bushing.

LAND ELEV FT: Altitude of land surface, in feet above sea level.

SAND FT: Composite thickness of sand, in feet, for zones greater than 5 feet thick in each interval.

TOP ELEV FT: Altitude of top of each rock interval, in feet above or belor (-) sea level. A repeated altitude number for consecutively deeper aquifers indicates that the deeper aquifer is missing from the stratigraphic section.

WELL DEPTH: In feet below land surface.

TEMP F: Bottom-hole temperature, in degrees Fahrenheit, as measured during geophysical logging.

AGE: Geologic age of the rock underlying the lowest rock interval penetrated by the well: J, Jurassic; K, Cretaceous; M, Mississippian; P, Pennsylvantan and Permian undifferentiated; $T$, Triassic. 
TABLE 2. - SELECTED GEOLUGIC DATA -- CONTINUEO

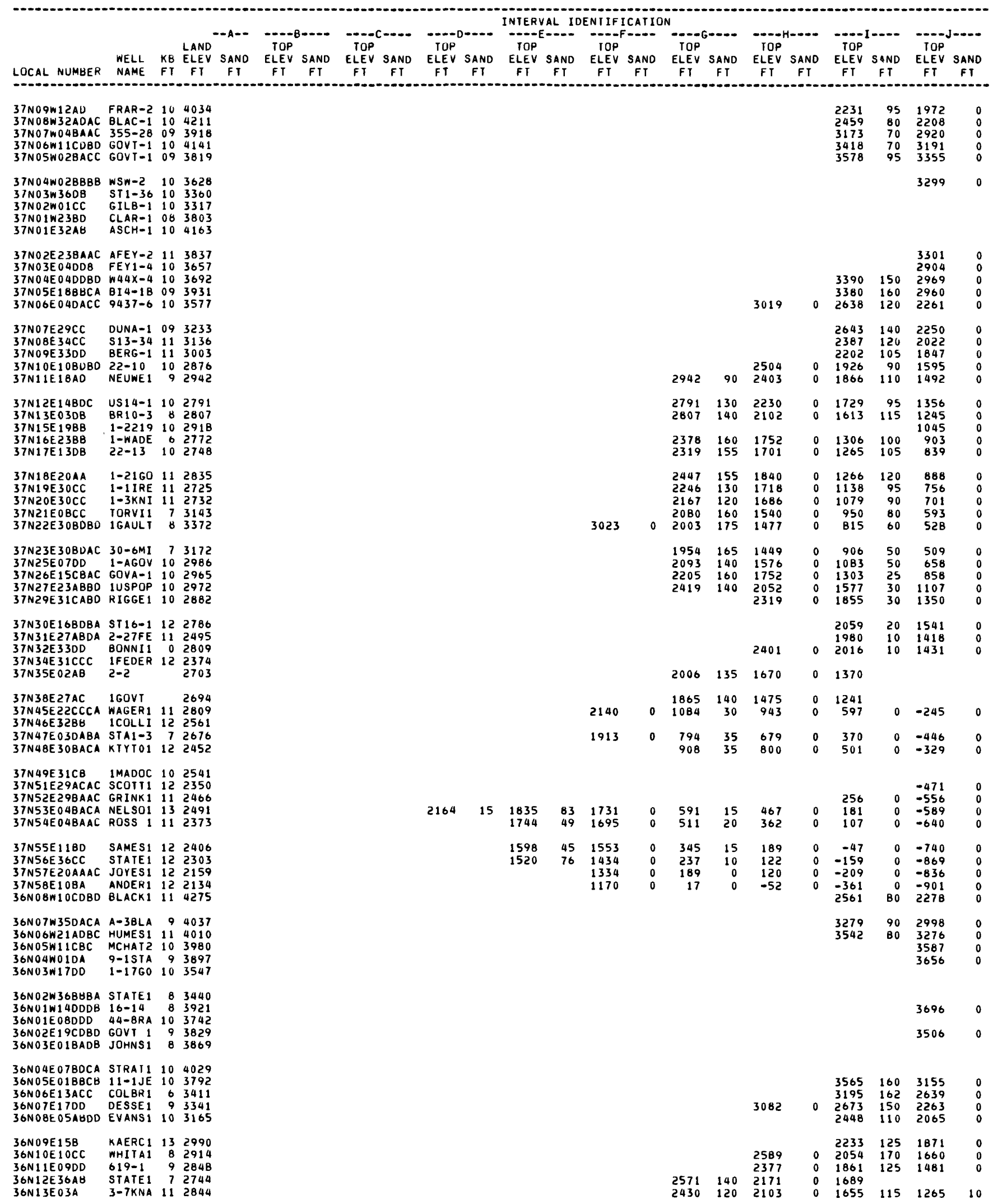


INTERVAL IDENTIFICATION

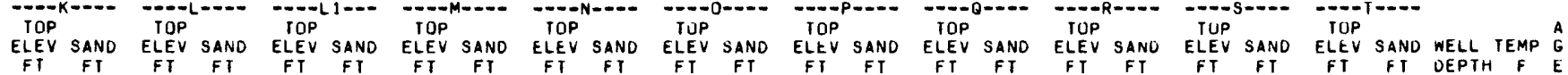

\begin{tabular}{|c|c|c|c|c|c|c|c|c|c|c|c|c|c|c|}
\hline $\begin{array}{r}916 \\
1172 \\
1910 \\
2195 \\
2363\end{array}$ & $\begin{array}{l}22 \\
30 \\
25 \\
13 \\
15\end{array}$ & $\begin{array}{r}880 \\
1129 \\
1870 \\
2148 \\
2315\end{array}$ & $\begin{array}{l}0 \\
0 \\
0\end{array}$ & $\begin{array}{r}802 \\
1061 \\
1801 \\
2050 \\
2216\end{array}$ & $\begin{array}{l}25 \\
30 \\
50 \\
35 \\
25\end{array}$ & $\begin{array}{r}664 \\
921 \\
1667 \\
1946 \\
2132\end{array}$ & $\begin{array}{r}65 \\
52 \\
55 \\
65 \\
110\end{array}$ & $\begin{array}{r}502 \\
751 \\
1491 \\
1766 \\
1924\end{array}$ & $\begin{array}{l}75 \\
70 \\
90 \\
80 \\
70\end{array}$ & $\begin{array}{r}234 \\
489 \\
1193 \\
1495 \\
1622\end{array}$ & $\begin{array}{l}24 \\
30 \\
35 \\
14 \\
17\end{array}$ & $\begin{array}{r}208 \\
454 \\
1158 \\
1481 \\
1605\end{array}$ & $\begin{array}{r}150 \\
154 \\
130 \\
148 \\
85\end{array}$ & $\begin{array}{r}-403 \\
-169 \\
619 \\
899 \\
1052\end{array}$ \\
\hline $\begin{array}{l}2323 \\
2764 \\
2471 \\
2542 \\
3403\end{array}$ & $\begin{array}{l}17 \\
25 \\
30 \\
30 \\
22\end{array}$ & $\begin{array}{l}2268 \\
2698 \\
2405 \\
2470 \\
3345\end{array}$ & 0 & $\begin{array}{l}2181 \\
2624 \\
2327 \\
2394 \\
3270\end{array}$ & $\begin{array}{l}33 \\
31 \\
28 \\
40 \\
41\end{array}$ & $\begin{array}{l}2070 \\
2507 \\
2199 \\
2246 \\
3109\end{array}$ & $\begin{array}{r}60 \\
90 \\
105 \\
105 \\
110\end{array}$ & $\begin{array}{l}1872 \\
2342 \\
1930 \\
2027 \\
2908\end{array}$ & $\begin{array}{r}80 \\
115 \\
50 \\
65 \\
65\end{array}$ & $\begin{array}{l}1578 \\
1987 \\
1697 \\
1766 \\
2630\end{array}$ & $\begin{array}{l}10 \\
20 \\
30 \\
20 \\
25\end{array}$ & $\begin{array}{l}1568 \\
1965 \\
1665 \\
1739 \\
2598\end{array}$ & $\begin{array}{r}80 \\
110 \\
125 \\
85 \\
50\end{array}$ & $\begin{array}{l}1202 \\
1535 \\
1300 \\
1333 \\
2301\end{array}$ \\
\hline $\begin{array}{l}2479 \\
2112 \\
2209 \\
2213 \\
1524\end{array}$ & $\begin{array}{l}20 \\
25 \\
30 \\
26 \\
35\end{array}$ & $\begin{array}{l}2424 \\
2048 \\
2146 \\
2152 \\
1465\end{array}$ & & $\begin{array}{l}2344 \\
1951 \\
2048 \\
2060 \\
1333\end{array}$ & $\begin{array}{l}46 \\
30 \\
40 \\
42 \\
41\end{array}$ & $\begin{array}{l}2154 \\
1747 \\
1833 \\
1812 \\
1064\end{array}$ & $\begin{array}{r}90 \\
90 \\
115 \\
130 \\
90\end{array}$ & $\begin{array}{r}1967 \\
1578 \\
1642 \\
1640 \\
951\end{array}$ & $\begin{array}{r}55 \\
55 \\
100 \\
100 \\
45\end{array}$ & $\begin{array}{r}1619 \\
1317 \\
1340 \\
1364 \\
657\end{array}$ & $\begin{array}{l}15 \\
35 \\
15 \\
16 \\
11\end{array}$ & $\begin{array}{r}1604 \\
1268 \\
1320 \\
1340 \\
646\end{array}$ & $\begin{array}{r}75 \\
70 \\
85 \\
110 \\
25\end{array}$ & $\begin{array}{r}1187 \\
777 \\
1006 \\
993 \\
317\end{array}$ \\
\hline $\begin{array}{r}1520 \\
1322 \\
1169 \\
906 \\
811\end{array}$ & $\begin{array}{l}25 \\
25 \\
40 \\
30 \\
35\end{array}$ & $\begin{array}{r}1460 \\
1244 \\
1081 \\
834 \\
743\end{array}$ & & $\begin{array}{r}1322 \\
1110 \\
949 \\
684 \\
597\end{array}$ & $\begin{array}{l}53 \\
41 \\
47 \\
50 \\
40\end{array}$ & $\begin{array}{r}1036 \\
806 \\
627 \\
367 \\
291\end{array}$ & $\begin{array}{l}95 \\
58 \\
85 \\
85 \\
90\end{array}$ & $\begin{array}{l}926 \\
687 \\
496 \\
226 \\
133\end{array}$ & $\begin{array}{r}90 \\
105 \\
90 \\
40 \\
35\end{array}$ & $\begin{array}{r}550 \\
327 \\
174 \\
-96 \\
-152\end{array}$ & $\begin{array}{l}17 \\
12 \\
20 \\
30 \\
30\end{array}$ & $\begin{array}{r}533 \\
309 \\
152 \\
-126 \\
-182\end{array}$ & $\begin{array}{r}60 \\
76 \\
110 \\
25 \\
50\end{array}$ & $\begin{array}{r}268 \\
-15 \\
-175 \\
-383 \\
-445\end{array}$ \\
\hline $\begin{array}{l}657 \\
564 \\
421 \\
312 \\
253\end{array}$ & $\begin{array}{l}30 \\
20 \\
25 \\
24 \\
30\end{array}$ & $\begin{array}{l}588 \\
504 \\
325 \\
233 \\
172\end{array}$ & & $\begin{array}{l}439 \\
345\end{array}$ & $\begin{array}{l}35 \\
40\end{array}$ & $\begin{array}{r}123 \\
14 \\
-142 \\
-303 \\
-372\end{array}$ & $\begin{array}{l}80 \\
85 \\
95 \\
60 \\
72\end{array}$ & $\begin{array}{r}-39 \\
-287 \\
-420 \\
-486\end{array}$ & $\begin{array}{r}30 \\
0 \\
0 \\
259\end{array}$ & $\begin{array}{l}-322 \\
-582 \\
-741 \\
-794\end{array}$ & $\begin{array}{l}34 \\
55 \\
39 \\
31\end{array}$ & $\begin{array}{l}-356 \\
-643 \\
-780 \\
-859\end{array}$ & $\begin{array}{l}50 \\
40 \\
24 \\
20\end{array}$ & $\begin{array}{l}-575 \\
-815 \\
-907 \\
-982\end{array}$ \\
\hline $\begin{array}{r}274 \\
169 \\
117 \\
28 \\
-20\end{array}$ & $\begin{array}{l}20 \\
20 \\
20 \\
30 \\
30\end{array}$ & $\begin{array}{r}225 \\
109 \\
49 \\
-36 \\
-75\end{array}$ & & $\begin{array}{r}10 \\
-108 \\
-163 \\
-262 \\
-208\end{array}$ & $\begin{array}{l}25 \\
22 \\
25\end{array}$ & $\begin{array}{l}-497 \\
-583 \\
-644\end{array}$ & $\begin{array}{r}90 \\
110 \\
75 \\
70 \\
55\end{array}$ & $\begin{array}{l}-474 \\
-574 \\
-629 \\
-700 \\
-752\end{array}$ & $\begin{array}{l}40 \\
61 \\
58 \\
60 \\
65\end{array}$ & $\begin{array}{r}-773 \\
-879 \\
-945 \\
-1002 \\
-1068\end{array}$ & $\begin{array}{l}20 \\
20 \\
20 \\
21 \\
22\end{array}$ & $\begin{array}{r}-808 \\
-916 \\
-980 \\
-1023 \\
-1090\end{array}$ & $\begin{array}{l}15 \\
20 \\
15 \\
15 \\
15\end{array}$ & $\begin{array}{r}-934 \\
-1036 \\
-1117 \\
-1137 \\
-1208\end{array}$ \\
\hline $\begin{array}{l}-28 \\
136 \\
335 \\
592 \\
840\end{array}$ & $\begin{array}{l}20 \\
15 \\
15 \\
18 \\
16\end{array}$ & $\begin{array}{r}-90 \\
82 \\
280 \\
541 \\
794\end{array}$ & & $\begin{array}{r}-324 \\
-172 \\
22 \\
278 \\
541\end{array}$ & $\begin{array}{l}30 \\
20 \\
22 \\
25 \\
22\end{array}$ & $\begin{array}{r}-681 \\
-564 \\
-362 \\
-108 \\
138\end{array}$ & $\begin{array}{l}70 \\
50 \\
30 \\
20 \\
30\end{array}$ & $\begin{array}{r}-765 \\
-647 \\
-440 \\
-188 \\
60\end{array}$ & $\begin{array}{r}72 \\
87 \\
91 \\
98 \\
102\end{array}$ & $\begin{array}{r}-1101 \\
-966 \\
-756 \\
-508 \\
-240\end{array}$ & $\begin{array}{l}10 \\
28 \\
30 \\
15 \\
29\end{array}$ & $\begin{array}{r}-1129 \\
-994 \\
-787 \\
-550 \\
-269\end{array}$ & $\begin{array}{r}10 \\
10 \\
10 \\
0 \\
0\end{array}$ & $\begin{array}{r}-1259 \\
-1099 \\
-860 \\
-580 \\
-342\end{array}$ \\
\hline $\begin{array}{r}1033 \\
906 \\
927\end{array}$ & $\begin{array}{l}15 \\
15 \\
15\end{array}$ & $\begin{array}{l}928 \\
863 \\
871\end{array}$ & & $\begin{array}{l}728 \\
621 \\
631\end{array}$ & $\begin{array}{l}16 \\
16 \\
13\end{array}$ & $\begin{array}{l}315 \\
205 \\
233\end{array}$ & $\begin{array}{l}25 \\
65 \\
10\end{array}$ & $\begin{array}{r}236 \\
78 \\
125\end{array}$ & $\begin{array}{l}114 \\
114 \\
112\end{array}$ & $\begin{array}{r}-72 \\
-182 \\
-124\end{array}$ & $\begin{array}{l}25 \\
25 \\
33\end{array}$ & $\begin{array}{r}-97 \\
-221 \\
-166\end{array}$ & $\begin{array}{r}10 \\
12 \\
0\end{array}$ & $\begin{array}{l}-182 \\
-334 \\
-219\end{array}$ \\
\hline
\end{tabular}

65
61
65
65
50
52
77
77
25
65
20
53
53
14
18
37
30
40
55
40
40
28
10
31
21
21
24
24
3

$\begin{array}{lllllll}-484 & 0 & -520 & 0 & 4780 & 85 & M\end{array}$ 65 52 (28 1 5 5 0 8 25 60

.

\begin{tabular}{|c|c|c|c|c|c|c|c|c|c|c|}
\hline-612 & 15 & -675 & 0 & -820 & 0 & -1155 & 0 & $\begin{array}{r}-538 \\
-1160 \\
-1266\end{array}$ & $\begin{array}{l}137 \\
139 \\
118\end{array}$ & $\begin{array}{r}-885 \\
-1458 \\
-1545\end{array}$ \\
\hline $\begin{array}{l}-820 \\
-708\end{array}$ & $\begin{array}{l}25 \\
40\end{array}$ & $\begin{array}{l}-869 \\
-774\end{array}$ & $\begin{array}{l}0 \\
0\end{array}$ & $\begin{array}{r}-1008 \\
-907\end{array}$ & $\begin{array}{l}0 \\
0\end{array}$ & $\begin{array}{l}-1327 \\
-1217\end{array}$ & $\begin{array}{l}0 \\
0\end{array}$ & $\begin{array}{l}-1339 \\
-1228\end{array}$ & $\begin{array}{r}100 \\
93\end{array}$ & $\begin{array}{l}-1593 \\
-1482\end{array}$ \\
\hline $\begin{array}{l}-819 \\
-900 \\
-919 \\
-970\end{array}$ & $\begin{array}{l}30 \\
25 \\
30 \\
25\end{array}$ & $\begin{array}{r}-880 \\
-967 \\
-982 \\
-1037\end{array}$ & $\begin{array}{l}0 \\
0 \\
0 \\
0\end{array}$ & 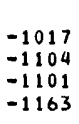 & $\begin{array}{r}0 \\
0 \\
0 \\
10\end{array}$ & $\begin{array}{l}-1301 \\
-1365 \\
-1336 \\
-1368\end{array}$ & $\begin{array}{r}0 \\
10 \\
33 \\
45\end{array}$ & $\begin{array}{l}-1194 \\
-1481 \\
-1381 \\
-1369 \\
-1413\end{array}$ & $\begin{array}{r}108 \\
89 \\
108 \\
103 \\
119\end{array}$ & $\begin{array}{l}-1469 \\
-1570 \\
-1688 \\
-1657 \\
-1707\end{array}$ \\
\hline $\begin{array}{r}-1105 \\
-1226 \\
-1188 \\
-1267 \\
1236\end{array}$ & $\begin{array}{l}15 \\
15 \\
15 \\
20 \\
28\end{array}$ & $\begin{array}{r}-1185 \\
-1323 \\
-1283 \\
-1363 \\
1184\end{array}$ & $\begin{array}{l}0 \\
0 \\
0 \\
0 \\
0\end{array}$ & $\begin{array}{r}-1319 \\
-1469 \\
-1423 \\
-1511 \\
1120\end{array}$ & $\begin{array}{r}10 \\
0 \\
0 \\
0 \\
34\end{array}$ & $\begin{array}{r}-1519 \\
-1647 \\
-1617 \\
-1671 \\
956\end{array}$ & $\begin{array}{l}43 \\
10 \\
10 \\
45 \\
40\end{array}$ & $\begin{array}{r}-1562 \\
-1684 \\
-1659 \\
-1734 \\
831\end{array}$ & $\begin{array}{r}131 \\
140 \\
99 \\
111 \\
65\end{array}$ & $\begin{array}{r}-1867 \\
-1985 \\
-1909 \\
-1978 \\
545\end{array}$ \\
\hline $\begin{array}{l}1974 \\
2278 \\
2658 \\
2733 \\
2859\end{array}$ & $\begin{array}{l}20 \\
16 \\
14 \\
17 \\
23\end{array}$ & $\begin{array}{l}1926 \\
2246 \\
2628 \\
2689 \\
2805\end{array}$ & $\begin{array}{l}0 \\
0 \\
0 \\
0 \\
0\end{array}$ & $\begin{array}{l}1876 \\
2171 \\
2538 \\
2617 \\
2741\end{array}$ & $\begin{array}{l}35 \\
65 \\
36 \\
24 \\
35\end{array}$ & $\begin{array}{l}1724 \\
2024 \\
2418 \\
2500 \\
2619\end{array}$ & $\begin{array}{r}45 \\
40 \\
100 \\
100 \\
115\end{array}$ & $\begin{array}{l}1596 \\
1873 \\
2195 \\
2300 \\
2427\end{array}$ & $\begin{array}{r}90 \\
55 \\
65 \\
90 \\
150\end{array}$ & $\begin{array}{l}1293 \\
1574 \\
1938 \\
1986 \\
2060\end{array}$ \\
\hline $\begin{array}{l}3085 \\
2834 \\
2957 \\
2685 \\
2783\end{array}$ & $\begin{array}{l}20 \\
25 \\
28 \\
27 \\
22\end{array}$ & $\begin{array}{l}3045 \\
2776 \\
2906 \\
2616 \\
2724\end{array}$ & $\begin{array}{l}0 \\
0 \\
0 \\
0 \\
0\end{array}$ & $\begin{array}{l}2963 \\
2693 \\
2817 \\
2529 \\
2633\end{array}$ & $\begin{array}{l}25 \\
36 \\
34 \\
37 \\
40\end{array}$ & $\begin{array}{l}2818 \\
2559 \\
2674 \\
2400 \\
2384\end{array}$ & $\begin{array}{r}115 \\
85 \\
85 \\
65 \\
70\end{array}$ & $\begin{array}{l}2593 \\
2337 \\
2462 \\
2190 \\
2267\end{array}$ & $\begin{array}{l}80 \\
55 \\
25 \\
70 \\
75\end{array}$ & $\begin{array}{l}2318 \\
2041 \\
2191 \\
1882 \\
1924\end{array}$ \\
\hline $\begin{array}{l}2902 \\
2431 \\
2047 \\
1527 \\
1343\end{array}$ & $\begin{array}{l}21 \\
15 \\
20 \\
30 \\
20\end{array}$ & $\begin{array}{l}2846 \\
2353 \\
1996 \\
1477 \\
1287\end{array}$ & $\begin{array}{l}0 \\
0 \\
0 \\
0 \\
0\end{array}$ & $\begin{array}{l}2756 \\
2149 \\
1867 \\
1345 \\
1155\end{array}$ & $\begin{array}{l}50 \\
37 \\
38 \\
37 \\
35\end{array}$ & $\begin{array}{r}2512 \\
2006 \\
1607 \\
1067 \\
855\end{array}$ & $\begin{array}{r}90 \\
105 \\
90 \\
70 \\
90\end{array}$ & $\begin{array}{r}2364 \\
1885 \\
1475 \\
948 \\
740\end{array}$ & $\begin{array}{r}95 \\
100 \\
95 \\
105 \\
120\end{array}$ & $\begin{array}{r}2057 \\
1554 \\
1347 \\
608 \\
385\end{array}$ \\
\hline $\begin{array}{r}1160 \\
948 \\
783\end{array}$ & $\begin{array}{l}33 \\
35 \\
15\end{array}$ & $\begin{array}{r}1105 \\
883 \\
712\end{array}$ & $\begin{array}{l}0 \\
0 \\
0\end{array}$ & $\begin{array}{l}973 \\
742 \\
566\end{array}$ & $\begin{array}{r}40 \\
20 \\
0\end{array}$ & $\begin{array}{l}658 \\
424 \\
247\end{array}$ & $\begin{array}{l}70 \\
45 \\
20\end{array}$ & $\begin{array}{r}521 \\
282 \\
93\end{array}$ & $\begin{array}{r}115 \\
45 \\
25\end{array}$ & $\begin{array}{r}131 \\
-20 \\
-194\end{array}$ \\
\hline 587 & 20 & 496 & 0 & 345 & 0 & 18 & 25 & & & \\
\hline
\end{tabular}

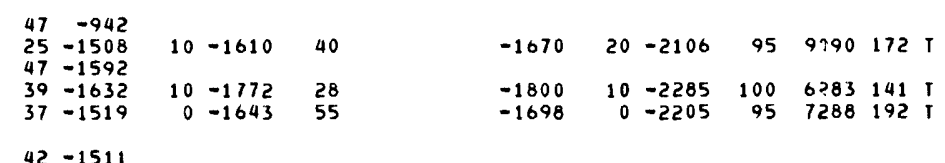

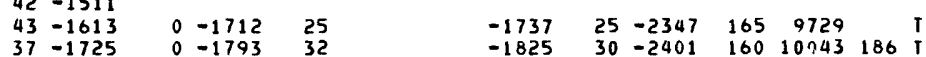

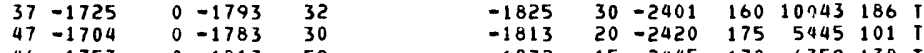

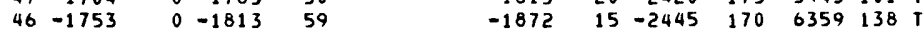

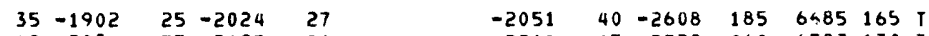

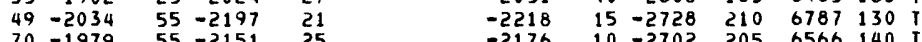

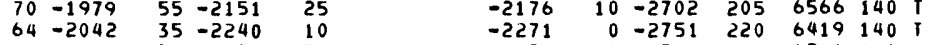
$\begin{array}{rrrrrrrrrrr}64 & -2042 & 35 & -2240 & 10 & -2271 & 0 & -2751 & 220 & 6419 & 140 \\ 35 & 504 & 135 & -136 & 54 & -190 & 0 & -211 & 7 & 4716 & 116 \mathrm{M}\end{array}$

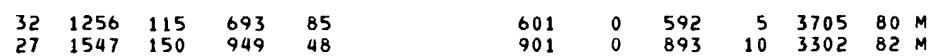

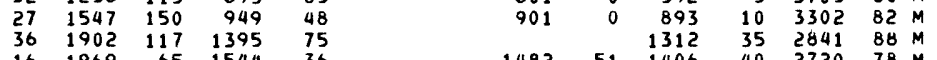

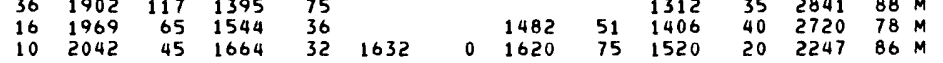

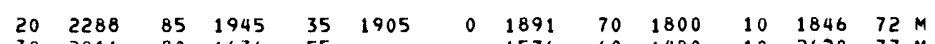

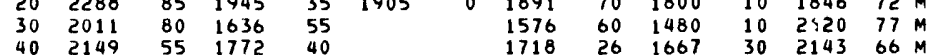

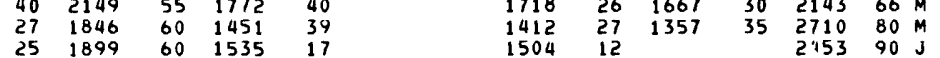
$\begin{array}{llllllllllll}15 & 2042 & 80 & 1676 & 10 & 1659 & 10 & 1619 & 30 & 2339 & 77 \mathrm{M}\end{array}$

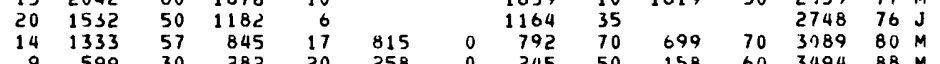

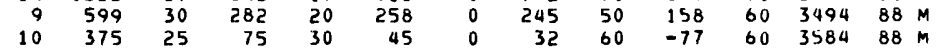
$\begin{array}{lllllllllll}21 & 110 & 50 & -154 & 25 & -197 & 50 & -270 & 95 & 3552 & 111 \\ 1\end{array}$ $\begin{array}{lllllll}-335 & 20 & -436 & 140 & 3719 & 105 & M \\ -520 & 20 & -625 & 125 & 3796 & 99 & M\end{array}$ $2770 \quad 82 K$ 
TABLE 2. - SELECTED GEOLOGIC DATA - - CONIINUED

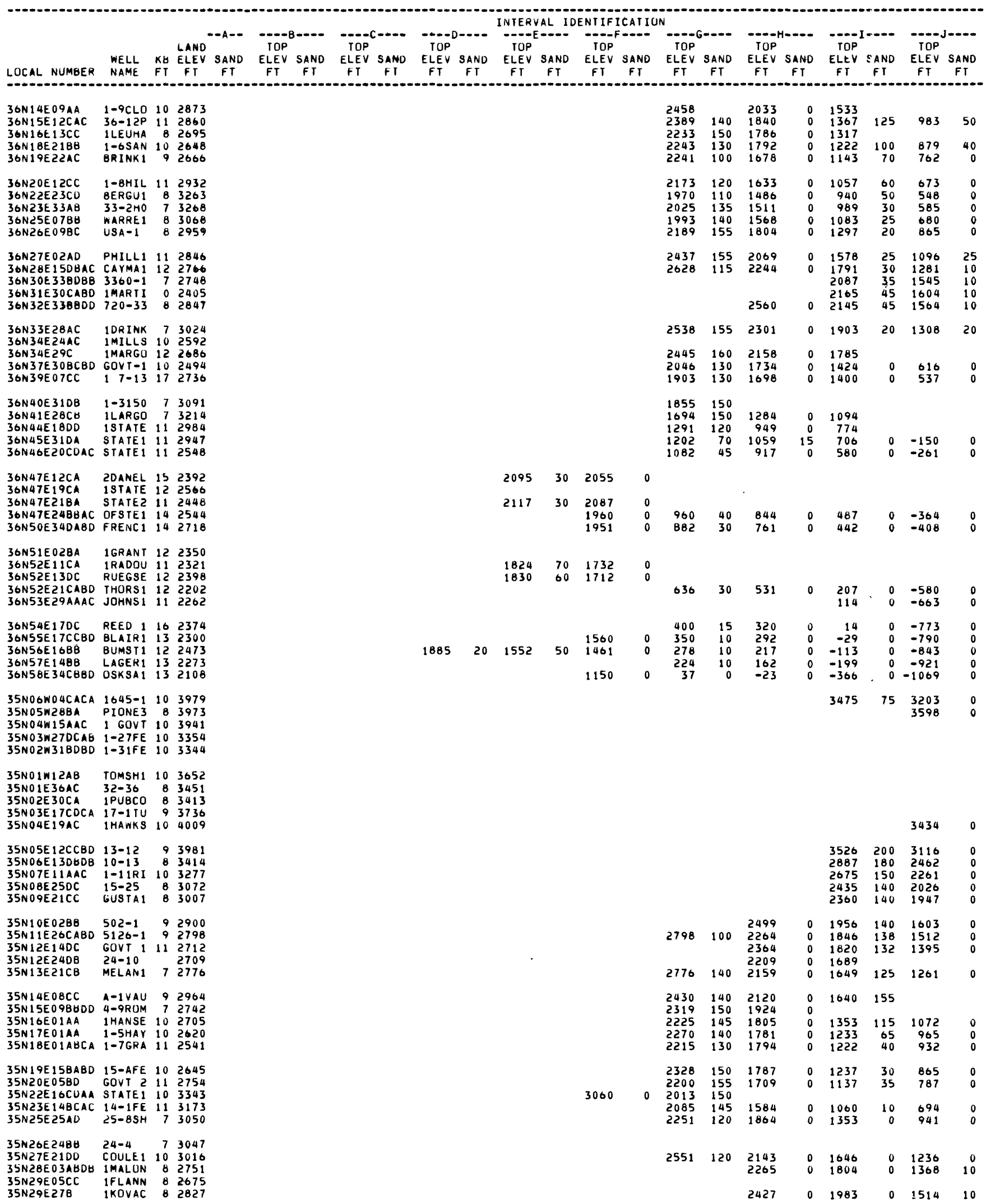




\begin{tabular}{|c|c|c|c|c|c|c|c|c|c|c|c|c|c|c|c|c|c|c|c|c|c|c|c|c|}
\hline $\begin{array}{l}-\cdots-K \\
\text { TOP } \\
\text { ELEV }\end{array}$ & $\begin{array}{l}\text { SAND } \\
\text { FT }\end{array}$ & $\begin{array}{l}\text { TOP } \\
\text { ELEV } \\
\text { FT }\end{array}$ & $\begin{array}{l}\text { SAND } \\
\text { FT }\end{array}$ & $\begin{array}{l}\text { TOP } \\
\text { ELEV } \\
\text { FT }\end{array}$ & $\begin{array}{l}\text { SAND } \\
\text { FT }\end{array}$ & $\begin{array}{l}---M \\
\text { TOP } \\
\text { ELEV } \\
\text { FT }\end{array}$ & SAND & $\begin{array}{l}\text { TOP } \\
\text { ELEV } \\
\text { FY }\end{array}$ & $\begin{array}{l}\text { SAND } \\
\text { FT }\end{array}$ & \multicolumn{2}{|c|}{$\begin{array}{l}\text { TOP } \\
\text { ELEV SAND } \\
\text { FT FT }\end{array}$} & $\begin{array}{l}\text { TUP } \\
\text { ELEV } \\
F Y\end{array}$ & $\begin{array}{l}\text { SAND } \\
\text { FT }\end{array}$ & $\begin{array}{l}\text { IUP } \\
\text { ELEV } \\
\text { FT }\end{array}$ & $\begin{array}{l}\text { SAND } \\
\text { FT }\end{array}$ & \multicolumn{2}{|c|}{$\begin{array}{l}\text { IOP } \\
\text { ELEV SAND } \\
\text { FT FT }\end{array}$} & \multicolumn{2}{|c|}{$\begin{array}{l}\text { TUP } \\
\text { ELEV SAND } \\
\text { FT FI }\end{array}$} & $\begin{array}{c}---T \\
\text { TUP } \\
\text { ELEV } \\
\text { FT }\end{array}$ & $\begin{array}{l}\text { SAND } \\
\text { FT }\end{array}$ & \multicolumn{3}{|c|}{$\begin{array}{l}\text { WTLL TEMP } \\
\text { UFPTH F }\end{array}$} \\
\hline 361 & 30 & 276 & 0 & 112 & 10 & -237 & 30 & -360 & 33 & -652 & 0 & -694 & 15 & -891 & 16 & & & -907 & 0 & -1045 & 125 & 6297 & 126 & $m$ \\
\hline $\begin{array}{l}228 \\
172\end{array}$ & $\begin{array}{l}15 \\
15\end{array}$ & $\begin{array}{l}164 \\
105\end{array}$ & $\begin{array}{l}10 \\
10\end{array}$ & $\begin{array}{r}-42 \\
-107\end{array}$ & $\begin{array}{l}0 \\
0\end{array}$ & $\begin{array}{l}-393 \\
-465\end{array}$ & $\begin{array}{l}60 \\
60\end{array}$ & $\begin{array}{l}-514 \\
-583\end{array}$ & $\begin{array}{l}47 \\
64\end{array}$ & $\begin{array}{l}-820 \\
-885\end{array}$ & $\begin{array}{l}35 \\
25\end{array}$ & $\begin{array}{l}-862 \\
-915\end{array}$ & $\begin{array}{l}20 \\
10\end{array}$ & $\begin{array}{l}-1028 \\
-1078\end{array}$ & $\begin{array}{l}54 \\
15\end{array}$ & & & $\begin{array}{l}-1082 \\
-1099\end{array}$ & $\begin{array}{r}0 \\
40\end{array}$ & & & $\begin{array}{l}4177 \\
4216\end{array}$ & $\begin{array}{l}109 \\
102\end{array}$ & $\mathrm{~J}$ \\
\hline $\begin{array}{r}110 \\
-2 \\
15 \\
104 \\
307\end{array}$ & $\begin{array}{r}15 \\
15 \\
15 \\
0 \\
10\end{array}$ & $\begin{array}{r}27 \\
-89 \\
-50 \\
18 \\
222\end{array}$ & $\begin{array}{r}10 \\
10 \\
10 \\
0 \\
0\end{array}$ & $\begin{array}{r}-178 \\
-283 \\
-244 \\
-164 \\
35\end{array}$ & $\begin{array}{l}0 \\
0 \\
0 \\
0 \\
0\end{array}$ & $\begin{array}{l}-515 \\
-632 \\
-605 \\
-542 \\
-353\end{array}$ & $\begin{array}{r}120 \\
50 \\
40 \\
30 \\
35\end{array}$ & $\begin{array}{l}-640 \\
-736 \\
=715 \\
-603 \\
-416\end{array}$ & $\begin{array}{l}60 \\
71 \\
79 \\
83 \\
90\end{array}$ & $\begin{array}{r}-942 \\
-1053 \\
-1038 \\
-946 \\
-753\end{array}$ & $\begin{array}{r}0 \\
0 \\
0 \\
20 \\
10\end{array}$ & $\begin{array}{r}-985 \\
-1090 \\
-1081 \\
-1003 \\
-795\end{array}$ & $\begin{array}{l}35 \\
15 \\
0 \\
25 \\
15\end{array}$ & $\begin{array}{r}-1101 \\
-1232 \\
-1211 \\
-1127 \\
-900\end{array}$ & $\begin{array}{l}58 \\
10 \\
31 \\
17 \\
10\end{array}$ & & & $\begin{array}{r}-1159 \\
-1269 \\
-1242 \\
-1144 \\
-925\end{array}$ & $\begin{array}{l}55 \\
20 \\
15 \\
20 \\
0\end{array}$ & $\begin{array}{l}-1443 \\
-1367 \\
-1174\end{array}$ & $\begin{array}{l}80 \\
90 \\
75\end{array}$ & $\begin{array}{l}4696 \\
5081 \\
5048 \\
4877 \\
4620\end{array}$ & $\begin{array}{r}103 \\
107 \\
112 \\
110 \\
98\end{array}$ & $\begin{array}{l}\mathrm{J} \\
\mathrm{M} \\
\mathrm{M} \\
\mathrm{M}\end{array}$ \\
\hline $\begin{array}{r}573 \\
770 \\
1025 \\
1095 \\
1055\end{array}$ & $\begin{array}{r}0 \\
0 \\
10 \\
20 \\
15\end{array}$ & $\begin{array}{l}464 \\
642\end{array}$ & $\begin{array}{l}0 \\
0\end{array}$ & $\begin{array}{l}286 \\
477 \\
805\end{array}$ & $\begin{array}{r}10 \\
0\end{array}$ & $\begin{array}{r}-94 \\
76\end{array}$ & $\begin{array}{l}45 \\
15\end{array}$ & $\begin{array}{r}-191 \\
-3\end{array}$ & $\begin{array}{r}95 \\
100\end{array}$ & $\begin{array}{r}-486 \\
-318 \\
35\end{array}$ & $\begin{array}{r}10 \\
0\end{array}$ & $\begin{array}{r}-531 \\
-355 \\
-5\end{array}$ & $\begin{array}{r}10 \\
0\end{array}$ & $\begin{array}{l}-611 \\
-446 \\
-62\end{array}$ & $\begin{array}{l}35 \\
56 \\
66\end{array}$ & & & $\begin{array}{l}-646 \\
-502 \\
-128\end{array}$ & $\begin{array}{l}0 \\
0\end{array}$ & & & $\begin{array}{l}4058 \\
3833 \\
1867 \\
3022 \\
2004\end{array}$ & $\begin{array}{l}98 \\
92 \\
88\end{array}$ & $\begin{array}{l}\mathrm{J} \\
\mathrm{J} \\
\mathrm{k} \\
\mathrm{J}\end{array}$ \\
\hline 788 & 40 & & & & & -116 & 13 & -129 & 118 & -448 & 35 & -498 & & $-58 B$ & 19 & -607 & & -640 & & & & 2420 & & K \\
\hline $\begin{array}{l}270 \\
195\end{array}$ & $\begin{array}{l}45 \\
35\end{array}$ & $\begin{array}{r}140 \\
77\end{array}$ & $\begin{array}{l}0 \\
0\end{array}$ & $\begin{array}{r}29 \\
-50\end{array}$ & $\begin{array}{l}0 \\
0\end{array}$ & $\begin{array}{l}-306 \\
-383\end{array}$ & $\begin{array}{l}0 \\
0\end{array}$ & $\begin{array}{l}-393 \\
-462\end{array}$ & $\begin{array}{l}125 \\
126\end{array}$ & $\begin{array}{l}-685 \\
-742\end{array}$ & $\begin{array}{l}35 \\
42\end{array}$ & $\begin{array}{l}-741 \\
-804\end{array}$ & $\begin{array}{r}15 \\
0\end{array}$ & $\begin{array}{l}-836 \\
-937\end{array}$ & $\begin{array}{r}0 \\
10\end{array}$ & & & $\begin{array}{l}-862 \\
-957\end{array}$ & $\begin{array}{r}10 \\
0\end{array}$ & $\begin{array}{l}-1200 \\
-1244\end{array}$ & $\begin{array}{l}175 \\
160\end{array}$ & $\begin{array}{l}4550 \\
8172\end{array}$ & $\begin{array}{l}110 \\
1143\end{array}$ & $\begin{array}{l}T \\
T\end{array}$ \\
\hline $\begin{array}{l}-497 \\
-641\end{array}$ & $\begin{array}{l}15 \\
20\end{array}$ & $\begin{array}{l}-602 \\
-742\end{array}$ & $\begin{array}{l}0 \\
0\end{array}$ & $\begin{array}{l}-755 \\
-900\end{array}$ & $\begin{array}{l}0 \\
0\end{array}$ & $\begin{array}{r}-679 \\
-994 \\
-1109 \\
-1235\end{array}$ & $\begin{array}{r}15 \\
15 \\
0 \\
0\end{array}$ & $\begin{array}{r}-715 \\
-1010 \\
-1120 \\
-1248\end{array}$ & $\begin{array}{r}90 \\
145 \\
128 \\
122\end{array}$ & $\begin{array}{l}-1005 \\
-1315 \\
-1395 \\
-1521\end{array}$ & $\begin{array}{l}45 \\
45 \\
57 \\
59\end{array}$ & $\begin{array}{l}-1150 \\
-1360 \\
-1452 \\
-1580\end{array}$ & $\begin{array}{l}10 \\
10\end{array}$ & $\begin{array}{l}-1159 \\
-1445 \\
-1571 \\
-1686\end{array}$ & $\begin{array}{l}11 \\
51 \\
46\end{array}$ & $\begin{array}{l}-1170 \\
-1468\end{array}$ & $\begin{array}{l}0 \\
0\end{array}$ & $\begin{array}{l}-1170 \\
-1468 \\
-1622 \\
-1732\end{array}$ & $\begin{array}{r}0 \\
15\end{array}$ & $\begin{array}{l}-1170 \\
-2048 \\
-2174\end{array}$ & $\begin{array}{l}160 \\
160\end{array}$ & $\begin{array}{l}8475 \\
8273\end{array}$ & $\begin{array}{l}179 \\
194\end{array}$ & $\begin{array}{l}T \\
T\end{array}$ \\
\hline & & & & & & -1307 & 15 & -1332 & 94 & -1602 & 50 & -1652 & & & & & & & & & & & & \\
\hline $\begin{array}{l}-743 \\
-759\end{array}$ & $\begin{array}{l}40 \\
25\end{array}$ & $\begin{array}{l}-828 \\
-837\end{array}$ & $\begin{array}{l}0 \\
0\end{array}$ & $\begin{array}{l}-989 \\
-978\end{array}$ & $\begin{array}{l}0 \\
0\end{array}$ & $\begin{array}{l}-1302 \\
-1228\end{array}$ & $\begin{array}{l}0 \\
0\end{array}$ & $\begin{array}{l}-1313 \\
-1246\end{array}$ & $\begin{array}{l}99 \\
89\end{array}$ & $\begin{array}{l}-1579 \\
-1553\end{array}$ & $\begin{array}{l}51 \\
39\end{array}$ & $\begin{array}{l}-1630 \\
-1609\end{array}$ & $\begin{array}{l}10 \\
30\end{array}$ & $\begin{array}{l}-1754 \\
-1822\end{array}$ & $\begin{array}{l}40 \\
17\end{array}$ & & & $\begin{array}{l}-1794 \\
-1839\end{array}$ & $\begin{array}{l}30 \\
30\end{array}$ & $\begin{array}{l}-2271 \\
-2321\end{array}$ & $\begin{array}{l}180 \\
210\end{array}$ & $\begin{array}{r}9200 \\
10002\end{array}$ & $\begin{array}{l}194 \\
176\end{array}$ & $\begin{array}{l}4 \\
4 \\
\end{array}$ \\
\hline & & & & & & -1293 & 15 & -1333 & 100 & -1608 & 45 & -1653 & & & & & & & & & & & & \\
\hline $\begin{array}{r}-934 \\
-1011\end{array}$ & $\begin{array}{l}40 \\
40\end{array}$ & $\begin{array}{l}-1009 \\
-1095\end{array}$ & $\begin{array}{l}0 \\
0\end{array}$ & $\begin{array}{l}-1149 \\
-1236\end{array}$ & $\begin{array}{l}0 \\
0\end{array}$ & $\begin{array}{l}-1398 \\
-1451\end{array}$ & $\begin{array}{l}25 \\
40\end{array}$ & $\begin{array}{l}-1423 \\
-1517\end{array}$ & $\begin{array}{r}92 \\
109\end{array}$ & $\begin{array}{l}-1696 \\
-1810\end{array}$ & $\begin{array}{l}50 \\
51\end{array}$ & $\begin{array}{l}-1756 \\
-1874\end{array}$ & $\begin{array}{l}80 \\
45\end{array}$ & $\begin{array}{l}-1986 \\
-2051\end{array}$ & $\begin{array}{l}38 \\
17\end{array}$ & & & $\begin{array}{l}-2026 \\
-2068\end{array}$ & $\begin{array}{l}40 \\
40\end{array}$ & $\begin{array}{l}-2500 \\
-2567\end{array}$ & $\begin{array}{l}230 \\
230\end{array}$ & $\begin{array}{l}8979 \\
9355\end{array}$ & $\begin{array}{l}186 \\
193\end{array}$ & $T$ \\
\hline $\begin{array}{l}-11125 \\
-11151 \\
-1206 \\
-1274 \\
-1433\end{array}$ & $\begin{array}{l}30 \\
40 \\
30 \\
25 \\
20\end{array}$ & $\begin{array}{l}-1213 \\
-1246 \\
-1305 \\
-1375 \\
-1540\end{array}$ & $\begin{array}{l}0 \\
0 \\
0 \\
0 \\
0\end{array}$ & $\begin{array}{l}-1349 \\
-1371 \\
-1445 \\
-1528 \\
-1691\end{array}$ & $\begin{array}{l}0 \\
0 \\
0 \\
0 \\
0\end{array}$ & $\begin{array}{l}-1560 \\
-1585 \\
-1662 \\
-1701 \\
-1858\end{array}$ & $\begin{array}{l}53 \\
25 \\
25 \\
55 \\
56\end{array}$ & $\begin{array}{l}-1613 \\
-1617 \\
-1700 \\
-1766 \\
-1924\end{array}$ & $\begin{array}{r}126 \\
102 \\
112 \\
96 \\
105\end{array}$ & $\begin{array}{l}-1912 \\
-1886 \\
-1960 \\
-2026 \\
-2204\end{array}$ & $\begin{array}{l}58 \\
65 \\
48 \\
54 \\
51\end{array}$ & $\begin{array}{l}-1970 \\
-1951 \\
-2008 \\
-2080 \\
-2255\end{array}$ & $\begin{array}{l}55 \\
30 \\
55 \\
50 \\
45\end{array}$ & $\begin{array}{l}-2147 \\
-2215 \\
-2289 \\
-2379 \\
-2581\end{array}$ & $\begin{array}{r}20 \\
10 \\
10 \\
0 \\
10\end{array}$ & & & $\begin{array}{l}-2167 \\
-2251 \\
-2315 \\
-2407 \\
-2611\end{array}$ & $\begin{array}{l}35 \\
35 \\
20 \\
10 \\
20\end{array}$ & $\begin{array}{l}-2673 \\
-2673 \\
-2729 \\
-2807 \\
-3022\end{array}$ & $\begin{array}{l}190 \\
210 \\
225 \\
240 \\
230\end{array}$ & $\begin{array}{r}10324 \\
6757 \\
7000 \\
10712 \\
6928\end{array}$ & $\begin{array}{l}175 \\
130 \\
142 \\
175 \\
170\end{array}$ & $\begin{array}{l}T \\
T \\
T \\
T \\
T \\
T\end{array}$ \\
\hline $\begin{array}{l}2194 \\
2636 \\
2939\end{array}$ & $\begin{array}{l}13 \\
15 \\
16\end{array}$ & $\begin{array}{l}2164 \\
2604 \\
2908\end{array}$ & $\begin{array}{l}0 \\
0 \\
0\end{array}$ & $\begin{array}{l}2094 \\
2521 \\
2800 \\
3037 \\
3144\end{array}$ & $\begin{array}{l}30 \\
30 \\
25 \\
28 \\
31\end{array}$ & $\begin{array}{l}1951 \\
2397 \\
2684 \\
2929 \\
3032\end{array}$ & $\begin{array}{r}65 \\
60 \\
75 \\
95 \\
120\end{array}$ & $\begin{array}{l}1812 \\
2221 \\
2493 \\
2705 \\
2798\end{array}$ & $\begin{array}{l}60 \\
35 \\
70 \\
60 \\
55\end{array}$ & $\begin{array}{l}1510 \\
1924 \\
2208 \\
2425 \\
2521\end{array}$ & $\begin{array}{l}30 \\
20 \\
20 \\
34 \\
26\end{array}$ & $\begin{array}{l}1473 \\
1893 \\
2177 \\
2391 \\
2495\end{array}$ & $\begin{array}{r}150 \\
149 \\
95 \\
75 \\
90\end{array}$ & $\begin{array}{r}896 \\
1307 \\
1747 \\
2021 \\
2126\end{array}$ & $\begin{array}{l}48 \\
31 \\
45 \\
20 \\
32\end{array}$ & $\begin{array}{l}2091 \\
2094\end{array}$ & $\begin{array}{l}0 \\
0\end{array}$ & $\begin{array}{l}1702 \\
1971 \\
2070\end{array}$ & $\begin{array}{l}40 \\
75 \\
80\end{array}$ & $\begin{array}{l}1269 \\
1659 \\
1871 \\
1971\end{array}$ & $\begin{array}{l}15 \\
15 \\
12 \\
13\end{array}$ & $\begin{array}{l}3201 \\
2863 \\
2502 \\
1670 \\
1540\end{array}$ & $\begin{array}{l}80 \\
92 \\
79\end{array}$ & $\begin{array}{l}J \\
M \\
M \\
M\end{array}$ \\
\hline $\begin{array}{l}3032 \\
2937 \\
2892 \\
2660 \\
2644\end{array}$ & $\begin{array}{l}20 \\
30 \\
25 \\
20 \\
21\end{array}$ & $\begin{array}{l}2979 \\
2884 \\
2838 \\
2590 \\
2569\end{array}$ & $\begin{array}{l}0 \\
0 \\
0 \\
0 \\
0 .\end{array}$ & $\begin{array}{l}2896 \\
2819 \\
2774 \\
2496 \\
2479\end{array}$ & $\begin{array}{l}37 \\
25 \\
25 \\
25 \\
24\end{array}$ & $\begin{array}{l}2766 \\
2663 \\
2013 \\
2331 \\
2305\end{array}$ & $\begin{array}{r}160 \\
130 \\
130 \\
105 \\
80\end{array}$ & $\begin{array}{l}2520 \\
2418 \\
2371 \\
2113 \\
2080\end{array}$ & $\begin{array}{l}75 \\
60 \\
70 \\
55 \\
65\end{array}$ & $\begin{array}{l}2269 \\
2143 \\
2111 \\
1815 \\
1768\end{array}$ & $\begin{array}{l}39 \\
45 \\
45 \\
34 \\
20\end{array}$ & $\begin{array}{l}2230 \\
2098 \\
2006 \\
1781 \\
1739\end{array}$ & $\begin{array}{l}70 \\
60 \\
65 \\
49 \\
55\end{array}$ & $\begin{array}{l}1862 \\
1742 \\
1693 \\
1338 \\
1309\end{array}$ & $\begin{array}{l}23 \\
33 \\
30 \\
21 \\
20\end{array}$ & & & $\begin{array}{l}1829 \\
1693 \\
1651 \\
1317 \\
1289\end{array}$ & $\begin{array}{r}60 \\
77 \\
65 \\
0 \\
0\end{array}$ & $\begin{array}{l}1755 \\
1587 \\
1554 \\
1289 \\
1270\end{array}$ & $\begin{array}{r}10 \\
16 \\
5 \\
15 \\
25\end{array}$ & $\begin{array}{l}2047 \\
2036 \\
2040 \\
2698 \\
2980\end{array}$ & $\begin{array}{l}65 \\
70 \\
75 \\
80 \\
90\end{array}$ & $\begin{array}{c}M \\
M \\
M\end{array}$ \\
\hline $\begin{array}{l}2375 \\
1732 \\
1549 \\
1313 \\
1247\end{array}$ & $\begin{array}{l}16 \\
25 \\
20 \\
20 \\
20\end{array}$ & $\begin{array}{l}2295 \\
1659 \\
1476 \\
1244 \\
1120\end{array}$ & $\begin{array}{l}0 \\
0 \\
0 \\
0 \\
0\end{array}$ & $\begin{array}{l}2210 \\
1556 \\
1374 \\
1149 \\
1071\end{array}$ & $\begin{array}{l}30 \\
28 \\
25 \\
35 \\
35\end{array}$ & $\begin{array}{r}1948 \\
1282 \\
1075 \\
849 \\
769\end{array}$ & $\begin{array}{l}75 \\
75 \\
70 \\
90 \\
95\end{array}$ & $\begin{array}{r}1798 \\
1151 \\
956 \\
704 \\
624\end{array}$ & $\begin{array}{r}75 \\
120 \\
100 \\
65 \\
90\end{array}$ & $\begin{array}{r}1497 \\
822 \\
612 \\
342 \\
279\end{array}$ & $\begin{array}{l}15 \\
15 \\
20 \\
29 \\
20\end{array}$ & $\begin{array}{r}1476 \\
801 \\
591 \\
313 \\
245\end{array}$ & $\begin{array}{r}75 \\
100 \\
25 \\
20 \\
50\end{array}$ & $\begin{array}{r}448 \\
306 \\
31\end{array}$ & $\begin{array}{l}10 \\
32 \\
29\end{array}$ & 2 & 0 & $\begin{array}{r}1094 \\
438 \\
257 \\
-8 \\
-43\end{array}$ & $\begin{array}{l}40 \\
45 \\
33 \\
30 \\
45\end{array}$ & $\begin{array}{r}1017 \\
342 \\
147 \\
-106 \\
-165\end{array}$ & $\begin{array}{l}25 \\
20 \\
50 \\
\\
45\end{array}$ & $\begin{array}{l}3216 \\
3286 \\
3450 \\
3443 \\
3483\end{array}$ & $\begin{array}{l}84 \\
91 \\
83 \\
95 \\
91\end{array}$ & $\begin{array}{l}M \\
M \\
M \\
M\end{array}$ \\
\hline $\begin{array}{l}915 \\
771 \\
643\end{array}$ & $\begin{array}{l}20 \\
15 \\
23\end{array}$ & $\begin{array}{l}855 \\
705 \\
555\end{array}$ & $\begin{array}{l}0 \\
0 \\
0\end{array}$ & $\begin{array}{l}719 \\
559 \\
421\end{array}$ & $\begin{array}{l}42 \\
55 \\
40\end{array}$ & $\begin{array}{r}395 \\
224 \\
72\end{array}$ & $\begin{array}{l}75 \\
75 \\
60\end{array}$ & $\begin{array}{r}217 \\
74 \\
-70\end{array}$ & $\begin{array}{r}85 \\
0 \\
0\end{array}$ & $\begin{array}{r}-46 \\
-195 \\
-354\end{array}$ & $\begin{array}{l}20 \\
25 \\
20\end{array}$ & $\begin{array}{r}-73 \\
-250 \\
-387\end{array}$ & $\begin{array}{l}88 \\
60 \\
55\end{array}$ & $\begin{array}{l}-513 \\
-638\end{array}$ & $\begin{array}{l}30 \\
23\end{array}$ & & & $\begin{array}{l}-372 \\
-545 \\
-695\end{array}$ & $\begin{array}{l}25 \\
15 \\
15\end{array}$ & $\begin{array}{l}-489 \\
-657 \\
-792\end{array}$ & $\begin{array}{l}45 \\
75 \\
75\end{array}$ & $\begin{array}{l}3724 \\
3793 \\
3848\end{array}$ & $\begin{array}{l}94 \\
93 \\
87\end{array}$ & $\begin{array}{c}M \\
M\end{array}$ \\
\hline 602 & 20 & 527 & 0 & 371 & 28 & 30 & 45 & -112 & 0 & -401 & 20 & -428 & 40 & -662 & 32 & & & -694 & 15 & -831 & 50 & 4160 & 95 & $M$ \\
\hline $\begin{array}{l}345 \\
230 \\
225\end{array}$ & $\begin{array}{l}20 \\
15 \\
15\end{array}$ & $\begin{array}{l}284 \\
164 \\
152\end{array}$ & $\begin{array}{l}0 \\
0 \\
0\end{array}$ & $\begin{array}{r}72 \\
-33 \\
-47\end{array}$ & $\begin{array}{l}40 \\
10 \\
10\end{array}$ & $\begin{array}{l}-265 \\
-377 \\
-388\end{array}$ & $\begin{array}{l}58 \\
30 \\
35\end{array}$ & $\begin{array}{l}-390 \\
-499 \\
-513\end{array}$ & $\begin{array}{l}0 \\
0 \\
0\end{array}$ & $\begin{array}{l}-685 \\
-824 \\
-818\end{array}$ & $\begin{array}{l}38 \\
38 \\
25\end{array}$ & $\begin{array}{l}-736 \\
-862 \\
-848\end{array}$ & $\begin{array}{l}55 \\
45 \\
10\end{array}$ & $\begin{array}{l}-1050 \\
-1028\end{array}$ & $\begin{array}{l}10 \\
50\end{array}$ & & & $\begin{array}{r}-938 \\
-1060 \\
-1078\end{array}$ & $\begin{array}{l}25 \\
30 \\
30\end{array}$ & -1087 & 50 & $\begin{array}{l}1990 \\
1496 \\
4133 \\
4130 \\
4106\end{array}$ & $\begin{array}{r}78 \\
71 \\
103 \\
114 \\
107\end{array}$ & $\begin{array}{l}K \\
K \\
M \\
J \\
J\end{array}$ \\
\hline $\begin{array}{l}253 \\
165\end{array}$ & $\begin{array}{l}20 \\
20\end{array}$ & $\begin{array}{r}173 \\
79\end{array}$ & $\begin{array}{l}0 \\
0\end{array}$ & $\begin{array}{r}-20 \\
-115\end{array}$ & $\begin{array}{l}0 \\
0\end{array}$ & $\begin{array}{l}-378 \\
-455\end{array}$ & $\begin{array}{l}65 \\
55\end{array}$ & $\begin{array}{l}-483 \\
-569\end{array}$ & $\begin{array}{l}53 \\
53\end{array}$ & $\begin{array}{l}-784 \\
-875\end{array}$ & $\begin{array}{l}20 \\
20\end{array}$ & $\begin{array}{l}-822 \\
-915\end{array}$ & $\begin{array}{l}0 \\
0\end{array}$ & $\begin{array}{l}-1020 \\
-1081\end{array}$ & $\begin{array}{l}36 \\
19\end{array}$ & & & $\begin{array}{l}-1056 \\
-1100\end{array}$ & $\begin{array}{l}15 \\
45\end{array}$ & & & $\begin{array}{l}4124 \\
4262 \\
1994\end{array}$ & $\begin{array}{r}94 \\
128 \\
73\end{array}$ & $\begin{array}{l}\mathrm{J} \\
\mathrm{J} \\
\mathrm{K}\end{array}$ \\
\hline $\begin{array}{r}84 \\
354\end{array}$ & $\begin{array}{l}30 \\
25\end{array}$ & $\begin{array}{r}4 \\
260\end{array}$ & $\begin{array}{l}0 \\
0\end{array}$ & 72 & 0 & -315 & 25 & -370 & 86 & -717 & 15 & -780 & 0 & -899 & 24 & & & -923 & 20 & & & $\begin{array}{l}3646 \\
4447\end{array}$ & $\begin{array}{r}70 \\
105\end{array}$ & $\begin{array}{l}\mathrm{k} \\
\mathrm{J}\end{array}$ \\
\hline $\begin{array}{l}662 \\
785\end{array}$ & $\begin{array}{l}10 \\
10\end{array}$ & $\begin{array}{l}556 \\
717\end{array}$ & $\begin{array}{l}0 \\
0\end{array}$ & $\begin{array}{l}358 \\
502\end{array}$ & $\begin{array}{l}0 \\
0\end{array}$ & $\begin{array}{r}-154 \\
-37 \\
111 \\
181 \\
250\end{array}$ & $\begin{array}{l}30 \\
15 \\
20 \\
40 \\
20\end{array}$ & $\begin{array}{r}-195 \\
-86 \\
61 \\
131\end{array}$ & $\begin{array}{r}90 \\
92 \\
103 \\
102\end{array}$ & $\begin{array}{l}-530 \\
-417 \\
-291 \\
-219\end{array}$ & $\begin{array}{l}20 \\
15 \\
15 \\
20\end{array}$ & $\begin{array}{l}-588 \\
-468 \\
-344 \\
-257\end{array}$ & $\begin{array}{r}30 \\
0\end{array}$ & $\begin{array}{l}-592 \\
-461\end{array}$ & $\begin{array}{l}46 \\
20\end{array}$ & & & $\begin{array}{l}-638 \\
-488\end{array}$ & $\begin{array}{l}0 \\
0\end{array}$ & -884 & 85 & $\begin{array}{l}4461 \\
3782\end{array}$ & $\begin{array}{l}98 \\
90\end{array}$ & $\mathrm{M}$ \\
\hline
\end{tabular}




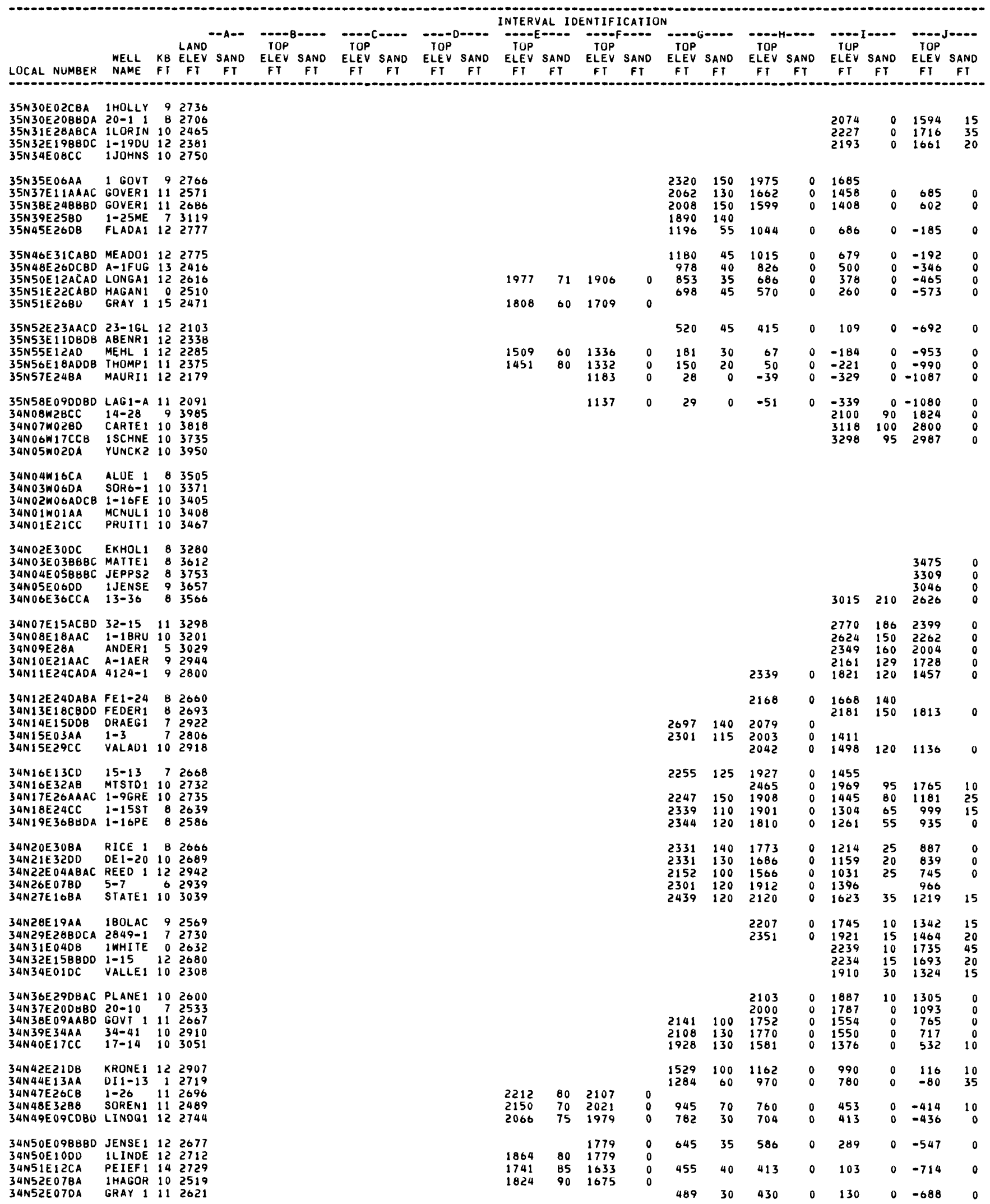




\begin{tabular}{|c|c|c|c|c|c|c|c|c|c|c|c|c|c|c|c|c|c|c|c|c|c|c|c|c|}
\hline $\begin{array}{l}1044 \\
1184 \\
1133\end{array}$ & $\begin{array}{r}10 \\
0 \\
10\end{array}$ & $\begin{array}{r}947 \\
1087\end{array}$ & $\begin{array}{l}0 \\
0\end{array}$ & $\begin{array}{l}734 \\
857\end{array}$ & $\begin{array}{l}0 \\
0\end{array}$ & $\begin{array}{l}361 \\
334 \\
441\end{array}$ & $\begin{array}{l}30 \\
15 \\
45\end{array}$ & $\begin{array}{l}317 \\
385\end{array}$ & 105 & 60 & $\begin{array}{l}20 \\
20\end{array}$ & $\begin{array}{r}-40 \\
15\end{array}$ & 40 & -92 & 50 & -166 & 0 & & & & & $\begin{array}{l}2492 \\
2661 \\
1551\end{array}$ & 82 & $\begin{array}{l}k \\
j \\
k\end{array}$ \\
\hline & & & & & & & & 56 & 114 & -225 & 30 & -275 & & & & & & & & & & & & \\
\hline $\begin{array}{l}283 \\
212\end{array}$ & $\begin{array}{l}0 \\
0\end{array}$ & $\begin{array}{l}198 \\
114\end{array}$ & $\begin{array}{l}0 \\
0\end{array}$ & $\begin{array}{r}51 \\
-35\end{array}$ & $\begin{array}{l}0 \\
0\end{array}$ & $\begin{array}{l}-144 \\
-332 \\
-400\end{array}$ & $\begin{array}{r}30 \\
0 \\
0\end{array}$ & $\begin{array}{l}-174 \\
-395 \\
-465\end{array}$ & $\begin{array}{l}179 \\
121 \\
127\end{array}$ & $\begin{array}{l}-468 \\
-679 \\
-763\end{array}$ & $\begin{array}{l}40 \\
40 \\
60\end{array}$ & $\begin{array}{l}-524 \\
-735 \\
-823\end{array}$ & $\begin{array}{l}15 \\
25\end{array}$ & $\begin{array}{l}-888 \\
-934\end{array}$ & $\begin{array}{l}20 \\
25\end{array}$ & & & $\begin{array}{l}-908 \\
-977\end{array}$ & $\begin{array}{l}0 \\
0\end{array}$ & $\begin{array}{l}-1224 \\
-1310\end{array}$ & $\begin{array}{l}135 \\
105\end{array}$ & $\begin{array}{l}4848 \\
5127 \\
1694\end{array}$ & $\begin{array}{r}113 \\
115 \\
70\end{array}$ & $\begin{array}{l}M \\
M \\
K\end{array}$ \\
\hline-534 & 10 & -659 & 0 & -818 & 0 & -1160 & 0 & -1171 & 52 & -1441 & 49 & -1490 & 10 & -1614 & 41 & & & -1655 & 10 & -2137 & 150 & 7396 & 194 & $T$ \\
\hline $\begin{array}{l}-545 \\
-700 \\
-814 \\
-920\end{array}$ & $\begin{array}{l}10 \\
10 \\
20 \\
20\end{array}$ & $\begin{array}{r}-667 \\
-820 \\
-902 \\
-1024\end{array}$ & $\begin{array}{l}0 \\
0 \\
0 \\
0\end{array}$ & $\begin{array}{r}-833 \\
-974 \\
-1052 \\
-1182\end{array}$ & $\begin{array}{l}0 \\
0 \\
0 \\
0\end{array}$ & $\begin{array}{l}-1170 \\
-1293 \\
-1342 \\
-1460\end{array}$ & $\begin{array}{l}0 \\
0 \\
0 \\
0\end{array}$ & $\begin{array}{l}-1180 \\
-1305 \\
-1352 \\
-1470\end{array}$ & $\begin{array}{r}115 \\
98 \\
87 \\
81\end{array}$ & $\begin{array}{l}-1436 \\
-1589 \\
-1632 \\
-1721\end{array}$ & $\begin{array}{l}52 \\
59 \\
30 \\
60\end{array}$ & $\begin{array}{l}-1488 \\
-1648 \\
-1692 \\
-1787\end{array}$ & $\begin{array}{r}0 \\
20 \\
75 \\
70\end{array}$ & $\begin{array}{l}-1605 \\
-1735 \\
-1890 \\
-2020\end{array}$ & $\begin{array}{l}25 \\
84 \\
40 \\
24\end{array}$ & & & $\begin{array}{l}-1653 \\
-1821 \\
-1930 \\
-2044\end{array}$ & $\begin{array}{l}10 \\
15 \\
15 \\
15\end{array}$ & $\begin{array}{l}-2151 \\
-2331 \\
-2399 \\
-2523\end{array}$ & $\begin{array}{l}145 \\
145 \\
145 \\
135\end{array}$ & $\begin{array}{r}8415 \\
8310 \\
9964 \\
10006\end{array}$ & $\begin{array}{l}172 \\
137 \\
190 \\
194\end{array}$ & $\begin{array}{l}\mathbf{T} \\
\mathbf{T} \\
\mathbf{T} \\
\mathbf{T}\end{array}$ \\
\hline $\begin{array}{l}-1052 \\
-1314 \\
-1347 \\
-1466\end{array}$ & $\begin{array}{l}15 \\
15 \\
20 \\
15\end{array}$ & $\begin{array}{l}-1148 \\
-1430 \\
-1462 \\
-1569\end{array}$ & $\begin{array}{l}0 \\
0 \\
0 \\
0\end{array}$ & $\begin{array}{l}-1292 \\
-1350 \\
-1577 \\
-1608 \\
-1732\end{array}$ & $\begin{array}{l}0 \\
0 \\
0 \\
0 \\
0\end{array}$ & $\begin{array}{l}-1575 \\
-1564 \\
-1778 \\
-1808 \\
-1922\end{array}$ & $\begin{array}{l}0 \\
50 \\
20 \\
35 \\
30\end{array}$ & $\begin{array}{l}-1578 \\
-1625 \\
-1826 \\
-1851 \\
-1966\end{array}$ & $\begin{array}{r}101 \\
98 \\
127 \\
118 \\
97\end{array}$ & $\begin{array}{l}-1849 \\
-1901 \\
-2112 \\
-2132 \\
-2233\end{array}$ & $\begin{array}{l}59 \\
51 \\
51 \\
51 \\
70\end{array}$ & $\begin{array}{l}-1908 \\
-1952 \\
-2163 \\
-2183 \\
-2303\end{array}$ & $\begin{array}{l}95 \\
85 \\
80 \\
95 \\
25\end{array}$ & $\begin{array}{l}-2139 \\
-2218 \\
-2470 \\
-2524 \\
-2633\end{array}$ & $\begin{array}{l}43 \\
42 \\
10 \\
10 \\
36\end{array}$ & & & $\begin{array}{l}-2182 \\
-2260 \\
-2498 \\
-2553 \\
-2669\end{array}$ & $\begin{array}{l}30 \\
35 \\
25 \\
10 \\
15\end{array}$ & $\begin{array}{l}-2659 \\
-2731 \\
-2910 \\
-2964 \\
-3081\end{array}$ & $\begin{array}{l}140 \\
200 \\
255 \\
235 \\
215\end{array}$ & $\begin{array}{r}10004 \\
9546 \\
7004 \\
7192 \\
7387\end{array}$ & $\begin{array}{l}175 \\
178 \\
136 \\
145 \\
141\end{array}$ & $\begin{array}{l}\mathrm{T} \\
\mathrm{T} \\
\mathrm{T} \\
\mathrm{T} \\
\mathrm{T}\end{array}$ \\
\hline $\begin{array}{r}-1436 \\
856 \\
1813 \\
2001 \\
2758\end{array}$ & $\begin{array}{l}25 \\
30 \\
15 \\
20 \\
16\end{array}$ & $\begin{array}{r}-1531 \\
764 \\
1769 \\
1951 \\
2718\end{array}$ & $\begin{array}{l}0 \\
0 \\
0 \\
0 \\
0\end{array}$ & $\begin{array}{r}-1695 \\
707 \\
1720 \\
1898 \\
2636\end{array}$ & $\begin{array}{r}0 \\
34 \\
20 \\
30 \\
20\end{array}$ & $\begin{array}{r}-1875 \\
593 \\
1583 \\
1723 \\
2469\end{array}$ & $\begin{array}{l}40 \\
40 \\
45 \\
35 \\
26\end{array}$ & $\begin{array}{r}-1934 \\
453 \\
1439 \\
1595 \\
2347\end{array}$ & $\begin{array}{l}90 \\
65 \\
75 \\
95 \\
55\end{array}$ & $\begin{array}{r}-2170 \\
92 \\
1112 \\
1276 \\
2037\end{array}$ & $\begin{array}{l}60 \\
30 \\
40 \\
41 \\
35\end{array}$ & $\begin{array}{r}-2238 \\
44 \\
1070 \\
1235 \\
2002\end{array}$ & $\begin{array}{l}45 \\
235 \\
160 \\
120 \\
110\end{array}$ & $\begin{array}{r}-2561 \\
-568 \\
460 \\
652 \\
1488\end{array}$ & $\begin{array}{r}32 \\
110 \\
74 \\
70 \\
55\end{array}$ & & & $\begin{array}{r}-2593 \\
-718 \\
386\end{array}$ & $\begin{array}{r}15 \\
0 \\
0\end{array}$ & $\begin{array}{r}-2998 \\
-755 \\
363 \\
576 \\
1420\end{array}$ & $\begin{array}{r}230 \\
30 \\
40 \\
45 \\
30\end{array}$ & $\begin{array}{l}7336 \\
7503 \\
3704 \\
3436 \\
2797\end{array}$ & $\begin{array}{r}141 \\
127 \\
85 \\
85 \\
86\end{array}$ & $\begin{array}{l}T \\
M \\
M \\
M \\
M\end{array}$ \\
\hline $\begin{array}{l}2870 \\
3025\end{array}$ & $\begin{array}{l}24 \\
15\end{array}$ & $\begin{array}{l}2825 \\
2990\end{array}$ & $\begin{array}{l}0 \\
0\end{array}$ & $\begin{array}{l}2741 \\
2945 \\
\\
3167 \\
3069\end{array}$ & $\begin{array}{l}40 \\
30 \\
40 \\
35\end{array}$ & $\begin{array}{l}2618 \\
2819 \\
3073 \\
3028 \\
2940\end{array}$ & $\begin{array}{r}105 \\
80 \\
80 \\
55 \\
65\end{array}$ & $\begin{array}{l}2423 \\
2623 \\
2857 \\
2864 \\
2761\end{array}$ & $\begin{array}{r}95 \\
75 \\
40 \\
95 \\
120\end{array}$ & $\begin{array}{l}2144 \\
2344 \\
2570 \\
2536 \\
2414\end{array}$ & $\begin{array}{l}36 \\
25 \\
26 \\
20 \\
24\end{array}$ & $\begin{array}{l}2093 \\
2309 \\
2544 \\
2506 \\
2382\end{array}$ & $\begin{array}{r}90 \\
110 \\
75 \\
65 \\
80\end{array}$ & $\begin{array}{l}1660 \\
1933 \\
2156 \\
2162 \\
2018\end{array}$ & $\begin{array}{l}41 \\
17 \\
42 \\
15 \\
39\end{array}$ & $\begin{array}{l}1899 \\
2114 \\
2147 \\
1979\end{array}$ & $\begin{array}{l}0 \\
0 \\
0 \\
0\end{array}$ & $\begin{array}{l}1619 \\
1864 \\
2093 \\
2118 \\
1970\end{array}$ & $\begin{array}{l}64 \\
75 \\
70 \\
90 \\
85\end{array}$ & $\begin{array}{l}1527 \\
1771 \\
1998 \\
1860\end{array}$ & $\begin{array}{l}12 \\
18 \\
10\end{array}$ & $\begin{array}{l}2162 \\
1805 \\
1589 \\
1444 \\
1775\end{array}$ & $\begin{array}{l}78 \\
74 \\
67 \\
70\end{array}$ & $\begin{array}{l}M \\
M \\
M \\
J \\
M\end{array}$ \\
\hline $\begin{array}{l}3010 \\
2660 \\
2538 \\
2270 \\
1821\end{array}$ & $\begin{array}{l}15 \\
15 \\
20 \\
15 \\
15\end{array}$ & $\begin{array}{l}2958 \\
2624 \\
2494 \\
2216 \\
1772\end{array}$ & $\begin{array}{l}0 \\
0 \\
0 \\
0 \\
0\end{array}$ & $\begin{array}{l}2890 \\
2525 \\
2373 \\
2116 \\
1666\end{array}$ & $\begin{array}{l}35 \\
50 \\
40 \\
35 \\
40\end{array}$ & $\begin{array}{l}2736 \\
2320 \\
2188 \\
1912 \\
1429\end{array}$ & $\begin{array}{l}80 \\
80 \\
70 \\
90 \\
75\end{array}$ & $\begin{array}{l}2558 \\
2142 \\
2008 \\
1734 \\
1270\end{array}$ & $\begin{array}{r}35 \\
75 \\
80 \\
105 \\
75\end{array}$ & $\begin{array}{l}2234 \\
1808 \\
1678 \\
1400 \\
934\end{array}$ & $\begin{array}{l}30 \\
32 \\
22 \\
16 \\
15\end{array}$ & $\begin{array}{r}2156 \\
1772 \\
1649 \\
1376 \\
913\end{array}$ & $\begin{array}{r}25 \\
60 \\
122 \\
75 \\
35\end{array}$ & $\begin{array}{l}1795 \\
1191 \\
559\end{array}$ & $\begin{array}{l}17 \\
15\end{array}$ & 1778 & 0 & $\begin{array}{r}1737 \\
1348 \\
1151 \\
948 \\
519\end{array}$ & $\begin{array}{r}60 \\
38 \\
0 \\
14 \\
40\end{array}$ & $\begin{array}{r}1663 \\
1135 \\
889 \\
443\end{array}$ & $\begin{array}{l}10 \\
15 \\
16\end{array}$ & $\begin{array}{l}1792 \\
2373 \\
2809 \\
2866 \\
3355\end{array}$ & $\begin{array}{l}73 \\
78 \\
92 \\
82 \\
97\end{array}$ & $\begin{array}{l}M \\
J \\
M \\
M \\
M\end{array}$ \\
\hline $\begin{array}{r}1623 \\
1479 \\
1234 \\
1043 \\
775\end{array}$ & $\begin{array}{l}20 \\
22 \\
22 \\
17 \\
20\end{array}$ & $\begin{array}{r}1564 \\
1424 \\
1177 \\
994 \\
708\end{array}$ & $\begin{array}{l}0 \\
0 \\
0 \\
0 \\
0\end{array}$ & $\begin{array}{r}1509 \\
1331 \\
1079 \\
853 \\
559\end{array}$ & $\begin{array}{l}45 \\
60 \\
45 \\
40 \\
50\end{array}$ & $\begin{array}{r}1218 \\
1047 \\
781 \\
513 \\
235\end{array}$ & $\begin{array}{l}70 \\
85 \\
65 \\
75 \\
95\end{array}$ & $\begin{array}{r}1041 \\
919 \\
640 \\
384 \\
85\end{array}$ & $\begin{array}{l}55 \\
65 \\
85 \\
90 \\
75\end{array}$ & $\begin{array}{r}727 \\
586 \\
297 \\
75 \\
-221\end{array}$ & $\begin{array}{r}15 \\
20 \\
20 \\
18 \\
6\end{array}$ & $\begin{array}{r}701 \\
556 \\
266 \\
44 \\
-247\end{array}$ & $\begin{array}{r}25 \\
19 \\
115 \\
30 \\
30\end{array}$ & $\begin{array}{r}418 \\
271 \\
-17 \\
-216 \\
-492\end{array}$ & $\begin{array}{l}47 \\
25 \\
17 \\
24 \\
11\end{array}$ & $\begin{array}{r}371 \\
246 \\
-34 \\
-240 \\
-503\end{array}$ & $\begin{array}{l}0 \\
0 \\
0 \\
0 \\
0\end{array}$ & $\begin{array}{r}355 \\
227 \\
-48 \\
-284 \\
-536\end{array}$ & $\begin{array}{l}53 \\
75 \\
30 \\
33 \\
50\end{array}$ & $\begin{array}{r}244 \\
124 \\
-166 \\
-412 \\
-671\end{array}$ & $\begin{array}{l}25 \\
30 \\
70 \\
50 \\
60\end{array}$ & $\begin{array}{l}3242 \\
3374 \\
3580 \\
3633 \\
3801\end{array}$ & $\begin{array}{r}86 \\
84 \\
108 \\
94\end{array}$ & $\begin{array}{l}M \\
M \\
M \\
M \\
M\end{array}$ \\
\hline 1690 & 125 & & & & & & & & & & & & & & & & & & & & & $\begin{array}{l}1297 \\
1332 \\
1495\end{array}$ & $\begin{array}{l}71 \\
72\end{array}$ & $\begin{array}{l}k \\
k \\
k\end{array}$ \\
\hline 501 & 25 & 428 & 0 & 248 & 53 & -108 & 60 & -235 & 28 & -536 & 20 & -571 & 20 & -774 & 12 & -786 & 0 & -810 & 24 & -957 & 55 & 4277 & 94 & M \\
\hline $\begin{array}{l}448 \\
415 \\
306 \\
240\end{array}$ & $\begin{array}{l}25 \\
25 \\
25 \\
15\end{array}$ & $\begin{array}{l}371 \\
325 \\
210 \\
170\end{array}$ & $\begin{array}{l}0 \\
0 \\
0 \\
0\end{array}$ & $\begin{array}{r}185 \\
137 \\
19 \\
-29\end{array}$ & $\begin{array}{r}10 \\
0 \\
0 \\
0\end{array}$ & $\begin{array}{l}-168 \\
-231 \\
-353 \\
-387\end{array}$ & $\begin{array}{l}30 \\
25 \\
35 \\
35\end{array}$ & $\begin{array}{l}-287 \\
-333 \\
-457 \\
-482\end{array}$ & $\begin{array}{l}60 \\
47 \\
53 \\
49\end{array}$ & $\begin{array}{l}-566 \\
-643 \\
-767 \\
-776\end{array}$ & $\begin{array}{l}10 \\
10 \\
20 \\
10\end{array}$ & $\begin{array}{l}-588 \\
-667 \\
-793 \\
-799\end{array}$ & $\begin{array}{r}10 \\
0 \\
0 \\
60\end{array}$ & $\begin{array}{r}-839 \\
-875 \\
-1024 \\
-1001\end{array}$ & $\begin{array}{r}0 \\
23 \\
15 \\
13\end{array}$ & $\begin{array}{r}-839 \\
-898 \\
-1051 \\
-1014\end{array}$ & $\begin{array}{l}0 \\
0 \\
0 \\
0\end{array}$ & $\begin{array}{r}-839 \\
-898 \\
-1051 \\
-1014\end{array}$ & $\begin{array}{l}30 \\
35 \\
40 \\
40\end{array}$ & -973 & 85 & $\begin{array}{l}4233 \\
4142 \\
4183 \\
4157\end{array}$ & $\begin{array}{l}109 \\
104 \\
105 \\
114\end{array}$ & $\begin{array}{l}\mathrm{M} \\
\mathrm{J} \\
\mathrm{J} \\
\mathrm{J}\end{array}$ \\
\hline $\begin{array}{r}175 \\
157 \\
44\end{array}$ & $\begin{array}{l}15 \\
15 \\
15\end{array}$ & $\begin{array}{r}93 \\
89 \\
-13\end{array}$ & $\begin{array}{l}0 \\
0 \\
0\end{array}$ & $\begin{array}{r}-124 \\
-89 \\
-204\end{array}$ & $\begin{array}{l}0 \\
0 \\
0\end{array}$ & $\begin{array}{l}-528 \\
-455 \\
-567\end{array}$ & $\begin{array}{l}35 \\
20 \\
30\end{array}$ & $\begin{array}{l}-636 \\
-544 \\
-662\end{array}$ & $\begin{array}{l}56 \\
58 \\
67\end{array}$ & $\begin{array}{l}-980 \\
-847 \\
-974\end{array}$ & $\begin{array}{l}15 \\
25 \\
10\end{array}$ & $\begin{array}{r}-1007 \\
-897 \\
-996\end{array}$ & $\begin{array}{r}30 \\
0 \\
20\end{array}$ & $\begin{array}{l}-1244 \\
-1091 \\
-1134\end{array}$ & $\begin{array}{r}25 \\
0 \\
23\end{array}$ & $\begin{array}{l}-1269 \\
-1091 \\
-1157\end{array}$ & $\begin{array}{l}0 \\
0 \\
5\end{array}$ & $\begin{array}{l}-1269 \\
-1091 \\
-1183\end{array}$ & $\begin{array}{l}45 \\
30 \\
80\end{array}$ & & 125 & $\begin{array}{l}4454 \\
4300 \\
5065\end{array}$ & $\begin{array}{l}120 \\
101 \\
112\end{array}$ & $\begin{array}{l}J \\
J \\
M\end{array}$ \\
\hline 632 & 10 & 545 & 0 & 317 & 0 & -89 & 25 & -155 & 124 & -501 & 52 & -553 & 0 & -647 & 44 & -691 & 0 & -738 & 0 & -934 & 90 & 4487 & 101 & M \\
\hline $\begin{array}{r}765 \\
927 \\
1202 \\
1171 \\
812\end{array}$ & $\begin{array}{l}10 \\
10 \\
10 \\
10 \\
10\end{array}$ & 1111 & 0 & 438 & 0 & 452 & $\begin{array}{l}20 \\
20 \\
15\end{array}$ & $\begin{array}{r}-42 \\
338\end{array}$ & $\begin{array}{l}129 \\
111\end{array}$ & $\begin{array}{r}-391 \\
76\end{array}$ & $\begin{array}{l}10 \\
10 \\
30\end{array}$ & -447 & 0 & -578 & 10 & -608 & 0 & -627 & 0 & & & $\begin{array}{l}3707 \\
1908 \\
2747 \\
1733 \\
3191\end{array}$ & $\begin{array}{r}97 \\
84 \\
69 \\
105\end{array}$ & $\begin{array}{l}J \\
k \\
k \\
k \\
J\end{array}$ \\
\hline $\begin{array}{l}784 \\
666 \\
382 \\
380 \\
197\end{array}$ & $\begin{array}{l}10 \\
10 \\
15 \\
25 \\
25\end{array}$ & $\begin{array}{l}684 \\
579 \\
331 \\
317 \\
147\end{array}$ & $\begin{array}{l}0 \\
0 \\
0 \\
0 \\
0\end{array}$ & $\begin{array}{r}457 \\
366 \\
98 \\
87 \\
-71\end{array}$ & $\begin{array}{l}0 \\
0 \\
0 \\
0 \\
0\end{array}$ & $\begin{array}{r}44 \\
-50 \\
-276 \\
-295 \\
-477\end{array}$ & $\begin{array}{r}10 \\
0 \\
0 \\
0 \\
0\end{array}$ & $\begin{array}{r}1 \\
-113 \\
-340 \\
-337 \\
-490\end{array}$ & $\begin{array}{l}113 \\
127 \\
129 \\
115 \\
119\end{array}$ & $\begin{array}{l}-290 \\
-389 \\
-636 \\
-598 \\
-756\end{array}$ & $\begin{array}{l}35 \\
50 \\
35 \\
48 \\
35\end{array}$ & $\begin{array}{l}-345 \\
-450 \\
-693 \\
-646 \\
-808\end{array}$ & $\begin{array}{r}0 \\
10 \\
0 \\
30 \\
80\end{array}$ & $\begin{array}{l}-484 \\
-612 \\
-794 \\
-766 \\
-918\end{array}$ & $\begin{array}{l}22 \\
10 \\
20 \\
15 \\
10\end{array}$ & -506 & 0 & $\begin{array}{l}-520 \\
-638 \\
-832 \\
-800 \\
-932\end{array}$ & $\begin{array}{l}0 \\
0 \\
0 \\
0 \\
0\end{array}$ & $\begin{array}{r}-898 \\
-1155 \\
-1187 \\
-1329\end{array}$ & $\begin{array}{l}130 \\
150 \\
155 \\
175\end{array}$ & $\begin{array}{l}3551 \\
4354 \\
5135 \\
4958 \\
5241\end{array}$ & $\begin{array}{r}92 \\
97 \\
113 \\
130 \\
115\end{array}$ & $\begin{array}{l}J \\
M \\
M \\
M \\
M\end{array}$ \\
\hline $\begin{array}{l}-194 \\
-424\end{array}$ & $\begin{array}{l}10 \\
15\end{array}$ & $\begin{array}{l}-279 \\
-549\end{array}$ & $\begin{array}{l}0 \\
0\end{array}$ & $\begin{array}{l}-477 \\
-720\end{array}$ & $\begin{array}{l}0 \\
0\end{array}$ & $\begin{array}{r}-845 \\
-1013\end{array}$ & $\begin{array}{l}0 \\
0\end{array}$ & $\begin{array}{r}-859 \\
-1060\end{array}$ & $\begin{array}{l}125 \\
119\end{array}$ & $\begin{array}{l}-1145 \\
-1347\end{array}$ & $\begin{array}{l}69 \\
64\end{array}$ & $\begin{array}{l}-1214 \\
-1411\end{array}$ & $\begin{array}{l}10 \\
10\end{array}$ & $\begin{array}{l}-1333 \\
-1520\end{array}$ & $\begin{array}{r}8 \\
29\end{array}$ & -1341 & 0 & $\begin{array}{l}-1360 \\
-1550\end{array}$ & $\begin{array}{r}0 \\
35\end{array}$ & $\begin{array}{l}-1717 \\
-1930\end{array}$ & $\begin{array}{l}175 \\
210\end{array}$ & $\begin{array}{l}7333 \\
7157\end{array}$ & $\begin{array}{l}134 \\
160\end{array}$ & $\begin{array}{l}\mathrm{T} \\
\mathrm{T}\end{array}$ \\
\hline $\begin{array}{l}-755 \\
-767\end{array}$ & $\begin{array}{l}25 \\
15\end{array}$ & $\begin{array}{l}-878 \\
-894\end{array}$ & $\begin{array}{l}0 \\
0\end{array}$ & $\begin{array}{l}-1040 \\
-1052\end{array}$ & $\begin{array}{l}0 \\
0\end{array}$ & $\begin{array}{l}-1309 \\
-1306\end{array}$ & $\begin{array}{l}0 \\
0\end{array}$ & $\begin{array}{l}-1377 \\
-1361\end{array}$ & $\begin{array}{l}93 \\
85\end{array}$ & $\begin{array}{l}-1646 \\
-1631\end{array}$ & $\begin{array}{l}65 \\
30\end{array}$ & $\begin{array}{l}-1711 \\
-1697\end{array}$ & $\begin{array}{l}35 \\
65\end{array}$ & $\begin{array}{l}-1845 \\
-1924\end{array}$ & $\begin{array}{l}10 \\
24\end{array}$ & -1855 & 0 & $\begin{array}{l}-1867 \\
-1948\end{array}$ & $\begin{array}{l}10 \\
10\end{array}$ & $\begin{array}{l}-2402 \\
-2401\end{array}$ & $\begin{array}{r}125 \\
75\end{array}$ & $\begin{array}{l}6389 \\
8217\end{array}$ & $\begin{array}{l}142 \\
165\end{array}$ & $\begin{array}{l}T \\
T\end{array}$ \\
\hline-882 & 30 & -1004 & 0 & -1151 & 0 & -1389 & 0 & -1438 & 78 & -1690 & 69 & -1759 & 85 & -2031 & 23 & & & -2054 & 20 & -2505 & 95 & 7621 & 160 & $T$ \\
\hline-1051 & 35 & -1174 & 0 & -1317 & 0 & -1557 & 0 & -1625 & 95 & -1881 & 34 & -1935 & 60 & -2207 & 29 & & & -2236 & 10 & -2722 & 140 & 9798 & 184 & $\mathrm{~T}$ \\
\hline 1036 & 40 & -1162 & 0 & -1302 & 0 & -1534 & 0 & -1601 & 78 & -1845 & 30 & -1888 & 55 & -2162 & 15 & & & -2188 & 10 & -2681 & 180 & 9666 & 186 & $T$ \\
\hline
\end{tabular}


table 2. - SElectev geologic data -- CONTINUEU

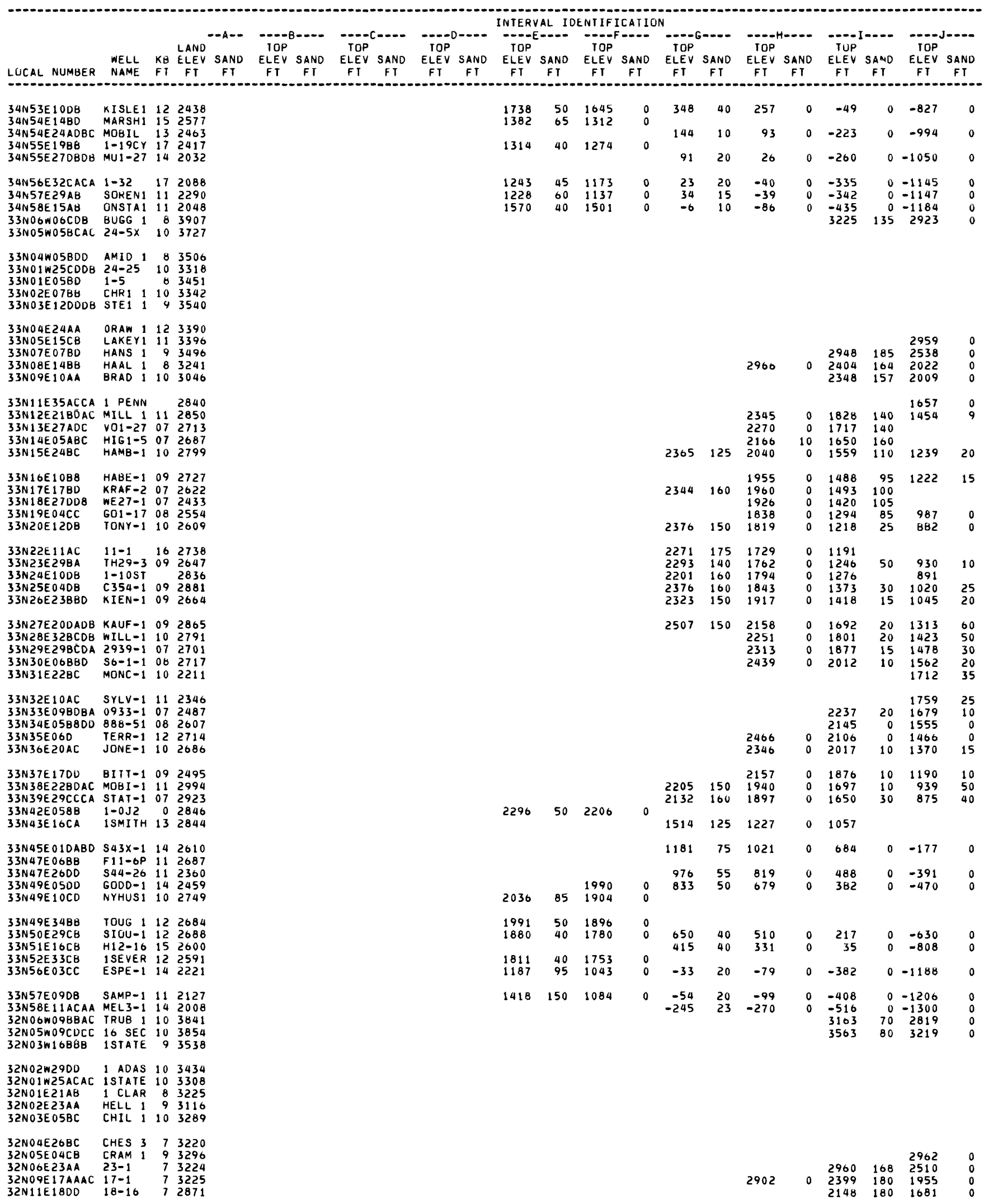




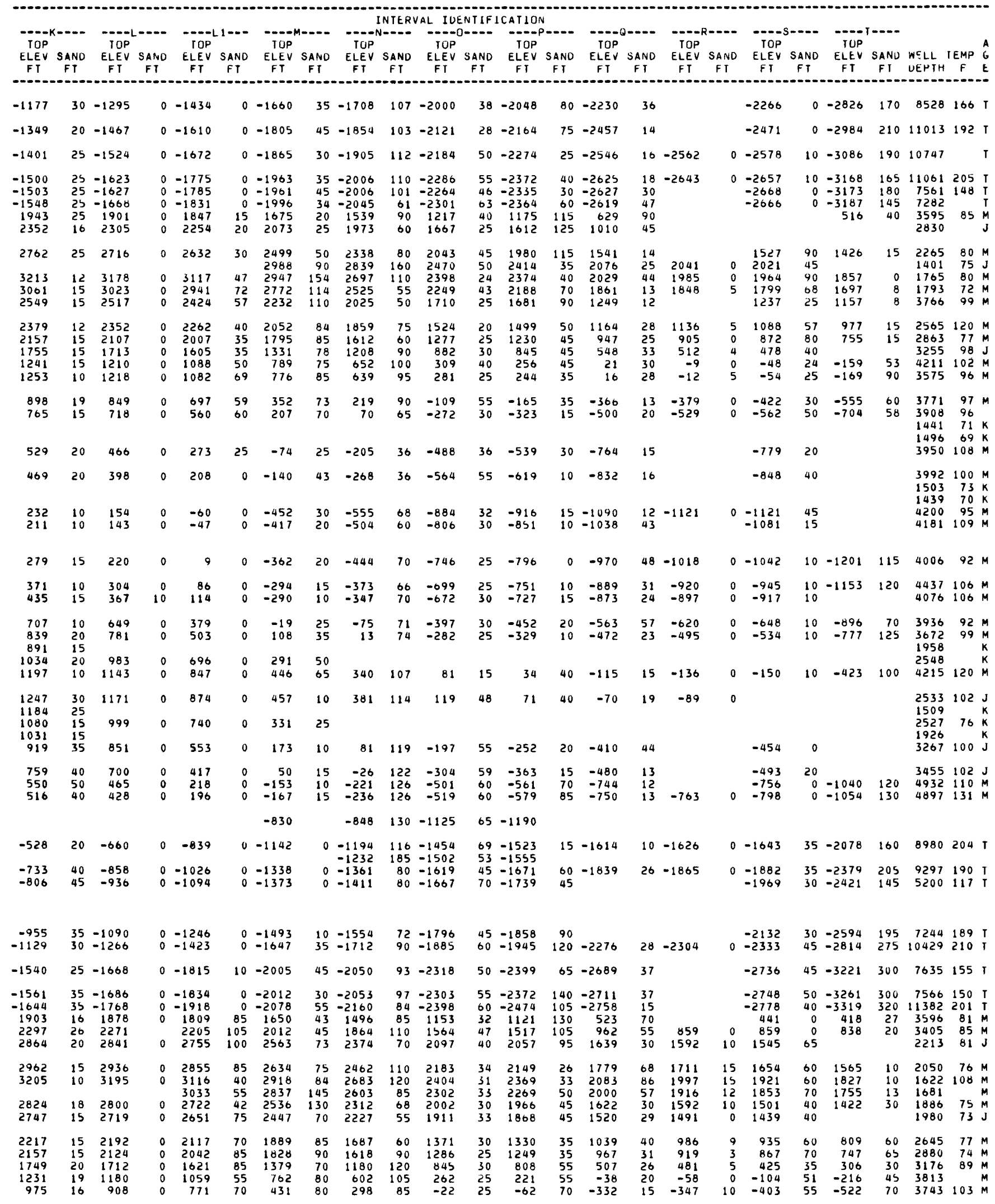


TABLE 2. - SELECIEU geolugic dATA -- CONTINUED

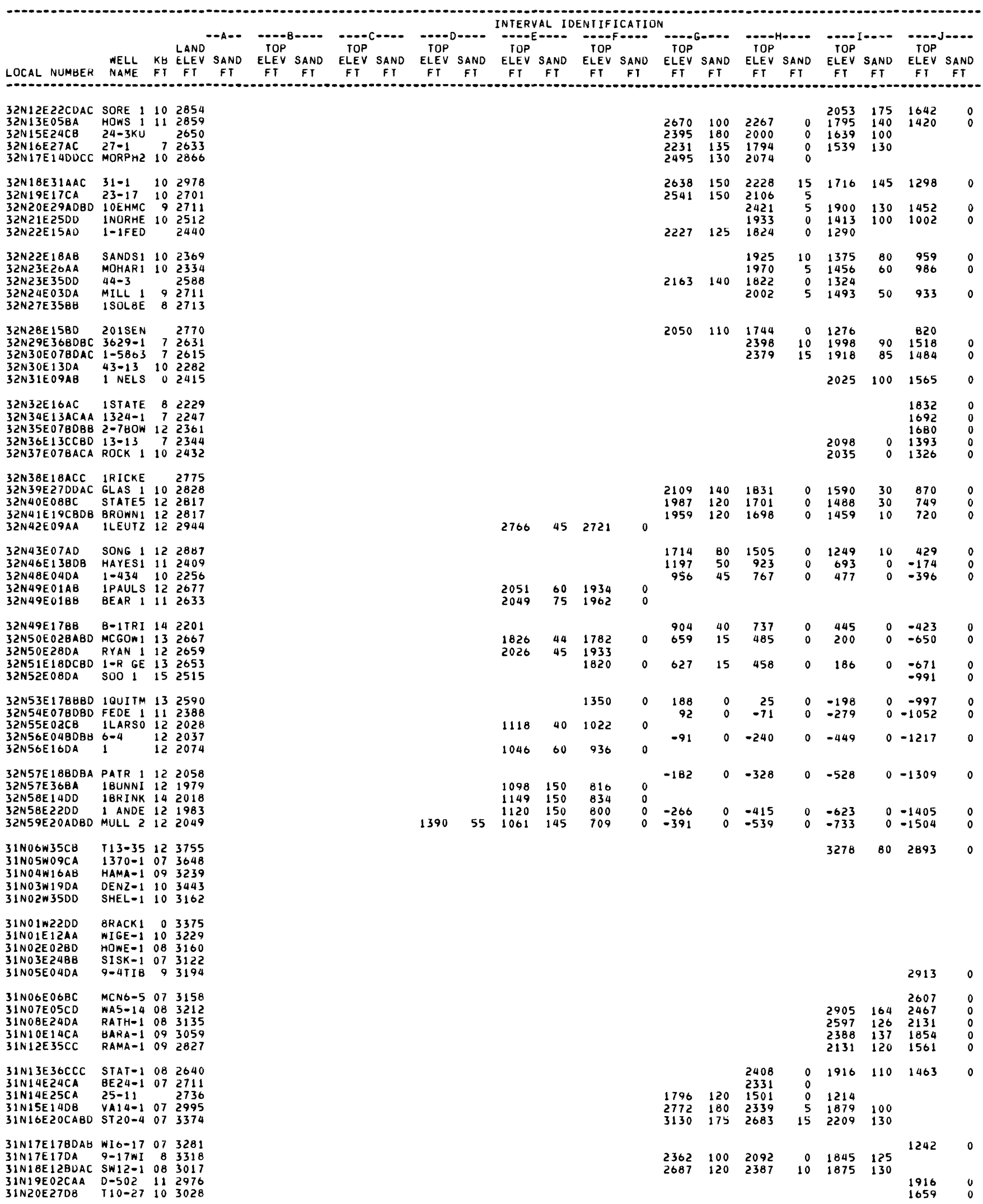




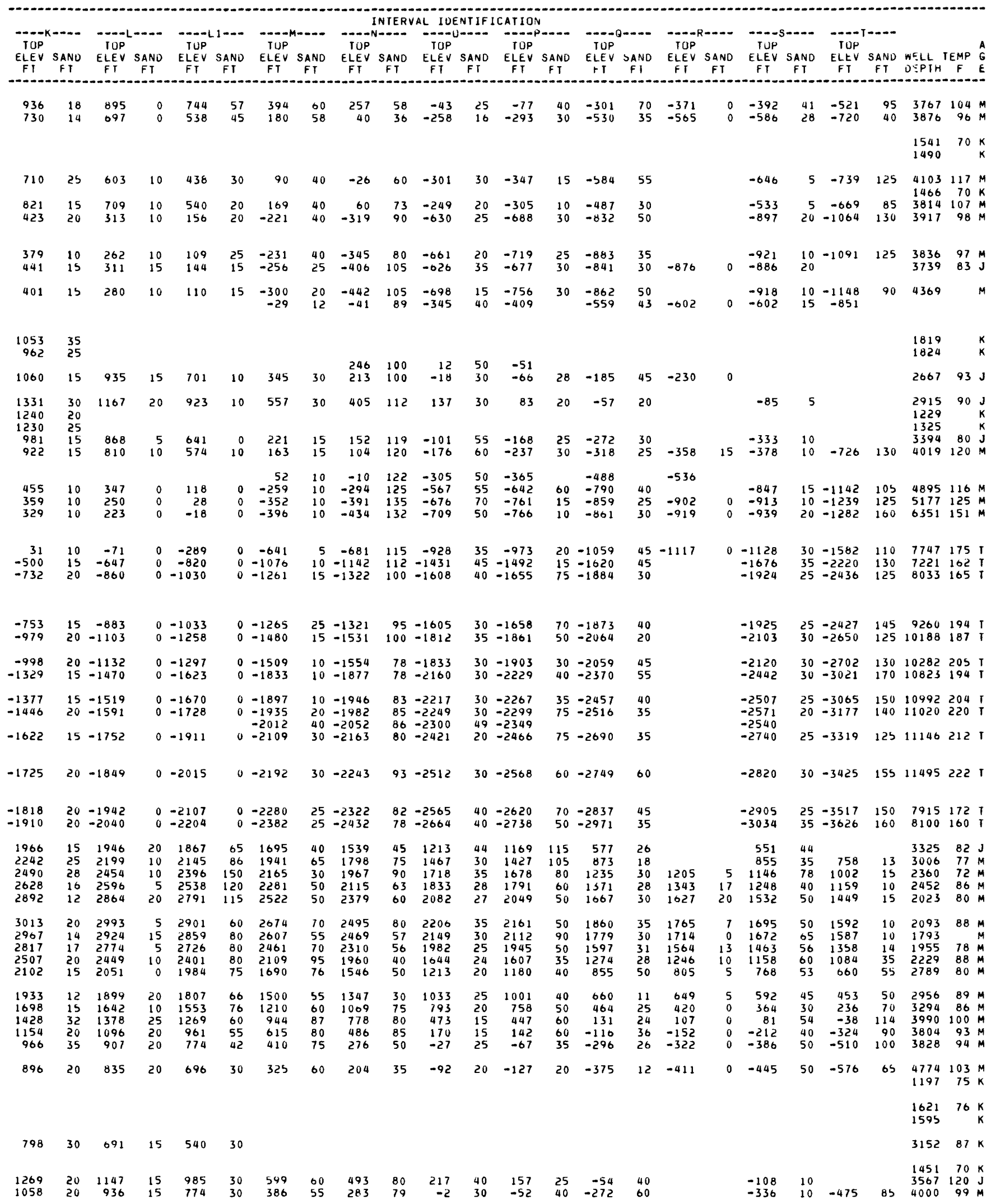


TABLE 2. -- SELECTED geOLOGIC dATA -- CUNTINUED

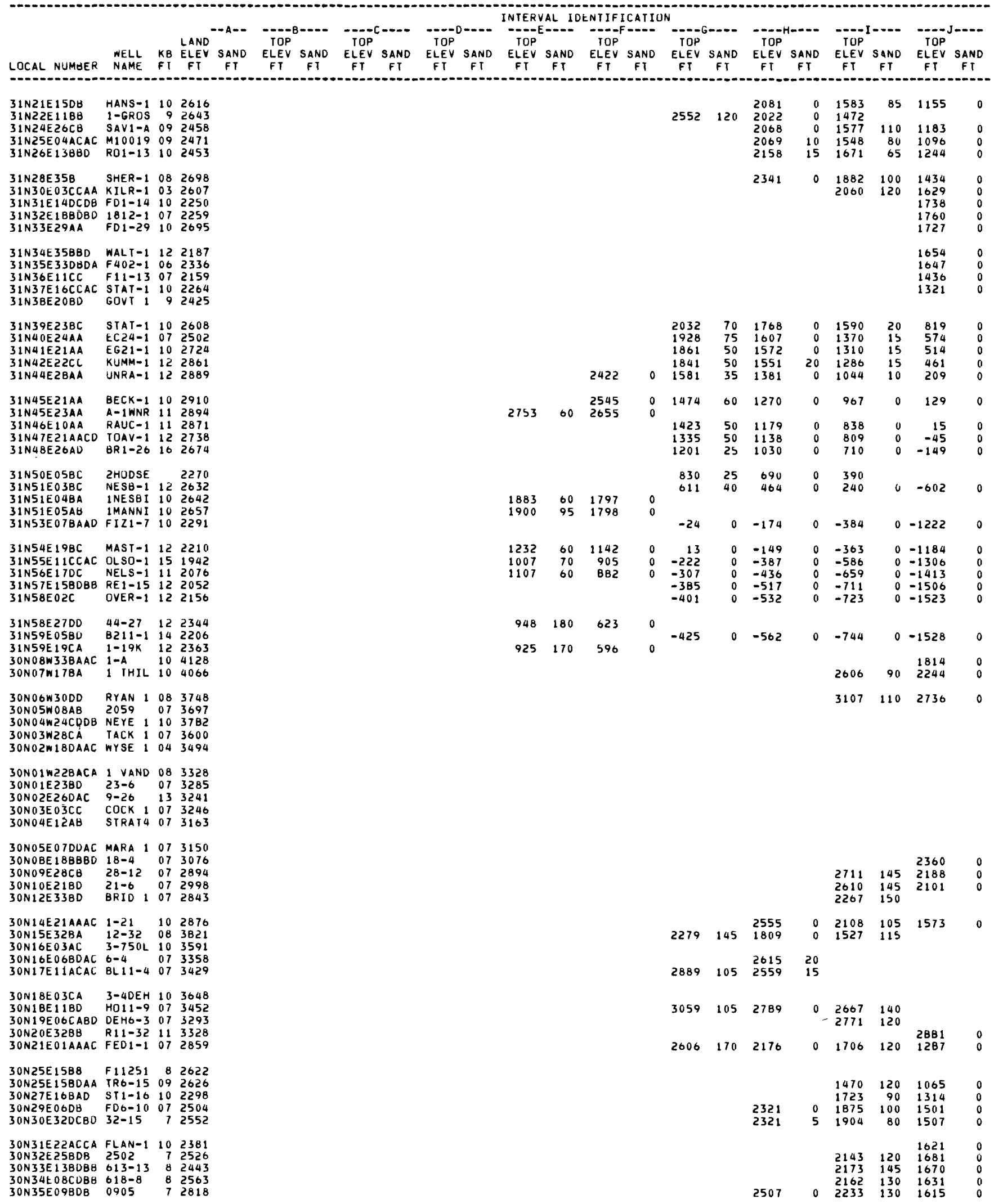




\begin{tabular}{|c|c|c|c|c|c|c|c|c|c|c|c|c|c|c|c|c|c|c|c|c|c|c|c|c|}
\hline $\begin{array}{l}\text { TOP } \\
\text { ELEV } \\
F T\end{array}$ & $\begin{array}{l}K=\ldots- \\
\text { SAND } \\
\text { FT }\end{array}$ & $\begin{array}{c}\text { IOP } \\
\text { ELEV } \\
F T\end{array}$ & $\begin{array}{c}\text { SAND } \\
\text { FT }\end{array}$ & $\begin{array}{c}\text { TUP } \\
\text { ELEV } \\
\text { FT }\end{array}$ & $\begin{array}{c}1=- \\
\text { SAND } \\
\text { FI }\end{array}$ & $\begin{array}{l}\text { IOP } \\
\text { ELEV } \\
\text { FI }\end{array}$ & $\begin{array}{c}\text { SAND } \\
\text { FT }\end{array}$ & $\begin{array}{l}\text { TOP } \\
\text { ELEV } \\
\text { FT }\end{array}$ & $\begin{array}{l}\text { SAND } \\
\text { FT }\end{array}$ & $\begin{array}{c}\text { IUP } \\
\text { ELEV } \\
\text { FT }\end{array}$ & $\begin{array}{l}\text { SAND } \\
\text { FI }\end{array}$ & $\begin{array}{l}\text { TOP } \\
\text { ELEV }\end{array}$ & $\begin{array}{c}\text { SAND } \\
\text { FT }\end{array}$ & $\begin{array}{c}\text { IOP } \\
\text { ELEV } \\
F T\end{array}$ & $\begin{array}{c}\text { SANO } \\
\text { FI }\end{array}$ & $\begin{array}{c}\text { IUP } \\
\text { ELEV } \\
F I\end{array}$ & $\begin{array}{l}\text { SAND } \\
\text { FT }\end{array}$ & $\begin{array}{c}\text { IUP } \\
\text { ELEV } \\
F I\end{array}$ & $\begin{array}{c}S=-. \\
\text { SAND } \\
\text { FT }\end{array}$ & $\begin{array}{c}\text { TUP } \\
\text { ELEV } \\
\text { F }\end{array}$ & $\begin{array}{l}\text { SANO } \\
\text { FT }\end{array}$ & $\begin{array}{l}\text { WELL } \\
\text { DEP TH }\end{array}$ & $\begin{array}{c}\text { TEMP } \\
\mathbf{F}\end{array}$ & $\begin{array}{l}A \\
6 \\
E\end{array}$ \\
\hline $\begin{array}{l}588 \\
603 \\
585 \\
725\end{array}$ & $\begin{array}{l}15 \\
20 \\
30 \\
35\end{array}$ & $\begin{array}{l}497 \\
506 \\
486 \\
604\end{array}$ & $\begin{array}{l}10 \\
10 \\
25 \\
25\end{array}$ & $\begin{array}{l}306 \\
293 \\
263 \\
375\end{array}$ & $\begin{array}{l}20 \\
35 \\
20 \\
25\end{array}$ & $\begin{array}{r}-74 \\
-117 \\
-150 \\
-38\end{array}$ & $\begin{array}{l}40 \\
35 \\
20 \\
20\end{array}$ & $\begin{array}{l}-173 \\
-285 \\
-254 \\
-286\end{array}$ & $\begin{array}{r}84 \\
93 \\
104 \\
81\end{array}$ & $\begin{array}{l}-490 \\
-611 \\
-514 \\
-519\end{array}$ & $\begin{array}{l}20 \\
30 \\
30 \\
15\end{array}$ & $\begin{array}{l}-547 \\
-601 \\
-546 \\
-577\end{array}$ & $\begin{array}{l}15 \\
25 \\
40\end{array}$ & $\begin{array}{l}-729 \\
-783 \\
-761 \\
-758\end{array}$ & $\begin{array}{l}70 \\
30 \\
35\end{array}$ & $\begin{array}{l}-816 \\
-795\end{array}$ & 0 & $\begin{array}{l}-804 \\
-813 \\
-801\end{array}$ & $\begin{array}{l}10 \\
30 \\
15\end{array}$ & $\begin{array}{r}-995 \\
-1015\end{array}$ & $\begin{array}{l}85 \\
85\end{array}$ & $\begin{array}{l}4890 \\
3894 \\
2043\end{array}$ & $\begin{array}{rr}4 & 105 \\
0 & 102 \\
4 & 97 \\
3 & 79\end{array}$ & $\begin{array}{l}M \\
M\end{array}$ \\
\hline $\begin{array}{r}927 \\
1099 \\
1202 \\
1227 \\
1206\end{array}$ & $\begin{array}{l}35 \\
50 \\
45 \\
30 \\
20\end{array}$ & $\begin{array}{r}789 \\
1053 \\
1090\end{array}$ & $\begin{array}{l}20 \\
20\end{array}$ & $\begin{array}{l}553 \\
811 \\
849\end{array}$ & $\begin{array}{l}10 \\
10\end{array}$ & $\begin{array}{l}138 \\
448 \\
495\end{array}$ & $\begin{array}{l}25 \\
35 \\
30\end{array}$ & $\begin{array}{l}284 \\
374\end{array}$ & 110 & $\begin{array}{l}44 \\
98\end{array}$ & 40 & & & & & & & & & & & $\begin{array}{l}2710 \\
1849 \\
2366 \\
1222 \\
2692\end{array}$ & $\begin{array}{l}75 \\
84 \\
80\end{array}$ & $\begin{array}{l}k \\
k \\
k\end{array}$ \\
\hline $\begin{array}{l}1194 \\
1173 \\
1006 \\
904\end{array}$ & $\begin{array}{l}15 \\
20 \\
15 \\
15\end{array}$ & $\begin{array}{l}901 \\
799\end{array}$ & $\begin{array}{l}15 \\
10\end{array}$ & $\begin{array}{l}661 \\
555\end{array}$ & $\begin{array}{l}10 \\
10\end{array}$ & $\begin{array}{l}241 \\
144 \\
-96\end{array}$ & $\begin{array}{l}15 \\
20 \\
10\end{array}$ & $\begin{array}{r}184 \\
95 \\
-106\end{array}$ & $\begin{array}{l}120 \\
120 \\
128\end{array}$ & $\begin{array}{r}-79 \\
-169 \\
-394\end{array}$ & $\begin{array}{l}65 \\
55 \\
57\end{array}$ & $\begin{array}{l}-145 \\
-229 \\
-451\end{array}$ & $\begin{array}{l}60 \\
60\end{array}$ & $\begin{array}{l}-258 \\
-401 \\
-580\end{array}$ & $\begin{array}{l}35 \\
35 \\
68\end{array}$ & $\begin{array}{l}-298 \\
-441 \\
-654\end{array}$ & $\begin{array}{l}0 \\
0\end{array}$ & $\begin{array}{l}-310 \\
-460\end{array}$ & $\begin{array}{l}30 \\
30\end{array}$ & -768 & 80 & $\begin{array}{l}1242 \\
1448 \\
3195 \\
4219\end{array}$ & $\begin{array}{rr}2 & \\
8 & 70 \\
5 & 115 \\
9 & 125\end{array}$ & $\begin{array}{l}K \\
K \\
J \\
M\end{array}$ \\
\hline $\begin{array}{l}455 \\
191 \\
134 \\
113 \\
133\end{array}$ & $\begin{array}{l}15 \\
15 \\
20 \\
15 \\
20\end{array}$ & $\begin{array}{r}341 \\
66 \\
13 \\
-9 \\
-283\end{array}$ & $\begin{array}{r}10 \\
5 \\
0 \\
0 \\
0\end{array}$ & $\begin{array}{r}85 \\
-189 \\
-250 \\
-204 \\
-514\end{array}$ & $\begin{array}{r}10 \\
0 \\
0 \\
0 \\
0\end{array}$ & $\begin{array}{l}-256 \\
-535 \\
-574 \\
-555 \\
-807\end{array}$ & $\begin{array}{l}15 \\
10 \\
10 \\
15 \\
15\end{array}$ & $\begin{array}{l}-312 \\
-591 \\
-627 \\
-633 \\
-880\end{array}$ & $\begin{array}{l}129 \\
135 \\
128 \\
134 \\
125\end{array}$ & $\begin{array}{r}-591 \\
-876 \\
-906 \\
-921 \\
-1143\end{array}$ & $\begin{array}{l}50 \\
30 \\
40 \\
05 \\
35\end{array}$ & $\begin{array}{r}-645 \\
-927 \\
-960 \\
-996 \\
-1199\end{array}$ & $\begin{array}{l}20 \\
15 \\
15 \\
25 \\
35\end{array}$ & $\begin{array}{r}-773 \\
-1053 \\
-1088 \\
-1105 \\
-1296\end{array}$ & $\begin{array}{l}35 \\
20 \\
25 \\
30 \\
40\end{array}$ & $\begin{array}{r}-815 \\
-1081\end{array}$ & $\begin{array}{l}0 \\
0\end{array}$ & $\begin{array}{r}-835 \\
-11102 \\
-11126 \\
-11137 \\
-1364\end{array}$ & $\begin{array}{l}40 \\
15 \\
25 \\
30 \\
25\end{array}$ & $\begin{array}{l}-1218 \\
-1491 \\
-1554 \\
-1568 \\
-1847\end{array}$ & $\begin{array}{l}90 \\
130 \\
115 \\
130 \\
130\end{array}$ & $\begin{array}{l}4499 \\
5003 \\
5316 \\
6776 \\
5580\end{array}$ & $\begin{array}{ll}9 & 121 \\
3 & 135 \\
b & 140 \\
b & 145 \\
0 & 140\end{array}$ & $\begin{array}{l}M \\
M \\
M \\
M \\
T\end{array}$ \\
\hline-198 & 30 & -344 & 0 & -544 & 0 & -835 & 15 & -900 & 110 & -1161 & 35 & -1207 & 90 & -1345 & 35 & & & -1400 & 35 & -1915 & 115 & 5684 & 133 & $\mathbf{T}$ \\
\hline $\begin{array}{l}-325 \\
-385 \\
-480\end{array}$ & $\begin{array}{l}20 \\
20 \\
25\end{array}$ & $\begin{array}{l}-476 \\
-534 \\
-619\end{array}$ & $\begin{array}{l}0 \\
0 \\
0\end{array}$ & $\begin{array}{l}-669 \\
-735 \\
-805\end{array}$ & $\begin{array}{l}0 \\
0 \\
0\end{array}$ & $\begin{array}{l}-989 \\
-1060 \\
-1090\end{array}$ & $\begin{array}{l}10 \\
10 \\
10\end{array}$ & 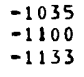 & $\begin{array}{r}115 \\
110 \\
85\end{array}$ & $\begin{array}{l}-1286 \\
-1349 \\
-1376\end{array}$ & $\begin{array}{l}30 \\
45 \\
35\end{array}$ & $\begin{array}{l}-1334 \\
-1406 \\
-1441\end{array}$ & $\begin{array}{l}50 \\
35 \\
50\end{array}$ & $\begin{array}{l}-1433 \\
-1513 \\
-1621\end{array}$ & $\begin{array}{l}30 \\
25 \\
35\end{array}$ & & & $\begin{array}{l}-1484 \\
-1568 \\
-1676\end{array}$ & $\begin{array}{l}50 \\
40 \\
35\end{array}$ & $\begin{array}{l}-2014 \\
-2074 \\
-2169\end{array}$ & $\begin{array}{l}130 \\
135 \\
130\end{array}$ & $\begin{array}{l}7611 \\
7720 \\
7600\end{array}$ & $\begin{array}{l}175 \\
0 \\
0 \\
0 \\
160 \\
164\end{array}$ & $\begin{array}{l}T \\
1 \\
1\end{array}$ \\
\hline-948 & 20 & -1100 & 0 & -1256 & 0 & -1460 & 10 & -1506 & 76 & -1771 & 25 & -1822 & 75 & -2074 & 30 & & & -2129 & 30 & -2018 & 140 & 9056 & 6204 & 1 \\
\hline-1573 & 20 & -1728 & 0 & -1889 & 0 & -2104 & 15 & -2153 & 110 & -2415 & 25 & -2464 & 60 & -2717 & 30 & & & -2765 & 20 & -3291 & 140 & 10812 & 2203 & $T$ \\
\hline $\begin{array}{l}-1564 \\
-1753 \\
-1834 \\
-1900 \\
-1913\end{array}$ & $\begin{array}{l}20 \\
20 \\
20 \\
30 \\
20\end{array}$ & $\begin{array}{l}-1704 \\
-1901 \\
-1969 \\
-2046 \\
-2047\end{array}$ & $\begin{array}{l}0 \\
0 \\
0 \\
0 \\
0\end{array}$ & $\begin{array}{l}-1871 \\
-2063 \\
-2160 \\
-2209 \\
-2205\end{array}$ & $\begin{array}{l}0 \\
0 \\
0 \\
0 \\
0\end{array}$ & $\begin{array}{l}-2080 \\
-2258 \\
-2307 \\
-2386 \\
-2384\end{array}$ & $\begin{array}{l}10 \\
20 \\
25 \\
40 \\
70\end{array}$ & $\begin{array}{l}-2126 \\
-2299 \\
-2368 \\
-2438 \\
-2408\end{array}$ & $\begin{array}{r}106 \\
102 \\
85 \\
80 \\
72\end{array}$ & $\begin{array}{l}-2385 \\
-2568 \\
-2678 \\
-2691 \\
-2703\end{array}$ & $\begin{array}{l}35 \\
35 \\
30 \\
40 \\
40\end{array}$ & $\begin{array}{l}-2446 \\
-2637 \\
-2733 \\
-2753 \\
-2763\end{array}$ & $\begin{array}{l}50 \\
65 \\
50 \\
45 \\
50\end{array}$ & $\begin{array}{l}-2727 \\
-2848 \\
-2941 \\
-3015 \\
-3014\end{array}$ & $\begin{array}{l}40 \\
35 \\
45 \\
30 \\
45\end{array}$ & & & $\begin{array}{l}-2777 \\
-2907 \\
-3010 \\
-3080 \\
-3081\end{array}$ & $\begin{array}{l}30 \\
30 \\
25 \\
30 \\
30\end{array}$ & $\begin{array}{l}-3288 \\
-3469 \\
-3553 \\
-3622 \\
-3637\end{array}$ & $\begin{array}{l}115 \\
135 \\
150 \\
130 \\
125\end{array}$ & $\begin{array}{r}10957 \\
11361 \\
8341 \\
8138 \\
12097\end{array}$ & $\begin{array}{l}218 \\
223 \\
168 \\
180 \\
235\end{array}$ & $\begin{array}{l}T \\
T \\
T \\
T \\
T\end{array}$ \\
\hline-1914 & 20 & -2054 & 0 & -2205 & 0 & -2404 & 40 & -2474 & 90 & -2724 & 25 & -2774 & 40 & -3025 & 35 & & & -3088 & .30 & -3651 & 115 & 12157 & 220 & $T$ \\
\hline $\begin{array}{r}792 \\
1248\end{array}$ & $\begin{array}{l}15 \\
14\end{array}$ & $\begin{array}{r}764 \\
1220\end{array}$ & $\begin{array}{l}10 \\
10\end{array}$ & $\begin{array}{r}705 \\
1169\end{array}$ & $\begin{array}{l}48 \\
35\end{array}$ & $\begin{array}{l}425 \\
911\end{array}$ & $\begin{array}{l}15 \\
16\end{array}$ & $\begin{array}{l}368 \\
854\end{array}$ & $\begin{array}{l}50 \\
33\end{array}$ & $\begin{array}{r}46 \\
524\end{array}$ & $\begin{array}{l}30 \\
30\end{array}$ & $\begin{array}{r}10 \\
464\end{array}$ & $\begin{array}{r}110 \\
55\end{array}$ & $\begin{array}{l}-702 \\
-213\end{array}$ & $\begin{array}{l}65 \\
80\end{array}$ & & & $\begin{array}{l}-794 \\
-293\end{array}$ & $\begin{array}{l}0 \\
0\end{array}$ & $\begin{array}{l}-849 \\
-352\end{array}$ & $\begin{array}{l}50 \\
23\end{array}$ & $\begin{array}{l}5221 \\
4665\end{array}$ & $\begin{array}{r}104 \\
58\end{array}$ & $M$ \\
\hline $\begin{array}{l}1786 \\
2190 \\
2673 \\
2745 \\
2824\end{array}$ & $\begin{array}{l}18 \\
13 \\
12 \\
14 \\
15\end{array}$ & $\begin{array}{l}1748 \\
2166 \\
2650 \\
2722 \\
2802\end{array}$ & $\begin{array}{r}10 \\
10 \\
0 \\
5 \\
0\end{array}$ & $\begin{array}{l}1699 \\
2087 \\
2574 \\
2651 \\
2735\end{array}$ & $\begin{array}{l}67 \\
60 \\
58 \\
47 \\
65\end{array}$ & $\begin{array}{l}1450 \\
1816 \\
2269 \\
2320 \\
2361\end{array}$ & $\begin{array}{l}34 \\
34 \\
30 \\
31 \\
36\end{array}$ & $\begin{array}{l}1362 \\
1748 \\
2194 \\
2240 \\
2278\end{array}$ & $\begin{array}{l}70 \\
48 \\
35 \\
30 \\
28\end{array}$ & $\begin{array}{l}1024 \\
1427 \\
1875 \\
1959 \\
2009\end{array}$ & $\begin{array}{l}45 \\
35 \\
30 \\
30 \\
45\end{array}$ & $\begin{array}{l}962 \\
1369 \\
1834 \\
1909 \\
1945\end{array}$ & $\begin{array}{l}50 \\
41 \\
60 \\
50 \\
48\end{array}$ & $\begin{array}{r}356 \\
887 \\
1402 \\
1483 \\
1565\end{array}$ & $\begin{array}{l}80 \\
20 \\
12 \\
10 \\
19\end{array}$ & $\begin{array}{l}866 \\
1390 \\
1473 \\
1546\end{array}$ & $\begin{array}{l}0 \\
0\end{array}$ & $\begin{array}{r}274 \\
810 \\
1302 \\
1398 \\
1470\end{array}$ & $\begin{array}{l}0 \\
76 \\
50 \\
40 \\
45\end{array}$ & $\begin{array}{r}216 \\
664 \\
1195 \\
1285 \\
1361\end{array}$ & $\begin{array}{r}13 \\
10 \\
8 \\
6 \\
8\end{array}$ & $\begin{array}{l}3762 \\
3296 \\
2670 \\
2401 \\
2230\end{array}$ & $\begin{array}{l}75 \\
b \\
69 \\
79 \\
67 \\
62\end{array}$ & $\begin{array}{l}M \\
M \\
M \\
M \\
M\end{array}$ \\
\hline $\begin{array}{l}2945 \\
2867 \\
2683 \\
2527 \\
2198\end{array}$ & $\begin{array}{l}15 \\
16 \\
15 \\
18 \\
25\end{array}$ & $\begin{array}{l}2914 \\
2829 \\
2651 \\
2481 \\
2142\end{array}$ & $\begin{array}{l}5 \\
0 \\
0 \\
5 \\
5\end{array}$ & $\begin{array}{l}2831 \\
2789 \\
2605 \\
2417 \\
2007\end{array}$ & $\begin{array}{l}80 \\
74 \\
69 \\
55 \\
46\end{array}$ & $\begin{array}{l}2484 \\
2420 \\
2322 \\
2145 \\
1830\end{array}$ & $\begin{array}{r}37 \\
40 \\
65 \\
65 \\
108\end{array}$ & $\begin{array}{l}2387 \\
2326 \\
2142 \\
1993 \\
1612\end{array}$ & $\begin{array}{l}27 \\
80 \\
60 \\
45 \\
47\end{array}$ & $\begin{array}{l}2111 \\
2034 \\
1842 \\
1689 \\
1305\end{array}$ & $\begin{array}{l}30 \\
23 \\
36 \\
25 \\
20\end{array}$ & $\begin{array}{l}2062 \\
1988 \\
1802 \\
1648 \\
1273\end{array}$ & $\begin{array}{l}40 \\
55 \\
38 \\
60 \\
60\end{array}$ & $\begin{array}{r}1696 \\
1625 \\
1422 \\
1271 \\
920\end{array}$ & $\begin{array}{l}15 \\
17 \\
18 \\
28 \\
37\end{array}$ & $\begin{array}{r}1062 \\
1597 \\
1404 \\
1243 \\
883\end{array}$ & $\begin{array}{l}0 \\
0 \\
0 \\
5 \\
0\end{array}$ & $\begin{array}{r}1003 \\
1532 \\
1352 \\
1205 \\
842\end{array}$ & $\begin{array}{l}45 \\
68 \\
80 \\
80 \\
67\end{array}$ & $\begin{array}{r}1480 \\
1437 \\
1248 \\
1103 \\
730\end{array}$ & $\begin{array}{l}10 \\
20 \\
20 \\
20 \\
15\end{array}$ & $\begin{array}{l}1990 \\
2019 \\
2208 \\
2359 \\
2752\end{array}$ & $\begin{array}{ll}0 & 87 \\
9 & 75 \\
3 & 77 \\
7 & 79 \\
? & 78\end{array}$ & $\begin{array}{l}M \\
M \\
M \\
M \\
M\end{array}$ \\
\hline $\begin{array}{l}2151 \\
1626 \\
1502 \\
1420\end{array}$ & $\begin{array}{l}25 \\
20 \\
19 \\
30\end{array}$ & $\begin{array}{l}2095 \\
1576 \\
1453 \\
1369\end{array}$ & $\begin{array}{r}5 \\
5 \\
10 \\
10\end{array}$ & $\begin{array}{l}2022 \\
1473 \\
1341 \\
1254\end{array}$ & $\begin{array}{l}50 \\
62 \\
65 \\
69\end{array}$ & $\begin{array}{r}1781 \\
1190 \\
1059 \\
959\end{array}$ & $\begin{array}{r}95 \\
90 \\
70 \\
110\end{array}$ & $\begin{array}{r}1560 \\
980 \\
841 \\
741\end{array}$ & $\begin{array}{l}35 \\
45 \\
50 \\
50\end{array}$ & $\begin{array}{r}1260 \\
675 \\
551 \\
455\end{array}$ & $\begin{array}{l}23 \\
20 \\
21 \\
20\end{array}$ & $\begin{array}{r}1225 \\
600 \\
511 \\
422\end{array}$ & $\begin{array}{l}35 \\
20 \\
40 \\
10\end{array}$ & $\begin{array}{l}889 \\
401 \\
268 \\
158\end{array}$ & $\begin{array}{l}60 \\
26 \\
30 \\
16\end{array}$ & $\begin{array}{l}829 \\
375 \\
221 \\
142\end{array}$ & $\begin{array}{l}0 \\
0 \\
0 \\
0\end{array}$ & $\begin{array}{r}777 \\
336 \\
141 \\
49\end{array}$ & $\begin{array}{l}71 \\
30 \\
30 \\
27\end{array}$ & $\begin{array}{r}679 \\
239 \\
41 \\
-65\end{array}$ & $\begin{array}{l}20 \\
25 \\
30 \\
45\end{array}$ & $\begin{array}{r}2751 \\
4939 \\
3195 \\
3340 \\
995\end{array}$ & $\begin{array}{r}83 \\
98 \\
117 \\
118\end{array}$ & $\begin{array}{l}M \\
M \\
M \\
M\end{array}$ \\
\hline \multirow[t]{3}{*}{1035} & 25 & 983 & 20 & 846 & 63 & 475 & 43 & 353 & 40 & 58 & 50 & 3 & 30 & -207 & 26 & -233 & 0 & -279 & 40 & -413 & 55 & $\begin{array}{l}3657 \\
2705\end{array}$ & $\begin{array}{r}120 \\
76\end{array}$ & $\begin{array}{l}M \\
x\end{array}$ \\
\hline & & & & & & & & 351 & 33 & 68 & 30 & 13 & & -191 & 42 & -233 & & -267 & 70 & -411 & & $\begin{array}{l}1495 \\
1620\end{array}$ & 75 & $K$ \\
\hline & & & & & & & & 708 & 60 & 430 & 40 & 369 & & 158 & 20 & 130 & & 109 & 65 & -45 & & $\begin{array}{l}1193 \\
1144\end{array}$ & $\begin{array}{l}62 \\
67\end{array}$ & $\begin{array}{l}k \\
k\end{array}$ \\
\hline $\begin{array}{r}2324 \\
698\end{array}$ & $\begin{array}{l}40 \\
20\end{array}$ & $\begin{array}{r}2207 \\
616\end{array}$ & $\begin{array}{r}5 \\
10\end{array}$ & $\begin{array}{r}2006 \\
394\end{array}$ & $\begin{array}{l}45 \\
25\end{array}$ & $\begin{array}{r}1589 \\
11\end{array}$ & $\begin{array}{l}45 \\
40\end{array}$ & $\begin{array}{r}1486 \\
-91\end{array}$ & $\begin{array}{l}77 \\
59\end{array}$ & $\begin{array}{l}1208 \\
-393\end{array}$ & $\begin{array}{l}20 \\
40\end{array}$ & $\begin{array}{l}1157 \\
-464\end{array}$ & $\begin{array}{l}30 \\
35\end{array}$ & $\begin{array}{r}897 \\
-684\end{array}$ & $\begin{array}{l}30 \\
40\end{array}$ & & & $\begin{array}{r}859 \\
-724\end{array}$ & $\begin{array}{l}10 \\
35\end{array}$ & -889 & 90 & & $\begin{array}{l}80 \\
97\end{array}$ & $\begin{array}{l}\mathrm{J} \\
\mathrm{M}\end{array}$ \\
\hline $\begin{array}{l}517 \\
774 \\
912 \\
929\end{array}$ & $\begin{array}{l}35 \\
25 \\
40 \\
40\end{array}$ & $\begin{array}{l}777 \\
799\end{array}$ & $\begin{array}{l}20 \\
10\end{array}$ & $\begin{array}{l}547 \\
562\end{array}$ & $\begin{array}{l}15 \\
15\end{array}$ & $\begin{array}{l}201 \\
209\end{array}$ & $\begin{array}{l}25 \\
30\end{array}$ & $\begin{array}{l}32 \\
51\end{array}$ & $\begin{array}{l}94 \\
96\end{array}$ & $\begin{array}{l}-211 \\
-191\end{array}$ & $\begin{array}{l}35 \\
40\end{array}$ & -570 & 5 & -682 & 133 & -473 & 0 & -815 & 15 & -1000 & & $\begin{array}{l}2350 \\
1860 \\
2894 \\
3426\end{array}$ & 114 & K \\
\hline $\begin{array}{l}1066 \\
1124 \\
1140 \\
1103 \\
1126\end{array}$ & $\begin{array}{l}40 \\
55 \\
25 \\
25 \\
20\end{array}$ & 933 & 10 & 684 & 15 & 326 & 45 & 191 & 100 & -56 & 40 & -116 & 20 & -342 & 35 & -379 & 0 & -389 & 10 & -629 & 140 & $\begin{array}{l}3396 \\
1577 \\
1682 \\
1730 \\
1866\end{array}$ & 94 & $\begin{array}{l}M \\
k \\
k \\
k \\
K\end{array}$ \\
\hline
\end{tabular}




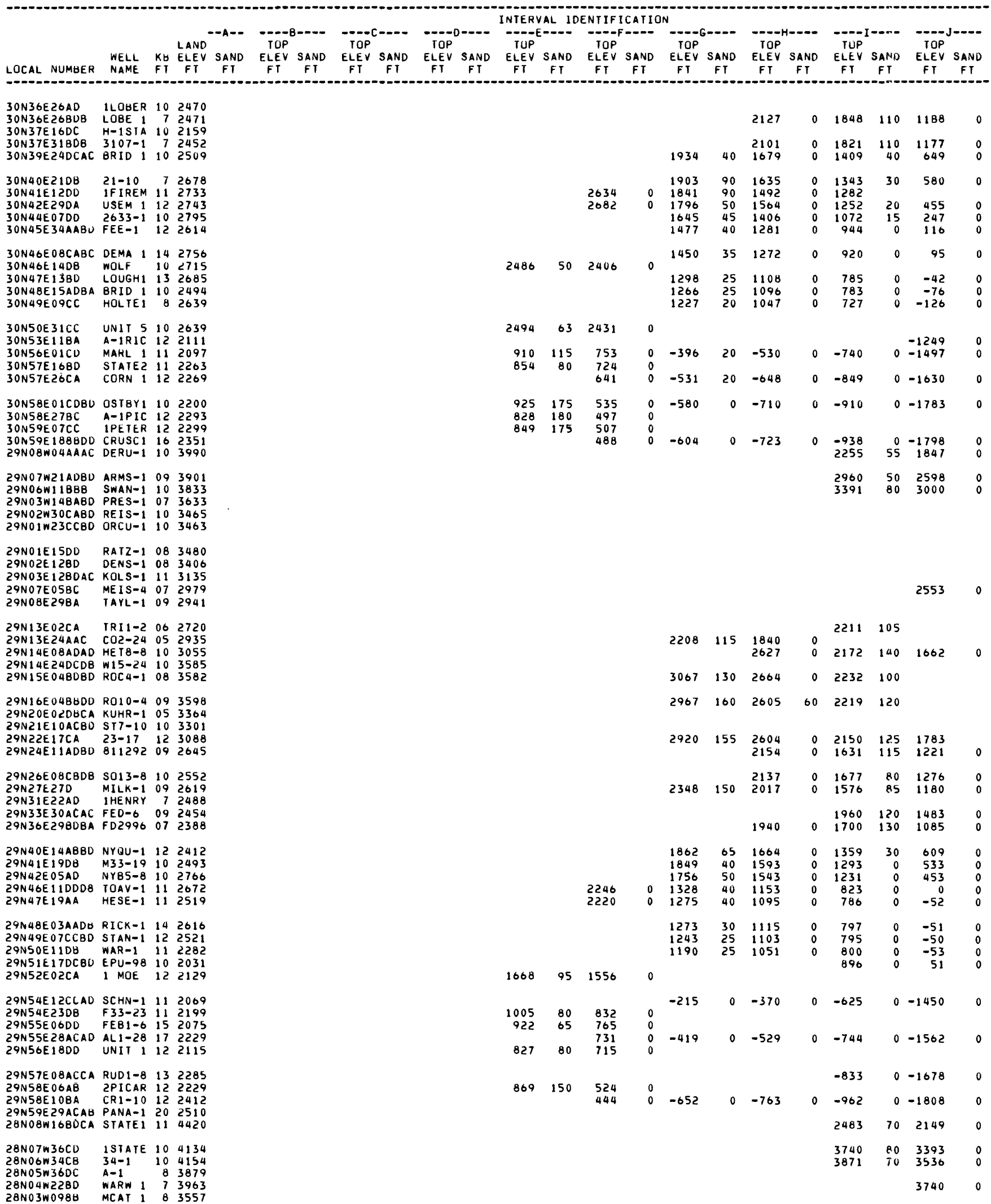




\begin{tabular}{|c|c|c|c|c|c|c|c|c|c|c|c|c|c|c|c|c|c|c|c|c|c|c|c|c|}
\hline 718 & 15 & & & & & & 22 & -41 & 111 & -292 & & -364 & & -485 & & -560 & 0 & & 0 & & & 1896 & & $k$ \\
\hline & & & & & & -31 & 30 & -61 & 153 & -311 & 40 & -371 & 45 & -561 & 38 & -599 & & -614 & & & & 1867 & & $k$ \\
\hline 249 & 15 & 109 & 0 & -121 & 0 & -390 & 51 & -441 & 130 & -691 & 35 & -751 & 15 & -853 & 25 & -903 & 0 & -918 & 20 & -1341 & 110 & 5245 & 137 & $\hat{M}$ \\
\hline 179 & 15 & 45 & 0 & -200 & 0 & -547 & 15 & -615 & 130 & -870 & 55 & -935 & 25 & -1027 & 30 & & & -1087 & 15 & -1525 & 135 & 4992 & 129 & M \\
\hline $\begin{array}{r}57 \\
-155 \\
-276\end{array}$ & $\begin{array}{l}20 \\
20 \\
35\end{array}$ & $\begin{array}{l}-60 \\
-265 \\
-410\end{array}$ & $\begin{array}{l}0 \\
0 \\
0\end{array}$ & $\begin{array}{l}-297 \\
-523 \\
-654\end{array}$ & $\begin{array}{l}0 \\
0 \\
0\end{array}$ & $\begin{array}{l}-645 \\
-847 \\
-951\end{array}$ & $\begin{array}{l}20 \\
15 \\
10\end{array}$ & $\begin{array}{l}-717 \\
-895 \\
-996\end{array}$ & $\begin{array}{l}120 \\
138 \\
110\end{array}$ & $\begin{array}{r}-976 \\
-1185 \\
-1264\end{array}$ & $\begin{array}{l}60 \\
35 \\
35\end{array}$ & $\begin{array}{l}-1040 \\
-1238 \\
-1314\end{array}$ & $\begin{array}{l}25 \\
20 \\
35\end{array}$ & $\begin{array}{l}-1142 \\
-1364 \\
-1519\end{array}$ & $\begin{array}{l}35 \\
45 \\
35\end{array}$ & & & $\begin{array}{l}-1194 \\
-1425 \\
-1594\end{array}$ & $\begin{array}{l}15 \\
30 \\
35\end{array}$ & $\begin{array}{l}-1645 \\
-1871 \\
-2054\end{array}$ & $\begin{array}{l}110 \\
135 \\
115\end{array}$ & $\begin{array}{l}8103 \\
8571 \\
7368\end{array}$ & $\begin{array}{l}174 \\
182 \\
100\end{array}$ & ' \\
\hline-260 & 35 & -398 & 0 & -608 & 0 & -920 & 10 & -970 & 95 & -1185 & 60 & -1249 & 100 & -1483 & 40 & & & -1537 & 40 & & & 5115 & 130 & $\mathbf{J}$ \\
\hline $\begin{array}{l}-375 \\
-415 \\
-479\end{array}$ & $\begin{array}{l}30 \\
25 \\
15\end{array}$ & $\begin{array}{l}-529 \\
-532 \\
-593\end{array}$ & $\begin{array}{l}0 \\
0 \\
0\end{array}$ & $\begin{array}{l}-722 \\
-728 \\
-784\end{array}$ & $\begin{array}{l}0 \\
0 \\
0\end{array}$ & $\begin{array}{r}-992 \\
-982 \\
-1003\end{array}$ & $\begin{array}{l}15 \\
15 \\
25\end{array}$ & $\begin{array}{l}-1062 \\
-1040 \\
-1069\end{array}$ & $\begin{array}{l}89 \\
76 \\
75\end{array}$ & $\begin{array}{l}-1281 \\
-1258 \\
-1317\end{array}$ & $\begin{array}{l}45 \\
30 \\
30\end{array}$ & $\begin{array}{l}-1354 \\
-1323 \\
-1364\end{array}$ & $\begin{array}{l}60 \\
70 \\
70\end{array}$ & $\begin{array}{l}-1578 \\
-1566 \\
-1590\end{array}$ & $\begin{array}{l}35 \\
35 \\
50\end{array}$ & & & $\begin{array}{l}-1641 \\
-1626 \\
-1673\end{array}$ & $\begin{array}{l}30 \\
25 \\
40\end{array}$ & $\begin{array}{l}-2132 \\
-2126 \\
-2214\end{array}$ & $\begin{array}{l}115 \\
120 \\
130\end{array}$ & $\begin{array}{l}7714 \\
7718 \\
7971\end{array}$ & $\begin{array}{l}158 \\
170 \\
160\end{array}$ & $\begin{array}{l}T \\
\gamma \\
T\end{array}$ \\
\hline $\begin{array}{l}-1627 \\
-1932\end{array}$ & $\begin{array}{l}45 \\
30\end{array}$ & $\begin{array}{l}-1772 \\
-2061\end{array}$ & $\begin{array}{l}0 \\
0\end{array}$ & $\begin{array}{l}-1950 \\
-2262\end{array}$ & $\begin{array}{l}0 \\
0\end{array}$ & $\begin{array}{r}-943 \\
-2146 \\
-2442\end{array}$ & $\begin{array}{l}20 \\
15 \\
20\end{array}$ & $\begin{array}{r}-973 \\
-2193 \\
-2479\end{array}$ & $\begin{array}{l}70 \\
70 \\
80\end{array}$ & $\begin{array}{l}-1223 \\
-2445 \\
-2751\end{array}$ & $\begin{array}{l}30 \\
35\end{array}$ & $\begin{array}{l}-1304 \\
-2496 \\
-2813\end{array}$ & $\begin{array}{l}60 \\
35\end{array}$ & $\begin{array}{l}-2726 \\
-3051\end{array}$ & $\begin{array}{l}80 \\
55\end{array}$ & & & $\begin{array}{l}-2836 \\
-3137\end{array}$ & $\begin{array}{l}40 \\
35\end{array}$ & $\begin{array}{l}-3367 \\
-3671\end{array}$ & $\begin{array}{l}110 \\
150\end{array}$ & $\begin{array}{l}6828 \\
8240\end{array}$ & $\begin{array}{l}140 \\
174\end{array}$ & $T$ \\
\hline-2069 & 35 & -2212 & 0 & -2401 & 0 & -2579 & 25 & -2619 & 80 & -2881 & 45 & -2952 & 40 & -3188 & 65 & & & -3270 & 40 & -3840 & 145 & 8724 & 180 & 1 \\
\hline-2171 & 45 & -2333 & 0 & -2492 & 0 & -2672 & 20 & -2713 & 80 & -2990 & 40 & $-30 \leq 0$ & 55 & -3298 & 60 & & & -3380 & 50 & -3940 & 145 & 7604 & 182 & $T$ \\
\hline $\begin{array}{r}-2163 \\
887\end{array}$ & $\begin{array}{l}25 \\
23\end{array}$ & $\begin{array}{r}-2308 \\
864\end{array}$ & $\begin{array}{r}0 \\
35\end{array}$ & $\begin{array}{r}-2487 \\
806\end{array}$ & $\begin{array}{l}0 \\
15\end{array}$ & $\begin{array}{r}-2670 \\
553\end{array}$ & $\begin{array}{r}30 \\
7\end{array}$ & $\begin{array}{r}-2711 \\
526\end{array}$ & $\begin{array}{l}70 \\
85\end{array}$ & $\begin{array}{r}-2958 \\
124\end{array}$ & $\begin{array}{l}40 \\
34\end{array}$ & $\begin{array}{r}-3022 \\
90\end{array}$ & $\begin{array}{l}60 \\
30\end{array}$ & $\begin{array}{r}-3228 \\
-605\end{array}$ & $\begin{array}{l}75 \\
42\end{array}$ & & & $\begin{array}{r}-3350 \\
-689\end{array}$ & $\begin{array}{r}40 \\
0\end{array}$ & $\begin{array}{r}-3902 \\
-754\end{array}$ & $\begin{array}{r}130 \\
20\end{array}$ & $\begin{array}{r}12645 \\
5027\end{array}$ & $\begin{array}{r}210 \\
84\end{array}$ & M \\
\hline $\begin{array}{l}1620 \\
2062 \\
2824 \\
2883 \\
2938\end{array}$ & $\begin{array}{l}15 \\
19 \\
16 \\
14 \\
14\end{array}$ & $\begin{array}{l}1605 \\
2043 \\
2800 \\
2857 \\
2913\end{array}$ & $\begin{array}{l}45 \\
20 \\
10 \\
10 \\
15\end{array}$ & $\begin{array}{l}1545 \\
1984 \\
2735 \\
2791 \\
2838\end{array}$ & $\begin{array}{l}12 \\
40 \\
33 \\
25 \\
75\end{array}$ & $\begin{array}{l}1273 \\
1699 \\
2410 \\
2452 \\
2463\end{array}$ & $\begin{array}{l}11 \\
15 \\
35 \\
33 \\
22\end{array}$ & $\begin{array}{l}1237 \\
1658 \\
2337 \\
2388 \\
2354\end{array}$ & $\begin{array}{l}32 \\
37 \\
30 \\
32 \\
70\end{array}$ & $\begin{array}{r}892 \\
1325 \\
2053 \\
2105 \\
2073\end{array}$ & $\begin{array}{l}43 \\
30 \\
28 \\
27 \\
30\end{array}$ & $\begin{array}{r}827 \\
1263 \\
2010 \\
2065 \\
2040\end{array}$ & $\begin{array}{l}40 \\
35 \\
80 \\
32 \\
65\end{array}$ & $\begin{array}{l}162 \\
668 \\
1592 \\
1645 \\
1653\end{array}$ & $\begin{array}{l}60 \\
50 \\
14 \\
40 \\
20\end{array}$ & $\begin{array}{l}1549 \\
1581 \\
1633\end{array}$ & $\begin{array}{l}0 \\
0 \\
0\end{array}$ & $\begin{array}{r}80 \\
586 \\
1485 \\
1545 \\
1548\end{array}$ & $\begin{array}{r}0 \\
0 \\
45 \\
50 \\
45\end{array}$ & $\begin{array}{r}24 \\
530 \\
1364 \\
1455 \\
1463\end{array}$ & $\begin{array}{r}15 \\
12 \\
0 \\
0 \\
15\end{array}$ & $\begin{array}{l}4079 \\
3792 \\
2419 \\
2154 \\
2181\end{array}$ & $\begin{array}{r}108 \\
70 \\
85 \\
77 \\
75\end{array}$ & $\begin{array}{l}M \\
M \\
M \\
M\end{array}$ \\
\hline $\begin{array}{l}2871 \\
2656 \\
2377 \\
1798 \\
1721\end{array}$ & $\begin{array}{r}9 \\
19 \\
16 \\
35 \\
16\end{array}$ & $\begin{array}{l}2838 \\
2619 \\
2336 \\
1749 \\
1686\end{array}$ & $\begin{array}{r}5 \\
0 \\
10 \\
30 \\
10\end{array}$ & $\begin{array}{l}2789 \\
2570 \\
2274 \\
1637 \\
1580\end{array}$ & $\begin{array}{l}76 \\
58 \\
59 \\
59 \\
54\end{array}$ & $\begin{array}{l}2391 \\
2279 \\
2002 \\
1358 \\
1300\end{array}$ & $\begin{array}{l}18 \\
52 \\
75 \\
88 \\
40\end{array}$ & $\begin{array}{l}2282 \\
2095 \\
1833 \\
1140 \\
1090\end{array}$ & $\begin{array}{l}47 \\
60 \\
64 \\
55 \\
60\end{array}$ & $\begin{array}{l}2022 \\
1814 \\
1546 \\
836 \\
780\end{array}$ & $\begin{array}{l}16 \\
26 \\
24 \\
20 \\
15\end{array}$ & $\begin{array}{l}1976 \\
1769 \\
1496 \\
795 \\
750\end{array}$ & $\begin{array}{l}50 \\
50 \\
50 \\
30 \\
30\end{array}$ & $\begin{array}{l}1619 \\
1393 \\
1143 \\
546 \\
474\end{array}$ & $\begin{array}{l}15 \\
29 \\
29 \\
39 \\
34\end{array}$ & $\begin{array}{r}1596 \\
1364 \\
1114 \\
507 \\
440\end{array}$ & $\begin{array}{r}0 \\
0 \\
0 \\
30 \\
0\end{array}$ & $\begin{array}{l}1518 \\
1314 \\
1063 \\
403 \\
360\end{array}$ & $\begin{array}{l}50 \\
40 \\
45 \\
60 \\
40\end{array}$ & $\begin{array}{r}1436 \\
978 \\
285\end{array}$ & $\begin{array}{l}30 \\
25 \\
22\end{array}$ & $\begin{array}{l}2209 \\
2220 \\
2360 \\
2825 \\
2910\end{array}$ & $\begin{array}{l}78 \\
80 \\
77 \\
77 \\
83\end{array}$ & $\begin{array}{l}M \\
J \\
M \\
J \\
M\end{array}$ \\
\hline $\begin{array}{l}1092 \\
1297\end{array}$ & $\begin{array}{l}13 \\
24\end{array}$ & $\begin{array}{l}1053 \\
1268\end{array}$ & $\begin{array}{l}20 \\
15\end{array}$ & $\begin{array}{r}906 \\
1084\end{array}$ & $\begin{array}{l}44 \\
37\end{array}$ & $\begin{array}{l}533 \\
103\end{array}$ & $\begin{array}{l}57 \\
50\end{array}$ & $\begin{array}{l}433 \\
588\end{array}$ & $\begin{array}{l}45 \\
25\end{array}$ & $\begin{array}{l}113 \\
322\end{array}$ & $\begin{array}{l}18 \\
25\end{array}$ & $\begin{array}{r}65 \\
245\end{array}$ & $\begin{array}{l}15 \\
15\end{array}$ & $\begin{array}{r}-133 \\
60\end{array}$ & $\begin{array}{l}30 \\
33\end{array}$ & $\begin{array}{r}-171 \\
27\end{array}$ & $\begin{array}{l}0 \\
0\end{array}$ & -55 & 40 & -181 & 40 & $\begin{array}{l}1481 \\
1812 \\
3339 \\
4106 \\
1989\end{array}$ & $\begin{array}{l}73 \\
62 \\
86 \\
93\end{array}$ & $\begin{array}{l}k \\
k \\
J \\
M \\
k\end{array}$ \\
\hline $\begin{array}{l}2825 \\
2751\end{array}$ & $\begin{array}{l}25 \\
15\end{array}$ & $\begin{array}{l}2734 \\
2651\end{array}$ & $\begin{array}{r}10 \\
0\end{array}$ & $\begin{array}{l}2539 \\
2465\end{array}$ & $\begin{array}{l}35 \\
15\end{array}$ & $\begin{array}{l}2170 \\
2091\end{array}$ & $\begin{array}{l}85 \\
30\end{array}$ & $\begin{array}{l}2030 \\
1980\end{array}$ & $\begin{array}{l}71 \\
58\end{array}$ & $\begin{array}{l}1754 \\
1711\end{array}$ & $\begin{array}{l}35 \\
10\end{array}$ & 1645 & 15 & 1469 & 20 & 1436 & 0 & 1421 & 10 & 1208 & 95 & $\begin{array}{l}1824 \\
1932 \\
3646\end{array}$ & $\begin{array}{l}72 \\
97\end{array}$ & $\begin{array}{l}K \\
K \\
M\end{array}$ \\
\hline 650 & 20 & 559 & 10 & 334 & 25 & -52 & 20 & -136 & 77 & -434 & 15 & -485 & 60 & -726 & 40 & & & -769 & 10 & -958 & 75 & 3949 & & M \\
\hline $\begin{array}{l}712 \\
630\end{array}$ & $\begin{array}{l}20 \\
50\end{array}$ & 594 & 0 & 357 & 15 & -38 & 20 & -128 & 80 & -408 & 25 & -480 & 25 & -703 & 20 & & & -733 & 5 & -958 & 55 & $\begin{array}{l}3907 \\
2231\end{array}$ & $\begin{array}{l}95 \\
71\end{array}$ & $\begin{array}{l}M \\
K\end{array}$ \\
\hline $\begin{array}{l}928 \\
585\end{array}$ & $\begin{array}{l}50 \\
30\end{array}$ & 782 & 15 & 568 & 20 & 213 & 30 & $\begin{array}{r}1075 \\
85\end{array}$ & $\begin{array}{l}100 \\
104\end{array}$ & $\begin{array}{r}826 \\
-161\end{array}$ & $\begin{array}{l}45 \\
75\end{array}$ & $\begin{array}{r}755 \\
-237\end{array}$ & 15 & -357 & 55 & -427 & 0 & & & & & $\begin{array}{l}2903 \\
2008\end{array}$ & & $\begin{array}{l}\mathrm{J} \\
\mathrm{K}\end{array}$ \\
\hline $\begin{array}{r}200 \\
106 \\
31 \\
-397 \\
-424\end{array}$ & $\begin{array}{l}20 \\
15 \\
20 \\
20 \\
30\end{array}$ & $\begin{array}{r}41 \\
-11 \\
-99 \\
-530 \\
-560\end{array}$ & $\begin{array}{l}0 \\
0 \\
0 \\
0 \\
0\end{array}$ & $\begin{array}{l}-179 \\
-282 \\
-344 \\
-767 \\
-776\end{array}$ & $\begin{array}{l}5 \\
0 \\
0 \\
0 \\
0\end{array}$ & $\begin{array}{r}-551 \\
-647 \\
-680 \\
-1068 \\
-1095\end{array}$ & $\begin{array}{r}10 \\
10 \\
5 \\
15 \\
10\end{array}$ & $\begin{array}{r}-584 \\
-682 \\
-728 \\
-1124 \\
-1134\end{array}$ & $\begin{array}{r}130 \\
122 \\
130 \\
96 \\
85\end{array}$ & $\begin{array}{r}-836 \\
-937 \\
-1004 \\
-1373 \\
-1360\end{array}$ & $\begin{array}{l}25 \\
30 \\
55 \\
40 \\
35\end{array}$ & $\begin{array}{r}-895 \\
-997 \\
-1085 \\
-1455 \\
-1427\end{array}$ & $\begin{array}{l}45 \\
20 \\
10 \\
50 \\
20\end{array}$ & $\begin{array}{l}-1030 \\
-1131 \\
-1174 \\
-1597 \\
-1563\end{array}$ & $\begin{array}{l}20 \\
25 \\
30 \\
65 \\
45\end{array}$ & & & $\begin{array}{l}-1067 \\
-1187 \\
-1233 \\
-1668 \\
-1654\end{array}$ & $\begin{array}{l}10 \\
20 \\
15 \\
15 \\
20\end{array}$ & $\begin{array}{l}-1504 \\
-1617 \\
-1684 \\
-2177 \\
-2197\end{array}$ & $\begin{array}{r}80 \\
115 \\
95 \\
95 \\
75\end{array}$ & $\begin{array}{l}6084 \\
5237 \\
5503 \\
7720 \\
5542\end{array}$ & $\begin{array}{l}135 \\
124 \\
134 \\
157 \\
126\end{array}$ & $\begin{array}{l}M \\
M \\
M \\
T \\
T\end{array}$ \\
\hline $\begin{array}{l}-380 \\
-372 \\
-397 \\
-290\end{array}$ & $\begin{array}{l}30 \\
20 \\
40 \\
30\end{array}$ & $\begin{array}{l}-512 \\
-501 \\
-537 \\
-418\end{array}$ & $\begin{array}{l}0 \\
0 \\
0 \\
0\end{array}$ & $\begin{array}{l}-710 \\
-707 \\
-730 \\
-621\end{array}$ & $\begin{array}{l}0 \\
0 \\
0 \\
0\end{array}$ & $\begin{array}{r}-937 \\
-921 \\
-955 \\
-843 \\
-1754\end{array}$ & $\begin{array}{l}20 \\
30 \\
35 \\
20 \\
50\end{array}$ & $\begin{array}{r}-990 \\
-1000 \\
-1029 \\
-908 \\
-1804\end{array}$ & $\begin{array}{l}75 \\
67 \\
79 \\
87 \\
72\end{array}$ & $\begin{array}{l}-1246 \\
-1219 \\
-1226 \\
-1124 \\
-2059\end{array}$ & $\begin{array}{l}35 \\
45 \\
30 \\
40 \\
60\end{array}$ & $\begin{array}{l}-1310 \\
-1279 \\
-1282 \\
-1189 \\
-2134\end{array}$ & $\begin{array}{l}70 \\
90 \\
60 \\
40\end{array}$ & $\begin{array}{l}-1500 \\
-1486 \\
-1502 \\
-1385\end{array}$ & $\begin{array}{l}55 \\
50 \\
70 \\
30\end{array}$ & & & $\begin{array}{l}-1587 \\
-1585 \\
-1594 \\
-1459\end{array}$ & $\begin{array}{l}25 \\
35 \\
50 \\
25\end{array}$ & $\begin{array}{l}-2120 \\
-2110 \\
-2143 \\
-2025\end{array}$ & $\begin{array}{r}90 \\
105 \\
90 \\
75\end{array}$ & $\begin{array}{l}7775 \\
9238 \\
6227 \\
4817\end{array}$ & $\begin{array}{l}162 \\
210 \\
158 \\
171\end{array}$ & $\begin{array}{l}T \\
T \\
T \\
1\end{array}$ \\
\hline-1840 & 40 & -1962 & 0 & -2141 & 0 & -2292 & 80 & -2385 & 81 & -2630 & 50 & -2709 & 90 & -2956 & 35 & & & -3042 & 30 & -3591 & 85 & 11699 & 242 & $\mathbf{I}$ \\
\hline-1927 & 40 & -2044 & 0 & -2239 & 0 & -2414 & 45 & -2472 & 79 & -2707 & 50 & -2784 & 160 & -3060 & 40 & & & -3144 & 40 & -3726 & 85 & 11935 & 206 & $T$ \\
\hline-2053 & 40 & -2203 & 0 & -2395 & 0 & -2573 & 35 & -2618 & 75 & -2878 & 35 & -2945 & 70 & -3093 & 60 & & & -3183 & 45 & -3807 & 90 & 12468 & & $\mathbf{T}$ \\
\hline $\begin{array}{r}-2193 \\
1181\end{array}$ & $\begin{array}{l}45 \\
30\end{array}$ & $\begin{array}{r}-2336 \\
-2400 \\
1136\end{array}$ & $\begin{array}{r}0 \\
0 \\
10\end{array}$ & $\begin{array}{r}-2521 \\
-2586 \\
1075\end{array}$ & $\begin{array}{l}0 \\
0 \\
0\end{array}$ & $\begin{array}{r}-2715 \\
-2785 \\
838\end{array}$ & $\begin{array}{l}55 \\
65 \\
12\end{array}$ & $\begin{array}{r}-2777 \\
-2860 \\
796\end{array}$ & $\begin{array}{l}90 \\
75 \\
35\end{array}$ & $\begin{array}{r}-3006 \\
-3083 \\
479\end{array}$ & $\begin{array}{l}60 \\
50 \\
38\end{array}$ & $\begin{array}{r}-3084 \\
-3154 \\
441\end{array}$ & $\begin{array}{l}75 \\
45 \\
70\end{array}$ & $\begin{array}{r}-3286 \\
-3370 \\
-132\end{array}$ & $\begin{array}{l}45 \\
55 \\
10\end{array}$ & -154 & 15 & $\begin{array}{r}-3360 \\
-3470 \\
-313\end{array}$ & $\begin{array}{l}40 \\
25 \\
65\end{array}$ & $\begin{array}{r}-4000 \\
-4045 \\
-455\end{array}$ & $\begin{array}{l}90 \\
85 \\
20\end{array}$ & $\begin{array}{r}11071 \\
13217 \\
5081\end{array}$ & $\begin{array}{r}214 \\
75\end{array}$ & $\begin{array}{l}T \\
Y \\
M\end{array}$ \\
\hline $\begin{array}{l}2549 \\
2605 \\
3309 \\
2831 \\
2816\end{array}$ & $\begin{array}{l}32 \\
27 \\
22 \\
20 \\
19\end{array}$ & $\begin{array}{l}2504 \\
2569 \\
3279 \\
2802 \\
2793\end{array}$ & $\begin{array}{r}10 \\
10 \\
5 \\
5 \\
5\end{array}$ & $\begin{array}{l}2436 \\
2516 \\
3232 \\
2735 \\
2722\end{array}$ & $\begin{array}{r}0 \\
0 \\
15 \\
15 \\
32\end{array}$ & $\begin{array}{l}2157 \\
2251 \\
2945 \\
2472 \\
2445\end{array}$ & $\begin{array}{l}15 \\
20 \\
30 \\
30 \\
51\end{array}$ & $\begin{array}{l}2108 \\
2186 \\
2849 \\
2302 \\
2357\end{array}$ & $\begin{array}{l}35 \\
50 \\
35 \\
40 \\
45\end{array}$ & $\begin{array}{l}1870 \\
1887 \\
2535 \\
2060 \\
2070\end{array}$ & $\begin{array}{l}30 \\
25 \\
26 \\
28 \\
20\end{array}$ & $\begin{array}{l}1819 \\
1844 \\
2504 \\
2038 \\
2032\end{array}$ & $\begin{array}{l}72 \\
50 \\
25 \\
85 \\
95\end{array}$ & $\begin{array}{l}1294 \\
1361 \\
2078 \\
1590 \\
1623\end{array}$ & $\begin{array}{l}24 \\
42 \\
20 \\
20 \\
25\end{array}$ & $\begin{array}{l}1270 \\
1319 \\
2039 \\
1570 \\
1586\end{array}$ & $\begin{array}{l}10 \\
10 \\
10 \\
10 \\
15\end{array}$ & $\begin{array}{l}1182 \\
1214 \\
1943 \\
1480 \\
1498\end{array}$ & $\begin{array}{l}60 \\
50 \\
40 \\
40 \\
35\end{array}$ & $\begin{array}{l}1057 \\
1112 \\
1841 \\
1384 \\
1392\end{array}$ & $\begin{array}{r}10 \\
20 \\
0 \\
15 \\
20\end{array}$ & $\begin{array}{l}3372 \\
3250 \\
2112 \\
2710 \\
2715\end{array}$ & $\begin{array}{l}95 \\
90 \\
73 \\
84 \\
78\end{array}$ & $\begin{array}{l}M \\
M \\
M \\
M \\
M\end{array}$ \\
\hline
\end{tabular}


TABLE 2. - SELECTED geOLOGIC DATA -- CONTINUED

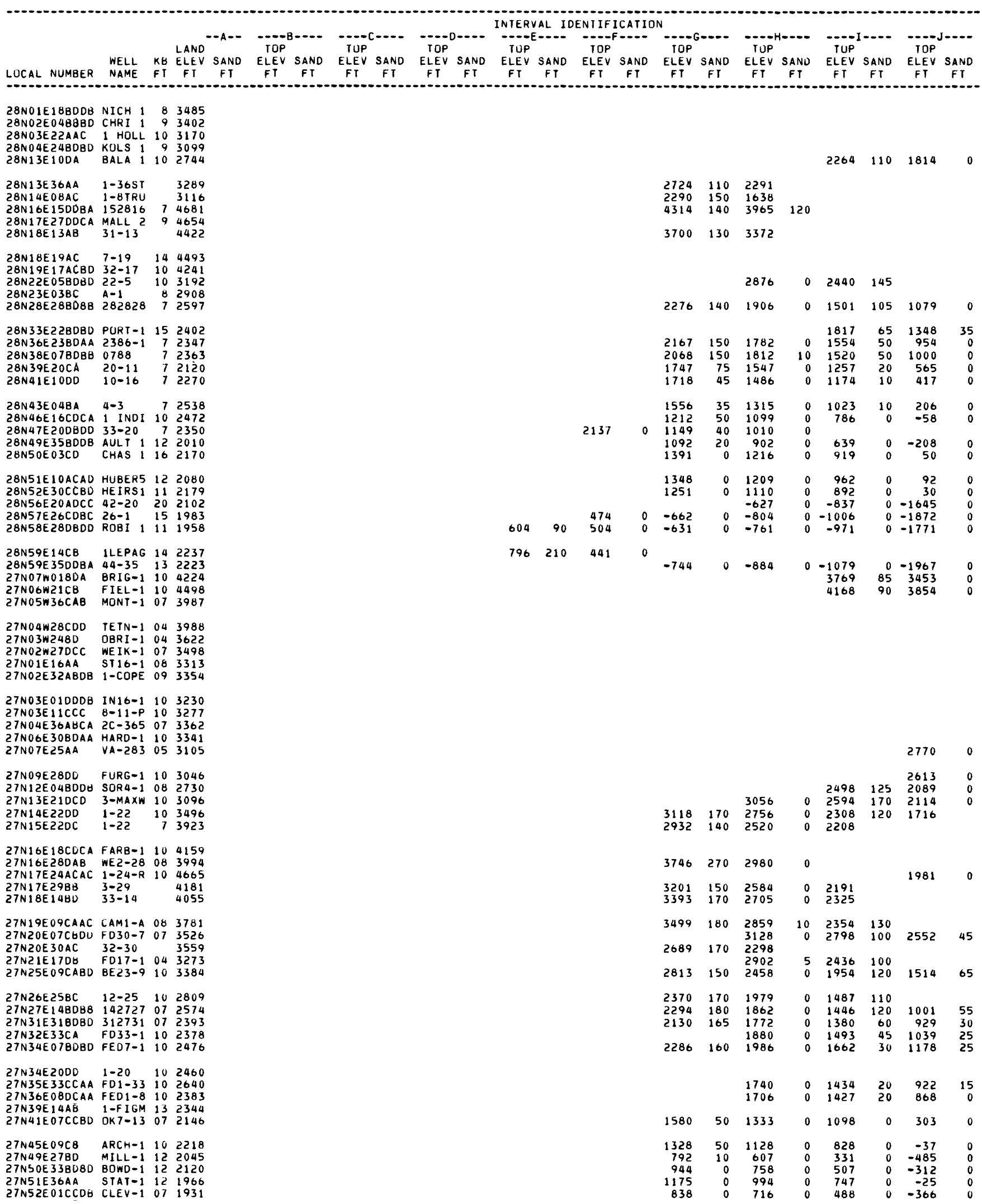




\begin{tabular}{|c|c|c|c|c|c|c|c|c|c|c|c|c|c|c|c|c|c|c|c|c|c|c|c|c|}
\hline $\begin{array}{c}----K \\
\text { TOP } \\
\text { ELEV } \\
\text { FT }\end{array}$ & $\begin{array}{l}\text { SAND } \\
\text { FT }\end{array}$ & $\begin{array}{l}-\because--L \\
\text { TOP } \\
\text { ELEV } \\
\text { FT }\end{array}$ & $\begin{array}{l}\text { SAND } \\
\text { FI }\end{array}$ & \begin{tabular}{l} 
TOP \\
ELEV \\
\hdashline$--L$ \\
\end{tabular} & $\begin{array}{l}\text { SAND } \\
\text { FT }\end{array}$ & $\begin{array}{l}\text { FU } \\
\text { TUP } \\
\text { FLV }\end{array}$ & $\begin{array}{l}\text { SAND } \\
\text { FT }\end{array}$ & $\begin{array}{l}\text { TOP } \\
\text { ELEV } \\
F I\end{array}$ & $\begin{array}{l}\text { INTERV } \\
\text { SAND } \\
\text { FT }\end{array}$ & $\begin{array}{l}A L \text { IOE } \\
\text { TOP } \\
\text { ELEV } \\
\text { FT }\end{array}$ & $\begin{array}{l}\text { SAND } \\
\text { FT }\end{array}$ & $\begin{array}{l}\text { ICATION } \\
\text { TUP } \\
\text { ELEV } \\
\text { FT }\end{array}$ & $\begin{array}{l}\text { SAND } \\
\text { FI }\end{array}$ & $\begin{array}{l}\text { TUP } \\
\text { ELEV } \\
\text { FI }\end{array}$ & $\begin{array}{l}\text { SAND } \\
\text { FT }\end{array}$ & $\begin{array}{l}\text { IUP } \\
\text { ELEV S } \\
\text { FT }\end{array}$ & $\begin{array}{l}\text { SAND } \\
\text { FT }\end{array}$ & $\begin{array}{l}\text { TUP } \\
\text { ELEV } \\
\text { FT }\end{array}$ & $\begin{array}{l}\text { SAND } \\
\text { FI }\end{array}$ & $\begin{array}{l}\text { IUP } \\
\text { ELEV } \\
\text { FT }\end{array}$ & $\begin{array}{l}\text { T }=-- \\
\text { SANO } \\
\text { FT }\end{array}$ & $\begin{array}{l}\text { WELL } \\
\text { DEPTH }\end{array}$ & $\begin{array}{c}\text { TEMP } \\
-\quad F\end{array}$ & $\begin{array}{l}6 \\
\mathrm{E}\end{array}$ \\
\hline $\begin{array}{l}2944 \\
2785 \\
2558 \\
2375 \\
1204\end{array}$ & $\begin{array}{l}19 \\
13 \\
15 \\
12 \\
12\end{array}$ & $\begin{array}{l}2906 \\
2749 \\
2514 \\
2341 \\
1158\end{array}$ & $\begin{array}{r}0 \\
0 \\
0 \\
0 \\
10\end{array}$ & $\begin{array}{l}2861 \\
2699 \\
2474 \\
2296 \\
1029\end{array}$ & $\begin{array}{l}55 \\
90 \\
80 \\
60 \\
55\end{array}$ & $\begin{array}{r}2557 \\
2413 \\
2147 \\
1989 \\
082\end{array}$ & $\begin{array}{l}46 \\
32 \\
38 \\
65 \\
45\end{array}$ & $\begin{array}{r}2395 \\
2213 \\
1954 \\
1802 \\
516\end{array}$ & $\begin{array}{l}75 \\
25 \\
50 \\
55 \\
40\end{array}$ & $\begin{array}{l}2105 \\
1933 \\
1670 \\
258\end{array}$ & $\begin{array}{l}15 \\
28 \\
16 \\
20\end{array}$ & $\begin{array}{l}2009 \\
1895 \\
1642 \\
223\end{array}$ & $\begin{array}{l}40 \\
35 \\
25\end{array}$ & $\begin{array}{r}1725 \\
1514 \\
1298 \\
-15\end{array}$ & $\begin{array}{l}14 \\
18 \\
29 \\
69\end{array}$ & $\begin{array}{r}1698 \\
1491 \\
1269 \\
-84\end{array}$ & $\begin{array}{l}0 \\
0 \\
0\end{array}$ & $\begin{array}{l}1603 \\
1431 \\
1201 \\
-131\end{array}$ & $\begin{array}{l}45 \\
58 \\
40 \\
\\
55\end{array}$ & $\begin{array}{l}1522 \\
1120 \\
-252\end{array}$ & $\begin{array}{l}10 \\
10 \\
20\end{array}$ & $\begin{array}{l}2090 \\
2127 \\
2170 \\
1650 \\
3238\end{array}$ & $\begin{array}{l}85 \\
80\end{array}$ & $\begin{array}{l}M \\
M \\
M \\
K \\
M\end{array}$ \\
\hline & & & & & & & & & & & & & & & & & & & & & & $\begin{array}{l}1314 \\
3372\end{array}$ & $2 \quad 82$ & $\begin{array}{l}k \\
k\end{array}$ \\
\hline 2688 & 25 & 2598 & 15 & & & & & 1427 & 95 & 1067 & 64 & 1003 & & & & & & & & & & $\begin{array}{l}1883 \\
1391\end{array}$ & 70 & $\begin{array}{l}K \\
k\end{array}$ \\
\hline 534 & 40 & & & & & 289 & 10 & 266 & 90 & -46 & 40 & -116 & & -276 & 33 & -309 & & -324 & 40 & -529 & & 2185 & & $k$ \\
\hline $\begin{array}{l}765 \\
437\end{array}$ & $\begin{array}{l}20 \\
15\end{array}$ & 644 & 0 & 427 & 15 & 68 & 25 & -35 & 111 & -314 & 50 & -402 & 30 & -487 & 50 & & & -563 & 30 & -687 & 60 & $\begin{array}{l}2515 \\
2131 \\
2195\end{array}$ & 96 & $\begin{array}{l}M \\
K \\
K\end{array}$ \\
\hline $\begin{array}{l}97 \\
-5\end{array}$ & $\begin{array}{l}25 \\
20\end{array}$ & $\begin{array}{r}-15 \\
-175\end{array}$ & $\begin{array}{l}0 \\
0\end{array}$ & $\begin{array}{l}-246 \\
-411\end{array}$ & $\begin{array}{r}10 \\
5\end{array}$ & $\begin{array}{l}-646 \\
-782\end{array}$ & $\begin{array}{l}25 \\
10\end{array}$ & $\begin{array}{l}-678 \\
-806\end{array}$ & $\begin{array}{l}102 \\
130\end{array}$ & $\begin{array}{r}-893 \\
-1067\end{array}$ & $\begin{array}{l}55 \\
50\end{array}$ & $\begin{array}{r}-973 \\
-1143\end{array}$ & $\begin{array}{l}55 \\
15\end{array}$ & $\begin{array}{l}-1140 \\
-1287\end{array}$ & $\begin{array}{l}55 \\
40\end{array}$ & & & $\begin{array}{l}-1209 \\
-1363\end{array}$ & $\begin{array}{l}40 \\
15\end{array}$ & $\begin{array}{l}-1614 \\
-1773\end{array}$ & $\begin{array}{r}100 \\
80\end{array}$ & $\begin{array}{l}4582 \\
4850\end{array}$ & $\begin{array}{l}145 \\
126\end{array}$ & $M$ \\
\hline $\begin{array}{l}-175 \\
-448\end{array}$ & $\begin{array}{l}20 \\
30\end{array}$ & $\begin{array}{l}-345 \\
-582\end{array}$ & $\begin{array}{l}0 \\
0\end{array}$ & $\begin{array}{l}-569 \\
-824\end{array}$ & $\begin{array}{l}0 \\
0\end{array}$ & $\begin{array}{r}-935 \\
-1126\end{array}$ & $\begin{array}{r}5 \\
10\end{array}$ & $\begin{array}{r}-960 \\
-1164\end{array}$ & $\begin{array}{r}105 \\
91\end{array}$ & $\begin{array}{l}-1210 \\
-1413\end{array}$ & $\begin{array}{l}55 \\
45\end{array}$ & $\begin{array}{l}-1307 \\
-1473\end{array}$ & $\begin{array}{l}25 \\
30\end{array}$ & $\begin{array}{l}-1446 \\
-1608\end{array}$ & $\begin{array}{l}45 \\
80\end{array}$ & & & $\begin{array}{l}-1521 \\
-1711\end{array}$ & $\begin{array}{l}15 \\
20\end{array}$ & $\begin{array}{l}-1949 \\
-2206\end{array}$ & $\begin{array}{r}85 \\
100\end{array}$ & $\begin{array}{l}5510 \\
7411 \\
2219\end{array}$ & $\begin{array}{l}139 \\
160\end{array}$ & $\begin{array}{l}T \\
T \\
K\end{array}$ \\
\hline $\begin{array}{l}-509 \\
-290\end{array}$ & $\begin{array}{l}25 \\
40\end{array}$ & $\begin{array}{l}-668 \\
-414\end{array}$ & $\begin{array}{l}0 \\
0\end{array}$ & $\begin{array}{l}-906 \\
-648\end{array}$ & $\begin{array}{l}0 \\
0\end{array}$ & $\begin{array}{r}-1143 \\
-877\end{array}$ & $\begin{array}{l}20 \\
20\end{array}$ & $\begin{array}{r}-1202 \\
-944\end{array}$ & $\begin{array}{l}92 \\
82\end{array}$ & $\begin{array}{l}-1441 \\
-1176\end{array}$ & $\begin{array}{l}30 \\
55\end{array}$ & $\begin{array}{l}-1498 \\
-1275\end{array}$ & $\begin{array}{l}80 \\
30\end{array}$ & $\begin{array}{l}-1698 \\
-1392\end{array}$ & $\begin{array}{l}50 \\
45\end{array}$ & & & $\begin{array}{l}-1768 \\
-1463\end{array}$ & $\begin{array}{l}35 \\
30\end{array}$ & $\begin{array}{l}-2295 \\
-2047\end{array}$ & $\begin{array}{l}125 \\
130\end{array}$ & $\begin{array}{l}7466 \\
7645\end{array}$ & $\begin{array}{l}164 \\
192\end{array}$ & T \\
\hline $\begin{array}{r}-246 \\
-329 \\
-2029 \\
-2269 \\
-2179\end{array}$ & $\begin{array}{l}35 \\
45 \\
40 \\
35 \\
45\end{array}$ & $\begin{array}{r}-300 \\
-452 \\
-2137 \\
-2440 \\
-2351\end{array}$ & $\begin{array}{l}0 \\
0 \\
0 \\
0 \\
0\end{array}$ & $\begin{array}{r}-596 \\
-694 \\
-2352 \\
-2625 \\
-2535\end{array}$ & $\begin{array}{l}0 \\
0 \\
0 \\
0 \\
0\end{array}$ & $\begin{array}{r}-828 \\
-898 \\
-2527 \\
-2777 \\
-2720\end{array}$ & $\begin{array}{l}25 \\
30 \\
35 \\
20 \\
50\end{array}$ & $\begin{array}{r}-862 \\
-950 \\
-2566 \\
-2835 \\
-2771\end{array}$ & $\begin{array}{l}79 \\
78 \\
78 \\
90 \\
72\end{array}$ & $\begin{array}{l}-1095 \\
-1197 \\
-2804 \\
=3097 \\
-3009\end{array}$ & $\begin{array}{l}70 \\
45 \\
35 \\
45 \\
45\end{array}$ & $\begin{array}{l}-1209 \\
-1289 \\
-2877 \\
-3172 \\
-3082\end{array}$ & $\begin{array}{r}75 \\
85 \\
160 \\
90 \\
100\end{array}$ & $\begin{array}{l}-1377 \\
-1485 \\
-3100 \\
-3372 \\
-3276\end{array}$ & $\begin{array}{r}40 \\
50 \\
110 \\
75 \\
50\end{array}$ & & & $\begin{array}{l}-1477 \\
-1575 \\
-3240 \\
-3472 \\
-3373\end{array}$ & $\begin{array}{l}30 \\
25 \\
45 \\
50 \\
60\end{array}$ & $\begin{array}{l}-1983 \\
-2091 \\
-3819 \\
-4060 \\
-3933\end{array}$ & $\begin{array}{r}110 \\
95 \\
100 \\
105\end{array}$ & $\begin{array}{r}7295 \\
6051 \\
12065 \\
12467 \\
6183\end{array}$ & $\begin{array}{l}252 \\
183 \\
230 \\
145\end{array}$ & $\begin{array}{l}\mathrm{T} \\
\mathrm{T} \\
\mathrm{T} \\
\mathrm{I} \\
\mathrm{J}\end{array}$ \\
\hline $\begin{array}{r}-2379 \\
2494 \\
2925 \\
3386\end{array}$ & $\begin{array}{l}30 \\
22 \\
13 \\
32\end{array}$ & $\begin{array}{r}-2561 \\
2464 \\
2903 \\
3354\end{array}$ & $\begin{array}{r}0 \\
0 \\
0 \\
10\end{array}$ & $\begin{array}{r}-2747 \\
2424 \\
2870 \\
3313\end{array}$ & $\begin{array}{l}0 \\
30 \\
28 \\
20\end{array}$ & $\begin{array}{r}-2924 \\
2106 \\
2622 \\
3040\end{array}$ & $\begin{array}{l}60 \\
28 \\
31 \\
44\end{array}$ & $\begin{array}{r}-3007 \\
1972 \\
2490 \\
2908\end{array}$ & $\begin{array}{l}80 \\
30 \\
50 \\
30\end{array}$ & $\begin{array}{r}-3228 \\
1784 \\
2218 \\
2624\end{array}$ & $\begin{array}{l}60 \\
50 \\
20 \\
30\end{array}$ & $\begin{array}{r}-3321 \\
1734 \\
2185 \\
2573\end{array}$ & $\begin{array}{l}55 \\
38 \\
58 \\
98\end{array}$ & $\begin{array}{r}-3494 \\
1281 \\
1711 \\
2145\end{array}$ & $\begin{array}{l}60 \\
22 \\
16 \\
15\end{array}$ & $\begin{array}{l}1259 \\
1695 \\
2130\end{array}$ & $\begin{array}{l}20 \\
18 \\
15\end{array}$ & $\begin{array}{r}-3587 \\
1126 \\
1566 \\
2009\end{array}$ & $\begin{array}{l}35 \\
52 \\
38 \\
33\end{array}$ & $\begin{array}{r}-4160 \\
1022 \\
1444 \\
1897\end{array}$ & $\begin{array}{l}90 \\
12 \\
12 \\
12\end{array}$ & $\begin{array}{r}13137 \\
3371 \\
3196 \\
2167\end{array}$ & $\begin{array}{r}242 \\
97 \\
75\end{array}$ & $\begin{array}{l}T \\
M \\
M \\
M\end{array}$ \\
\hline $\begin{array}{l}3375 \\
3283 \\
3223 \\
2951 \\
2862\end{array}$ & $\begin{array}{l}28 \\
20 \\
23 \\
14 \\
12\end{array}$ & $\begin{array}{l}3347 \\
3253 \\
3192 \\
2916 \\
2846\end{array}$ & $\begin{array}{r}10 \\
0 \\
0 \\
10 \\
0\end{array}$ & $\begin{array}{l}3311 \\
3194 \\
3136 \\
2830 \\
2771\end{array}$ & $\begin{array}{l}20 \\
20 \\
35 \\
78 \\
50\end{array}$ & $\begin{array}{l}3033 \\
2900 \\
2816 \\
2538 \\
2497\end{array}$ & $\begin{array}{l}36 \\
25 \\
28 \\
35 \\
27\end{array}$ & $\begin{array}{l}2905 \\
2822 \\
2762 \\
2408 \\
2356\end{array}$ & $\begin{array}{l}64 \\
86 \\
68 \\
80 \\
50\end{array}$ & $\begin{array}{l}2605 \\
2499 \\
2425 \\
2112 \\
2055\end{array}$ & $\begin{array}{l}35 \\
30 \\
37 \\
28 \\
25\end{array}$ & $\begin{array}{l}2553 \\
2466 \\
2388 \\
2064 \\
1983\end{array}$ & $\begin{array}{r}70 \\
105 \\
40 \\
30 \\
32\end{array}$ & $\begin{array}{l}2106 \\
2048 \\
2060 \\
1731 \\
1706\end{array}$ & $\begin{array}{l}12 \\
22 \\
25 \\
10 \\
12\end{array}$ & $\begin{array}{l}2094 \\
2026 \\
2035 \\
1709 \\
1677\end{array}$ & $\begin{array}{r}15 \\
18 \\
15 \\
21 \\
0\end{array}$ & $\begin{array}{l}1985 \\
1931 \\
1897 \\
1609 \\
1594\end{array}$ & $\begin{array}{l}35 \\
50 \\
62 \\
56 \\
40\end{array}$ & $\begin{array}{l}1908 \\
1845 \\
1823 \\
1510 \\
1491\end{array}$ & $\begin{array}{l}10 \\
10 \\
12 \\
15 \\
15\end{array}$ & $\begin{array}{l}2168 \\
1856 \\
1814 \\
1912 \\
1987\end{array}$ & $\begin{array}{l}73 \\
79 \\
74\end{array}$ & $\begin{array}{l}M \\
M \\
M \\
M \\
M\end{array}$ \\
\hline $\begin{array}{l}2630 \\
2703 \\
2506 \\
2306 \\
2016\end{array}$ & $\begin{array}{l}10 \\
18 \\
17 \\
12 \\
17\end{array}$ & $\begin{array}{l}2620 \\
2685 \\
2473 \\
2274 \\
1984\end{array}$ & $\begin{array}{l}0 \\
0 \\
0 \\
0 \\
0\end{array}$ & $\begin{array}{l}2551 \\
2595 \\
2412 \\
2199 \\
1911\end{array}$ & $\begin{array}{l}80 \\
44 \\
75 \\
65 \\
56\end{array}$ & $\begin{array}{l}2248 \\
2291 \\
2114 \\
1906 \\
1577\end{array}$ & $\begin{array}{l}30 \\
30 \\
45 \\
45 \\
45\end{array}$ & $\begin{array}{l}2067 \\
2149 \\
1925 \\
1746 \\
1443\end{array}$ & $\begin{array}{l}50 \\
65 \\
35 \\
55 \\
83\end{array}$ & $\begin{array}{l}1770 \\
1857 \\
1634 \\
1436 \\
1125\end{array}$ & $\begin{array}{l}28 \\
25 \\
30 \\
20 \\
25\end{array}$ & $\begin{array}{l}1700 \\
1825 \\
1502 \\
1378 \\
1067\end{array}$ & $\begin{array}{l}25 \\
30 \\
50 \\
30 \\
30\end{array}$ & $\begin{array}{r}1422 \\
1514 \\
1292 \\
1101 \\
808\end{array}$ & $\begin{array}{l}15 \\
19 \\
43 \\
39 \\
22\end{array}$ & $\begin{array}{l}1375 \\
1495 \\
1246 \\
1062 \\
773\end{array}$ & $\begin{array}{l}0 \\
10 \\
15 \\
12 \\
10\end{array}$ & $\begin{array}{r}1323 \\
1402 \\
1177 \\
994 \\
688\end{array}$ & $\begin{array}{l}40 \\
55 \\
37 \\
54 \\
50\end{array}$ & $\begin{array}{r}1232 \\
1273 \\
1092 \\
875 \\
594\end{array}$ & $\begin{array}{l}0 \\
0 \\
0 \\
0 \\
0\end{array}$ & $\begin{array}{l}2135 \\
4202 \\
2353 \\
2510 \\
2825\end{array}$ & $\begin{array}{l}103 \\
77 \\
85\end{array}$ & $\begin{array}{l}M \\
M \\
M \\
M \\
M\end{array}$ \\
\hline $\begin{array}{l}1913 \\
1428 \\
1503\end{array}$ & $\begin{array}{l}10 \\
14 \\
12\end{array}$ & $\begin{array}{l}1878 \\
1383 \\
1476\end{array}$ & $\begin{array}{r}10 \\
15 \\
0\end{array}$ & $\begin{array}{l}1806 \\
1258 \\
1338\end{array}$ & $\begin{array}{l}40 \\
55 \\
45\end{array}$ & $\begin{array}{r}1426 \\
870 \\
941\end{array}$ & $\begin{array}{l}44 \\
45 \\
42\end{array}$ & $\begin{array}{r}1260 \\
708 \\
777\end{array}$ & $\begin{array}{l}33 \\
25 \\
25\end{array}$ & $\begin{array}{l}995 \\
463 \\
529\end{array}$ & $\begin{array}{l}25 \\
20 \\
20\end{array}$ & $\begin{array}{l}931 \\
426 \\
456\end{array}$ & $\begin{array}{r}31 \\
30 \\
8\end{array}$ & $\begin{array}{l}692 \\
135 \\
448\end{array}$ & $\begin{array}{l}36 \\
15 \\
40\end{array}$ & $\begin{array}{l}656 \\
103 \\
206\end{array}$ & $\begin{array}{l}10 \\
10 \\
10\end{array}$ & $\begin{array}{r}577 \\
45 \\
132\end{array}$ & $\begin{array}{l}55 \\
70 \\
65\end{array}$ & $\begin{array}{r}486 \\
-86 \\
16\end{array}$ & $\begin{array}{r}10 \\
22 \\
5\end{array}$ & $\begin{array}{l}2623 \\
2957 \\
3760\end{array}$ & $\begin{array}{l}80 \\
82 \\
8 B\end{array}$ & $\begin{array}{l}M \\
M \\
M\end{array}$ \\
\hline 1274 & 22 & 1235 & 15 & 1066 & 40 & 724 & 58 & 544 & 50 & 266 & 20 & 229 & 35 & 17 & 35 & -44 & 15 & -134 & so & -252 & 20 & $\begin{array}{l}4658 \\
2035\end{array}$ & $\begin{array}{r}120 \\
\quad 75\end{array}$ & $M$ \\
\hline 1210 & 21 & 1174 & 0 & 973 & 33 & 618 & 75 & 457 & 30 & 173 & 20 & 120 & 10 & -63 & 40 & -113 & 40 & -238 & 29 & -321 & 0 & 5277 & 119 & M \\
\hline 1991 & 15 & 1924 & 10 & 1738 & 30 & 1388 & 85 & & & & & & & & & & & & & & & $\begin{array}{l}1990 \\
2338\end{array}$ & $\begin{array}{l}90 \\
76\end{array}$ & $\begin{array}{l}k \\
k\end{array}$ \\
\hline 976 & 15 & 906 & 0 & 806 & 10 & 244 & 15 & 94 & 68 & -114 & 10 & -170 & 0 & -337 & 40 & -384 & 0 & & & & & $\begin{array}{l}1381 \\
3915\end{array}$ & $\begin{array}{l}69 \\
88\end{array}$ & $\begin{array}{l}k \\
j\end{array}$ \\
\hline $\begin{array}{l}481 \\
378 \\
467\end{array}$ & $\begin{array}{l}30 \\
30 \\
10\end{array}$ & $\begin{array}{l}301 \\
376\end{array}$ & $\begin{array}{r}10 \\
0\end{array}$ & & & & & -283 & 75 & -489 & 68 & -557 & & -713 & 36 & -749 & & -821 & 55 & -1031 & & $\begin{array}{l}2300 \\
2262 \\
2210 \\
2004\end{array}$ & $\begin{array}{l}72 \\
75\end{array}$ & $\begin{array}{l}K \\
k \\
K \\
K\end{array}$ \\
\hline $\begin{array}{l}368 \\
333 \\
-82\end{array}$ & $\begin{array}{l}20 \\
20 \\
20\end{array}$ & $\begin{array}{r}238 \\
211 \\
-237\end{array}$ & $\begin{array}{l}0 \\
0\end{array}$ & $\begin{array}{r}-8 \\
-30 \\
-474\end{array}$ & $\begin{array}{r}10 \\
5\end{array}$ & $\begin{array}{l}-429 \\
-447 \\
-805 \\
-863\end{array}$ & $\begin{array}{r}20 \\
20 \\
5 \\
15\end{array}$ & $\begin{array}{l}-353 \\
-469 \\
-486 \\
-817 \\
-887\end{array}$ & $\begin{array}{l}100 \\
102 \\
105 \\
100 \\
1113\end{array}$ & $\begin{array}{r}-581 \\
-692 \\
-716 \\
-1057 \\
-1137\end{array}$ & $\begin{array}{l}80 \\
85 \\
95 \\
85 \\
40\end{array}$ & $\begin{array}{r}-675 \\
-805 \\
-832 \\
-1142 \\
-1210\end{array}$ & $\begin{array}{l}45 \\
30\end{array}$ & $\begin{array}{r}-915 \\
-942 \\
-1368\end{array}$ & $\begin{array}{l}45 \\
55\end{array}$ & & & $\begin{array}{r}-977 \\
-1028 \\
-1427\end{array}$ & $\begin{array}{l}35 \\
20\end{array}$ & $\begin{array}{l}-1350 \\
-1371 \\
-1847\end{array}$ & $\begin{array}{l}70 \\
70\end{array}$ & $\begin{array}{l}6876 \\
6814\end{array}$ & $\begin{array}{l}153 \\
148\end{array}$ & $\begin{array}{l}M \\
M\end{array}$ \\
\hline $\begin{array}{l}-408 \\
-863 \\
-691 \\
-448 \\
-772\end{array}$ & $\begin{array}{l}50 \\
13 \\
40 \\
45 \\
50\end{array}$ & $\begin{array}{l}-557 \\
-964 \\
-767 \\
-534 \\
-892\end{array}$ & $\begin{array}{l}0 \\
0 \\
0 \\
0 \\
0\end{array}$ & $\begin{array}{r}-785 \\
-1148 \\
-1011 \\
-822 \\
-1115\end{array}$ & $\begin{array}{l}0 \\
0 \\
0 \\
0 \\
0\end{array}$ & $\begin{array}{l}-1122 \\
-1473 \\
-1261 \\
-1042 \\
-1332\end{array}$ & $\begin{array}{l}15 \\
20 \\
40 \\
40 \\
35\end{array}$ & $\begin{array}{l}-1148 \\
-1513 \\
-1302 \\
-1097 \\
-1370\end{array}$ & $\begin{array}{l}90 \\
80 \\
91 \\
75 \\
67\end{array}$ & $\begin{array}{l}-1382 \\
-1765 \\
-1551 \\
-1347 \\
-1614\end{array}$ & $\begin{array}{l}80 \\
40 \\
40 \\
30 \\
30\end{array}$ & $\begin{array}{l}-1474 \\
-1853 \\
-1633 \\
-1427 \\
-1688\end{array}$ & $\begin{array}{l}40 \\
30 \\
40 \\
40 \\
75\end{array}$ & $\begin{array}{l}-1618 \\
-2023 \\
-1816 \\
-1620 \\
-1923\end{array}$ & $\begin{array}{l}40 \\
50 \\
60 \\
50 \\
65\end{array}$ & & & $\begin{array}{l}-1670 \\
-2088 \\
-1903 \\
-1703 \\
-2020\end{array}$ & $\begin{array}{l}40 \\
20 \\
20 \\
20 \\
30\end{array}$ & $\begin{array}{l}-2167 \\
-2619 \\
-2440 \\
-2252 \\
-2552\end{array}$ & $\begin{array}{r}115 \\
100 \\
90 \\
110 \\
90\end{array}$ & $\begin{array}{l}5066 \\
9066 \\
6658 \\
5436 \\
6964\end{array}$ & $\begin{array}{l}120 \\
177 \\
160 \\
150 \\
175\end{array}$ & $\begin{array}{l}T \\
\text { T } \\
\text { T } \\
\text { t } \\
\text { T }\end{array}$ \\
\hline
\end{tabular}


TABLE 2. -- SELECTED GEOLOGIC DATA -- CONTINUED

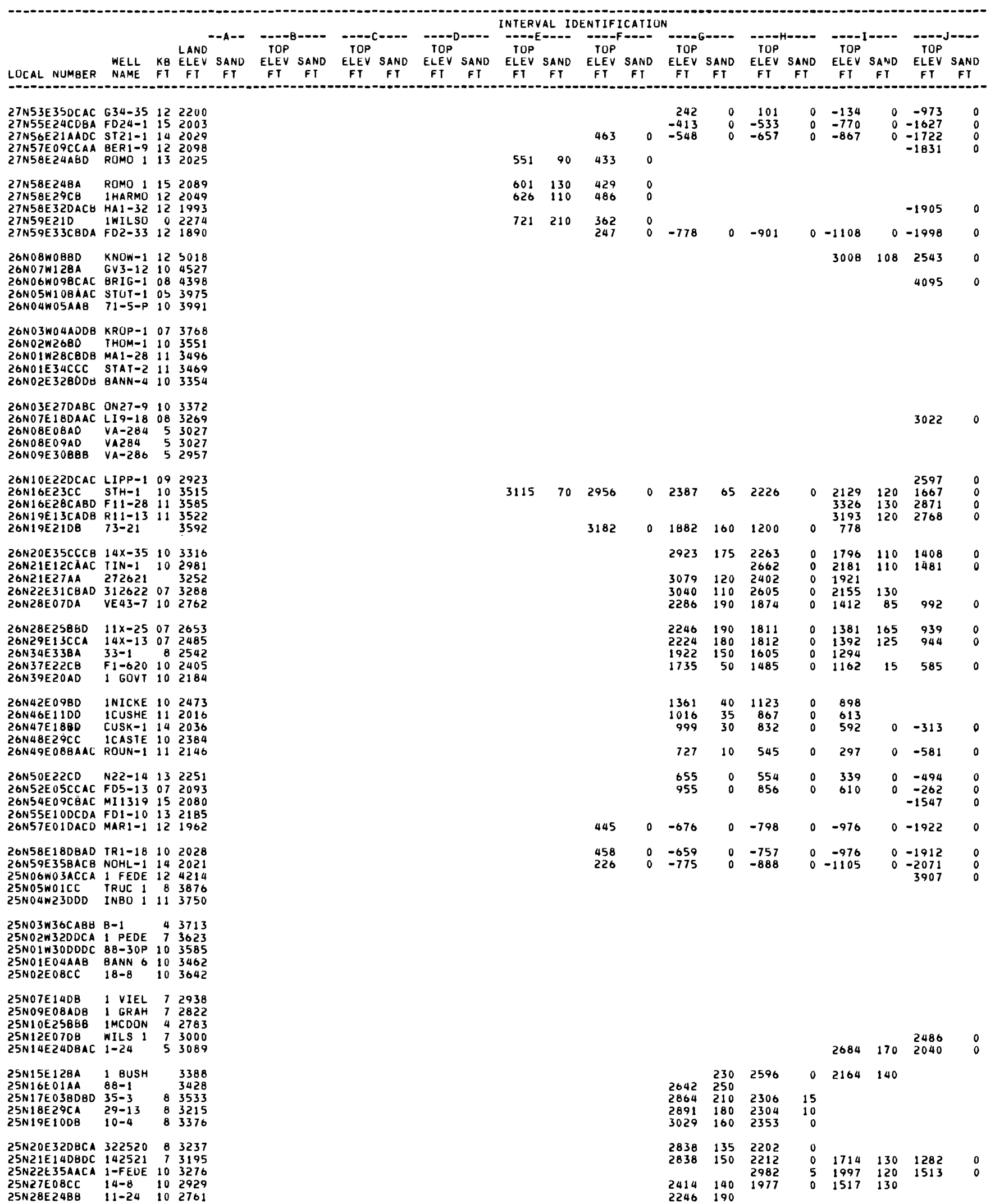




\begin{tabular}{|c|c|c|c|c|c|c|c|c|c|c|c|c|c|c|c|c|c|c|c|c|c|c|c|c|}
\hline $\begin{array}{l}\text { TOP } \\
\text { ELEV }\end{array}$ & $\begin{array}{l}\text { SAND } \\
\text { FT }\end{array}$ & $\begin{array}{l}\text { TOP } \\
\text { ELEV } \\
F T\end{array}$ & $\begin{array}{l}\text { SAND } \\
\text { FI }\end{array}$ & $\begin{array}{c}---D^{-L} \\
\text { TOP } \\
\text { ELEV } \\
F T\end{array}$ & $\begin{array}{l}1--\infty \\
\text { SAND } \\
\text { FT }\end{array}$ & $\begin{array}{l}\text { IOP } \\
\text { ELEV } \\
F T\end{array}$ & $\begin{array}{l}\text { SAND } \\
\text { FT }\end{array}$ & $\begin{array}{l}-E-N \\
\text { TOP } \\
\text { ELEV } \\
\text { FT }\end{array}$ & $\begin{array}{l}\text { SAND } \\
\text { FT }\end{array}$ & $\begin{array}{l}-O-0 \\
\text { IUP } \\
\text { ELEV } \\
F T\end{array}$ & $\begin{array}{l}\text { SAND } \\
\text { FI }\end{array}$ & $\begin{array}{l}-O-P \\
\text { TUP } \\
\text { ELEV } \\
\text { FI }\end{array}$ & $\begin{array}{l}\text { SAND } \\
\text { FT }\end{array}$ & $\begin{array}{l}\text { IUP } \\
\text { ELEV } \\
F I\end{array}$ & $\begin{array}{l}\text { SAND } \\
\text { FI }\end{array}$ & $\begin{array}{l}\text { TOP } \\
\text { ELEV } \\
F I\end{array}$ & $\begin{array}{l}\text { SAND } \\
\text { FT }\end{array}$ & $\begin{array}{l}\text { IOP } \\
\text { ELEV } \\
F T\end{array}$ & $\begin{array}{c}\text { SAND } \\
\text { FT }\end{array}$ & $\begin{array}{l}-D-1 \\
\text { TUP } \\
\text { ELEV }\end{array}$ & $\begin{array}{c}\text { SANO } \\
\text { FT }\end{array}$ & $\begin{array}{l}\text { NELL } \\
\text { DEPTH }\end{array}$ & TEMP & $\begin{array}{l}A \\
G \\
E\end{array}$ \\
\hline $\begin{array}{l}-1389 \\
-2007 \\
-2119 \\
-2230\end{array}$ & $\begin{array}{l}60 \\
65 \\
40 \\
45\end{array}$ & $\begin{array}{l}-1545 \\
-2157 \\
-2271 \\
-2399\end{array}$ & $\begin{array}{l}0 \\
0 \\
0 \\
0\end{array}$ & $\begin{array}{l}-1744 \\
-2355 \\
-2467 \\
-2599\end{array}$ & $\begin{array}{l}0 \\
0 \\
0 \\
0\end{array}$ & $\begin{array}{l}-1943 \\
-2537 \\
-2637 \\
-2755\end{array}$ & $\begin{array}{l}55 \\
35 \\
45 \\
30\end{array}$ & $\begin{array}{l}-2009 \\
-2582 \\
-2693 \\
-2815\end{array}$ & $\begin{array}{l}86 \\
81 \\
75 \\
79\end{array}$ & $\begin{array}{l}-2260 \\
-2828 \\
-2937 \\
-3032\end{array}$ & $\begin{array}{l}35 \\
35 \\
40 \\
40\end{array}$ & $\begin{array}{l}-2327 \\
-2907 \\
-3032 \\
-3119\end{array}$ & $\begin{array}{r}115 \\
120 \\
100 \\
90\end{array}$ & $\begin{array}{l}-2634 \\
-3274 \\
-3292 \\
-3344\end{array}$ & $\begin{array}{l}20 \\
25 \\
55 \\
55\end{array}$ & & & $\begin{array}{l}-2716 \\
-3374 \\
-3386 \\
-3445\end{array}$ & $\begin{array}{l}40 \\
50 \\
75 \\
65\end{array}$ & $\begin{array}{l}-3190 \\
-3847 \\
-3947 \\
-4044\end{array}$ & $\begin{array}{r}100 \\
90 \\
100 \\
110\end{array}$ & $\begin{array}{l}11148 \\
11927 \\
12096 \\
12338\end{array}$ & $\begin{array}{l}238 \\
246 \\
240\end{array}$ & $\begin{array}{l}T \\
1\end{array}$ \\
\hline 2312 & 40 & -2483 & 0 & -2685 & 0 & -2870 & 55 & -2933 & 80 & -3165 & 50 & -3251 & 115 & -3480 & 60 & & & -3570 & 50 & -4134 & 125 & 12638 & & 1 \\
\hline 2415 & 40 & -2592 & 0 & -2799 & 0 & -2977 & 60 & -3058 & 79 & -3287 & 40 & -3354 & 70 & -3546 & 55 & -3648 & 0 & -3658 & 60 & -4208 & 105 & 9217 & 190 & $T$ \\
\hline $\begin{array}{l}1390 \\
3147 \\
3119 \\
3360 \\
3401\end{array}$ & $\begin{array}{l}28 \\
20 \\
21 \\
20 \\
20\end{array}$ & $\begin{array}{l}1350 \\
3117 \\
3093 \\
3330 \\
3369\end{array}$ & $\begin{array}{l}0 \\
0 \\
0 \\
0 \\
0\end{array}$ & $\begin{array}{l}1278 \\
3065 \\
3044 \\
3288 \\
3323\end{array}$ & $\begin{array}{l}30 \\
30 \\
25 \\
25 \\
25\end{array}$ & $\begin{array}{r}928 \\
2792 \\
2773 \\
3037 \\
3049\end{array}$ & $\begin{array}{l}33 \\
25 \\
25 \\
43 \\
45\end{array}$ & $\begin{array}{r}850 \\
2661 \\
2652 \\
2875 \\
2905\end{array}$ & $\begin{array}{l}65 \\
24 \\
40 \\
27 \\
35\end{array}$ & $\begin{array}{l}517 \\
2389 \\
2376 \\
2590 \\
2623\end{array}$ & $\begin{array}{l}27 \\
30 \\
22 \\
38 \\
40\end{array}$ & $\begin{array}{r}465 \\
2345 \\
2333 \\
2547 \\
2563\end{array}$ & $\begin{array}{l}30 \\
25 \\
60 \\
78 \\
20\end{array}$ & $\begin{array}{l}-43 \\
1857 \\
1874 \\
2100 \\
2153\end{array}$ & $\begin{array}{r}26 \\
10 \\
15 \\
8 \\
10\end{array}$ & $\begin{array}{l}-69 \\
1847 \\
1846 \\
2092 \\
2138\end{array}$ & $\begin{array}{l}20 \\
15 \\
20 \\
15 \\
15\end{array}$ & $\begin{array}{l}-220 \\
1677 \\
1716 \\
1965 \\
2021\end{array}$ & $\begin{array}{l}40 \\
20 \\
55 \\
59 \\
65\end{array}$ & $\begin{array}{l}-327 \\
1588 \\
1610 \\
1847 \\
1927\end{array}$ & $\begin{array}{r}17 \\
0 \\
10 \\
10 \\
10\end{array}$ & $\begin{array}{l}7841 \\
3323 \\
2900 \\
2178 \\
2097\end{array}$ & $\begin{array}{r}128 \\
79 \\
86 \\
77 \\
98\end{array}$ & $\begin{array}{l}M \\
M \\
M \\
M\end{array}$ \\
\hline $\begin{array}{l}3360 \\
3234 \\
3185 \\
3241 \\
2975\end{array}$ & $\begin{array}{r}20 \\
15 \\
25 \\
28 \\
9\end{array}$ & $\begin{array}{l}3322 \\
3202 \\
3150 \\
3213 \\
2949\end{array}$ & $\begin{array}{l}0 \\
0 \\
0 \\
0 \\
0\end{array}$ & $\begin{array}{l}3275 \\
3159 \\
3089 \\
3148 \\
2904\end{array}$ & $\begin{array}{l}34 \\
19 \\
30 \\
26 \\
80\end{array}$ & $\begin{array}{l}3007 \\
2872 \\
2799 \\
2858 \\
2555\end{array}$ & $\begin{array}{l}25 \\
45 \\
22 \\
55 \\
29\end{array}$ & $\begin{array}{l}2921 \\
2787 \\
2710 \\
2750 \\
2452\end{array}$ & $\begin{array}{l}70 \\
72 \\
33 \\
36 \\
55\end{array}$ & $\begin{array}{l}2565 \\
2475 \\
2409 \\
2472 \\
2162\end{array}$ & $\begin{array}{l}36 \\
21 \\
20 \\
35 \\
16\end{array}$ & $\begin{array}{l}2529 \\
2436 \\
2374 \\
2407 \\
2124\end{array}$ & $\begin{array}{l}65 \\
50 \\
40 \\
40 \\
40\end{array}$ & $\begin{array}{l}2139 \\
2058 \\
1997 \\
2058 \\
1760\end{array}$ & $\begin{array}{r}22 \\
30 \\
20 \\
9 \\
15\end{array}$ & $\begin{array}{l}2117 \\
2028 \\
1977 \\
2049 \\
1724\end{array}$ & $\begin{array}{r}20 \\
10 \\
10 \\
0 \\
0\end{array}$ & $\begin{array}{l}2013 \\
1935 \\
1890 \\
1985 \\
1663\end{array}$ & $\begin{array}{r}65 \\
75 \\
117 \\
50 \\
75\end{array}$ & $\begin{array}{l}1889 \\
1835 \\
1773 \\
1893 \\
1580\end{array}$ & $\begin{array}{r}15 \\
0 \\
0 \\
0 \\
0\end{array}$ & $\begin{array}{l}1920 \\
1832 \\
1787 \\
3393 \\
1840\end{array}$ & $\begin{array}{l}88 \\
86 \\
89\end{array}$ & $\begin{array}{l}M \\
M \\
M \\
M \\
M\end{array}$ \\
\hline $\begin{array}{l}2740 \\
2280\end{array}$ & $\begin{array}{r}9 \\
10\end{array}$ & $\begin{array}{l}2721 \\
2237\end{array}$ & $\begin{array}{l}0 \\
0\end{array}$ & $\begin{array}{l}2657 \\
2186\end{array}$ & $\begin{array}{l}60 \\
80\end{array}$ & $\begin{array}{l}2350 \\
1862\end{array}$ & $\begin{array}{l}37 \\
25\end{array}$ & 1725 & 30 & $\begin{array}{l}1437 \\
1162 \\
1162 \\
1202\end{array}$ & $\begin{array}{l}16 \\
15 \\
20 \\
10\end{array}$ & $\begin{array}{l}1407 \\
1132 \\
1132 \\
1177\end{array}$ & $\begin{array}{l}55 \\
40 \\
20 \\
30\end{array}$ & $\begin{array}{r}1128 \\
874 \\
874 \\
896\end{array}$ & $\begin{array}{l}50 \\
30 \\
35 \\
25\end{array}$ & $\begin{array}{r}1075 \\
830 \\
830 \\
854\end{array}$ & $\begin{array}{l}10 \\
20\end{array}$ & $\begin{array}{l}993 \\
739 \\
739 \\
767\end{array}$ & $\begin{array}{l}60 \\
75 \\
84 \\
60\end{array}$ & $\begin{array}{l}882 \\
655 \\
655 \\
697\end{array}$ & 0 & $\begin{array}{l}1355 \\
2495\end{array}$ & 93 & $\begin{array}{l}K \\
M\end{array}$ \\
\hline $\begin{array}{r}1916 \\
1031 \\
1040 \\
915\end{array}$ & $\begin{array}{l}15 \\
15 \\
15 \\
20\end{array}$ & $\begin{array}{r}1882 \\
975 \\
989 \\
851\end{array}$ & $\begin{array}{r}10 \\
0 \\
0 \\
0\end{array}$ & $\begin{array}{r}1765 \\
835 \\
853 \\
669\end{array}$ & $\begin{array}{l}40 \\
45 \\
50 \\
40\end{array}$ & $\begin{array}{r}1394 \\
520 \\
533 \\
268\end{array}$ & $\begin{array}{l}45 \\
60 \\
45 \\
45\end{array}$ & $\begin{array}{r}1270 \\
383 \\
397 \\
177\end{array}$ & $\begin{array}{l}35 \\
25 \\
20 \\
45\end{array}$ & $\begin{array}{r}989 \\
80 \\
104 \\
-74\end{array}$ & $\begin{array}{l}25 \\
20 \\
20 \\
30\end{array}$ & $\begin{array}{r}952 \\
52 \\
74 \\
-116\end{array}$ & $\begin{array}{l}20 \\
15 \\
20 \\
40\end{array}$ & $\begin{array}{r}697 \\
-193 \\
-179 \\
-349\end{array}$ & $\begin{array}{l}35 \\
76 \\
31 \\
81\end{array}$ & $\begin{array}{r}636 \\
-269 \\
-210 \\
-430\end{array}$ & $\begin{array}{r}5 \\
0 \\
5 \\
15\end{array}$ & $\begin{array}{r}569 \\
-338 \\
-316 \\
-479\end{array}$ & $\begin{array}{l}60 \\
72 \\
55 \\
50\end{array}$ & $\begin{array}{r}438 \\
-470 \\
-451 \\
-615\end{array}$ & $\begin{array}{r}8 \\
12 \\
20 \\
23\end{array}$ & $\begin{array}{l}3105 \\
3253 \\
4243 \\
4351\end{array}$ & $\begin{array}{r}89 \\
84 \\
96 \\
105\end{array}$ & $\begin{array}{l}M \\
M \\
M\end{array}$ \\
\hline $\begin{array}{l}849 \\
902\end{array}$ & $\begin{array}{l}13 \\
16\end{array}$ & $\begin{array}{l}738 \\
828\end{array}$ & $\begin{array}{l}10 \\
10\end{array}$ & $\begin{array}{l}566 \\
619\end{array}$ & $\begin{array}{l}45 \\
42\end{array}$ & $\begin{array}{l}209 \\
233\end{array}$ & $\begin{array}{l}38 \\
48\end{array}$ & $\begin{array}{r}95 \\
131\end{array}$ & $\begin{array}{l}50 \\
56\end{array}$ & $\begin{array}{l}-154 \\
-122\end{array}$ & $\begin{array}{l}20 \\
20\end{array}$ & $\begin{array}{l}-196 \\
-152\end{array}$ & $\begin{array}{l}18 \\
15\end{array}$ & $\begin{array}{l}-395 \\
-359\end{array}$ & $\begin{array}{l}64 \\
56\end{array}$ & $\begin{array}{l}-459 \\
-415\end{array}$ & $\begin{array}{l}10 \\
10\end{array}$ & $\begin{array}{l}-534 \\
-464\end{array}$ & $\begin{array}{l}40 \\
45\end{array}$ & $\begin{array}{l}-687 \\
-636\end{array}$ & $\begin{array}{l}0 \\
0\end{array}$ & $\begin{array}{l}4565 \\
3833\end{array}$ & $\begin{array}{r}109 \\
98\end{array}$ & $M$ \\
\hline 437 & 15 & 422 & 10 & 128 & 20 & -126 & 40 & -366 & 82 & -574 & 62 & -636 & 0 & -788 & 10 & -798 & 15 & & & & & $\begin{array}{l}1834 \\
3750\end{array}$ & 105 & $\begin{array}{l}k \\
J\end{array}$ \\
\hline $\begin{array}{l}423 \\
424\end{array}$ & $\begin{array}{l}25 \\
20\end{array}$ & & & & & & & -550 & 124 & -805 & & -910 & & & & & & & & & & & & $\begin{array}{l}k \\
k\end{array}$ \\
\hline 60 & 25 & -94 & 0 & -357 & 0 & $\begin{array}{l}-737 \\
-851\end{array}$ & $\begin{array}{l}38 \\
10\end{array}$ & $\begin{array}{l}-775 \\
-874\end{array}$ & $\begin{array}{l}124 \\
111\end{array}$ & $\begin{array}{l}-1005 \\
-11117\end{array}$ & $\begin{array}{r}105 \\
94\end{array}$ & $\begin{array}{l}-1111 \\
-1211\end{array}$ & 50 & -1219 & 55 & & & -1280 & 40 & -1680 & 70 & 6350 & 138 & $M$ \\
\hline & & & & & & -1419 & 10 & -1435 & 42 & -1477 & & & & & & & & & & & & & & \\
\hline $\begin{array}{l}-677 \\
-943\end{array}$ & $\begin{array}{l}30 \\
35\end{array}$ & $\begin{array}{r}-812 \\
-1067\end{array}$ & 0 & $\begin{array}{l}-1054 \\
-1293\end{array}$ & 0 & $\begin{array}{l}-1363 \\
-1471 \\
-1558\end{array}$ & $\begin{array}{l}20 \\
20 \\
20\end{array}$ & $\begin{array}{l}-1390 \\
-1516 \\
-1589\end{array}$ & $\begin{array}{r}62 \\
116 \\
90\end{array}$ & $\begin{array}{l}-1600 \\
-1734 \\
-1833\end{array}$ & $\begin{array}{l}40 \\
94 \\
70\end{array}$ & $\begin{array}{l}-1654 \\
-1828 \\
-1926\end{array}$ & 85 & $\begin{array}{l}-1846 \\
-2111\end{array}$ & 55 & & & $\begin{array}{l}-1900 \\
-2173\end{array}$ & 35 & $\begin{array}{l}-2425 \\
-2684\end{array}$ & $\begin{array}{l}110 \\
140\end{array}$ & $\begin{array}{l}8819 \\
8064\end{array}$ & $\begin{array}{l}172 \\
160\end{array}$ & $\Gamma$ \\
\hline $\begin{array}{r}-856 \\
-634 \\
-1963 \\
-2276\end{array}$ & $\begin{array}{l}30 \\
25 \\
60 \\
30\end{array}$ & $\begin{array}{r}-991 \\
-775 \\
-2116 \\
-2441\end{array}$ & $\begin{array}{l}0 \\
0 \\
0 \\
0\end{array}$ & $\begin{array}{l}-1223 \\
-1014 \\
\\
-2335 \\
-2652\end{array}$ & $\begin{array}{l}0 \\
0\end{array}$ & $\begin{array}{l}-1448 \\
-1234 \\
-2155 \\
-2527 \\
-2831\end{array}$ & $\begin{array}{l}30 \\
40 \\
65 \\
35 \\
55\end{array}$ & $\begin{array}{l}-1501 \\
-1292 \\
-2225 \\
-2570 \\
-2899\end{array}$ & $\begin{array}{r}100 \\
86 \\
95 \\
84 \\
94\end{array}$ & $\begin{array}{l}-1725 \\
-1540 \\
-2481 \\
-2804 \\
-3151\end{array}$ & $\begin{array}{l}35 \\
50 \\
70 \\
50 \\
60\end{array}$ & $\begin{array}{l}-1779 \\
-1600 \\
-2577 \\
-2871 \\
-3235\end{array}$ & $\begin{array}{r}100 \\
75 \\
80 \\
150 \\
105\end{array}$ & $\begin{array}{l}-2074 \\
-1005 \\
-2850 \\
-3218 \\
-3523\end{array}$ & $\begin{array}{l}40 \\
60 \\
65 \\
80 \\
55\end{array}$ & & & $\begin{array}{l}-2129 \\
-1870 \\
-2930 \\
-3322 \\
-3597\end{array}$ & $\begin{array}{l}45 \\
55 \\
55 \\
65 \\
60\end{array}$ & $\begin{array}{l}-2626 \\
-2430 \\
-3408 \\
-3792 \\
-4076\end{array}$ & $\begin{array}{l}145 \\
155 \\
140 \\
140 \\
125\end{array}$ & $\begin{array}{r}8149 \\
6589 \\
11320 \\
12037 \\
12519\end{array}$ & $\begin{array}{r}158 \\
70 \\
\\
244\end{array}$ & $\begin{array}{l}T \\
T \\
T \\
T \\
T\end{array}$ \\
\hline $\begin{array}{r}-2292 \\
-2457 \\
2939 \\
3332 \\
3396\end{array}$ & $\begin{array}{l}30 \\
30 \\
18 \\
27 \\
25\end{array}$ & $\begin{array}{r}-2462 \\
-2650 \\
2906 \\
3299 \\
3364\end{array}$ & $\begin{array}{r}0 \\
0 \\
0 \\
0 \\
25\end{array}$ & $\begin{array}{r}-2669 \\
-2873 \\
2853 \\
3241 \\
3316\end{array}$ & $\begin{array}{r}0 \\
0 \\
35 \\
33 \\
45\end{array}$ & $\begin{array}{r}-2849 \\
-3025 \\
2622 \\
2965 \\
3026\end{array}$ & $\begin{array}{l}50 \\
20 \\
30 \\
28 \\
50\end{array}$ & $\begin{array}{r}-2922 \\
-3065 \\
2461 \\
2892 \\
2876\end{array}$ & $\begin{array}{l}83 \\
78 \\
60 \\
45 \\
40\end{array}$ & $\begin{array}{r}-3153 \\
-3349 \\
2179 \\
2557 \\
2610\end{array}$ & $\begin{array}{l}55 \\
50 \\
30 \\
30 \\
25\end{array}$ & $\begin{array}{r}-3252 \\
-3439 \\
2142 \\
2506 \\
2556\end{array}$ & $\begin{array}{r}110 \\
70 \\
55 \\
30 \\
75\end{array}$ & $\begin{array}{r}-3507 \\
-3678 \\
1694 \\
2091 \\
2159\end{array}$ & $\begin{array}{l}55 \\
45 \\
18 \\
17 \\
15\end{array}$ & $\begin{array}{r}-3584 \\
-3735 \\
1676 \\
2074 \\
2133\end{array}$ & $\begin{array}{r}0 \\
0 \\
15 \\
20 \\
15\end{array}$ & $\begin{array}{r}-3599 \\
-3750 \\
1941 \\
2026\end{array}$ & $\begin{array}{l}60 \\
50 \\
100 \\
105\end{array}$ & $\begin{array}{r}-4122 \\
-4313 \\
1822 \\
1913\end{array}$ & $\begin{array}{r}100 \\
105 \\
0 \\
5\end{array}$ & $\begin{array}{r}12531 \\
12959 \\
2693 \\
2283 \\
1932\end{array}$ & $\begin{array}{r}224 \\
245 \\
75 \\
75\end{array}$ & $\begin{array}{l}\mathrm{T} \\
\mathrm{T} \\
\mathrm{J} \\
\mathrm{M} \\
\mathrm{M}\end{array}$ \\
\hline $\begin{array}{l}3255 \\
3380\end{array}$ & $\begin{array}{l}20 \\
14\end{array}$ & $\begin{array}{l}3235 \\
3350\end{array}$ & $\begin{array}{l}5 \\
0\end{array}$ & $\begin{array}{l}3385 \\
3361 \\
3319 \\
3182 \\
3278\end{array}$ & $\begin{array}{l}20 \\
25 \\
25 \\
40 \\
20\end{array}$ & $\begin{array}{l}3101 \\
3081 \\
3024 \\
2875 \\
3010\end{array}$ & $\begin{array}{l}30 \\
30 \\
35 \\
25 \\
30\end{array}$ & $\begin{array}{l}2998 \\
2955 \\
2909 \\
2774 \\
2896\end{array}$ & $\begin{array}{l}45 \\
40 \\
45 \\
25 \\
30\end{array}$ & $\begin{array}{l}2696 \\
2655 \\
2634 \\
2492 \\
2592\end{array}$ & $\begin{array}{l}25 \\
25 \\
30 \\
15 \\
15\end{array}$ & $\begin{array}{l}2647 \\
2610 \\
2596 \\
2462 \\
2550\end{array}$ & $\begin{array}{r}150 \\
75 \\
55 \\
105 \\
52\end{array}$ & $\begin{array}{l}2246 \\
2223 \\
2224 \\
2033 \\
2157\end{array}$ & $\begin{array}{l}15 \\
20 \\
18 \\
20 \\
16\end{array}$ & $\begin{array}{l}2225 \\
2203 \\
2206 \\
2141\end{array}$ & $\begin{array}{l}20 \\
10 \\
15 \\
15\end{array}$ & $\begin{array}{l}2096 \\
2109 \\
2041\end{array}$ & $\begin{array}{l}55 \\
55 \\
80\end{array}$ & $\begin{array}{l}2032 \\
2027 \\
1925\end{array}$ & $\begin{array}{r}5 \\
10 \\
0\end{array}$ & $\begin{array}{l}1672 \\
1718 \\
4152 \\
1506 \\
1816\end{array}$ & $\begin{array}{l}70 \\
84\end{array}$ & $\begin{array}{l}\mathrm{J} \\
\mathrm{M} \\
\mathrm{M} \\
\mathrm{J}\end{array}$ \\
\hline $\begin{array}{l}2341 \\
2157 \\
1994 \\
1792 \\
1389\end{array}$ & $\begin{array}{l}15 \\
15 \\
12 \\
15 \\
15\end{array}$ & $\begin{array}{l}2301 \\
2116 \\
1931 \\
1724 \\
1308\end{array}$ & $\begin{array}{r}30 \\
20 \\
0 \\
10 \\
15\end{array}$ & $\begin{array}{l}2241 \\
2036 \\
1849 \\
1627\end{array}$ & $\begin{array}{l}65 \\
20 \\
50 \\
25\end{array}$ & $\begin{array}{l}1894 \\
1681 \\
1524 \\
1299\end{array}$ & $\begin{array}{r}35 \\
30 \\
53 \\
115\end{array}$ & $\begin{array}{l}1758 \\
1546 \\
1335 \\
1107\end{array}$ & $\begin{array}{l}35 \\
30 \\
30 \\
10\end{array}$ & $\begin{array}{r}1471 \\
1247 \\
1044 \\
835\end{array}$ & $\begin{array}{l}20 \\
20 \\
20 \\
20\end{array}$ & $\begin{array}{r}1445 \\
1219 \\
994 \\
804\end{array}$ & $\begin{array}{l}60 \\
40 \\
10 \\
40\end{array}$ & $\begin{array}{r}1163 \\
883 \\
762 \\
524\end{array}$ & $\begin{array}{r}43 \\
50 \\
62 \\
5\end{array}$ & $\begin{array}{r}1120 \\
831 \\
682 \\
494\end{array}$ & $\begin{array}{r}10 \\
5 \\
15 \\
10\end{array}$ & $\begin{array}{l}562 \\
426\end{array}$ & $\begin{array}{l}55 \\
75\end{array}$ & $\begin{array}{l}507 \\
305\end{array}$ & $\begin{array}{l}20 \\
10\end{array}$ & $\begin{array}{l}1987 \\
2034 \\
2921 \\
2878 \\
1502\end{array}$ & $\begin{array}{l}75 \\
88 \\
\\
93 \\
75\end{array}$ & $\begin{array}{l}\mathrm{J} \\
\mathrm{J} \\
\mathrm{M} \\
\mathrm{M} \\
\mathrm{K}\end{array}$ \\
\hline
\end{tabular}

\footnotetext{
$200675 \mathrm{~K}$ 1906 81 K $1763 \quad x$ 2748 (3) $\begin{array}{lllllllllllllllll}726 & 15 & 632 & 5 & & & & & & & & & & & & \end{array}$

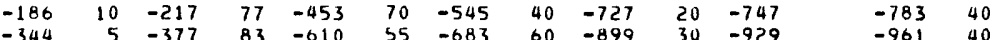


TABLE 2. - SElected geOLOGic data -- CUNTINUEd

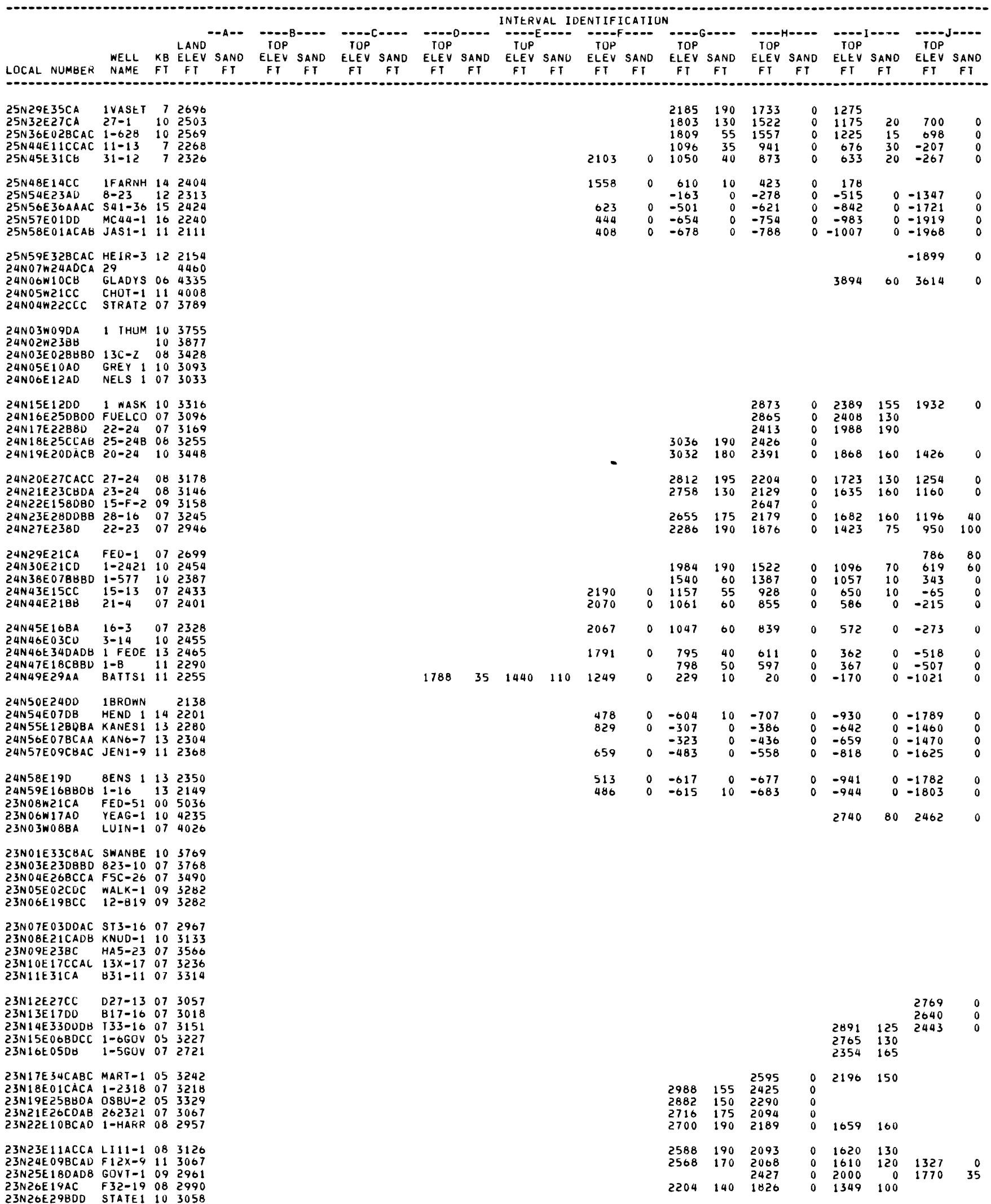




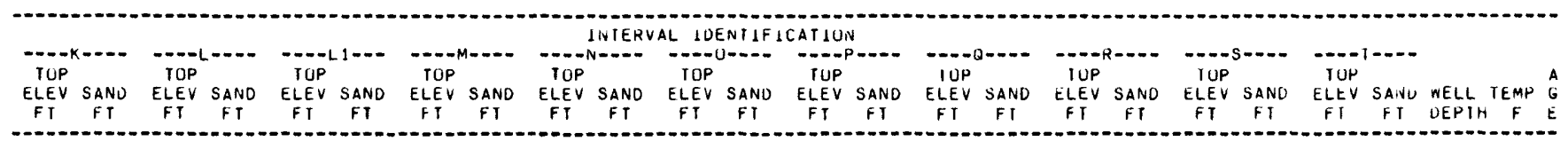

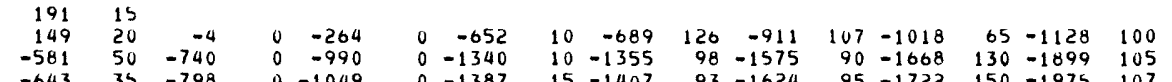

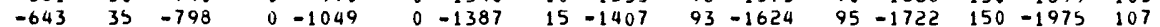

$\begin{array}{lll}-1782 & 30 & -191\end{array}$

$\begin{array}{ll}-2144 & 25-2296 \\ -2319 & 40-2484\end{array}$

$0-2133$

$0-2330$

$75-2411$

$\begin{array}{ll}0 & -2531 \\ 0 & -2703\end{array}$

$\begin{array}{lll}0 & -2710 \\ 0 & -2881\end{array}$

$\begin{array}{ll}55 & -2769 \\ 60 & -2949\end{array}$

$89-2634$

$\begin{array}{llll}0 & -2703 & 0 & -2881 \\ 0 & -2746 & 0 & -2918\end{array}$

$\begin{array}{ll}60 & -2949 \\ 50 & -2978\end{array}$

$65-3031$
86

$86-3186$
$80-3218$

$45-2695$

$60-3111 \quad 70-3361$

$45-3254 \quad 105-3549$

$0-2714$

$\begin{array}{rr}0 & -2890 \\ 30 & 1518\end{array}$

0 2531

$\begin{array}{ll}0 & 2761 \\ 0 & 3242\end{array}$

$30 \quad 2334$

$\begin{array}{rr}30 & -2922 \\ 65 & 1335\end{array}$

$\begin{array}{ll}65 & 1335 \\ 55 & 2163\end{array}$

$\begin{array}{ll}55 & 2163 \\ 38 & 2377\end{array}$

$\begin{array}{rr}83 & -3197 \\ 80 & 1099\end{array}$

$80 \quad 1099$

$\begin{array}{ll}50 & 1873 \\ 55 & 2090\end{array}$

$\begin{array}{rrrr}55 & -3283 & 105 & -3524 \\ 37 & 1002 & 105 & 587\end{array}$

$\begin{array}{rrrr}37 & 1002 & 105 & 587 \\ 36 & 1837 & 175 & 1304\end{array}$

$\begin{array}{llll}36 & 1837 & 175 & 1364 \\ 35 & 2039 & 130 & 1635\end{array}$

$\begin{array}{lll}2837 & 30 & 2802 \\ 3314 & 27 & 3282\end{array}$

$0 \quad 3382$

$45 \quad 2969$

$\begin{array}{lll}3465 & 25 & 3430\end{array}$

$\begin{array}{lll}3492 & 17 & 3463 \\ 2977 & 25 & 2944\end{array}$

$\begin{array}{llll}0 & 3382 & 40 & 3095\end{array}$

442947

$\begin{array}{llll}0 & 2888 & 100 & 2585\end{array}$

$\begin{array}{llll}0 & 2717 & 110 & 2377\end{array}$

303003

$40 \quad 2436$

$\begin{array}{ll}30 & 2267 \\ 92 & 2041\end{array}$

$90 \quad 2067$

$90 \quad 2717$

$\begin{array}{ll}40 & 2166 \\ 30 & 1975\end{array}$

$\begin{array}{lll}2626 & 14 & 2608\end{array}$

$0 \quad 1072$

$124320 \quad 1178$

$881 \quad 10 \quad 822$

$0 \quad 655$

55605

$30 \quad 2587$

$45 \quad 2652$

$\begin{array}{ll}35 & 2122 \\ 13 & 1949\end{array}$

$14 \quad 1749$

$40 \quad 2320$

55
65
657779

$\begin{array}{ll}65 & 1779 \\ 60 & 1607\end{array}$

$23 \quad 259$

$20 \quad 19$

88

$-1425 \quad 30=1555$

0408

25

$5 \quad 151$

$43-82$

$20-120$

$30-362$

$50 \quad-415$

$0 \quad-51$

$80-1954$

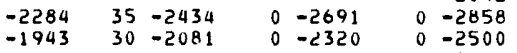

$\begin{array}{lll}-1961 & 30 & -2103 \\ -2138 & 30 & -2291\end{array}$

$\begin{array}{lll}-2288 & 15-2440\end{array}$

$\begin{array}{rrr}50 & -3432 \\ 75 & 3286\end{array}$

$15 \quad 1761$ $\begin{array}{lll}670 & 20 & 615 \\ 460 & 25 & \end{array}$

$309 \quad 10$

$\begin{array}{rrr}142 & 20 & 10 \\ -62 & 10 & -144 \\ -598 & 25 & -78 \\ -674 & 30 & -846\end{array}$

$\begin{array}{rll}-710 & 30 \quad-860\end{array}$

$-916 \quad 35-1058$

$-906 \quad 25-1039$

0

$\begin{array}{ll}10 & -201 \\ 0 & -513\end{array}$

$0-513$
$0-1043$

$\begin{array}{ll}0 & -453 \\ 0 & -906\end{array}$

$0-1412$

$0-1116$

$0-1470$

$\begin{array}{ll}20 & -523 \\ 10 & -934\end{array}$

$0=1122$

$94-1654$

$\begin{array}{rrrr}40 & -926 & 65 & -1159 \\ 81 & -1253 & 25 & -1433\end{array}$

$92-1746 \quad 115-1962$

$0-1463$

$10-1478 \quad 89-1716$

$120-1832 \quad 70=2034$

$0-1308$

$0-1633$

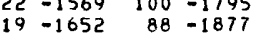

$89-1805$

100
$96-1973$

$25-1953$

$30-1629$

$84-1850$

$60-2096$

$\begin{array}{ll}60 & -2096 \\ 40 & -2093\end{array}$

$0-2053$

$31-2084$

$66-2288$

$85-2373$

$80-2554$

$-2042$

$30-2091$

$69-2309$

$50-2589$

$\begin{array}{ll}0 & -2346 \\ 0 & -2533\end{array}$

$\begin{array}{ll}0 & -2531 \\ 0 & -2706\end{array}$

$60-2616$

$\begin{aligned} 56 & -3172 \\ 62 & -2817 \\ 60 & -2843\end{aligned}$

$60-2843$

$82-2391$

$61-3253 \quad 100=3495$

$60-2902 \quad 110-3137$

$\begin{array}{rlrl}45 & -2925 & 85 & -3153 \\ 88 & -3105 & 100 & -3342\end{array}$

$0-2681$

$0-2857$

$110-2967$

$60-3199$

$73-3280$

$95-3517$

$57-3186$

$98-3301$

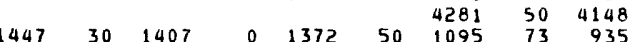

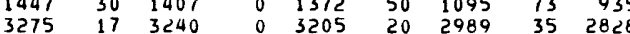

$\begin{array}{rr}50 & 3787 \\ 45 & 696\end{array}$

$21 \quad 675$

$\begin{array}{rr}95 & 172 \\ 80 & 2088\end{array}$

3333

$3117 \quad 63 \quad 2965$

$95 \quad 2919$

$60 \quad 2706$

$35 \quad 2517$

$23 \quad 2884$

$12 \quad 2683$

$40 \quad 2526$

$95 \quad 2345$

$\begin{array}{lllllll}3073 & 18 & 3051 & 0 & 2959 & 73 & 2639\end{array}$

$45 \quad 2447$

$35 \quad 2705$

$\begin{array}{ll}25 & 2229 \\ 25 & 2436\end{array}$

$15 \quad 2206$

$\begin{array}{ll}65 & 2312 \\ 50 & 1811\end{array}$

$\begin{array}{lll}2784 & 25 & 2742\end{array}$

$0 \quad 2649$

$5 \quad 2304$

$40 \quad 2211$

$\begin{array}{ll}66 & 2174\end{array}$

$\begin{array}{ll}25 & 2084 \\ \text { So } & 2053\end{array}$

$\begin{array}{ll}28 & 1867\end{array}$

$\begin{array}{ll}25 & 1803 \\ 20 & 1773\end{array}$

$15 \quad 1820$

352019

$\begin{array}{ll}70 & 1527\end{array}$

$\begin{array}{ll}35 & 1457 \\ 45 & 1447\end{array}$

$\begin{array}{lll}2494 & 21 & 2471 \\ 2401 & 20 & 2381\end{array}$

- 2389

$\begin{array}{ll}80 & 2001 \\ 70 & 1908\end{array}$

$30 \quad 1784$

$20 \quad 1511$

$15 \quad 1481$

$40 \quad 1138$

$\begin{array}{lll}2051 & 12 & 2032 \\ 1944 & 33 & 1900\end{array}$

$0 \quad 1947$

$\begin{array}{llll}70 & 1536 & 77 & 1394\end{array}$

301140

$24 \quad 1082$

$\begin{array}{llll}20 & 947 & 25 & 720 \\ 20 & 773 & 20 & 555\end{array}$

12

$25-1184$ $\begin{array}{ll}30 & -1463 \\ 30 & -1992\end{array}$ $30-1992$
$25-2060$

$57-2010$

$59-2155$

$54=2147$

$18-2572$

$28-3523$

$50-318$

$\begin{array}{ll}40 & -3193 \\ 24 & -3366\end{array}$

$26-3543$

$45-3477$

$\begin{array}{rr}18 & 154 \\ 22 & 2066\end{array}$

$\begin{array}{ll}52 & 2474 \\ 58 & 2285\end{array}$

58 2285

$\begin{array}{ll}60 & 2251 \\ 30 & 1769\end{array}$

$\begin{array}{ll}30 & 1769 \\ 24 & 1995\end{array}$

$\begin{array}{ll}51 & 1476 \\ 26 & 1431\end{array}$

$\begin{array}{ll}26 & 1431 \\ 47 & 1395\end{array}$

$26 \quad 1218$

27111

$\begin{array}{llllll}78 & 753 & 30 & 661 & 70 & 591\end{array}$

$\begin{array}{llllll}80 & 640 & 28 & 525 & 60 & 461 \\ 88 & 467 & 16 & 366 & 40 & 313\end{array}$

$10-3297$

$45-3505$

$55-3701$

$\begin{array}{ll}25 & 2367 \\ 20 & 2180\end{array}$

$20 \quad 2180$

102154

$10 \quad 1881$

$\begin{array}{llll}25 & 1384 & 36 & 1319\end{array}$

$15 \quad 1309 \quad 45 \quad 1239$

$5 \quad 1195$

$\begin{array}{lllllllll}1944 & 33 & 1900 & 0 & 1797 & 49 & 1375 & 56 & 1245 \\ 1741 & 20 & 1700 & 0 & 1631 & 60 & 1202 & 55 & 1073\end{array}$ $30 \quad 2327$

2111
$12 \quad 3263$

0003116

$258874 \mathrm{~K}$

$6896144 \mathrm{M}$

5769
5825
$583 \mathrm{M}$

11664234

65 - 3972 110 12653253 $40-4223 \quad 9012782232$ i

$\begin{array}{rrrrr}35 & -4128 & 85 & 12726 & \\ 85 & 319 & 0 & 4428 & 93\end{array}$ $\begin{array}{llllll}75 & 1107 & 0 & 3494 & 94 & M\end{array}$ $\begin{array}{rrrrrr}75 & 1326 & 0 & 2844 & 89 & M \\ 94 & 1850 & 0 & 2023 & 103 & M\end{array}$

$78 \quad 1989$

$0411791 \mathrm{M}$

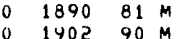
$0176088 \mathrm{M}$ $0374394 \mathrm{M}$

० $453996 \mathrm{M}$ 1943 1843 1674

2540 $\begin{array}{lll}2617 \\ 1553 & 72 & k\end{array}$ $394699 \mathrm{~J}$ $262383 \mathrm{~K}$ 2663

$55-1441 \quad 90 \quad 4137110 \mathrm{M}$

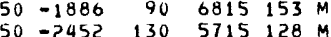
$35-2525$ 75 $5918135 \mathrm{M}$ $50-2538 \quad 65 \quad 5418139 \mathrm{M}$ $\begin{array}{lllll}55 & -2687 \quad 35 \quad 8948 \quad 172 \mathrm{M}\end{array}$ $\begin{array}{lllll}55-3167 & 180 & 6893 & 157 & M\end{array}$

$\begin{array}{lllll}55 & -4073 & 180 & 12736 & 252\end{array}$ $45-3746 \quad 21012091222$ $\begin{array}{lllll}30 & -4168 & 155 & 9423 & 182\end{array}$ $\begin{array}{llllll}60 & -4088 & 210 & 12757 & 225 & \mathrm{~T}\end{array}$ $\begin{array}{rrllll}85 & 3024 & 0 & 3000 & 87 & M \\ 83 & -82 & 0 & 4423 & 98 & M\end{array}$

$\checkmark 2171 \quad M$

$353095 \mathrm{M}$ 1747

$1478 \quad M$
$1700 \quad 79 M$ $\begin{array}{llll}0 & 1700 & 79 & M \\ 0 & 1501 & 80 & M\end{array}$

1698 $0200975 \mathrm{M}$ $240291 \mathrm{M}$ $\begin{array}{llll}0 & 2274 & 98 & M \\ 0 & 2389 & 104 & M\end{array}$ $2549 \quad 88 \mathrm{M}$ 2976
1595 $159076 \mathrm{~K}$ 1630 1968
1697 1793

$\begin{array}{lll}1992 & \mathrm{~K} \\ 3755 & 109 & \mathrm{~J}\end{array}$

$\begin{array}{lll}3755 & 109 & \mathrm{~J} \\ 3246 & 102 \mathrm{~J}\end{array}$

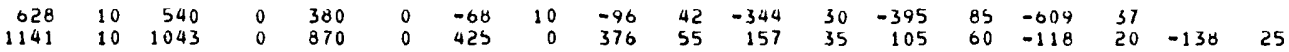

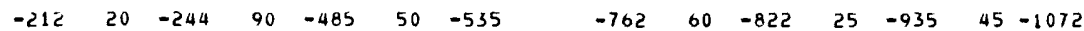

$2085 \quad 73 \mathrm{~K}$ 
table 2. -- SELECTED geologic data -- cuntinued

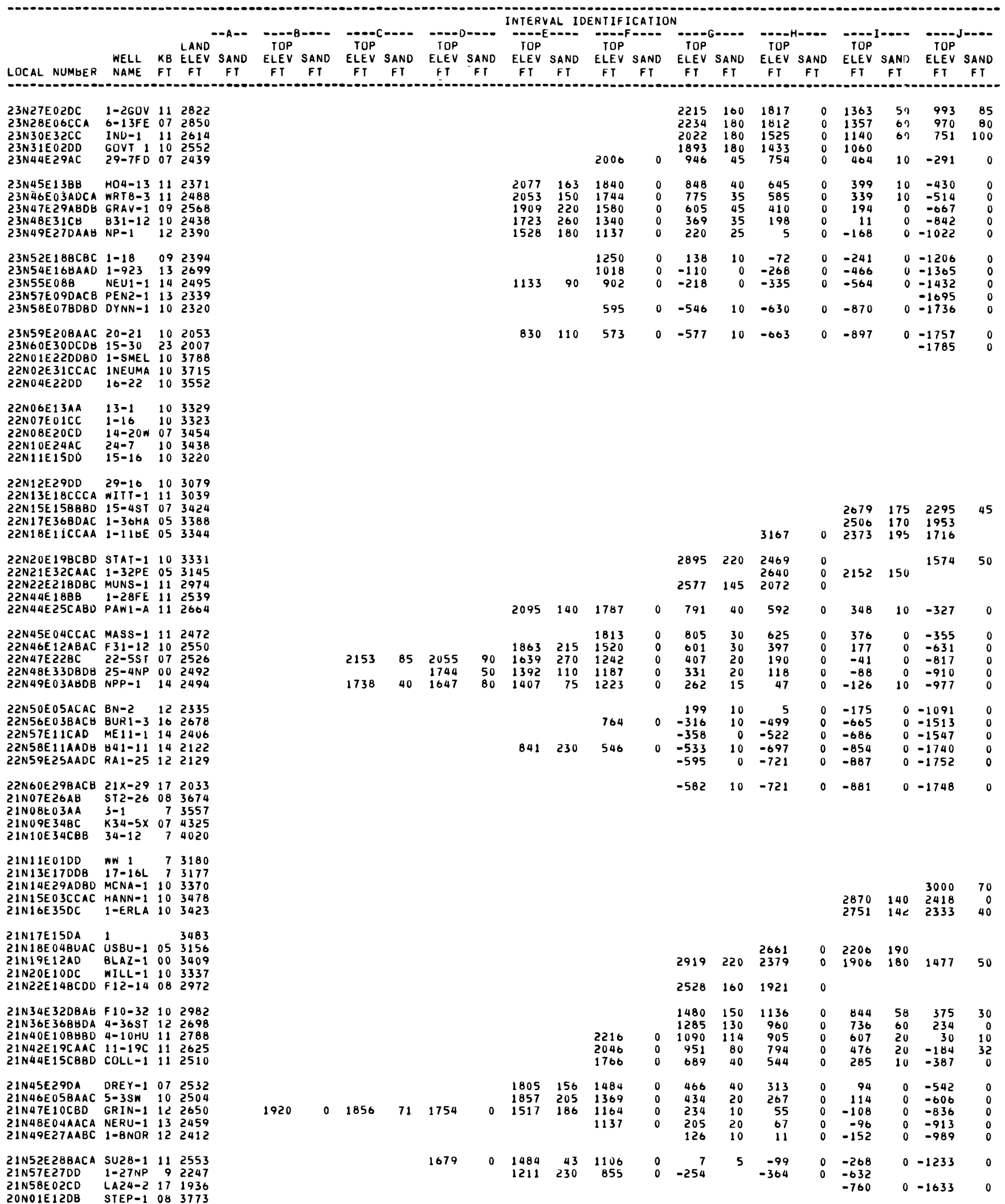


INTERVAL IDENIIFICATION

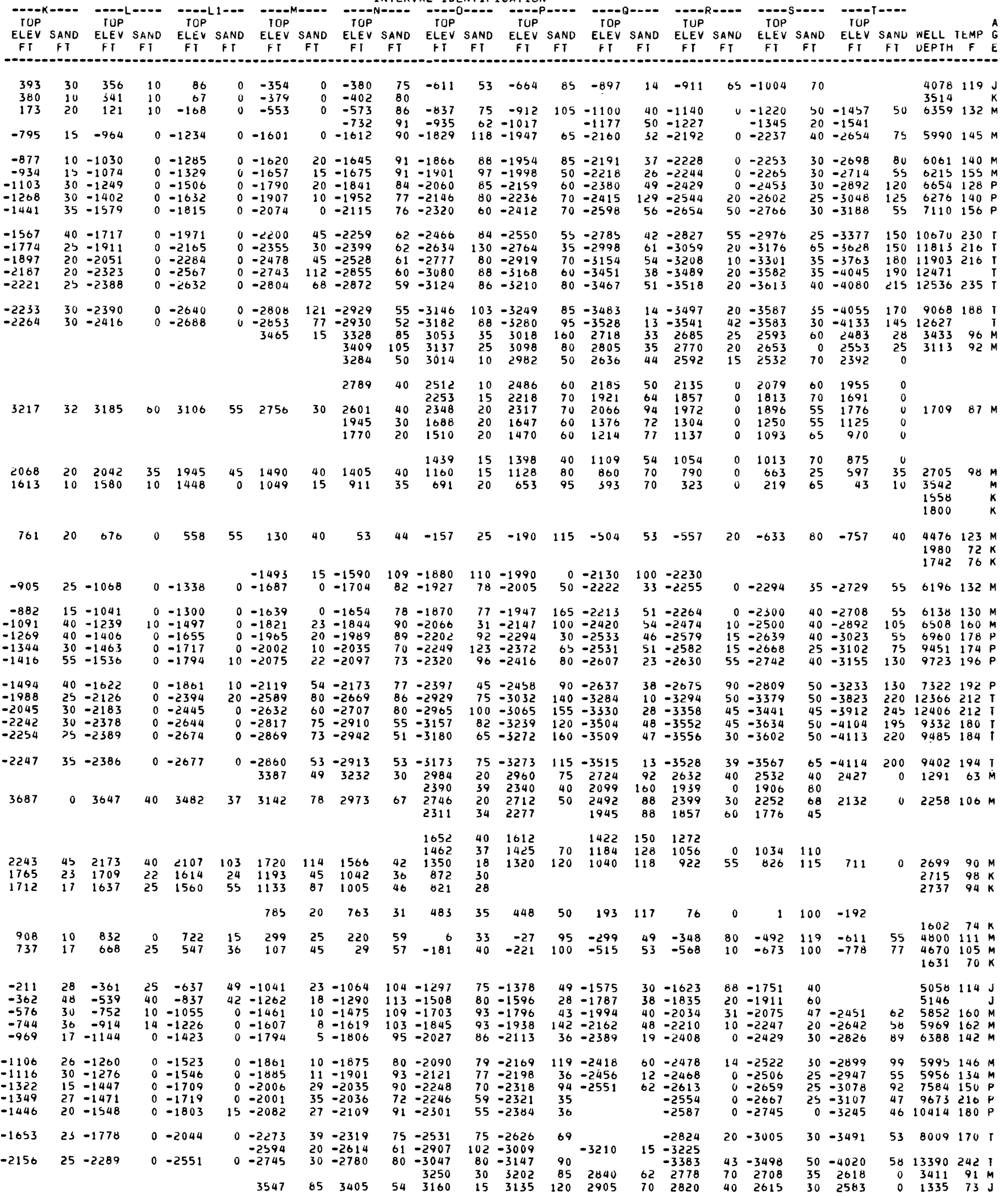


20N10E27DDC 27-16 74516 20N11E34DC L15-34 07390 20N12E 22DCAC 15X-22 O7 365 2ON14E2SCC 23-138 07349 2ON1SE29CDBD COPE-1 073590

20N16E11DOAC 1-LENI 103469 20NI IQE 2AACAC SMIH-1 O5 3456 2ON2OE 2ON22E 29CBAD FD29-1 05 3045 2ON23E14BBD 1-14IN 0B 3050

20N25E04CDA 1-4 2973 2ON25E17 11122924 20N26E14ADCB 1-14CR OB 2800 20N34E 16CDAC 14-103 08 3105 $20 N 40 E 27$ CAAC 11-27B 112752

2ON43E27CBAD HINN-1 122320 20N45EOSDD NP 1 2688 2ON45EO6BBDB SH11-6 102513 20N46E198CAC BNRL- 12 2734

20N4BE09CC 1-9-20 112504 $\begin{array}{lllll}1-9-20 & 11 & 2504 & 190 & 213\end{array}$ 2ON49E13B8 $1-1320112434 \quad 170 \quad 207$ 2ONSOEOAA NPJ9-1 11 2514 200209 2ONSIEO3CCAC NPR3-1 $112539 \quad 300$ 2117

2ON53E078B ACRA-1 112705 2ON54E $36 A D A C$ STAT-1 142778 2ON56EZÜC 1-BLAN 13224 2ON57E3IAC CLAR-1 192383 2ON59E O2ADDB ALU1-2 162255

2ON6OE29CDBO NPRR-1 122267 19N09E33AAAC 33-1 074741 ION1OE21CCAC 21-13 074442 19N11E27CCDE 27-13 073735 19N12E34AABD I VAN 12377 19N13E26A8AC 1 AUGU 123616 19N14E17AOC IDAUPL 19N15E19DÓ 1 11 3640 19N22EOICBAD 1 KOMA O5 309 19N23E25DD IMENDR 183092 $19 N 26 E 2600 B A 1-26$ OB 2980 $\begin{array}{llll}19 N 26 E 2600 B A & 1-26 & 08 & 2980 \\ 19 N 32 E 36 A D B & 8-36 & 12 & 2796\end{array}$ 19N32E36ADB $8-36 \quad 122796$ $\begin{array}{llll}\text { 19N34E32BDBD } 1 \text { SAYL } 11 & 3099 \\ 19 N 36 E 16 A B D B & 216 & 12 & 2870\end{array}$

19N37E10DB 1 RIVE 11282 19N39E21CD 1421 112675 19N41E17DAAC 9-17 112588 ION42E2 $4 A A C A$ B-1 122572 $19 N 43 E 2700 \quad 44-27 \quad 102700$

19N44E07ABCA 31-7 102492 $19 N 47 E 1300$ N11319 112524 $19 N 4 B E 2300 \quad 1-22082569$ I9N49E15CC N1151911 2527 IONSOEOACCAC NPI-23 O8 2677

19N51E25CC 1-251911 2874 19N52E28DEDB HAGN-1 222716 19N52E28DODB HAGN-1 22 2716 19N53E0SC GOSS-1 14 2744 $\begin{array}{llll}\text { 19NS4E30A } & \text { 80JE-1 } & 12 & 2668 \\ \text { ION56E2IAACA } & 1-3 T A T & 10 & 2480\end{array}$

19N59E17CCC 122186 IBNOBE 13CCCA 13-13 07453 IONOAEISBEAC $4-15$ OQ 435 IBNIOE178B8D $17-4 \quad 10417$ IBNIIE298B 29-4 073921

18N12E15AADB 15-1 073838 IBNISE 12CAAC 1SENEF 143592 IBN19E28CODB 14-28 093978 18N20E29DDD 16-29 093933 I8N2IEO5ABOU SAITE 93806

25) $\begin{array}{llllllllll}2051 & 80 & 1737 & 33 & 1675 & 55 & 1412 & 170 & 1035\end{array}$ $\begin{array}{llll}350 & 1967 & 115 & 1664\end{array}$ $\begin{array}{llllllllll}245 & 2023 & 125 & 1736 & 35 & 1688 & 15 & 1479 & 210 & 1000\end{array}$

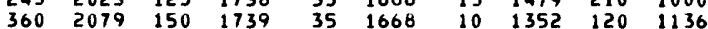
$\begin{array}{llllllllll}570 & 2062 & 100 & 1833 & 155 & 1678 & 40 & 1502 & 125 & 109\end{array}$

\section{0 \\ 451661}

$2438 \quad 140 \quad 2548$
2038

1797

1668

$47 \quad 1771$

$190 \quad 1670$

$215 \quad 1747$

$170 \quad 1694$

$\begin{array}{llll}30 & 1463 & 125 & 1123\end{array}$

$55 \quad 1425 \quad 205 \quad 1115$

$\begin{array}{llll}40 & 1325 & 75 & 11085\end{array}$

1097
1097

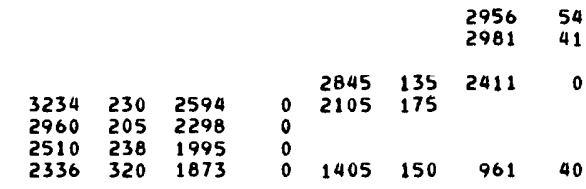

$\begin{array}{lllllll}2173 & 160 & 1688 & 0 & 1275 & 145 & 1043\end{array}$

$\begin{array}{lll}2080 & 172 \quad 1650\end{array}$

$\begin{array}{rrrrr}0 & 1183 & 130 & & \\ 0 & 805 & 0 & 343 & 0 \\ 0 & 573 & 0 & -36 & 0\end{array}$

$\begin{array}{llll}0 & 312 & 0 & -384\end{array}$

$\begin{array}{llll}0 & 228 & 0 & -452\end{array}$

$\begin{array}{llll}0 & 228 & 0 & -452 \\ 0 & -98 & 0 & -688\end{array}$

$\begin{array}{llll}0 & -135 & 0 & -831\end{array}$

$\begin{array}{llll}0 & -134 & 0 & -879\end{array}$

$\begin{array}{llll}0 & -153 & 0 & -991\end{array}$

$0-189 \quad 0-1046$

$\begin{array}{ll}0 & -1120 \\ 0 & -1242\end{array}$

$0-1203 \quad 15$

$\begin{array}{ll}0 & -1299 \\ 0 & -1397\end{array}$

$-16780$

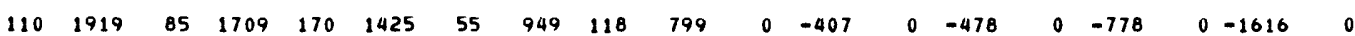

18N23E02008D 44-2 103200

$18 N 24 E 170 B$ 1PESTA 53161

18N24E17DB 1 1PESTA 271611

$\begin{array}{llll}18 N 27 E 27 A A B A & 1-27 & 10 & 3100 \\ 1 B N 3 O E 22 B A & 3-22 & 08 & 2855\end{array}$

$\begin{array}{llll}1 B N 30 E 22 B A & 3-22 & 08 & 2855 \\ 1 B N 32 E 15 C A B D & 11-15 & 08 & 3062\end{array}$

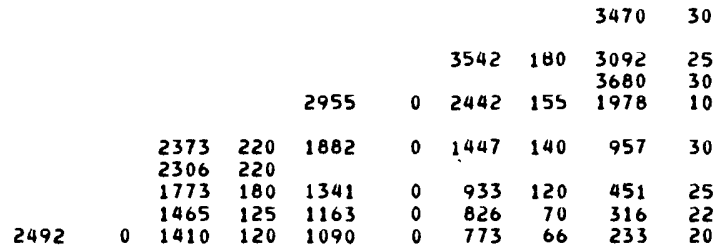


INTERVAL IDENTIFICAIION

TOP ELEV SAND ELEV SAND ELEV SAND ELEV SAND ELEV SAND ELEV SAND ELEV SAND ELEV SAND ELEV SAND ELEV SAND ELEV SAND WELL TEMP G $\begin{array}{ll} & \end{array}$

\begin{tabular}{|c|c|c|c|c|c|c|c|c|c|c|c|c|c|c|c|c|c|c|c|c|c|c|c|}
\hline $\begin{array}{l}3312 \\
2827 \\
2300 \\
2245\end{array}$ & $\begin{array}{l}36 \\
27 \\
28 \\
18\end{array}$ & $\begin{array}{l}3248 \\
2775 \\
2246 \\
2186\end{array}$ & $\begin{array}{l}60 \\
48 \\
42 \\
39\end{array}$ & $\begin{array}{l}3156 \\
2706 \\
2168 \\
2108\end{array}$ & $\begin{array}{l}90 \\
50 \\
40 \\
56\end{array}$ & $\begin{array}{l}2785 \\
2358 \\
1768 \\
1693\end{array}$ & $\begin{array}{l}55 \\
68 \\
60 \\
59\end{array}$ & $\begin{array}{l}2665 \\
2218 \\
1642 \\
1557\end{array}$ & $\begin{array}{l}37 \\
41 \\
42 \\
47\end{array}$ & $\begin{array}{l}2485 \\
2409 \\
1984 \\
1412 \\
1348\end{array}$ & $\begin{array}{l}42 \\
31 \\
31 \\
36 \\
37\end{array}$ & $\begin{array}{l}2443 \\
2378 \\
1953 \\
1376 \\
1311\end{array}$ & $\begin{array}{r}165 \\
145 \\
80 \\
100\end{array}$ & $\begin{array}{r}2101 \\
2003 \\
1591 \\
1065 \\
979\end{array}$ & $\begin{array}{r}30 \\
33 \\
90 \\
105\end{array}$ & $\begin{array}{r}2057 \\
1973 \\
1558 \\
941 \\
857\end{array}$ & $\begin{array}{l}62 \\
30 \\
20 \\
20\end{array}$ & $\begin{array}{r}1871 \\
1809 \\
1448 \\
845 \\
767\end{array}$ & $\begin{array}{l}69 \\
85 \\
93 \\
99\end{array}$ & $\begin{array}{r}1740 \\
752 \\
668\end{array}$ & $\begin{array}{r}40 \\
0 \\
0\end{array}$ & $\begin{array}{l}2328 \\
2394 \\
2852 \\
3055\end{array}$ & $\begin{array}{l}85 \\
84 \\
81 \\
81\end{array}$ \\
\hline 1803 & 15 & 1707 & 22 & 1625 & 40 & 1109 & 10 & 1079 & 33 & 872 & 30 & -632 & 40 & -855 & 71 & -926 & 36 & -1057 & 40 & 1214 & 70 & $\begin{array}{l}2701 \\
1827 \\
1912 \\
1933 \\
4527\end{array}$ & $\begin{array}{r}78 k \\
\\
65 k \\
74 k \\
74 k \\
125\end{array}$ \\
\hline
\end{tabular}

$\begin{array}{lllllllllll}-457 & 10 & -474 & 63 & -696 & 77 & -773 & 80 & -1079 & 20 & -1099\end{array}$

$\begin{array}{rrrrr}-240 & 0 & -397 & 0 & -675 \\ -632 & 10 & -812 & 35 & -1120\end{array}$

$0-1107$

$-1099$

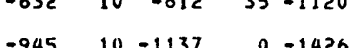

$25-1510$

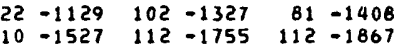

$\begin{array}{ll}60 & -1592 \\ 10 & -2077\end{array}$

$46-1638$
$43-2120$

15

$\begin{array}{llll}-945 & 10-1137 & 0 & -1426\end{array}$

$5-1789$

$\begin{array}{llll}18 & -1807 & 96 & -2021\end{array}$

$93-2120$

$20 \quad-2368$

$18-2386$

$-2180$

$25-2540$

$6061164 \mathrm{M}$

$\begin{array}{lllrl}-1036 & 19 & -1210 & 0 & -1485 \\ -1231 & 19 & -1358 & 0 & -1607 \\ -1335 & 30 & -1447 & 10 & -1695\end{array}$

$7-1847$

$\begin{array}{llllll}0 & -1782 & 83 & -2051 & 92 & -2143\end{array}$

$42-1915$
$0-1982$

$\begin{array}{llll}-102 & -207\end{array}$

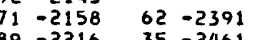

$\begin{array}{lll}-1335 \quad 30 & -1447\end{array}$

$10-1695$

$11-1926$

$8-2127$

-2216
90

$63-2454$

$\begin{array}{ll}0 & -2489\end{array}$

$25-2830$

$535980145 \mathrm{M}$

$\begin{array}{rll}-1443 & 25 & -1465 \\ -1450 & -1543\end{array}$

$0-1720$

$10-2015$

$25-2045$

$90-2254$

71

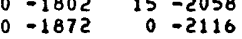

$0-1958$

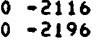

65
$64-2123$
34

$\begin{array}{ll}64 & -2322 \\ 69 & -2387\end{array}$

$62-2578 \quad 66$

$66-25$

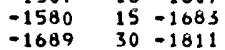

$0-2095$

$0-2323$

$48-2245$
$48-2371$

$61-2457$

77

$\begin{array}{lrl}-1639 & 0 & -1766 \\ -1752 & 30 & -1891\end{array}$

$\begin{array}{ll}0 & -2043 \\ 0 & -2195\end{array}$

$\begin{array}{lll}0 & -2257 \\ 15 & -23916\end{array}$

$\begin{array}{llll}54 & -2311 & 66 & -2538 \\ 48 & -2439 & 59 & -2698\end{array}$

$68-2596$

75
56

-1865 15 -1997

$\begin{array}{ll}0 & -2306 \\ 0 & -254\end{array}$

$15-2391$

$\begin{array}{ll}66 & -2538 \\ 59 & -2698 \\ 64 & -2815\end{array}$

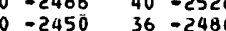

$\begin{array}{ll}0 & -2636 \\ 0 & -2306\end{array}$

$\begin{array}{lll}0 & -2810 \\ 0 & -5450\end{array}$

$\begin{array}{ll}47 & -2486 \\ 17 & -2837\end{array}$

$85-2778$
$78-3118$

$55-2614$

$\begin{aligned} & 90 \\ & 90\end{aligned}-2818$

$62-2860$

$37-2802$

$20-3088$

$32-2834$

20
$30-3108$

$98-3166$

$35-320$

$\begin{array}{lll}55 & -2996 \\ 27 & -3183 & \end{array}$

$27=3183$

$0-3307$
$15-3277$

$\begin{array}{lll}-3277 & 40-3698 \\ 1 & -3596\end{array}$

$35-3494$

$\begin{array}{ll}30 & -3674 \\ 25 & -3788\end{array}$

$85-3216 \quad 38=3457 \quad 24=348$

$\begin{array}{lll}-2142 \quad 22 & -2278 \\ -4988 & 43 & 4445\end{array}$

$0-2584$

$\begin{array}{llll}0 & -2761 \quad 27 & -2788\end{array}$

$80-3056$

$40 \quad 3889$

$\begin{array}{rrrrrr}82 & 4363 & 255 & 4052 & 130 & 3905 \\ 10 & 3808 & 220 & 3439 & 60 & 3301\end{array}$

$\begin{array}{lll}3941 & 40 & 3889 \\ 3508 & 40 & 3449\end{array}$

$\begin{array}{lll}3508 & 40 & 3449 \\ 3196 & 20 & 3142\end{array}$

$\begin{array}{llllll}55 & 3382 & 65 & 3012 & 45 & 2881 \\ 20 & 3075 & 25 & 2693 & 30 & 2607\end{array}$

$44 \quad 3059$

$2798 \quad 15 \quad 2751$

$0 \quad 2691$

$20 \quad 2292$

2292
2067
2016

$\begin{array}{ll}35 & 2647 \\ 40 & 2329\end{array}$

99

$\begin{array}{llll}23 & 3033 & 125 & 2496\end{array}$

$\begin{array}{llll}26 & 3033 & 125 & 2496 \\ 28 & 2619 & 255 & 2187\end{array}$

$90 \quad 2400$

00.2343

$0-4067$

$\begin{array}{llllll}40 & 2147 & 0 & 2343 & 90 & 2234\end{array}$

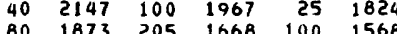

$\begin{array}{llll}15 & 2299 & 135 & 1953\end{array}$

$\begin{array}{ll}7 & 1933 \\ 0 & 1709\end{array}$

$\begin{array}{llll}10 & 1896 \quad 145 \quad 1542\end{array}$

$\begin{array}{llll}25 & 1677 & 90 & 1340 \\ 30 & 1662 & 60 & 1289\end{array}$

$\begin{array}{rrrrrrr}54 & 1488 & 150 & 1310 & 96 & 1214 \\ 53 & 1287 & & 1137 & 100 & 1009\end{array}$

$\begin{array}{lllll}53 & 1287 & & 1137 & 100 \\ 38 & 1251 & 30 & 1046 & 103\end{array}$

$30 \quad 1961$

$42 \quad 1699$

$\begin{array}{lllll}-321 & 10 & -347 & 60 & -548\end{array}$

$0-1103$

$0-1107$

$\begin{array}{lrlll}-250 & 0 & -292 & 0 & -655 \\ -250 & 30 & -297 & 0 & -660 \\ -433 & 25 & -488 & 0 & -873\end{array}$

$\begin{array}{ll}0 & -660 \\ 0 & -873\end{array}$

$\begin{array}{ll}35 & -555 \\ 3 & -730\end{array}$

$0 \quad-953$

$\begin{array}{ll}0 & -1142 \\ 0 & -1299\end{array}$

$\begin{array}{lll}0 & -1299 \\ 0 & -1499 & \end{array}$

$\begin{array}{rr}0 & -1499 \\ 0 & -1546\end{array}$

$\begin{array}{lll}-1013 & 25 & -878 \\ -10050 & 20 & -1081\end{array}$

$\begin{array}{lll}-1013 & 25 & -1081 \\ -1050 & 20 & -1117\end{array}$

$-1055 \quad 20-1130$

$\begin{array}{lll}-1441 & 45-1548\end{array}$

$\begin{array}{lll}-1579 & 35 & -1675 \\ -1500 & 20 & -1596\end{array}$

$0-1535$

$0-1808$

$\begin{array}{ll}0 & -1952 \\ 0 & -1871\end{array}$

$\begin{array}{ll}0 & -1871 \\ 0 & -1825\end{array}$

$0-2005$

$0-1982$

$0-1949$
0.1974

$\begin{array}{ll}0 & -1974 \\ 0 & -2274\end{array}$

$-1599 \quad 40-1679$

$-1533 \quad 35-1599$

$\begin{array}{ll}-1547 & 25-1633 \\ -1816 & 40-1917\end{array}$

$0-1356$

$0=-1545$

$0=1686$

$0=1869$
$0=1914$

$0-1903$

$\begin{array}{lll}0 & -1903 \\ 0 & -2107 & -2237\end{array}$

$\begin{array}{ll}0 & -2237 \\ 0 & -2136\end{array}$

$\begin{array}{ll}0 & -2136 \\ 0 & -2095\end{array}$

$0-2247$

$0=-2216$

$0-2149$

$0=2460$

$-2639$

$-2639$

3800

3583

$3287 \quad 25 \quad 3185$

$\begin{array}{rrr}2723 & 17 & 2699 \\ 2512 & 0 & 2424\end{array}$

$\begin{array}{rrr}3172 & 0 & 3097 \\ 1431 & 10 & 1311\end{array}$

$\begin{array}{lll}426 & 0 & 306\end{array}$

$\begin{array}{lll}-46 & 0 & -167\end{array}$

$\begin{array}{lll}-181 & 0 & -299 \\ -248 & 0 & -371\end{array}$
$0 \quad 3107$

0
0 2626

$\begin{array}{ll}0 & 2315 \\ 0 & 2995\end{array}$

- 1202

$\begin{array}{lr}0 & 150 \\ 0 & -368\end{array}$

$\begin{array}{ll}0 & -549 \\ 0 & -649\end{array}$
$20 \quad 2763$

$60 \quad 2188$

$45 \quad 2536$

$35-311$

$25 \quad-783$

$\begin{array}{lr}36 & -963 \\ 27 & -1051\end{array}$ $\begin{array}{llll}0 & -1120 & 112 & -1333 \\ 0 & -1124 & 120 & -1330\end{array}$

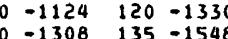

$\begin{array}{llll}0 & -1376 \quad 119-1594\end{array}$

$0-1558 \quad 132-1796$

$0=-1698 \quad 113-1929$

$0-1883 \quad 102-2101$

$\begin{array}{rrrr}0 & -1883 & 102 & -2101 \\ 0 & -1927 & 96 & -2139\end{array}$

$0-1915$

$\begin{array}{rlll}0 & -1915 & 87 & -2131 \\ 10 & -2128 & 94 & -2338\end{array}$

$\begin{array}{llll}30 & -2270 & 84 & -2336 \\ & 86 & -2477\end{array}$

$\begin{array}{llll}15 & -2181 & 92 & -2394\end{array}$

-

$\begin{array}{ll}40 & -2292 \\ 43 & -2259\end{array}$

$\begin{array}{llll}15 & -2194 & 57 & -2422\end{array}$

$\begin{array}{llll}49 & -2259 & 60 & -2483 \\ 35 & -2495 & 59 & -2779\end{array}$

35 - 2495

$57-2957$

$27-2666$

$121 \quad 3999$$$
\text { (3) }
$$

$\begin{array}{rr}42 & 4145 \\ 3736\end{array}$

$\begin{array}{ll}42 & 3736 \\ 31 & 3411\end{array}$

$55 \quad 3528$

$33 \quad 3221$

$\begin{array}{llll}89 & 2674 & 40 & 2398 \\ 40 & 2096 & 39 & 1843\end{array}$

$\begin{array}{llll}25 & 1737 & 44 & 1573 \\ 45 & 2451 & 45 & 2301\end{array}$

$20 \quad 655$

$\begin{array}{ll}42 & -353 \\ 20 & -394\end{array}$

$\begin{array}{ll}20 & -394 \\ 66 & -849\end{array}$

$\begin{array}{lll}66 & -1029 \\ 20 & -1112\end{array}$

$48 \quad 499$

$\begin{array}{ll}55 & -534 \\ 58 & -575\end{array}$

$76-1026$

$90-1218$
$97-1308$
$70-618$
-40

$69-1402$

$80-1410$

$79-1673$

$74-1870$

$83-2012$

$95-2196$
$104-2243$

$40 \quad-872$

$80-1641$

$55-1630$
$55-1833$

$80-1872$

$\begin{array}{ll}20 & -2054 \\ 50 & -2189\end{array}$

$\begin{array}{ll}50 & -2189 \\ 35 & -2394\end{array}$

$35-2394$
$40-2438$

$82-2213 \quad 25-2432$

67
80

$\begin{array}{llll}95 & -2502 & 40 & -2644\end{array}$

$71-2455 \quad 30=2589$

$\begin{array}{lllll}75 & -2579 & 120 & -2783\end{array}$

$\begin{array}{rrrr}54 & -2560 & 60 & -2728 \\ 56 & -2478 & 60 & -2687\end{array}$

$\begin{array}{llll}56 & -2478 & 60 & -2687 \\ 45 & -2528 & 10 & -2753\end{array}$

$77-2896$

$30=3130$

$98-3069$

$\begin{array}{llll}20 & 4118 & 245 & 3680\end{array}$

$\begin{array}{llll}20 & 3709 & 235 & 3320\end{array}$

$\begin{array}{llll}20 & 3382 & 240 & 3011\end{array}$

$\begin{array}{llll}30 & 2365 & 110 & 1966\end{array}$

$\begin{array}{llll}30 & 1796 & 70 & 1395\end{array}$

$\begin{array}{llll}55 & 1514 & 135 & 1199 \\ 55 & 2242 & 125 & 1925\end{array}$ $\begin{array}{ll}79 & -613 \\ 78 & -653\end{array}$

$\begin{array}{llll}78 & -653 & 50 & -841\end{array}$

$\begin{array}{llll}76 & -1102 & 118 & -1363\end{array}$

$\begin{array}{llll}77 & -1295 & 86 & -1539 \\ 78 & -1386 & 82 & -1609\end{array}$

$\begin{array}{ll}75 & -947\end{array}$

$56-1697$

$\begin{array}{ll}56 & -1680 \\ 54 & -1887\end{array}$

$50-1922$

$56-2110$

$94-2283$

$\begin{array}{ll}85 & -2479 \\ 44 & -2482\end{array}$

$46-2488$

22
44

19
$39-2767$
42

$42-2729$ $33-27$

$-1107$

$-1228$

50

$20-1840$

$20-2034$

$\begin{aligned} & 0-2204 \\ & 5\end{aligned}-2345$

$5-2345$
$-20-2536$

$20-2536$
$45-2587$ 


\begin{tabular}{|c|c|c|c|c|c|c|c|c|c|c|c|c|c|c|c|c|c|c|c|c|c|c|}
\hline LOCAL NUMBER & $\begin{array}{l}\text { WELL } \\
\text { NAME }\end{array}$ & $\begin{array}{l}K B \\
F T\end{array}$ & $\begin{array}{l}\text { LAND } \\
\text { ELEV } \\
\text { FT }\end{array}$ & $\begin{array}{l}\text {-AM- } \\
\text { SANO } \\
\text { FT }\end{array}$ & $\begin{array}{l}\text { TUP } \\
\text { ELEV } \\
\text { FT }\end{array}$ & $\begin{array}{l}\text { SANO } \\
\text { FT }\end{array}$ & $\begin{array}{l}\text { TUP } \\
\text { ELEV } \\
\text { FT }\end{array}$ & $\begin{array}{l}\text { SANO } \\
\text { FT }\end{array}$ & $\begin{array}{c}\text { TOP } \\
\text { ELEV } \\
F I\end{array}$ & SAND & $\begin{array}{l}\text { INTERY } \\
\text { TOP } \\
\text { ELEY } \\
\text { FI }\end{array}$ & $\begin{array}{l}\text { VAL IO } \\
\text { EAND } \\
\text { SAT } \\
\text { FT }\end{array}$ & \begin{tabular}{l} 
ENTIF \\
\hdashline TOP \\
ELEV \\
FT
\end{tabular} & $\begin{array}{l}\text { ICATIC } \\
\text { F...- } \\
\text { SANO } \\
\text { FI }\end{array}$ & \begin{tabular}{l} 
N TOP \\
ELEV \\
\hdashline$I$
\end{tabular} & $\begin{array}{l}\text { GANO } \\
\text { ST }\end{array}$ & $\begin{array}{l}\text { IOP } \\
\text { ELEV } \\
\text { FI }\end{array}$ & $\begin{array}{l}\text { SANO } \\
\text { FT }\end{array}$ & $\begin{array}{l}\text { TUP } \\
\text { ELEV } \\
\text { FT }\end{array}$ & $\begin{array}{l}\text { SAAD } \\
\text { FT }\end{array}$ & $\begin{array}{l}\text { TUP } \\
\text { ELEV }\end{array}$ & $\begin{array}{l}\text { SAND } \\
\text { FT }\end{array}$ \\
\hline $\begin{array}{l}18 N 33 E 16 C B \\
18 N 42 E 24 D U \\
18 N 43 E O 2 D D \\
18 N 4433 B C \\
18 N 49 E 27 C C A C\end{array}$ & $\begin{array}{l}16-12 \\
1 P A D O \\
44-2 \\
B N-H-1 \\
1-19 N P\end{array}$ & $\begin{array}{l}10 \\
11 \\
11 \\
20 \\
08\end{array}$ & $\begin{array}{l}3184 \\
2562 \\
2521 \\
2650 \\
2832\end{array}$ & 425 & 2032 & 80 & 1710 & 5 & 1612 & 5 & $\begin{array}{l}2699 \\
1688 \\
1610 \\
1435\end{array}$ & $\begin{array}{l}110 \\
110 \\
120 \\
170\end{array}$ & $\begin{array}{r}2476 \\
1408 \\
1302 \\
985\end{array}$ & $\begin{array}{l}0 \\
0 \\
0 \\
0\end{array}$ & $\begin{array}{r}1377 \\
557 \\
375 \\
82\end{array}$ & $\begin{array}{r}150 \\
25 \\
25 \\
0\end{array}$ & $\begin{array}{r}1059 \\
323 \\
199 \\
-4\end{array}$ & $\begin{array}{l}0 \\
0 \\
0 \\
0\end{array}$ & $\begin{array}{r}740 \\
95 \\
-51 \\
-291\end{array}$ & $\begin{array}{r}20 \\
0 \\
25 \\
0\end{array}$ & $\begin{array}{r}189 \\
-590 \\
-698 \\
-1123\end{array}$ & $\begin{array}{l}0 \\
0 \\
0 \\
0\end{array}$ \\
\hline $\begin{array}{l}18 N 49 E 36 D B \\
18 N 50 E 21 B B \\
18 N 53 E 22 C C \\
1 B N 56 E 1986 B C \\
18 N 58 E 0900\end{array}$ & $\begin{array}{l}\text { STATEI } \\
\text { NP-1 } \\
14-22 \\
1-19 \\
\text { NP } 1\end{array}$ & $\begin{array}{r}9 \\
11 \\
12 \\
12 \\
12\end{array}$ & $\begin{array}{l}3143 \\
2824 \\
2633 \\
2442 \\
2263\end{array}$ & 560 & 1978 & 80 & 1638 & 80 & 1521 & 20 & 1150 & 135 & 952 & 0 & $\begin{array}{r}55 \\
647 \\
273\end{array}$ & $\begin{array}{r}0 \\
12 \\
0\end{array}$ & $\begin{array}{l}-37 \\
523 \\
168\end{array}$ & $\begin{array}{l}0 \\
0 \\
0\end{array}$ & $\begin{array}{r}-325 \\
127 \\
-166\end{array}$ & $\begin{array}{l}0 \\
0 \\
0\end{array}$ & $\begin{array}{r}-1185 \\
-854 \\
-1189\end{array}$ & $\begin{array}{l}0 \\
0 \\
0\end{array}$ \\
\hline $\begin{array}{l}\text { 18N59E17AA } \\
18 N 60 E 05 A A \\
17 N 03 E 05 D D B D \\
17 N 12 E 17 D D A C \\
17 N 13 E 15 A A\end{array}$ & $\begin{array}{ll}N P & 1 \\
N P & 1 \\
N P & 1 \\
17-16 \\
15-1\end{array}$ & $\begin{array}{r}12 \\
12 \\
7 \\
7 \\
7\end{array}$ & $\begin{array}{l}2282 \\
2328 \\
3853 \\
4032 \\
3962\end{array}$ & & & & & & & & & & & & & & & & & & & \\
\hline $\begin{array}{l}\text { 17N14E32BC } \\
17 N 15 E 09 D D B D \\
17 N 1 B E 14 C B B D \\
17 N 19 E 01 C C \\
17 N 21 E 35 A D\end{array}$ & $\begin{array}{l}1000-1 \\
9-16 \\
12-14 \\
1-13 G 1 \\
F R 35-8\end{array}$ & $\begin{array}{r}12 \\
7 \\
9 \\
09 \\
10\end{array}$ & $\begin{array}{l}3762 \\
3571 \\
40,11 \\
4515 \\
3804\end{array}$ & & & & & & & & & & & & & & & & & & $\begin{array}{l}3610 \\
3960\end{array}$ & $\begin{array}{l}52 \\
35\end{array}$ \\
\hline $\begin{array}{l}17 N 22 E 16 A A O B \\
17 N 23 E 3108 \\
17 N 24 E 17 \mathrm{CC} \\
17 N 25 E 2700 \\
17 N 31 E 080 A C C\end{array}$ & $\begin{array}{l}16-1 S T \\
\text { CRI-31 } \\
1 \text { DELA } \\
\text { FUHS-1 } \\
9-8\end{array}$ & $\begin{array}{l}09 \\
10 \\
11 \\
11 \\
12\end{array}$ & $\begin{array}{l}3716 \\
3334 \\
3152 \\
3013 \\
2939\end{array}$ & & & & & & & & & & 2507 & 0 & 1372 & 140 & 1103 & 0 & 734 & 60 & $\begin{array}{r}3490 \\
215\end{array}$ & 30 \\
\hline $\begin{array}{l}17 N 33 E \text { 17DACC } \\
17 N 36 E 100 C \\
17 N 39 E 28 C A \\
17 N 40 E 260 B \\
17 N 44 E 10 A 0\end{array}$ & $\begin{array}{l}13-4 \\
\text { F } 38 G-1 \\
\text { CHAP-1 } \\
\text { 1SCHMI } \\
1\end{array}$ & $\begin{array}{l}10 \\
00 \\
11 \\
12 \\
12\end{array}$ & $\begin{array}{l}3246 \\
2872 \\
2800 \\
2885 \\
2645\end{array}$ & & & & & & & & $\begin{array}{l}2592 \\
2239 \\
2107\end{array}$ & $\begin{array}{l}140 \\
115 \\
124\end{array}$ & $\begin{array}{l}2376 \\
2188 \\
1995 \\
1729 \\
1027\end{array}$ & $\begin{array}{l}0 \\
0 \\
0 \\
0 \\
0\end{array}$ & $\begin{array}{r}1246 \\
1045 \\
801 \\
677 \\
75\end{array}$ & $\begin{array}{r}150 \\
86 \\
70 \\
20\end{array}$ & $\begin{array}{l}954 \\
770 \\
625 \\
507 \\
-73\end{array}$ & $\begin{array}{l}0 \\
0 \\
0 \\
0\end{array}$ & $\begin{array}{l}603 \\
526 \\
325 \\
260\end{array}$ & $\begin{array}{l}30 \\
40 \\
2^{4}\end{array}$ & $\begin{array}{r}84 \\
-87 \\
-313\end{array}$ & $\begin{array}{r}0 \\
20 \\
0\end{array}$ \\
\hline $\begin{array}{l}\text { 17N4SE } 33 C A \\
17 N 48 E 01 C C \\
17 N 49 E 1700 \\
17 N 5203 A D \\
17 N 53 E 22 A C\end{array}$ & $\begin{array}{l}\text { NPRR } 1 \\
\text { I-18 } \\
\text { IHUBIN } \\
\text { ISCHWA } \\
\text { NEWA-1 }\end{array}$ & $\begin{array}{l}14 \\
08 \\
11 \\
12\end{array}$ & $\begin{array}{l}2833 \\
2886 \\
3086 \\
2623 \\
2495\end{array}$ & 570 & 2005 & 50 & $\begin{array}{l}1917 \\
1834\end{array}$ & $\begin{array}{l}100 \\
125\end{array}$ & $\begin{array}{l}1787 \\
1675\end{array}$ & $\begin{array}{l}15 \\
40\end{array}$ & $\begin{array}{l}1467 \\
1327 \\
\\
2067\end{array}$ & $\begin{array}{l}105 \\
145\end{array}$ & $\begin{array}{l}1207 \\
1041 \\
1795\end{array}$ & $\begin{array}{l}0 \\
0\end{array}$ & $\begin{array}{l}353 \\
192 \\
\\
1224\end{array}$ & $\begin{array}{r}10 \\
0\end{array}$ & $\begin{array}{r}195 \\
71 \\
1205\end{array}$ & $\begin{array}{r}0 \\
10\end{array}$ & $\begin{array}{r}-80 \\
-226 \\
409\end{array}$ & $\begin{array}{r}2 n \\
0\end{array}$ & $\begin{array}{r}-822 \\
-1035 \\
-589\end{array}$ & $\begin{array}{l}0 \\
0\end{array}$ \\
\hline $\begin{array}{l}17 N 54 E 2500 B 0 \\
\text { I } 7 N 55 E 19 C B C A \\
17 N 59 E 278 B \\
16 N 13 E 18 A B \\
\text { ION15E } 30 A B\end{array}$ & $\begin{array}{l}\text { UNDEMI } \\
\text { UNDEM } 1 \\
\text { NP } 1 \\
31-18 \\
1-O E N Z\end{array}$ & $\begin{array}{l}13 \\
12 \\
12 \\
10 \\
12\end{array}$ & $\begin{array}{l}2489 \\
2611 \\
2560 \\
4464 \\
4077\end{array}$ & & & & & & & & & & & & & & & & $\begin{array}{l}137 \\
130\end{array}$ & $\begin{array}{l}0 \\
0\end{array}$ & $\begin{array}{l}-945 \\
-944\end{array}$ & $\begin{array}{l}0 \\
0\end{array}$ \\
\hline $\begin{array}{l}\text { ION1 } 6 E 07 B A A C \\
16 N 21 E 03 A A \\
\text { ION21EOTOA } \\
16 N 22 E 06 A A B D \\
\text { I ON26E } 23 B B A C\end{array}$ & $\begin{array}{l}\text { I-WICH } \\
\text { IFIELD } \\
\text { IRICHA } \\
6-1 F R A \\
1-K O O T\end{array}$ & $\begin{array}{l}12 \\
10 \\
10 \\
10 \\
10\end{array}$ & $\begin{array}{l}3751 \\
3854 \\
4059 \\
3707 \\
3050\end{array}$ & & & & & & & & & & & & & & & & & & & \\
\hline $\begin{array}{l}16 N 27 E 26 B C B \\
16 N 28 E 09 B 0 B D \\
16 N 29 E 22 C B D A \\
16 N 31 E 23 D C D B \\
16 N 34 E 12 A C C A\end{array}$ & $\begin{array}{l}\text { SKIB-1 } \\
22-9 \\
12-22 \\
\text { MAIO-1 } \\
\text { HOOK-1 }\end{array}$ & $\begin{array}{l}03 \\
10 \\
08 \\
12 \\
10\end{array}$ & $\begin{array}{l}2939 \\
3005 \\
2619 \\
2995 \\
3090\end{array}$ & & & & & & & & & & $\begin{array}{l}2445 \\
2386\end{array}$ & $\begin{array}{l}0 \\
0\end{array}$ & $\begin{array}{l}1578 \\
1543 \\
1479 \\
1390\end{array}$ & $\begin{array}{l}120 \\
140 \\
150 \\
135\end{array}$ & $\begin{array}{l}1288 \\
1266 \\
1014 \\
907\end{array}$ & $\begin{array}{l}0 \\
0 \\
0 \\
0\end{array}$ & $\begin{array}{l}875 \\
861 \\
707 \\
645\end{array}$ & $\begin{array}{r}125 \\
129 \\
99 \\
59\end{array}$ & $\begin{array}{l}389 \\
359 \\
200 \\
168\end{array}$ & $\begin{array}{l}0 \\
0 \\
0 \\
0\end{array}$ \\
\hline $\begin{array}{l}16 N 36 E 26 \\
16 N 36 E 28 C A \\
16 N 37 E 16 B 8 \\
16 N 38 E 28 B A \\
16 N 40 E 15 B A B O\end{array}$ & $\begin{array}{l}1 \\
1 \text { SONQU } \\
4-16 \\
1-N C T 1 \\
\text { NPRR } 1\end{array}$ & $\begin{array}{l}12 \\
08 \\
12 \\
12\end{array}$ & $\begin{array}{l}3037 \\
3107 \\
2872 \\
2943 \\
2880\end{array}$ & & & & & & & & 2280 & 150 & 1871 & 0 & $\begin{array}{r}2011 \\
1652 \\
2051 \\
921\end{array}$ & $\begin{array}{r}130 \\
130 \\
85 \\
90\end{array}$ & $\begin{array}{r}1234 \\
1650 \\
537\end{array}$ & $\begin{array}{l}0 \\
0 \\
0\end{array}$ & $\begin{array}{r}1020 \\
1431 \\
278\end{array}$ & $\begin{array}{l}57 \\
27 \\
15\end{array}$ & $\begin{array}{r}490 \\
908 \\
-400\end{array}$ & $\begin{array}{l}0 \\
0 \\
0\end{array}$ \\
\hline $\begin{array}{l}16 N 41 E 17 B B \\
16 N 42 E 34 C C C \\
16 N 49 E 17 D U A C \\
16 N 53 E O 2 A A \\
16 N 54 E 21 C B\end{array}$ & $\begin{array}{l}18 \mathrm{~N} \\
14-34 \\
1-17 N P \\
1 \\
1\end{array}$ & $\begin{array}{l}07 \\
15 \\
06 \\
13 \\
14\end{array}$ & $\begin{array}{l}2849 \\
2725 \\
3373 \\
2613 \\
2376\end{array}$ & 780 & 2211 & & & 105 & 1816 & 15 & $\begin{array}{l}1871 \\
1541\end{array}$ & $\begin{array}{r}115 \\
80\end{array}$ & $\begin{array}{l}1458 \\
1109\end{array}$ & 0 & $\begin{array}{l}681 \\
470 \\
258\end{array}$ & $\begin{array}{l}60 \\
50 \\
20\end{array}$ & $\begin{array}{r}311 \\
124\end{array}$ & $\begin{array}{l}0 \\
0\end{array}$ & $\begin{array}{r}76 \\
-176\end{array}$ & $\begin{array}{r}27 \\
0\end{array}$ & $\begin{array}{r}-420 \\
-1048\end{array}$ & $\begin{array}{l}0 \\
0\end{array}$ \\
\hline $\begin{array}{l}\text { 16N54E } 33 B 0 C A \\
1 \text { ON56EOICCAC } \\
16 N 57 E 34000 \\
15 N 12 E 22 C D A C \\
\text { ISN1 } 4 E 34 D D\end{array}$ & $\begin{array}{l}22-23 \\
1-N P R R \\
44-34 P \\
\text { GIFF-1 } \\
\text { WATS-1 }\end{array}$ & $\begin{array}{l}15 \\
12 \\
12 \\
10 \\
09\end{array}$ & $\begin{array}{l}2477 \\
2407 \\
2588 \\
4534 \\
4137\end{array}$ & & & & & & & & & & & & 1022 & 0 & 962 & 0 & $\begin{array}{r}602 \\
41 \\
185\end{array}$ & $\begin{array}{l}0 \\
0 \\
0\end{array}$ & $\begin{array}{r}-432 \\
-1036 \\
-880\end{array}$ & $\begin{array}{l}0 \\
0 \\
0\end{array}$ \\
\hline $\begin{array}{l}\text { 15N15E 13DO } \\
15 N 16 E 120 C \\
15 N 16 E 29 C A \\
15 N 17 E 16 A C \\
\text { ISN21E21AC }\end{array}$ & $\begin{array}{l}D U-A-1 \\
1=12 \\
1 P O S P I \\
\text { JENNI } \\
I=21\end{array}$ & $\begin{array}{l}08 \\
10 \\
10 \\
10 \\
10\end{array}$ & $\begin{array}{l}3843 \\
3924 \\
3865 \\
3924 \\
4048\end{array}$ & & & & & & & & & & & & & & & & & & & \\
\hline $\begin{array}{l}\text { 15N23E22CCB } \\
15 N 24 E 15 C C \\
15 N 25 E 14 A B \\
15 N 27 E 14 D D \\
15 N 28 E 01 C A B A\end{array}$ & $\begin{array}{l}\text { RYAN } 1 \\
\text { ITEIGA } \\
\text { A-IGIA } \\
\text { IGUENO } \\
1 \text {-UCLI }\end{array}$ & $\begin{array}{r}5 \\
5 \\
5 \\
7 \\
70\end{array}$ & $\begin{array}{l}3442 \\
3273 \\
3303 \\
2852 \\
2878\end{array}$ & & & & & & & & & & & & & & & & & & & \\
\hline
\end{tabular}




\begin{tabular}{|c|c|c|c|c|c|c|c|c|c|c|c|c|c|c|c|c|c|c|c|c|c|c|c|c|}
\hline $\begin{array}{l}\text { TUP } \\
\text { ELEV } \\
F T\end{array}$ & $\begin{array}{l}\text { SAND } \\
\text { FT }\end{array}$ & $\begin{array}{l}\text { TOP } \\
\text { ELEV } \\
F T\end{array}$ & $\begin{array}{l}\text { SAND } \\
\text { FI }\end{array}$ & $\begin{array}{l}\text { TOP } \\
\text { ELEV } \\
\text { FT }\end{array}$ & $\begin{array}{l}\text { SAND } \\
\text { FT }\end{array}$ & $\begin{array}{l}\text { TOP } \\
\text { ELEV } \\
\text { FT }\end{array}$ & $\begin{array}{l}\text { SAND } \\
\text { FT }\end{array}$ & $\begin{array}{l}\text { TOP } \\
\text { ELEV } \\
F T\end{array}$ & $\begin{array}{c}\text { SAND } \\
\text { F T }\end{array}$ & $\begin{array}{l}\text { TOP } \\
\text { ELEV } \\
F T\end{array}$ & $\begin{array}{l}\text { SAND } \\
\text { FT }\end{array}$ & $\begin{array}{l}\text { TUP } \\
\text { ELEV } \\
\text { FT }\end{array}$ & $\begin{array}{l}\text { SAND } \\
\text { FT }\end{array}$ & $\begin{array}{l}\text { TOP } \\
\text { ELEV } \\
\text { FT }\end{array}$ & $\begin{array}{c}\text { SAND } \\
\text { F T }\end{array}$ & $\begin{array}{l}\text { TOP } \\
\text { ELEV } \\
\text { FT }\end{array}$ & $\begin{array}{l}\text { SAND } \\
\text { FT }\end{array}$ & $\begin{array}{l}\text { TOP } \\
\text { ELEV } \\
F T\end{array}$ & $\begin{array}{l}\text { SAND } \\
\text { FT }\end{array}$ & $\begin{array}{l}\text { TUP } \\
\text { ELEV } \\
\text { FI }\end{array}$ & $\begin{array}{c}\text { SANO } \\
\text { FT }\end{array}$ & $\begin{array}{l}\text { WELL } \\
\text { DEPIH }\end{array}$ & $\begin{array}{l}\text { TEMP } \\
-\quad F\end{array}$ & $\begin{array}{l}A \\
G \\
E\end{array}$ \\
\hline $\begin{array}{r}-301 \\
-1061 \\
-1148 \\
-1650\end{array}$ & $\begin{array}{r}0 \\
15 \\
0\end{array}$ & $\begin{array}{r}-413 \\
-1162 \\
-1248 \\
-1740\end{array}$ & $\begin{array}{l}0 \\
0 \\
0\end{array}$ & $\begin{array}{r}-173 \\
-1562 \\
-1571 \\
-2032\end{array}$ & $\begin{array}{r}0 \\
0 \\
20 \\
30\end{array}$ & $\begin{array}{l}-1110 \\
-1813 \\
-1792 \\
-2078 \\
-2286\end{array}$ & $\begin{array}{r}63 \\
68 \\
57 \\
5 \\
60\end{array}$ & $\begin{array}{l}-1173 \\
-1881 \\
-1849 \\
-2095 \\
-2348\end{array}$ & $\begin{array}{r}110 \\
109 \\
83 \\
93 \\
110\end{array}$ & $\begin{array}{l}-1381 \\
-2173 \\
-2115 \\
-2284 \\
-2561\end{array}$ & $\begin{array}{l}84 \\
67 \\
43 \\
55 \\
83\end{array}$ & $\begin{array}{l}-1465 \\
-2240 \\
-2208 \\
-2348\end{array}$ & $\begin{array}{l}25 \\
25 \\
23\end{array}$ & $\begin{array}{l}-1660 \\
-2454 \\
-2407\end{array}$ & $\begin{array}{l}68 \\
72 \\
65\end{array}$ & $\begin{array}{l}-1728 \\
-2526 \\
-2472\end{array}$ & $\begin{array}{l}15 \\
20 \\
10\end{array}$ & $\begin{array}{l}-1903 \\
-2597 \\
-2554\end{array}$ & $\begin{array}{l}35 \\
15 \\
20\end{array}$ & $\begin{array}{l}-2123 \\
-2975 \\
-2904\end{array}$ & $\begin{array}{l}55 \\
50 \\
75\end{array}$ & $\begin{array}{l}7706 \\
8772 \\
7705 \\
5612\end{array}$ & $\begin{array}{l}151 \\
2170 \\
145 \\
140 \\
2126\end{array}$ & $\begin{array}{l}M \\
M\end{array}$ \\
\hline \multirow[t]{3}{*}{$\begin{array}{l}-1711 \\
-1315 \\
-1676\end{array}$} & $\begin{array}{r}0 \\
30 \\
25\end{array}$ & $\begin{array}{l}-1809 \\
-1427 \\
-1800\end{array}$ & $\begin{array}{l}0 \\
0 \\
0\end{array}$ & $\begin{array}{l}-2107 \\
-1735 \\
-2127\end{array}$ & $\begin{array}{l}0 \\
0 \\
0\end{array}$ & $\begin{array}{l}-2286 \\
-2368 \\
-1954 \\
-2320 \\
-2498\end{array}$ & $\begin{array}{l}50 \\
52 \\
41 \\
34 \\
17\end{array}$ & $\begin{array}{l}-2344 \\
-2420 \\
-1995 \\
-2360 \\
-2515\end{array}$ & $\begin{array}{r}120 \\
111 \\
60 \\
60 \\
59\end{array}$ & $\begin{array}{l}-2568 \\
-2642 \\
-2239 \\
-2626 \\
-2815\end{array}$ & $\begin{array}{r}70 \\
85 \\
78 \\
70 \\
110\end{array}$ & $\begin{array}{l}-2653 \\
-2317 \\
-2696 \\
-2925\end{array}$ & $\begin{array}{l}55 \\
33\end{array}$ & $\begin{array}{l}-2706 \\
-2532 \\
-3019\end{array}$ & $\begin{array}{l}40 \\
41 \\
27\end{array}$ & $\begin{array}{l}-2796 \\
-2574 \\
-3046\end{array}$ & $\begin{array}{l}30 \\
30\end{array}$ & $\begin{array}{l}-2728 \\
-3138\end{array}$ & $\begin{array}{l}30 \\
25\end{array}$ & $\begin{array}{l}-3203 \\
-3613\end{array}$ & $\begin{array}{l}35 \\
50\end{array}$ & $\begin{array}{r}5739 \\
10685 \\
11098\end{array}$ & $\begin{array}{l}922 \\
5 \\
212 \\
3 \\
196\end{array}$ & $\begin{array}{l}K \\
T \\
T\end{array}$ \\
\hline & & & & & & $\begin{array}{l}-2616 \\
-2730\end{array}$ & $\begin{array}{l}25 \\
25\end{array}$ & $\begin{array}{l}-2651 \\
-2761\end{array}$ & $\begin{array}{l}63 \\
52\end{array}$ & $\begin{array}{l}-2929 \\
-3023\end{array}$ & $\begin{array}{r}92 \\
109\end{array}$ & $\begin{array}{l}-3021 \\
-3132\end{array}$ & & & & & & & & & & & & \\
\hline & 52 & 3303 & 0 & 3287 & 64 & $\begin{array}{l}3681 \\
2857\end{array}$ & $\begin{array}{l}66 \\
20\end{array}$ & $\begin{array}{l}3577 \\
2754\end{array}$ & $\begin{array}{l}40 \\
40\end{array}$ & $\begin{array}{l}3323 \\
2529\end{array}$ & $\begin{array}{l}30 \\
40\end{array}$ & $\begin{array}{l}3287 \\
2482\end{array}$ & $\begin{array}{l}160 \\
175\end{array}$ & $\begin{array}{l}2891 \\
2094\end{array}$ & $\begin{array}{l}125 \\
125\end{array}$ & $\begin{array}{l}3587 \\
2760 \\
1969\end{array}$ & $\begin{array}{l}95 \\
97 \\
93\end{array}$ & $\begin{array}{l}3405 \\
2540 \\
1764\end{array}$ & $\begin{array}{r}70 \\
176 \\
150\end{array}$ & $\begin{array}{l}3285 \\
2364 \\
1584\end{array}$ & $\begin{array}{r}0 \\
90 \\
75\end{array}$ & $\begin{array}{r}979 \\
2934 \\
3650\end{array}$ & $\begin{array}{l}88 \\
78 \\
95\end{array}$ & $\begin{array}{l}M \\
p \\
P\end{array}$ \\
\hline $\begin{array}{l}3146 \\
30110 \\
3418 \\
3356\end{array}$ & $\begin{array}{l}19 \\
24 \\
33 \\
30\end{array}$ & $\begin{array}{l}3096 \\
2945 \\
3348 \\
3263\end{array}$ & $\begin{array}{l}5 \\
20 \\
20 \\
25\end{array}$ & $\begin{array}{l}3532 \\
3070 \\
2841 \\
3241 \\
3121\end{array}$ & $\begin{array}{l}75 \\
42 \\
50 \\
50 \\
25\end{array}$ & $\begin{array}{l}3119 \\
2643 \\
2353 \\
2777 \\
2634\end{array}$ & $\begin{array}{l}25 \\
40 \\
60 \\
50 \\
16\end{array}$ & $\begin{array}{l}3019 \\
2560 \\
2260 \\
2674 \\
2582\end{array}$ & $\begin{array}{l}41 \\
40 \\
46 \\
46 \\
50\end{array}$ & $\begin{array}{l}2779 \\
2318 \\
2084 \\
2508 \\
2406\end{array}$ & $\begin{array}{l}30 \\
48 \\
59 \\
66 \\
75\end{array}$ & $\begin{array}{l}2741 \\
2270 \\
2025 \\
2442 \\
2331\end{array}$ & $\begin{array}{l}112 \\
120 \\
173 \\
183 \\
160\end{array}$ & $\begin{array}{l}2317 \\
1918 \\
1644 \\
2104 \\
2041\end{array}$ & $\begin{array}{r}62 \\
98 \\
72 \\
126 \\
159\end{array}$ & $\begin{array}{l}2254 \\
1820 \\
1572 \\
1971 \\
1882\end{array}$ & $\begin{array}{r}30 \\
100 \\
36 \\
72 \\
90\end{array}$ & $\begin{array}{l}1985 \\
1593 \\
1333 \\
1732 \\
1657\end{array}$ & $\begin{array}{r}75 \\
62 \\
185 \\
178 \\
83\end{array}$ & $\begin{array}{l}1882 \\
1432 \\
1248 \\
1554 \\
1567\end{array}$ & $\begin{array}{r}100 \\
145 \\
55 \\
0 \\
55\end{array}$ & $\begin{array}{l}3344 \\
3308 \\
3227 \\
3206 \\
2902\end{array}$ & $\begin{array}{l}75 \\
84 \\
94\end{array}$ & $\begin{array}{l}P \\
P \\
M \\
M \\
P\end{array}$ \\
\hline $\begin{array}{l}2915 \\
3115 \\
2316 \\
-296\end{array}$ & $\begin{array}{r}35 \\
0 \\
20 \\
15\end{array}$ & $\begin{array}{l}2803 \\
2998 \\
2202 \\
-399\end{array}$ & $\begin{array}{r}35 \\
0 \\
0 \\
30\end{array}$ & $\begin{array}{l}2642 \\
2824 \\
1978 \\
-668\end{array}$ & $\begin{array}{l}20 \\
25 \\
40 \\
50\end{array}$ & $\begin{array}{r}2174 \\
2324 \\
2839 \\
1381 \\
-1118\end{array}$ & $\begin{array}{l}15 \\
10 \\
15 \\
41 \\
20\end{array}$ & $\begin{array}{r}2125 \\
2275 \\
2790 \\
1340 \\
-1141\end{array}$ & $\begin{array}{l}51 \\
50 \\
61 \\
81 \\
88\end{array}$ & $\begin{array}{r}1928 \\
2093 \\
2610 \\
1121 \\
-1336\end{array}$ & $\begin{array}{r}75 \\
83 \\
77 \\
111 \\
81\end{array}$ & $\begin{array}{r}1853 \\
2010 \\
2533 \\
1010 \\
-1417\end{array}$ & $\begin{array}{r}178 \\
162 \\
132 \\
62 \\
150\end{array}$ & $\begin{array}{r}1475 \\
1684 \\
2196 \\
743 \\
-1687\end{array}$ & $\begin{array}{r}108 \\
54 \\
100 \\
149 \\
69\end{array}$ & $\begin{array}{r}1307 \\
1610 \\
2096 \\
-1756\end{array}$ & $\begin{array}{r}100 \\
45 \\
50 \\
\\
20\end{array}$ & $\begin{array}{l}1074 \\
1294 \\
1909\end{array}$ & $\begin{array}{l}48 \\
50 \\
36\end{array}$ & $\begin{array}{r}986 \\
1187 \\
1796\end{array}$ & $\begin{array}{l}45 \\
05 \\
60\end{array}$ & $\begin{array}{l}3636 \\
2794 \\
4531 \\
3278 \\
4893\end{array}$ & $\begin{array}{r}95 \\
110 \\
122 \\
96 \\
117\end{array}$ & $\begin{array}{l}M \\
M \\
M \\
M \\
J\end{array}$ \\
\hline $\begin{array}{l}-424 \\
-570 \\
-816\end{array}$ & $\begin{array}{l}10 \\
10 \\
10\end{array}$ & $\begin{array}{l}-507 \\
-703 \\
-892\end{array}$ & $\begin{array}{r}0 \\
20 \\
16\end{array}$ & $\begin{array}{r}-848 \\
-1080 \\
-1293\end{array}$ & $\begin{array}{l}75 \\
62 \\
55\end{array}$ & $\begin{array}{l}-1294 \\
-1515 \\
-1688 \\
-1786 \\
-2123\end{array}$ & $\begin{array}{l}19 \\
17 \\
15 \\
10 \\
5\end{array}$ & $\begin{array}{l}-1313 \\
-1532 \\
-1703 \\
-1803 \\
-2143\end{array}$ & $\begin{array}{l}121 \\
120 \\
123 \\
113 \\
111\end{array}$ & $\begin{array}{l}-1526 \\
-1746 \\
-1921 \\
-2011 \\
-2335\end{array}$ & $\begin{array}{r}84 \\
89 \\
81 \\
84 \\
109\end{array}$ & $\begin{array}{l}-1610 \\
-1835 \\
-2002 \\
-2095 \\
-2444\end{array}$ & $\begin{array}{l}65 \\
87 \\
70 \\
20 \\
50\end{array}$ & $\begin{array}{l}-1830 \\
-2038 \\
-2213 \\
-2273 \\
-2624\end{array}$ & $\begin{array}{r}111 \\
114 \\
69 \\
80 \\
89\end{array}$ & $\begin{array}{l}-1941 \\
-2152 \\
-2282 \\
-2353 \\
-2713\end{array}$ & $\begin{array}{r}15 \\
12 \\
0 \\
35\end{array}$ & $\begin{array}{l}-2027 \\
-2239 \\
-2392\end{array}$ & $\begin{array}{l}30 \\
40 \\
30\end{array}$ & $\begin{array}{l}-2310 \\
-2536 \\
-2718\end{array}$ & $\begin{array}{l}40 \\
82 \\
70\end{array}$ & $\begin{array}{l}7989 \\
8262 \\
6135\end{array}$ & $\begin{array}{l}175 \\
158 \\
148\end{array}$ & $\begin{array}{c}M \\
M\end{array}$ \\
\hline $\begin{array}{l}-1294 \\
-1559\end{array}$ & $\begin{array}{l}20 \\
38\end{array}$ & $\begin{array}{l}-1353 \\
-1656\end{array}$ & $\begin{array}{r}26 \\
0\end{array}$ & $\begin{array}{l}-1717 \\
-1955\end{array}$ & $\begin{array}{l}86 \\
50\end{array}$ & $\begin{array}{l}-2040 \\
-2238 \\
-2273 \\
-2143 \\
-1723\end{array}$ & $\begin{array}{l}20 \\
26 \\
30 \\
25 \\
23\end{array}$ & $\begin{array}{l}-2060 \\
-2264 \\
-2312 \\
-2187 \\
-1746\end{array}$ & $\begin{array}{r}102 \\
85 \\
113 \\
59 \\
60\end{array}$ & $\begin{array}{l}-2249 \\
-2484 \\
-2526 \\
-2366 \\
-1989\end{array}$ & $\begin{array}{l}112 \\
118 \\
122 \\
101 \\
107\end{array}$ & $\begin{array}{l}-2361 \\
-2602 \\
-2648 \\
-2467 \\
-2096\end{array}$ & $\begin{array}{l}60 \\
30 \\
20 \\
15\end{array}$ & $\begin{array}{l}-2627 \\
-2181\end{array}$ & $\begin{array}{l}25 \\
45\end{array}$ & $\begin{array}{l}-2652 \\
-2226\end{array}$ & 10 & -2360 & 15 & -2966 & 232 & $\begin{array}{l}9848 \\
5671\end{array}$ & $\begin{array}{l}175 \\
124\end{array}$ & $\begin{array}{l}P \\
k\end{array}$ \\
\hline \multirow[t]{2}{*}{$\begin{array}{l}-1418 \\
-1415\end{array}$} & $\begin{array}{l}40 \\
45\end{array}$ & $\begin{array}{l}-1549 \\
-1547\end{array}$ & $\begin{array}{r}0 \\
30\end{array}$ & $\begin{array}{r}-1888 \\
-1885 \\
\\
3536\end{array}$ & $\begin{array}{l}0 \\
0\end{array}$ & $\begin{array}{r}-2085 \\
-2081 \\
-2418 \\
3872 \\
3123\end{array}$ & $\begin{array}{l}20 \\
30 \\
25 \\
25 \\
31\end{array}$ & $\begin{array}{r}-2118 \\
-2133 \\
-2468 \\
3811 \\
3079\end{array}$ & $\begin{array}{l}62 \\
60 \\
57 \\
28 \\
35\end{array}$ & $\begin{array}{r}-2392 \\
-2383 \\
-2745 \\
3526 \\
2841\end{array}$ & $\begin{array}{l}98 \\
87 \\
97 \\
48 \\
45\end{array}$ & $\begin{array}{r}-2490 \\
-2470 \\
-2842 \\
3478 \\
2791\end{array}$ & $\begin{array}{r}10 \\
8 \\
90 \\
125\end{array}$ & $\begin{array}{r}-2594 \\
-2574 \\
3044 \\
2357\end{array}$ & $\begin{array}{r}64 \\
28 \\
40 \\
118\end{array}$ & $\begin{array}{r}-2658 \\
-2602 \\
3004 \\
2201\end{array}$ & $\begin{array}{r}55 \\
0 \\
30 \\
0\end{array}$ & $\begin{array}{r}-2764 \\
-2661 \\
2731 \\
2027\end{array}$ & $\begin{array}{r}15 \\
15 \\
107 \\
110\end{array}$ & $\begin{array}{r}-3308 \\
-3305 \\
\\
2624 \\
1896\end{array}$ & $\begin{array}{l}215 \\
192\end{array}$ & $\begin{array}{l}10586 \\
10741\end{array}$ & $\begin{array}{l}210 \\
225\end{array}$ & $\mathbf{T}$ \\
\hline & & & & $\begin{array}{l}3219 \\
3329\end{array}$ & 40 & $\begin{array}{l}2723 \\
3184 \\
2996 \\
2835\end{array}$ & $\begin{array}{l}18 \\
10 \\
10 \\
31\end{array}$ & $\begin{array}{l}2656 \\
3174 \\
2986 \\
2763\end{array}$ & $\begin{array}{l}35 \\
42 \\
47 \\
50\end{array}$ & $\begin{array}{l}2430 \\
2964 \\
2765 \\
2607 \\
2700\end{array}$ & $\begin{array}{l}59 \\
70 \\
71 \\
76 \\
87\end{array}$ & $\begin{array}{l}2371 \\
2894 \\
2694 \\
2531 \\
2613\end{array}$ & $\begin{array}{l}125 \\
100 \\
110 \\
110 \\
145\end{array}$ & $\begin{array}{l}1993 \\
2584 \\
2331 \\
2212 \\
2332\end{array}$ & $\begin{array}{r}130 \\
130 \\
98 \\
140 \\
45\end{array}$ & $\begin{array}{l}1863 \\
2454 \\
2233 \\
2072 \\
2256\end{array}$ & $\begin{array}{l}70 \\
30 \\
20 \\
30 \\
60\end{array}$ & $\begin{array}{l}1611 \\
2206 \\
2005 \\
1857 \\
2005\end{array}$ & $\begin{array}{r}103 \\
74 \\
90 \\
115 \\
70\end{array}$ & $\begin{array}{l}1508 \\
2084 \\
1889 \\
1742 \\
1894\end{array}$ & $\begin{array}{l}36 \\
40 \\
45 \\
40\end{array}$ & $\begin{array}{l}2620 \\
2786\end{array}$ & $\begin{array}{l}90 \\
91\end{array}$ & $\begin{array}{l}p \\
P \\
M \\
P \\
P\end{array}$ \\
\hline $\begin{array}{l}-150 \\
-165 \\
-334 \\
-461\end{array}$ & $\begin{array}{l}35 \\
35 \\
25 \\
35\end{array}$ & $\begin{array}{l}-248 \\
-270 \\
-441 \\
-568\end{array}$ & $\begin{array}{l}0 \\
0 \\
0 \\
0\end{array}$ & $\begin{array}{l}-491 \\
-535 \\
-723 \\
-890\end{array}$ & $\begin{array}{l}60 \\
60 \\
65 \\
40\end{array}$ & $\begin{array}{r}2620 \\
-952 \\
-1008 \\
-1181 \\
-1327\end{array}$ & $\begin{array}{l}25 \\
30 \\
26 \\
18 \\
18\end{array}$ & $\begin{array}{r}2590 \\
-985 \\
-1034 \\
-1199 \\
-1345\end{array}$ & $\begin{array}{r}83 \\
78 \\
80 \\
96 \\
118\end{array}$ & $\begin{array}{r}2405 \\
-1155 \\
-1211 \\
-1384 \\
-1548\end{array}$ & $\begin{array}{l}91 \\
82 \\
80 \\
89 \\
83\end{array}$ & $\begin{array}{r}2314 \\
-1237 \\
-1291 \\
-1473 \\
-1631\end{array}$ & $\begin{array}{r}86 \\
105 \\
60 \\
50 \\
32\end{array}$ & $\begin{array}{r}2032 \\
-1467 \\
-1544 \\
-1723 \\
-1837\end{array}$ & $\begin{array}{r}83 \\
74 \\
75 \\
80 \\
105\end{array}$ & $\begin{array}{r}1949 \\
-1541 \\
-1619 \\
-1803 \\
-1942\end{array}$ & $\begin{array}{r}60 \\
50 \\
70 \\
60 \\
0\end{array}$ & $\begin{array}{r}1712 \\
-1765 \\
-1833 \\
-2019 \\
-2108\end{array}$ & $\begin{array}{l}86 \\
80 \\
75 \\
50 \\
30\end{array}$ & $\begin{array}{r}1560 \\
-1895 \\
-2009 \\
-2197 \\
-2367\end{array}$ & $\begin{array}{l}55 \\
15 \\
30 \\
25 \\
40\end{array}$ & $\begin{array}{l}2160 \\
7100 \\
5420 \\
6492 \\
6155\end{array}$ & $\begin{array}{l}150 \\
140 \\
135\end{array}$ & $\begin{array}{l}P \\
M \\
M \\
M \\
M\end{array}$ \\
\hline $\begin{array}{r}-75 \\
260 \\
-913\end{array}$ & $\begin{array}{l}25 \\
20 \\
10\end{array}$ & $\begin{array}{r}-161 \\
175 \\
-1018\end{array}$ & $\begin{array}{l}0 \\
0 \\
0\end{array}$ & $\begin{array}{r}-500 \\
-195 \\
-1397\end{array}$ & $\begin{array}{l}35 \\
44 \\
20\end{array}$ & $\begin{array}{r}-600 \\
-906 \\
-603 \\
-1791\end{array}$ & $\begin{array}{r}5 \\
16 \\
17 \\
14\end{array}$ & $\begin{array}{r}-619 \\
-925 \\
-620 \\
-1805\end{array}$ & $\begin{array}{l}113 \\
113 \\
126 \\
115\end{array}$ & $\begin{array}{r}-812 \\
-1127 \\
-833 \\
-2018\end{array}$ & $\begin{array}{l}80 \\
80 \\
78 \\
82\end{array}$ & $\begin{array}{r}-892 \\
-1210 \\
-911 \\
-2100\end{array}$ & $\begin{array}{l}70 \\
50 \\
70 \\
50\end{array}$ & $\begin{array}{l}-1199 \\
-1480 \\
-1145 \\
-2342\end{array}$ & $\begin{array}{r}68 \\
78 \\
102 \\
80\end{array}$ & $\begin{array}{l}-1267 \\
-1558 \\
-1247 \\
-2422\end{array}$ & $\begin{array}{l}20 \\
30 \\
20\end{array}$ & $\begin{array}{l}-1371 \\
-1680 \\
-1366 \\
-2507\end{array}$ & $\begin{array}{l}40 \\
30 \\
30 \\
35\end{array}$ & $\begin{array}{l}-1626 \\
-1943 \\
-1663 \\
-2827\end{array}$ & $\begin{array}{l}45 \\
40 \\
40\end{array}$ & $\begin{array}{l}5344 \\
5314 \\
8584\end{array}$ & $\begin{array}{l}131 \\
170\end{array}$ & $\begin{array}{l}M \\
P \\
M\end{array}$ \\
\hline $\begin{array}{l}-1002 \\
-1540\end{array}$ & 35 & $\begin{array}{l}-1070 \\
-1617\end{array}$ & 0 & $\begin{array}{l}-1445 \\
-1939\end{array}$ & $\begin{array}{l}28 \\
23\end{array}$ & $\begin{array}{l}-1684 \\
-1706 \\
-2220 \\
-2178 \\
-1720\end{array}$ & $\begin{array}{r}5 \\
5 \\
30 \\
5 \\
10\end{array}$ & $\begin{array}{l}-1734 \\
-1739 \\
-2268 \\
-2211 \\
-1760\end{array}$ & $\begin{array}{r}111 \\
106 \\
72 \\
100 \\
65\end{array}$ & $\begin{array}{l}-2022 \\
-2012 \\
-2483 \\
-2434 \\
-2005\end{array}$ & $\begin{array}{r}81 \\
78 \\
57 \\
70 \\
110\end{array}$ & $\begin{array}{l}-2103 \\
-2090 \\
-2540 \\
-2544 \\
-2135\end{array}$ & $\begin{array}{l}50 \\
40 \\
40 \\
30 \\
10\end{array}$ & $\begin{array}{l}-2312 \\
-2303 \\
-2664 \\
-2207\end{array}$ & $\begin{array}{r}107 \\
90 \\
35 \\
22\end{array}$ & $\begin{array}{l}-2419 \\
-2393 \\
-2699 \\
-2229\end{array}$ & 65 & $\begin{array}{l}-2509 \\
-2480\end{array}$ & $\begin{array}{l}25 \\
30\end{array}$ & $\begin{array}{l}-2816 \\
-2667\end{array}$ & $\begin{array}{r}145 \\
45\end{array}$ & $\begin{array}{l}7707 \\
6089\end{array}$ & $\begin{array}{l}160 \\
131\end{array}$ & $P$ \\
\hline \multirow[t]{3}{*}{$\begin{array}{r}-964 \\
-1543 \\
-1390\end{array}$} & $\begin{array}{l}35 \\
25 \\
50\end{array}$ & $\begin{array}{l}-1033 \\
-1647 \\
-1515\end{array}$ & $\begin{array}{l}0 \\
0 \\
0\end{array}$ & $\begin{array}{l}-1415 \\
-2021 \\
-1884\end{array}$ & $\begin{array}{l}17 \\
15 \\
10\end{array}$ & $\begin{array}{r}-1632 \\
-2208 \\
-2060 \\
\\
3554\end{array}$ & $\begin{array}{l}15 \\
20 \\
25 \\
35\end{array}$ & $\begin{array}{r}-1671 \\
-2244 \\
-2118 \\
3456\end{array}$ & $\begin{array}{l}65 \\
60 \\
60 \\
36\end{array}$ & $\begin{array}{r}-1911 \\
-2525 \\
-2387 \\
\\
3248\end{array}$ & $\begin{array}{r}119 \\
59 \\
54 \\
48\end{array}$ & $\begin{array}{r}-2045 \\
-2584 \\
-2441 \\
3196\end{array}$ & $\begin{array}{r}0 \\
80 \\
107 \\
130\end{array}$ & $\begin{array}{r}-2114 \\
-2731 \\
-2548 \\
\\
2794\end{array}$ & $\begin{array}{r}62 \\
47 \\
44 \\
116\end{array}$ & $\begin{array}{r}-2176 \\
-2778 \\
-2593 \\
4164 \\
2678\end{array}$ & $\begin{array}{r}35 \\
0 \\
20 \\
70 \\
85\end{array}$ & $\begin{array}{r}-2254 \\
-2884 \\
-2705 \\
3939 \\
2422\end{array}$ & $\begin{array}{l}15 \\
20 \\
15 \\
55 \\
96\end{array}$ & $\begin{array}{r}-2953 \\
-3498 \\
\\
3884 \\
2326\end{array}$ & $\begin{array}{r}195 \\
100 \\
0 \\
30\end{array}$ & $\begin{array}{r}9687 \\
10813 \\
6294 \\
1594 \\
2498\end{array}$ & $\begin{array}{r}200 \\
189 \\
138 \\
65 \\
78\end{array}$ & $\begin{array}{l}T \\
J \\
J \\
p \\
P\end{array}$ \\
\hline & & & & & & $\begin{array}{l}3<80 \\
3105 \\
3468\end{array}$ & $\begin{array}{r}30 \\
0 \\
11\end{array}$ & $\begin{array}{l}3211 \\
3075 \\
3457\end{array}$ & $\begin{array}{l}36 \\
38 \\
43\end{array}$ & $\begin{array}{l}3013 \\
2764 \\
2857 \\
3269\end{array}$ & $\begin{array}{l}47 \\
71 \\
62 \\
58\end{array}$ & $\begin{array}{l}2956 \\
2693 \\
2795 \\
3201\end{array}$ & $\begin{array}{r}186 \\
75 \\
110 \\
110\end{array}$ & $\begin{array}{l}2547 \\
2308 \\
2396 \\
2808 \\
3630\end{array}$ & $\begin{array}{r}136 \\
96 \\
85 \\
114 \\
58\end{array}$ & $\begin{array}{l}2411 \\
2212 \\
2297 \\
2684 \\
3572\end{array}$ & $\begin{array}{l}55 \\
30 \\
30 \\
30\end{array}$ & $\begin{array}{l}2146 \\
1904 \\
2033 \\
2412 \\
3268\end{array}$ & $\begin{array}{r}80 \\
115 \\
130 \\
152 \\
90\end{array}$ & $\begin{array}{l}2022 \\
1789 \\
1875 \\
2250 \\
3162\end{array}$ & 50 & 2592 & 83 & $\begin{array}{l}P \\
P\end{array}$ \\
\hline & & & & & & & & & $\begin{array}{l}59 \\
63 \\
68 \\
74\end{array}$ & $\begin{array}{r}3236 \\
2695 \\
2262 \\
930\end{array}$ & $\begin{array}{l}89 \\
77 \\
86 \\
91\end{array}$ & $\begin{array}{r}3147 \\
2618 \\
2176 \\
839\end{array}$ & $\begin{array}{l}110 \\
160 \\
135 \\
125\end{array}$ & $\begin{array}{r}2837 \\
2293 \\
1870 \\
507\end{array}$ & $\begin{array}{r}98 \\
80 \\
127 \\
108\end{array}$ & $\begin{array}{r}2739 \\
2213 \\
1743 \\
399\end{array}$ & $\begin{array}{l}90 \\
120 \\
110 \\
115\end{array}$ & $\begin{array}{r}2477 \\
1918 \\
1480 \\
176\end{array}$ & $\begin{array}{l}55 \\
80 \\
90 \\
75\end{array}$ & & & $\begin{array}{l}2505 \\
2269 \\
2727 \\
3807\end{array}$ & $\begin{array}{r}95 \\
93 \\
94 \\
118\end{array}$ & $\begin{array}{l}\mathrm{J} \\
\mathrm{J} \\
\mathrm{J} \\
\mathrm{J}\end{array}$ \\
\hline 2219 & 22 & 2129 & 0 & 1880 & 75 & 1494 & 24 & 1470 & 85 & 1294 & 86 & 1208 & 83 & 941 & 25 & 910 & 80 & & & & & 2336 & & 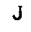 \\
\hline
\end{tabular}


NTERVAL IOENIIFICATION

\begin{tabular}{|c|c|c|c|c|c|c|c|c|c|c|c|c|c|c|c|c|c|c|c|c|c|c|}
\hline LOCAL NUMBER & $\begin{array}{l}\text { WELL } \\
\text { NAME }\end{array}$ & $\begin{array}{l}K B \\
F T\end{array}$ & $\begin{array}{l}\text { LAND } \\
\text { ELEV } \\
\text { FI }\end{array}$ & $\begin{array}{l}--A-- \\
\text { SAND } \\
\text { FT }\end{array}$ & $\begin{array}{l}-\infty-\theta \\
\text { IOP } \\
\text { ELEV } \\
\text { FT }\end{array}$ & $\begin{array}{l}\text { SAND } \\
\text { FT }\end{array}$ & $\begin{array}{l}\cdots \text { TUP } \\
\text { ELEV } \\
\text { FT }\end{array}$ & $\begin{array}{l}\text { SAND } \\
\text { FT }\end{array}$ & $\begin{array}{l}\text { TOP } \\
\text { ELEV } \\
\text { FT }\end{array}$ & $\begin{array}{l}\text { SAND } \\
\text { FT }\end{array}$ & $\begin{array}{l}\text { TOP } \\
\text { ELEV } \\
\text { FT }\end{array}$ & $\begin{array}{c}\text { SAND } \\
\text { FT }\end{array}$ & $\begin{array}{l}\text { IOP } \\
\text { ELEV } \\
\text { FT }\end{array}$ & SAND & $\begin{array}{l}\text { TOP } \\
\text { ELEV } \\
F T\end{array}$ & $\begin{array}{l}\text { SAND } \\
\text { FT }\end{array}$ & $\begin{array}{l}-\cdots-H \\
\text { TOP } \\
\text { ELEV } \\
\text { FT }\end{array}$ & $\begin{array}{l}\text { SAND } \\
\text { FT }\end{array}$ & $\begin{array}{c}\text { TUP } \\
\text { ELEV } \\
F T\end{array}$ & $\begin{array}{l}\text { IDAN } \\
\text { SAT }\end{array}$ & $\begin{array}{l}\text { TUP } \\
\text { ELEV } \\
\text { FT }\end{array}$ & $\begin{array}{l}\text { J SAND } \\
\text { FI }\end{array}$ \\
\hline 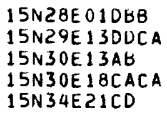 & $\begin{array}{l}1 \text { UCLI } \\
\text { NORR-1 } \\
1 \text { PETER } \\
18-11 F \\
824-21\end{array}$ & $\begin{array}{l}12 \\
09 \\
07 \\
11\end{array}$ & $\begin{array}{l}2887 \\
2843 \\
2999 \\
2710 \\
3426\end{array}$ & & & & & & & & & & 2929 & 0 & 1994 & 130 & 1582 & 0 & 1340 & $\varsigma_{0}$ & 843 & 0 \\
\hline $\begin{array}{l}\text { 15N3SEZ1CABD } \\
15 N 37 \text { E3 } 3 A A C A \\
15 N 39 E 13 B 8 \\
15 N 40 E 2100 \\
15 N 50 E 27 C C\end{array}$ & $\begin{array}{l}\text { N23-21 } \\
\text { NP33-1 } \\
\text { NCT } 3-1 \\
\text { KNCT }-2 \\
127-15\end{array}$ & $\begin{array}{l}11 \\
11 \\
12 \\
12 \\
10\end{array}$ & $\begin{array}{l}3399 \\
3128 \\
2925 \\
2743 \\
2704\end{array}$ & 300 & 1990 & 0 & 1918 & 66 & 1764 & 30 & 1555 & 118 & $\begin{array}{l}2300 \\
1064\end{array}$ & $\begin{array}{l}0 \\
0\end{array}$ & $\begin{array}{l}1920 \\
1988 \\
1802 \\
1301 \\
143\end{array}$ & $\begin{array}{r}133 \\
95 \\
70 \\
60 \\
20\end{array}$ & $\begin{array}{r}1585 \\
1675 \\
1589 \\
1060 \\
10\end{array}$ & $\begin{array}{l}0 \\
0 \\
0 \\
0 \\
0\end{array}$ & $\begin{array}{r}1303 \\
1348 \\
1266 \\
792 \\
-255\end{array}$ & $\begin{array}{r}55 \\
50 \\
30 \\
25 \\
0\end{array}$ & $\begin{array}{r}838 \\
851 \\
733 \\
230 \\
-1174\end{array}$ & $\begin{array}{l}0 \\
0 \\
0 \\
0 \\
0\end{array}$ \\
\hline $\begin{array}{l}15 N 52 E 270 C \\
15 N 53 E 22 C \\
15 N 54 E O 3 D O B D \\
15 N 55 E 250 A C C \\
14 N 14 E 0 O A D D B\end{array}$ & $\begin{array}{l}1-16 N P \\
H 11-22 \\
\text { GU44-3 } \\
\text { BN1-25 } \\
\text { PORT-1 }\end{array}$ & $\begin{array}{l}06 \\
10 \\
15 \\
12 \\
09\end{array}$ & $\begin{array}{l}2851 \\
2534 \\
2480 \\
2361 \\
4249\end{array}$ & $\begin{array}{l}230 \\
190\end{array}$ & $\begin{array}{l}2105 \\
2037\end{array}$ & $\begin{array}{l}10 \\
25\end{array}$ & $\begin{array}{l}2023 \\
1946\end{array}$ & $\begin{array}{l}110 \\
100\end{array}$ & $\begin{array}{l}1828 \\
1726\end{array}$ & $\begin{array}{l}25 \\
60\end{array}$ & $\begin{array}{l}1480 \\
1401\end{array}$ & $\begin{array}{l}250 \\
125\end{array}$ & $\begin{array}{r}1053 \\
990 \\
1886\end{array}$ & $\begin{array}{l}0 \\
0 \\
0\end{array}$ & $\begin{array}{r}154 \\
86 \\
1036 \\
1023\end{array}$ & $\begin{array}{r}20 \\
30 \\
15 \\
0\end{array}$ & $\begin{array}{r}21 \\
-74 \\
911 \\
918\end{array}$ & $\begin{array}{l}0 \\
0 \\
0 \\
0\end{array}$ & $\begin{array}{r}-256 \\
669 \\
688\end{array}$ & $\begin{array}{l}0 \\
0 \\
0\end{array}$ & $\begin{array}{r}-1227 \\
-385 \\
-417\end{array}$ & $\begin{array}{l}0 \\
0\end{array}$ \\
\hline 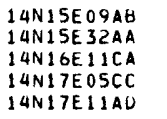 & $\begin{array}{l}\text { IBECHT } \\
41-32 \\
\text { LEE } 1 \\
\text { LONG } 1 \\
\text { ISMITH }\end{array}$ & $\begin{array}{l}10 \\
10 \\
10 \\
11 \\
10\end{array}$ & $\begin{array}{l}4074 \\
4125 \\
4167 \\
4192 \\
4365\end{array}$ & & & & & & & & & & & & & & & & & & & \\
\hline $\begin{array}{l}14 N 24 E 02 B A B D \\
14 N 25 E 23 D D \\
14 N 25 E 35 B B \\
14 N 26 E 05 A B \\
14 N 26 E 330 D\end{array}$ & $\begin{array}{l}1-A R C H \\
\text { KING } 1 \\
1-E V A N \\
386 \text { TIE } \\
16-33\end{array}$ & $\begin{array}{r}7 \\
7 \\
6 \\
5 \\
11\end{array}$ & $\begin{array}{l}3667 \\
3145 \\
3227 \\
3077 \\
3144\end{array}$ & & & & & & & & & & & & & & & & & & & \\
\hline $\begin{array}{l}14 N 27 E 04 B B \\
14 N 2 B E 0 O D D \\
14 N 2 B E 27 C A \\
14 N 29 E 02 A C C D \\
14 N 29 E 2 B D A D\end{array}$ & $\begin{array}{l}1-B R A T \\
B D H N 1 \\
23-27 \\
1-W I S E \\
43-28\end{array}$ & $\begin{array}{l}11 \\
10 \\
12 \\
12 \\
12\end{array}$ & $\begin{array}{l}2949 \\
2844 \\
2825 \\
3045 \\
2697\end{array}$ & & & & & & & & & & & & & & & & & & $\begin{array}{l}2780 \\
2427 \\
2520\end{array}$ & $\begin{array}{l}0 \\
0 \\
0\end{array}$ \\
\hline $\begin{array}{l}14 N 30 E 270 C D E \\
14 N 31 E 06 B C D \\
14 N 31 E 0700 D \\
14 N 31 E 21 C B A C \\
14 N 32 E 01 B B\end{array}$ & $\begin{array}{l}:-H I L L \\
0 \\
P P \\
-N P \\
-I N P R\end{array}$ & $\begin{array}{r}9 \\
5 \\
10 \\
11\end{array}$ & $\begin{array}{l}2571 \\
2751 \\
2663 \\
2809 \\
2902\end{array}$ & & & & & & & & & & & & 1852 & 140 & 1607 & 0 & 1281 & & & \\
\hline $\begin{array}{l}14 N 32 E 31 A C B D \\
14 N 33 E 03 A C A A \\
14 N 33 E 05 A A \\
14 N 34 E 02 C C \\
14 N 35 E 07 B C\end{array}$ & $\begin{array}{l}N P-1 \\
A L-A Q U \\
1 N P \\
14-2 \\
1-B A T T\end{array}$ & $\begin{array}{l}11 \\
10 \\
10 \\
12\end{array}$ & $\begin{array}{l}3034 \\
3141 \\
3194 \\
3494 \\
3422\end{array}$ & & & & & & & & & & $\begin{array}{l}2974 \\
2947 \\
2999\end{array}$ & $\begin{array}{l}0 \\
0\end{array}$ & $\begin{array}{l}1846 \\
1830 \\
1829\end{array}$ & $\begin{array}{l}150 \\
130 \\
130\end{array}$ & $\begin{array}{l}1527 \\
1486 \\
1519\end{array}$ & $\begin{array}{l}0 \\
0\end{array}$ & $\begin{array}{l}1205 \\
1258\end{array}$ & $\begin{array}{l}45 \\
40\end{array}$ & $\begin{array}{l}2032 \\
760 \\
796\end{array}$ & $\begin{array}{l}0 \\
0\end{array}$ \\
\hline $\begin{array}{l}\text { I } 4 \text { } 40 E 23 A A B C \\
14 N 44 E 350 A \\
14 N 45 E 330 A \\
14 N 46 E 26 A A B D \\
14 N 4 B E 05 B B B D\end{array}$ & $\begin{array}{l}1-A-B N \\
1-S W I F \\
1-N P R \\
1 \\
1-S A N D\end{array}$ & $\begin{array}{l}10 \\
18 \\
10 \\
16 \\
11\end{array}$ & $\begin{array}{l}2834 \\
2718 \\
2817 \\
3180 \\
3126\end{array}$ & $\begin{array}{r}50 \\
100 \\
225\end{array}$ & $\begin{array}{l}2424 \\
2250 \\
2332\end{array}$ & $\begin{array}{l}80 \\
45 \\
36\end{array}$ & $\begin{array}{l}2032 \\
2044 \\
2192\end{array}$ & $\begin{array}{l}60 \\
45 \\
55\end{array}$ & $\begin{array}{l}1900 \\
1931 \\
2089\end{array}$ & $\begin{array}{r}37 \\
90 \\
100\end{array}$ & $\begin{array}{l}1686 \\
1612 \\
1579 \\
1550\end{array}$ & $\begin{array}{l}108 \\
155 \\
115 \\
110\end{array}$ & $\begin{array}{l}1373 \\
1338 \\
1347 \\
1282\end{array}$ & $\begin{array}{l}0 \\
0 \\
0 \\
0\end{array}$ & $\begin{array}{r}1332 \\
496 \\
440 \\
425 \\
419\end{array}$ & $\begin{array}{r}60 \\
30 \\
25 \\
0 \\
15\end{array}$ & $\begin{array}{r}1084 \\
359 \\
252 \\
290 \\
312\end{array}$ & $\begin{array}{l}0 \\
0 \\
0 \\
0 \\
0\end{array}$ & $\begin{array}{r}829 \\
36 \\
-18 \\
-6 \\
-53\end{array}$ & $\begin{array}{r}27 \\
40 \\
37 \\
0 \\
0\end{array}$ & $\begin{array}{r}122 \\
-771 \\
-838 \\
-876 \\
-954\end{array}$ & $\begin{array}{l}0 \\
0 \\
0 \\
0 \\
0\end{array}$ \\
\hline $\begin{array}{l}14 N 49 E 11 C C \\
14 N 50 E 2400 \\
14 N 51 E 290 D \\
14 N 52 E 17 B C B D \\
14 N 55 E 17 A D O C\end{array}$ & $\begin{array}{l}1-11 N P \\
1=24 \\
1=29 N P \\
1-15 N P \\
N C T 9-3\end{array}$ & $\begin{array}{r}11 \\
10 \\
11 \\
7 \\
11\end{array}$ & $\begin{array}{l}2792 \\
2775 \\
2681 \\
2713 \\
2092\end{array}$ & $\begin{array}{r}250 \\
175 \\
60 \\
255\end{array}$ & $\begin{array}{l}2078 \\
2148 \\
2405 \\
1952\end{array}$ & $\begin{array}{r}0 \\
25 \\
43 \\
0\end{array}$ & $\begin{array}{l}2028 \\
2012 \\
2238 \\
1843\end{array}$ & $\begin{array}{r}62 \\
70 \\
195 \\
40\end{array}$ & $\begin{array}{l}1918 \\
1871 \\
1944 \\
1769\end{array}$ & $\begin{array}{r}54 \\
0 \\
75 \\
20\end{array}$ & $\begin{array}{l}1688 \\
1598 \\
1565 \\
1445\end{array}$ & $\begin{array}{l}191 \\
175 \\
195 \\
210\end{array}$ & $\begin{array}{l}1273 \\
1210 \\
1231 \\
1164\end{array}$ & $\begin{array}{l}0 \\
0 \\
0 \\
0\end{array}$ & $\begin{array}{r}360 \\
329 \\
336 \\
235 \\
1346\end{array}$ & $\begin{array}{r}9 \\
10 \\
77 \\
17 \\
11\end{array}$ & $\begin{array}{l}205 \\
227 \\
245 \\
136 \\
1237\end{array}$ & $\begin{array}{l}0 \\
0 \\
0 \\
0 \\
0\end{array}$ & $\begin{array}{r}-59 \\
-100 \\
-97 \\
-191 \\
1005\end{array}$ & $\begin{array}{l}0 \\
0 \\
0 \\
0 \\
0\end{array}$ & $\begin{array}{r}-969 \\
-1027 \\
-1026 \\
-1126 \\
-57\end{array}$ & $\begin{array}{l}0 \\
0 \\
0 \\
0 \\
0\end{array}$ \\
\hline
\end{tabular}

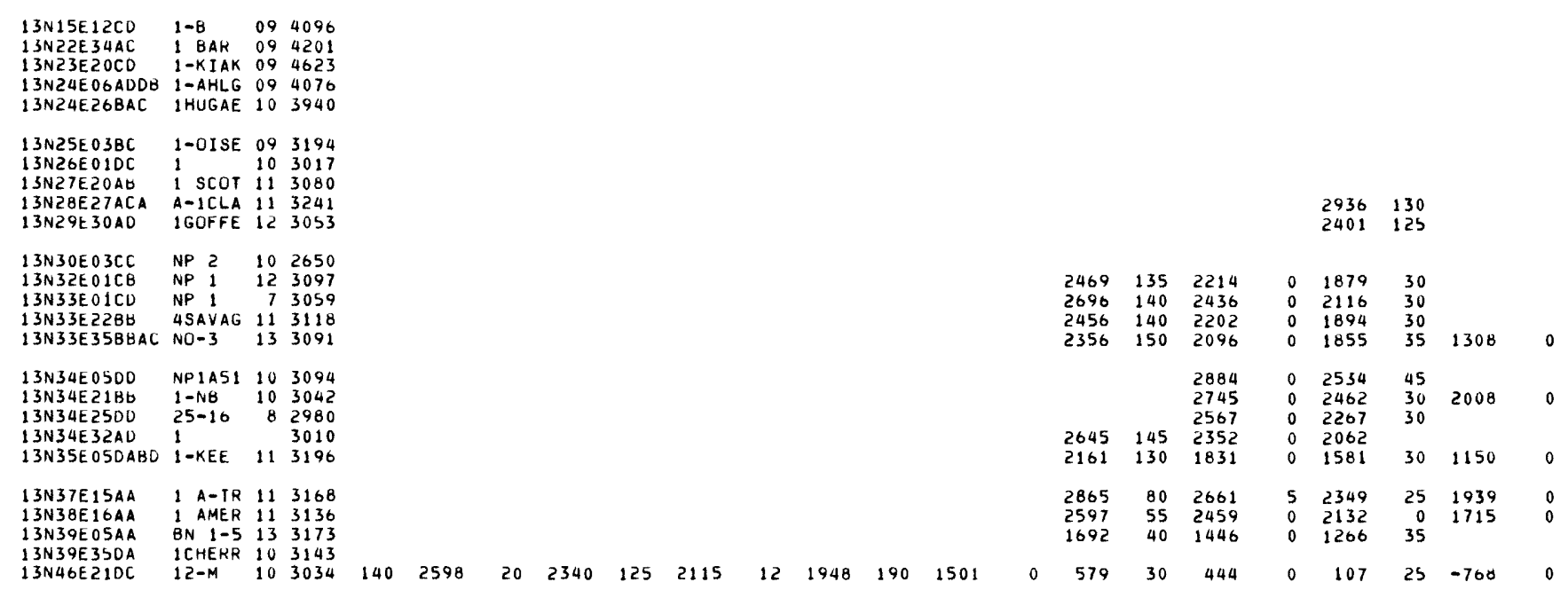




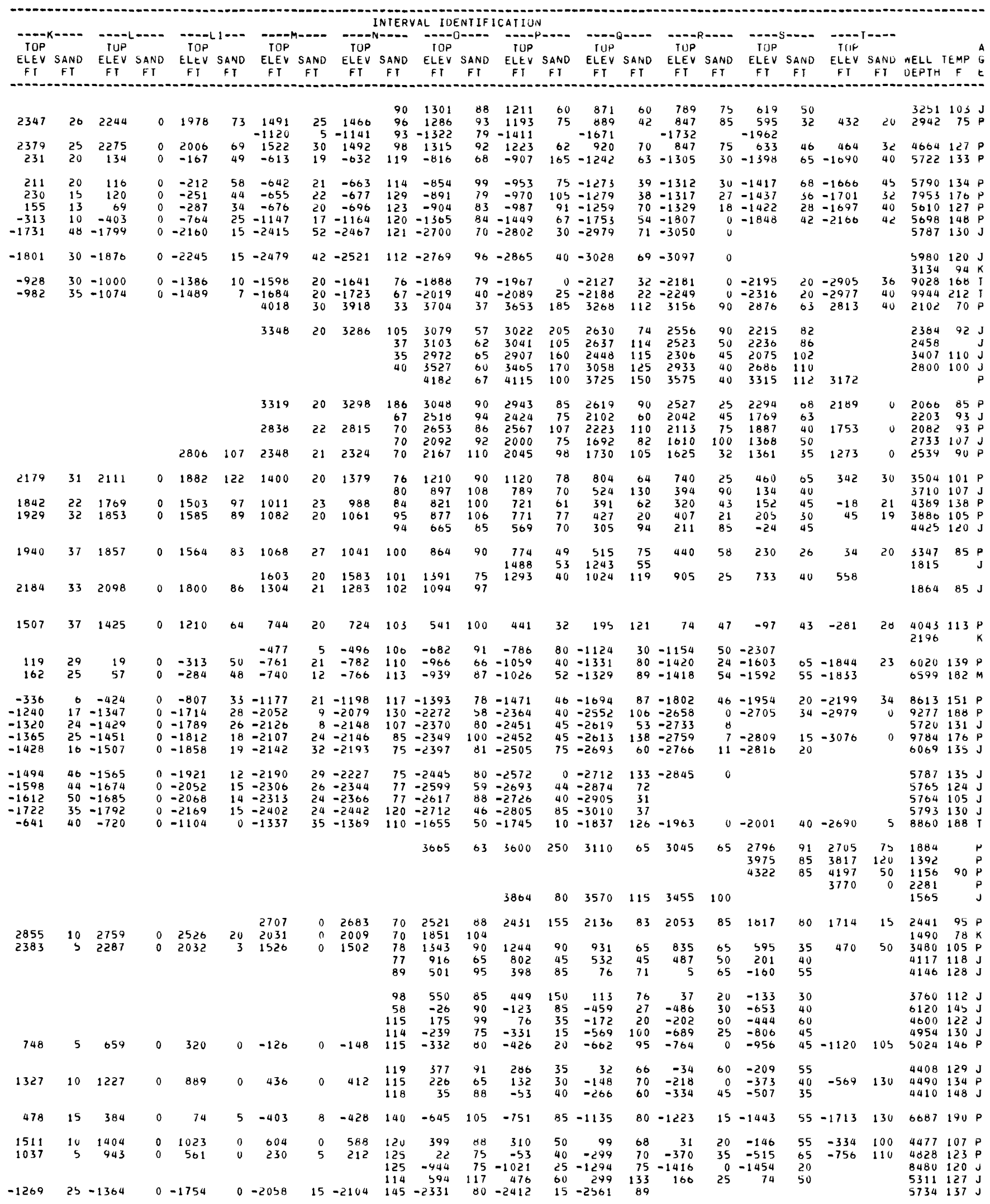




\begin{tabular}{|c|c|c|c|c|c|c|c|c|c|c|c|c|c|c|c|c|c|c|c|c|c|c|}
\hline LOCAL NUMBER & $\begin{array}{l}\text { WELL } \\
\text { NAME }\end{array}$ & $\begin{array}{l}K E \\
F T\end{array}$ & $\begin{array}{l}\text { LAND } \\
\text { ELEV } \\
\text { FT }\end{array}$ & $\begin{array}{l}\text { SAND } \\
\text { SAN }\end{array}$ & $\begin{array}{l}\text { TOP } \\
\text { ELEV } \\
\text { FT }\end{array}$ & $\begin{array}{l}\text { SAND } \\
\text { FI }\end{array}$ & $\begin{array}{l}-D-C \\
\text { TOP } \\
\text { ELEV } \\
F T\end{array}$ & $\begin{array}{l}\text { SAND } \\
\text { FT }\end{array}$ & $\begin{array}{c}\text { TOP } \\
\text { ELEV } \\
F T\end{array}$ & $\begin{array}{l}\text { SAND } \\
\text { FT }\end{array}$ & $\begin{array}{l}\text { INTERV } \\
\text { TUP } \\
\text { ELEV } \\
\text { FT }\end{array}$ & $\begin{array}{l}\text { SAL IUE } \\
\text { FT }\end{array}$ & $\begin{array}{l}\text { ENIIF I } \\
\text { TUP } \\
\text { ELEV } \\
\text { FT }\end{array}$ & $\begin{array}{l}\text { CAIIO } \\
--.-- \\
\text { SAND } \\
\text { FI }\end{array}$ & $\begin{array}{l}\text { FLEV } \\
\text { FLP }\end{array}$ & $\begin{array}{c}\text { SAND } \\
\text { F T }\end{array}$ & $\begin{array}{l}\qquad \text { TUP } \\
\text { ELEV } \\
\text { FT }\end{array}$ & $\begin{array}{c}\text { SAND } \\
\text { FT }\end{array}$ & $\begin{array}{c}\text { TUP } \\
\text { ELEV } \\
\text { FT }\end{array}$ & $\begin{array}{l}\text { SAND } \\
\text { FT }\end{array}$ & $\begin{array}{l}\text { FT } \\
\text { IUP } \\
---J \\
\text { FLE }\end{array}$ & $\begin{array}{c}\text { SAND } \\
\text { FT }\end{array}$ \\
\hline $\begin{array}{l}13 N 47 E 23 A A \\
13 N 49 E 33 A A \\
13 N 50 E 27 C C A C \\
13 N 52 E 11 B B \\
13 N 55 E 1300 D B\end{array}$ & $\begin{array}{l}13-M \\
N P 1-33 \\
1-27 \\
1-11 \\
44-130\end{array}$ & $\begin{array}{l}10 \\
11 \\
11 \\
15\end{array}$ & $\begin{array}{l}2923 \\
2592 \\
2547 \\
2597 \\
2457\end{array}$ & 145 & $\begin{array}{l}2434 \\
2410 \\
2359\end{array}$ & $\begin{array}{l}22 \\
30 \\
10\end{array}$ & $\begin{array}{l}2251 \\
2255 \\
2239\end{array}$ & $\begin{array}{r}115 \\
95 \\
120\end{array}$ & $\begin{array}{l}2069 \\
2020 \\
1982\end{array}$ & $\begin{array}{r}20 \\
105 \\
5\end{array}$ & $\begin{array}{l}1747 \\
1707 \\
1714\end{array}$ & $\begin{array}{l}150 \\
140 \\
110\end{array}$ & $\begin{array}{l}1461 \\
1408 \\
1426 \\
1266\end{array}$ & $\begin{array}{l}0 \\
0 \\
0 \\
0\end{array}$ & $\begin{array}{l}568 \\
531 \\
524 \\
397\end{array}$ & $\begin{array}{l}10 \\
10 \\
25 \\
25\end{array}$ & $\begin{array}{l}426 \\
423 \\
393 \\
252\end{array}$ & $\begin{array}{l}0 \\
0 \\
0 \\
0\end{array}$ & $\begin{array}{r}96 \\
80 \\
50 \\
-58 \\
1155\end{array}$ & $\begin{array}{r}25 \\
0 \\
0\end{array}$ & $\begin{array}{r}-842 \\
-837 \\
-896 \\
73\end{array}$ & $\begin{array}{l}0 \\
0 \\
0\end{array}$ \\
\hline $\begin{array}{l}13 N 56 E 05 A B \\
13 N 59 E 20 B A C A \\
13 N 60 E 29 C C \\
1 \text { 2N23E2 } 2 \text { CCDBU } \\
1 \text { 2N24E36AA }\end{array}$ & $\begin{array}{l}\text { I-DAWS } \\
\text { ROJIC1 } \\
\text { MISKE1 } \\
24-24 \\
41-30\end{array}$ & $\begin{array}{l}0 \\
12 \\
12 \\
09 \\
10\end{array}$ & $\begin{array}{l}2521 \\
2964 \\
2840 \\
3898 \\
3814\end{array}$ & & & & & & & & 2158 & 140 & $\begin{array}{l}2162 \\
1893\end{array}$ & $\begin{array}{l}0 \\
0\end{array}$ & & & $\begin{array}{r}1026 \\
862\end{array}$ & $\begin{array}{l}0 \\
0\end{array}$ & $\begin{array}{r}1035 \\
588 \\
432\end{array}$ & $\begin{array}{l}0 \\
0 \\
0\end{array}$ & $\begin{array}{r}-43 \\
-458 \\
-601\end{array}$ & $\begin{array}{l}0 \\
0 \\
0\end{array}$ \\
\hline $\begin{array}{l}12 N 25 E 3400 D B \\
12 N 26 E 32 A O C \\
12 N 28 E 14 C B \\
12 N 29 E 1700 \\
12 N 29 E 350 D A C\end{array}$ & $\begin{array}{l}34-16 \\
\text { HARM } 1 \\
\text { GOFF } 2 \\
\text { NP } 1 \\
\text { NPRR } 1\end{array}$ & $\begin{array}{r}10 \\
7 \\
10 \\
12 \\
6\end{array}$ & $\begin{array}{l}3706 \\
3599 \\
3176 \\
2972 \\
3090\end{array}$ & & & & & & & & & & & & & & $\begin{array}{l}3048 \\
2924\end{array}$ & $\begin{array}{l}0 \\
0\end{array}$ & $\begin{array}{l}2616 \\
2289 \\
2575\end{array}$ & $\begin{array}{r}125 \\
125 \\
80\end{array}$ & $\begin{array}{l}3398 \\
2119 \\
2012\end{array}$ & $\begin{array}{l}20 \\
30 \\
15\end{array}$ \\
\hline $\begin{array}{l}12 N 30 E 27 B B A C \\
12 N 31 E 320 C \\
12 N 32 E 310 B \\
12 N 32 E 320 C \\
12 N 33 E 30 C D B\end{array}$ & $\begin{array}{l}\text { HALL I } \\
1 \text { COLLI } \\
33-31 \\
1 \text { COLLI } \\
30-14\end{array}$ & $\begin{array}{r}11 \\
7 \\
8 \\
8\end{array}$ & $\begin{array}{l}2738 \\
2774 \\
3064 \\
2781 \\
3163\end{array}$ & & & & & & & & & & $\begin{array}{l}2802 \\
2886\end{array}$ & $\begin{array}{l}0 \\
0\end{array}$ & $\begin{array}{l}2297 \\
1812 \\
1959 \\
1894\end{array}$ & $\begin{array}{l}140 \\
140 \\
140 \\
145\end{array}$ & $\begin{array}{l}2064 \\
1554 \\
1701 \\
1621\end{array}$ & $\begin{array}{l}0 \\
0 \\
0\end{array}$ & $\begin{array}{l}1700 \\
1225 \\
1321 \\
1303\end{array}$ & $\begin{array}{l}50 \\
69 \\
40\end{array}$ & $\begin{array}{r}1172 \\
697 \\
789\end{array}$ & 0 \\
\hline $\begin{array}{l}12 N 34 E 06 A A B D \\
12 N 35 E 27 C C B D \\
12 N 36 E 03 A A \\
12 N 38 E 27 A D \\
12 N 39 E 09 A A\end{array}$ & $\begin{array}{l}43-6 \\
13-27 \\
1 \quad N P \\
1 \text { NPRR } \\
\text { NP } 3\end{array}$ & $\begin{array}{r}10 \\
11 \\
7 \\
7 \\
7\end{array}$ & $\begin{array}{l}3152 \\
2904 \\
3332 \\
3105 \\
2975\end{array}$ & & & & & & & & & & & & 2332 & 112 & $\begin{array}{l}2050 \\
2553\end{array}$ & $\begin{array}{l}0 \\
0\end{array}$ & $\begin{array}{l}1761 \\
2275 \\
2781\end{array}$ & $\begin{array}{l}40 \\
40 \\
45\end{array}$ & $\begin{array}{l}1232 \\
1673\end{array}$ & $\begin{array}{l}0 \\
0\end{array}$ \\
\hline $\begin{array}{l}12 N 40 E 02 A B \\
12 N 40 E 07 C C \\
12 N 41 E 25 B O D B \\
12 N 45 E 090 D \\
12 N 46 E 13 D B\end{array}$ & $\begin{array}{l}31-2 \\
1-7 \\
N P 1 \\
N P 9-M \\
\text { NP } 10 M\end{array}$ & $\begin{array}{l}13 \\
10 \\
12 \\
10 \\
10\end{array}$ & $\begin{array}{l}3115 \\
3168 \\
3082 \\
3155 \\
2958\end{array}$ & 150 & 2648 & 40 & 2518 & 130 & $\begin{array}{l}2607 \\
2346\end{array}$ & $\begin{array}{l}130 \\
115\end{array}$ & $\begin{array}{l}1920 \\
1790\end{array}$ & $\begin{array}{l}183 \\
140\end{array}$ & $\begin{array}{l}1966 \\
154 \mathrm{~B} \\
1548\end{array}$ & $\begin{array}{l}0 \\
0 \\
0\end{array}$ & $\begin{array}{r}2728 \\
1018 \\
657 \\
655\end{array}$ & $\begin{array}{l}60 \\
35 \\
27 \\
30\end{array}$ & $\begin{array}{r}2408 \\
886 \\
489 \\
512\end{array}$ & $\begin{array}{l}0 \\
0 \\
0\end{array}$ & $\begin{array}{r}1400 \\
2 B 08 \\
492 \\
171 \\
170\end{array}$ & $\begin{array}{l}25 \\
30 \\
20 \\
25 \\
20\end{array}$ & $\begin{array}{l}-240 \\
-678 \\
-725\end{array}$ & $\begin{array}{l}0 \\
0 \\
0\end{array}$ \\
\hline $\begin{array}{l}12 N 47 E 23 A A \\
12 N 48 E 14 D C \\
12 N 49 E 33 C C C A \\
12 N 53 E 33 A B D B \\
12 N 56 E 12 C D B D\end{array}$ & $\begin{array}{l}\text { NP } 11 M \\
1-24 \\
O H I O 1 \\
N P \quad 1 \\
24-12 B\end{array}$ & $\begin{array}{l}10 \\
18 \\
13 \\
12 \\
12\end{array}$ & $\begin{array}{l}2707 \\
2694 \\
2509 \\
2645 \\
2567\end{array}$ & & & & 2390 & 145 & 2135 & 70 & $\begin{array}{l}1792 \\
1868 \\
1699\end{array}$ & $\begin{array}{r}120 \\
183 \\
85\end{array}$ & $\begin{array}{l}1550 \\
1602 \\
1290\end{array}$ & $\begin{array}{l}0 \\
0\end{array}$ & $\begin{array}{l}642 \\
702 \\
420\end{array}$ & $\begin{array}{l}27 \\
40 \\
20\end{array}$ & $\begin{array}{l}485 \\
552 \\
281\end{array}$ & $\begin{array}{l}0 \\
0\end{array}$ & $\begin{array}{r}154 \\
214 \\
-3 \\
1297\end{array}$ & $\begin{array}{r}20 \\
0 \\
0\end{array}$ & $\begin{array}{l}-771 \\
-726 \\
-911 \\
231\end{array}$ & $\begin{array}{l}0 \\
0 \\
0\end{array}$ \\
\hline $\begin{array}{l}12 N 57 E 29 C C B \\
12 N 57 E 29 C C B D \\
12 N 60 E 34 A C \\
11 N 21 E 34 D 0 \\
11 N 22 E 22 A C\end{array}$ & $\begin{array}{l}14-29 \\
14-29 R \\
1 B E G G A \\
\text { JOHN } 1 \\
J-J 1\end{array}$ & $\begin{array}{l}12 \\
12 \\
15 \\
10 \\
07\end{array}$ & $\begin{array}{l}2577 \\
2584 \\
3119 \\
4520 \\
4115\end{array}$ & & & & & & & & & & & & & & & & 1414 & 0 & 371 & 0 \\
\hline $\begin{array}{l}11 \mathrm{~N} 23 E 18 C C D A \\
11 \mathrm{~N} 24 E 16 C D \\
11 \mathrm{~N} 25 E 23 B A C A \\
11 \mathrm{~N} 26 E 25 B C C A \\
11 \text { N27E } 130 A\end{array}$ & $\begin{array}{l}\text { GOVT } 1 \\
\text { ST } 1 \\
3-23 \\
\text { E } 5-25 \\
\text { NP } 1-13\end{array}$ & $\begin{array}{l}05 \\
10 \\
10 \\
12 \\
10\end{array}$ & $\begin{array}{l}4005 \\
4047 \\
3858 \\
3419 \\
3354\end{array}$ & & & & & & & & & & & & & & & & 3131 & 150 & $\begin{array}{l}3438 \\
2664\end{array}$ & $\begin{array}{l}0 \\
0\end{array}$ \\
\hline 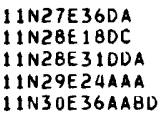 & $\begin{array}{l}43-36 \\
1 K L U Z \\
\text { BN } 1 \\
S 1-24 \\
\text { ST } 2\end{array}$ & $\begin{array}{l}10 \\
10 \\
10 \\
11 \\
12\end{array}$ & $\begin{array}{l}3311 \\
3258 \\
3352 \\
2891 \\
2944\end{array}$ & & & & & & & & & & & & 2676 & 120 & 2406 & 0 & $\begin{array}{l}3002 \\
2942 \\
2576 \\
2044\end{array}$ & $\begin{array}{r}150 \\
150 \\
95 \\
75\end{array}$ & $\begin{array}{l}2498 \\
2425 \\
2036 \\
1510\end{array}$ & $\begin{array}{l}0 \\
0 \\
0 \\
0\end{array}$ \\
\hline 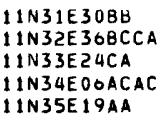 & $\begin{array}{l}\text { OSTR } 1 \\
\text { S } 5-36 \\
\text { HAVC } 1 \\
\text { H } 32-6 \\
\text { SAV } 1\end{array}$ & $\begin{array}{l}07 \\
09 \\
12 \\
09 \\
07\end{array}$ & $\begin{array}{l}3015 \\
3222 \\
3369 \\
3265 \\
3040\end{array}$ & & & & & & & & & & & & $\begin{array}{l}2855 \\
2381 \\
1841 \\
1971 \\
2471\end{array}$ & $\begin{array}{l}110 \\
160 \\
160 \\
150 \\
155\end{array}$ & $\begin{array}{l}2558 \\
2111 \\
1529 \\
1654 \\
2185\end{array}$ & $\begin{array}{l}0 \\
0 \\
0 \\
0 \\
0\end{array}$ & $\begin{array}{l}2191 \\
1819 \\
1236 \\
1393 \\
1915\end{array}$ & $\begin{array}{l}75 \\
30 \\
40 \\
40 \\
30\end{array}$ & $\begin{array}{r}1670 \\
1250 \\
678 \\
841 \\
1320\end{array}$ & $\begin{array}{l}0 \\
0 \\
0 \\
0 \\
0\end{array}$ \\
\hline $\begin{array}{l}11 \text { 1N6E32AD } \\
11 N 38 E 09 A A \\
11 N 39 E 030 D A C \\
11 \text { 1N42E34CB } \\
11 \text { N3EO1BB }\end{array}$ & $\begin{array}{ll}\text { ICMERR } \\
\text { BN } 1 \\
\text { NP } & 1 \\
1 & \\
\text { NP } & 1\end{array}$ & $\begin{array}{l}11 \\
10 \\
12 \\
10 \\
11\end{array}$ & $\begin{array}{l}2852 \\
3014 \\
2848 \\
2872 \\
2856\end{array}$ & & & & 2489 & 130 & 2167 & 50 & 1932 & 125 & 1679 & 0 & 802 & 30 & 572 & 0 & 257 & 40 & -554 & 0 \\
\hline $\begin{array}{l}11 N 44 E 27 A D \\
11 N 45 E 19 D D \\
11 N 46 E 05 D A \\
11 N 47 E 17 A A B D \\
11 N 49 E 31 B B A D\end{array}$ & $\begin{array}{l}N P R R 1 \\
N P 5-M \\
N P 7-M \\
N P 8-M \\
N P=31 N P \\
1-31 N\end{array}$ & $\begin{array}{l}11 \\
11 \\
11 \\
10 \\
12\end{array}$ & $\begin{array}{l}2820 \\
2970 \\
2931 \\
3008 \\
2574\end{array}$ & & & & $\begin{array}{l}2644 \\
2522 \\
2709\end{array}$ & $\begin{array}{r}40 \\
40 \\
100\end{array}$ & $\begin{array}{l}2594 \\
2462 \\
2425 \\
2291\end{array}$ & $\begin{array}{r}100 \\
80 \\
36 \\
25\end{array}$ & $\begin{array}{l}2136 \\
2128 \\
2036 \\
2140 \\
2103\end{array}$ & $\begin{array}{l}200 \\
260 \\
220 \\
160 \\
165\end{array}$ & $\begin{array}{l}1771 \\
1716 \\
1590 \\
1658 \\
1661\end{array}$ & $\begin{array}{l}0 \\
0 \\
0 \\
0 \\
0\end{array}$ & $\begin{array}{l}909 \\
873 \\
729 \\
847 \\
795\end{array}$ & $\begin{array}{l}35 \\
40 \\
30 \\
35 \\
30\end{array}$ & $\begin{array}{l}676 \\
681 \\
507 \\
648 \\
616\end{array}$ & $\begin{array}{l}0 \\
0 \\
0 \\
0 \\
0\end{array}$ & $\begin{array}{l}372 \\
333 \\
222 \\
330 \\
289\end{array}$ & $\begin{array}{l}30 \\
20 \\
20 \\
15 \\
20\end{array}$ & $\begin{array}{l}-502 \\
-569 \\
-667 \\
-585 \\
-682\end{array}$ & $\begin{array}{l}0 \\
0 \\
0 \\
0 \\
0\end{array}$ \\
\hline $\begin{array}{l}11 N 51 E 18 B C B D \\
11 N 52 E 11 B B B D \\
11 N 53 E 03 A A D C \\
11 \text { NSE } 26 D D D B \\
11 \text { NSTE } 16 D B A C\end{array}$ & $\begin{array}{l}\text { KEMP } 1 \\
\text { A } 1-11 \\
N P 1 \\
1-26 \\
33-16 A\end{array}$ & $\begin{array}{l}11 \\
11 \\
09 \\
10 \\
15\end{array}$ & $\begin{array}{l}2399 \\
2548 \\
2546 \\
2660 \\
2631\end{array}$ & & & & $\begin{array}{l}2311 \\
2219 \\
2253\end{array}$ & $\begin{array}{l}75 \\
70 \\
50\end{array}$ & $\begin{array}{l}2071 \\
1983 \\
1952\end{array}$ & $\begin{array}{l}35 \\
25 \\
15\end{array}$ & $\begin{array}{l}2005 \\
1826 \\
1714 \\
1647\end{array}$ & $\begin{array}{l}210 \\
185 \\
155 \\
150\end{array}$ & $\begin{array}{l}1583 \\
1453 \\
1365 \\
1337\end{array}$ & $\begin{array}{l}0 \\
0 \\
0 \\
0\end{array}$ & $\begin{array}{l}705 \\
587 \\
483 \\
480\end{array}$ & $\begin{array}{l}40 \\
25 \\
20 \\
25\end{array}$ & $\begin{array}{l}533 \\
394 \\
284 \\
262\end{array}$ & $\begin{array}{l}0 \\
0 \\
0 \\
0\end{array}$ & $\begin{array}{r}210 \\
81 \\
-13 \\
-25 \\
1386\end{array}$ & $\begin{array}{r}10 \\
10 \\
15 \\
0 \\
0\end{array}$ & $\begin{array}{r}-757 \\
-910 \\
-1012 \\
-1022 \\
353\end{array}$ & $\begin{array}{l}0 \\
0 \\
0 \\
0 \\
0\end{array}$ \\
\hline $\begin{array}{l}11 N 58 E 31 B C C A \\
11 N 59 E 29 D B D C \\
11 \text { NGOE 28BBDB } \\
1 \text { ON17E3 } 1 \text { AC } \\
1 \text { ONI BE O3ACB }\end{array}$ & $\begin{array}{l}12-31 \\
N 1-29 \\
\text { LUTT } 1 \\
B N 31 \\
\text { HA } 7-3\end{array}$ & $\begin{array}{l}11 \\
14 \\
12 \\
14 \\
12\end{array}$ & $\begin{array}{l}2666 \\
3232 \\
3163 \\
4490 \\
5165\end{array}$ & & & & 4653 & 85 & 2655 & $\begin{array}{l}60 \\
60\end{array}$ & $\begin{array}{l}2412 \\
3916\end{array}$ & $\begin{array}{l}190 \\
120\end{array}$ & $\begin{array}{l}2147 \\
3465\end{array}$ & 0 & $\begin{array}{l}1331 \\
1160 \\
2685\end{array}$ & $\begin{array}{r}10 \\
5\end{array}$ & $\begin{array}{r}1210 \\
980 \\
2794 \\
1879\end{array}$ & $\begin{array}{l}0 \\
0 \\
0 \\
0\end{array}$ & $\begin{array}{r}1414 \\
978 \\
760 \\
2346 \\
1493\end{array}$ & $\begin{array}{r}0 \\
0 \\
0 \\
220 \\
180\end{array}$ & $\begin{array}{r}353 \\
-17 \\
-245 \\
2001 \\
1093\end{array}$ & $\begin{array}{l}0 \\
0 \\
0 \\
0 \\
0\end{array}$ \\
\hline
\end{tabular}




\begin{tabular}{|c|c|c|c|c|c|c|c|c|c|c|c|c|c|c|c|c|c|c|c|c|c|c|c|c|}
\hline \multicolumn{2}{|c|}{\begin{tabular}{l} 
TOP \\
\hdashline$--K-=--$
\end{tabular}} & \multicolumn{2}{|c|}{ IOP } & \multicolumn{2}{|c|}{\begin{tabular}{c} 
TOP \\
\hdashline$-L 1-\infty-$
\end{tabular}} & \multicolumn{2}{|c|}{$-\infty-M-\infty-\infty$} & \multicolumn{2}{|c|}{$\Rightarrow i v=-2$} & \multicolumn{2}{|c|}{ TUP } & \multicolumn{2}{|c|}{$\begin{array}{l}\text { TUp } \\
-1\end{array}$} & \multicolumn{2}{|c|}{ 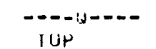 } & \multicolumn{2}{|c|}{ 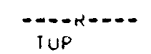 } & \multicolumn{2}{|l|}{ IUP } & \multicolumn{2}{|c|}{\begin{tabular}{l} 
tup \\
\hdashline-1
\end{tabular}} & \multirow{2}{*}{\multicolumn{2}{|c|}{$\begin{array}{l}\text { NELL IEMP } \\
\text { UEPIH F }\end{array}$}} & \\
\hline $\begin{array}{c}\text { ELEV } \\
\text { FI }\end{array}$ & $\begin{array}{l}\text { SANO } \\
\text { FT }\end{array}$ & $\begin{array}{l}\text { ELEV } \\
\text { FT }\end{array}$ & $\begin{array}{l}\text { SAND } \\
\text { FI }\end{array}$ & $\begin{array}{c}\text { ELEV } \\
\text { FI }\end{array}$ & $\begin{array}{l}\text { SAND } \\
\text { FT }\end{array}$ & $\begin{array}{l}\text { ELEV } \\
\text { FT }\end{array}$ & $\begin{array}{l}\text { SAND } \\
\text { FT }\end{array}$ & $\begin{array}{l}\text { ELEV } \\
\text { FI }\end{array}$ & $\begin{array}{l}\text { SAND } \\
\text { FT }\end{array}$ & $\begin{array}{c}\text { ELEV } \\
\text { FT }\end{array}$ & $\begin{array}{l}\text { SAND } \\
\text { FI }\end{array}$ & $\begin{array}{c}\text { ELEV } \\
\text { FI }\end{array}$ & $\begin{array}{l}\text { SANO } \\
\text { FT }\end{array}$ & $\begin{array}{c}\text { ELEV } \\
\text { FT }\end{array}$ & $\begin{array}{l}\text { SANO } \\
\text { FT }\end{array}$ & $\begin{array}{c}\text { ELEV } \\
\text { FT }\end{array}$ & $\begin{array}{l}\text { SANO } \\
\text { FT }\end{array}$ & $\begin{array}{c}\text { ELEV } \\
\text { FT }\end{array}$ & $\begin{array}{l}\text { SAND } \\
\text { FT }\end{array}$ & ELEV & $\begin{array}{l}\text { SAND } \\
\text { HT }\end{array}$ & & & \\
\hline $\begin{array}{l}-1335 \\
-1405 \\
-1477 \\
-500\end{array}$ & $\begin{array}{l}30 \\
40 \\
40\end{array}$ & $\begin{array}{l}-1410 \\
-1478 \\
-1548 \\
-602\end{array}$ & $\begin{array}{l}0 \\
0 \\
0\end{array}$ & $\begin{array}{r}-1792 \\
-1857 \\
-1939 \\
-988\end{array}$ & $\begin{array}{l}0 \\
0 \\
0\end{array}$ & $\begin{array}{l}-2093 \\
-2125 \\
-2187 \\
-2302 \\
-1234\end{array}$ & $\begin{array}{l}35 \\
25 \\
35 \\
20 \\
10\end{array}$ & $\begin{array}{l}-2138 \\
-2189 \\
-2242 \\
-2333 \\
-1280\end{array}$ & $\begin{array}{l}145 \\
125 \\
110 \\
120 \\
125\end{array}$ & $\begin{array}{l}-2366 \\
-2414 \\
-2479 \\
-2603 \\
-1554\end{array}$ & $\begin{array}{r}82 \\
90 \\
95 \\
80 \\
100\end{array}$ & $\begin{array}{l}-2450 \\
-2517 \\
-2578 \\
-2694 \\
-1678\end{array}$ & $\begin{array}{l}35 \\
50 \\
35 \\
30 \\
20\end{array}$ & $\begin{array}{l}-2634 \\
-2674 \\
-2738 \\
-2898 \\
-1795\end{array}$ & $\begin{array}{l}45 \\
30 \\
33 \\
25 \\
10\end{array}$ & $\begin{array}{l}-2713 \\
-2772 \\
-2923 \\
-1846\end{array}$ & $\begin{array}{r}35 \\
0\end{array}$ & -1881 & 20 & -2335 & 85 & $\begin{array}{l}5724 \\
5404 \\
5539\end{array}$ & $\begin{array}{l}126 \\
127 \\
128\end{array}$ & 1 \\
\hline $\begin{array}{r}-647 \\
-1046 \\
-1186\end{array}$ & $\begin{array}{l}20 \\
20 \\
15\end{array}$ & $\begin{array}{r}-743 \\
-1116 \\
-1254\end{array}$ & $\begin{array}{l}0 \\
0 \\
0\end{array}$ & $\begin{array}{r}-1136 \\
-1593 \\
-1725 \\
\\
3481\end{array}$ & $\begin{array}{l}0 \\
0 \\
0\end{array}$ & $\begin{array}{r}-1355 \\
-1766 \\
-1882 \\
\\
2952\end{array}$ & $\begin{array}{l}20 \\
30 \\
25 \\
28\end{array}$ & $\begin{array}{r}-1412 \\
-1836 \\
-1958 \\
2924\end{array}$ & $\begin{array}{r}170 \\
83 \\
75 \\
75\end{array}$ & $\begin{array}{r}-1688 \\
-2031 \\
-2241 \\
3727 \\
2761\end{array}$ & $\begin{array}{r}55 \\
70 \\
70 \\
110 \\
92\end{array}$ & $\begin{array}{r}-1825 \\
-2121 \\
-2326 \\
3617 \\
2669\end{array}$ & $\begin{array}{l}0 \\
50 \\
60 \\
85 \\
90\end{array}$ & $\begin{array}{l}3340 \\
2318\end{array}$ & $\begin{array}{l}79 \\
40\end{array}$ & $\begin{array}{r}-2012 \\
-2377 \\
3261 \\
2278\end{array}$ & $\begin{array}{r}0 \\
0 \\
65 \\
53\end{array}$ & $\begin{array}{r}-2059 \\
-2394 \\
-2576 \\
2994 \\
2085\end{array}$ & $\begin{array}{r}20 \\
20 \\
20 \\
95 \\
125\end{array}$ & $\begin{array}{r}-2737 \\
-2966 \\
-3118 \\
2836 \\
1908\end{array}$ & $\begin{array}{l}85 \\
75 \\
70 \\
20 \\
20\end{array}$ & $\begin{array}{r}9428 \\
10984 \\
10926 \\
1597 \\
2494\end{array}$ & $\begin{array}{r}195 \\
210 \\
221 \\
108 \\
94\end{array}$ & $\begin{array}{l}\text { T } \\
1 \\
1 \\
p \\
p\end{array}$ \\
\hline $\begin{array}{l}3158 \\
2714 \\
1478\end{array}$ & $\begin{array}{l}10 \\
10 \\
10\end{array}$ & $\begin{array}{l}3096 \\
2058 \\
1403\end{array}$ & $\begin{array}{l}45 \\
25 \\
30\end{array}$ & $\begin{array}{l}2836 \\
2380 \\
1118\end{array}$ & $\begin{array}{l}145 \\
150 \\
141 \\
173\end{array}$ & $\begin{array}{r}2316 \\
1863 \\
621\end{array}$ & $\begin{array}{l}26 \\
24 \\
31 \\
32\end{array}$ & $\begin{array}{r}2290 \\
1839 \\
590\end{array}$ & $\begin{array}{r}75 \\
77 \\
79 \\
84 \\
102\end{array}$ & $\begin{array}{r}2131 \\
1681 \\
438 \\
144 \\
309\end{array}$ & $\begin{array}{r}98 \\
98 \\
105 \\
108 \\
114\end{array}$ & $\begin{array}{r}2033 \\
1583 \\
333 \\
36 \\
195\end{array}$ & $\begin{array}{l}65 \\
65 \\
85 \\
40 \\
48\end{array}$ & $\begin{array}{r}1738 \\
1309 \\
60 \\
-200 \\
-49\end{array}$ & $\begin{array}{r}64 \\
113 \\
66 \\
86 \\
100\end{array}$ & $\begin{array}{r}1674 \\
1196 \\
-19 \\
-286 \\
-149\end{array}$ & $\begin{array}{l}65 \\
50 \\
70 \\
40 \\
68\end{array}$ & $\begin{array}{r}1463 \\
986 \\
-237 \\
-518 \\
-380\end{array}$ & $\begin{array}{r}115 \\
100 \\
53 \\
80 \\
88\end{array}$ & $\begin{array}{r}1301 \\
850 \\
-394 \\
-554\end{array}$ & $\begin{array}{l}75 \\
4 ! \\
73\end{array}$ & $\begin{array}{l}3035 \\
3565 \\
4470 \\
4506 \\
5424\end{array}$ & $\begin{array}{l}110 \\
120 \\
127 \\
112 \\
124\end{array}$ & 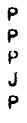 \\
\hline $\begin{array}{l}594 \\
130\end{array}$ & 10 & $\begin{array}{r}514 \\
50\end{array}$ & 0 & $\begin{array}{r}201 \\
-276\end{array}$ & 78 & $\begin{array}{l}-291 \\
-666 \\
-745\end{array}$ & $\begin{array}{l}29 \\
25\end{array}$ & $\begin{array}{l}-320 \\
-678 \\
-771\end{array}$ & $\begin{array}{r}98 \\
93 \\
109\end{array}$ & $\begin{array}{l}-489 \\
-851 \\
-945\end{array}$ & $\begin{array}{l}91 \\
80 \\
91\end{array}$ & $\begin{array}{r}-580 \\
-944 \\
-1036\end{array}$ & $\begin{array}{l}29 \\
20 \\
29\end{array}$ & $\begin{array}{r}-833 \\
-1208 \\
-1256\end{array}$ & $\begin{array}{r}130 \\
53 \\
49\end{array}$ & $\begin{array}{r}-963 \\
-1261 \\
-1305\end{array}$ & $\begin{array}{l}43 \\
20 \\
70\end{array}$ & $\begin{array}{l}-1103 \\
-1480 \\
-1560\end{array}$ & $\begin{array}{l}80 \\
70 \\
55\end{array}$ & -1284 & 75 & 5542 & 136 & $P$ \\
\hline 217 & 10 & 131 & 0 & -199 & 63 & -651 & 26 & -677 & 108 & -849 & 93 & -942 & 35 & -1179 & 57 & -1236 & 40 & -1414 & 70 & -1629 & 75 & 5600 & 145 & $p$ \\
\hline $\begin{array}{r}662 \\
1147\end{array}$ & $\begin{array}{l}10 \\
10\end{array}$ & $\begin{array}{r}567 \\
1047\end{array}$ & $\begin{array}{r}0 \\
10\end{array}$ & $\begin{array}{l}229 \\
677\end{array}$ & $\begin{array}{l}45 \\
54\end{array}$ & $\begin{array}{r}-223 \\
223 \\
1152 \\
862\end{array}$ & $\begin{array}{l}27 \\
16\end{array}$ & $\begin{array}{r}-250 \\
207 \\
1132 \\
845\end{array}$ & $\begin{array}{l}117 \\
118 \\
123 \\
137 \\
131\end{array}$ & $\begin{array}{r}-431 \\
30 \\
564 \\
933 \\
651\end{array}$ & $\begin{array}{l}97 \\
87 \\
85 \\
78 \\
79\end{array}$ & $\begin{array}{r}-528 \\
-57 \\
479 \\
855 \\
572\end{array}$ & $\begin{array}{l}34 \\
46 \\
80 \\
60 \\
73\end{array}$ & $\begin{array}{r}-743 \\
-327 \\
189 \\
652 \\
374\end{array}$ & $\begin{array}{l}45 \\
43 \\
55 \\
93 \\
35\end{array}$ & $\begin{array}{r}-788 \\
-370 \\
134 \\
559 \\
339\end{array}$ & $\begin{array}{l}68 \\
33 \\
30 \\
50 \\
43\end{array}$ & $\begin{array}{r}-1012 \\
-543 \\
-19 \\
452 \\
130\end{array}$ & $\begin{array}{l}60 \\
40 \\
40 \\
45 \\
66\end{array}$ & $\begin{array}{r}-1218 \\
-751 \\
352 \\
-78\end{array}$ & $\begin{array}{l}75 \\
90\end{array}$ & $\begin{array}{l}5110 \\
4439 \\
4249\end{array}$ & $\begin{array}{l}140 \\
130 \\
145\end{array}$ & $P$ \\
\hline $\begin{array}{r}-724 \\
-1177 \\
-1240\end{array}$ & $\begin{array}{r}5 \\
20 \\
30\end{array}$ & $\begin{array}{r}-774 \\
-1222 \\
-1273\end{array}$ & $\begin{array}{l}0 \\
0 \\
0\end{array}$ & $\begin{array}{l}-1222 \\
-1668 \\
-1739\end{array}$ & $\begin{array}{l}46 \\
36 \\
23\end{array}$ & $\begin{array}{l}-1598 \\
-1960 \\
-2038\end{array}$ & $\begin{array}{l}23 \\
42 \\
18\end{array}$ & $\begin{array}{l}-1621 \\
-2035 \\
-2082\end{array}$ & $\begin{array}{l}108 \\
125 \\
123 \\
107 \\
102\end{array}$ & $\begin{array}{r}-1430 \\
480 \\
-1819 \\
-2261 \\
-2310\end{array}$ & $\begin{array}{l}95 \\
80 \\
77 \\
80 \\
82\end{array}$ & $\begin{array}{r}-1527 \\
400 \\
-1896 \\
-2341 \\
-2392\end{array}$ & $\begin{array}{l}70 \\
40 \\
45 \\
46 \\
36\end{array}$ & $\begin{array}{r}-1740 \\
213 \\
-2051 \\
-2505 \\
-2586\end{array}$ & $\begin{array}{r}90 \\
140 \\
37 \\
75 \\
32\end{array}$ & $\begin{array}{r}-1830 \\
61 \\
-2088\end{array}$ & $\begin{array}{l}70 \\
25 \\
43\end{array}$ & $\begin{array}{r}-1968 \\
18 \\
-2291\end{array}$ & $\begin{array}{l}40 \\
60 \\
40\end{array}$ & -2524 & 140 & $\begin{array}{l}6739 \\
4331 \\
6694 \\
5774 \\
5634\end{array}$ & $\begin{array}{l}72 \\
103 \\
168 \\
140 \\
130\end{array}$ & J \\
\hline $\begin{array}{l}-1293 \\
-1296 \\
-1556 \\
-348\end{array}$ & $\begin{array}{l}20 \\
30 \\
70\end{array}$ & $\begin{array}{r}-1336 \\
-1321 \\
-1634 \\
-428\end{array}$ & $\begin{array}{l}0 \\
0 \\
0\end{array}$ & $\begin{array}{r}-1767 \\
-1778 \\
-1903 \\
-859\end{array}$ & $\begin{array}{l}34 \\
30 \\
20 \\
15\end{array}$ & $\begin{array}{l}-2055 \\
-2052 \\
-2036 \\
-2277 \\
-1086\end{array}$ & $\begin{array}{l}27 \\
35 \\
54 \\
50 \\
17\end{array}$ & $\begin{array}{l}-2103 \\
-2097 \\
-2105 \\
-2356 \\
-1139\end{array}$ & $\begin{array}{r}98 \\
100 \\
85 \\
110 \\
92\end{array}$ & $\begin{array}{l}-2334 \\
-2331 \\
-2338 \\
-2623 \\
-1436\end{array}$ & $\begin{array}{r}92 \\
75 \\
108 \\
85 \\
88\end{array}$ & $\begin{array}{l}-2426 \\
-2411 \\
-2446 \\
-2726 \\
-1524\end{array}$ & $\begin{array}{l}35 \\
80 \\
53 \\
50 \\
30\end{array}$ & $\begin{array}{l}-2616 \\
-2575 \\
-2621 \\
-2973\end{array}$ & $\begin{array}{l}21 \\
59 \\
24 \\
15\end{array}$ & $\begin{array}{l}-2634 \\
-2645 \\
-3000 \\
-1770\end{array}$ & $\begin{array}{l}0 \\
5 \\
0\end{array}$ & $\begin{array}{l}-2683 \\
-3050 \\
-1801\end{array}$ & $\begin{array}{l}30 \\
30 \\
45\end{array}$ & & $\begin{array}{r}95 \\
80 \\
255\end{array}$ & $\begin{array}{r}5437 \\
7534 \\
9100 \\
10260 \\
9203\end{array}$ & $\begin{array}{l}86 \\
168 \\
200 \\
196 \\
179\end{array}$ & $\begin{array}{l}1 \\
1 \\
1\end{array}$ \\
\hline-212 & 75 & -297 & 0 & 744 & 17 & $\begin{array}{r}-961 \\
-954 \\
-1788 \\
3454\end{array}$ & $\begin{array}{l}30 \\
28 \\
30 \\
12\end{array}$ & $\begin{array}{r}-1009 \\
-1016 \\
-1822 \\
3422\end{array}$ & $\begin{array}{l}75 \\
70 \\
60 \\
61\end{array}$ & $\begin{array}{r}-1291 \\
-1279 \\
-2127 \\
3252\end{array}$ & $\begin{array}{r}52 \\
105 \\
95 \\
95\end{array}$ & $\begin{array}{r}-1343 \\
-1384 \\
-2222 \\
4290 \\
3157\end{array}$ & $\begin{array}{l}65 \\
35 \\
30 \\
32 \\
28\end{array}$ & $\begin{array}{r}-1441 \\
-2378 \\
3980 \\
2864\end{array}$ & $\begin{array}{l}45 \\
11 \\
125 \\
100\end{array}$ & $\begin{array}{r}-1486 \\
-1634 \\
-2389 \\
3855 \\
2764\end{array}$ & $\begin{array}{r}0 \\
85 \\
80\end{array}$ & $\begin{array}{r}-1549 \\
-1659 \\
3564 \\
2557\end{array}$ & $\begin{array}{l}50 \\
70 \\
85\end{array}$ & $\begin{array}{r}-1872 \\
-2185 \\
3488 \\
2408\end{array}$ & $\begin{array}{r}325 \\
20 \\
75\end{array}$ & $\begin{array}{l}9109 \\
2260 \\
2680\end{array}$ & $\begin{array}{r}177 \\
115 \\
92\end{array}$ & r \\
\hline $\begin{array}{l}2773 \\
1978\end{array}$ & $\begin{array}{r}30 \\
0\end{array}$ & $\begin{array}{l}2721 \\
1923\end{array}$ & $\begin{array}{l}0 \\
0\end{array}$ & $\begin{array}{l}2440 \\
1636\end{array}$ & $\begin{array}{l}30 \\
30\end{array}$ & $\begin{array}{l}3797 \\
3409 \\
1915 \\
1123\end{array}$ & $\begin{array}{l}18 \\
20 \\
20\end{array}$ & $\begin{array}{l}3764 \\
3379 \\
1883 \\
1099\end{array}$ & $\begin{array}{l}60 \\
76 \\
72 \\
78 \\
80\end{array}$ & $\begin{array}{r}3590 \\
3189 \\
1723 \\
941 \\
1282\end{array}$ & $\begin{array}{r}90 \\
95 \\
75 \\
105 \\
109\end{array}$ & $\begin{array}{r}3500 \\
3072 \\
1618 \\
836 \\
1173\end{array}$ & $\begin{array}{l}95 \\
95 \\
55 \\
55 \\
50\end{array}$ & $\begin{array}{r}3228 \\
2724 \\
1358 \\
585 \\
934\end{array}$ & $\begin{array}{r}50 \\
25 \\
38 \\
101 \\
101\end{array}$ & $\begin{array}{r}3178 \\
2699 \\
1320 \\
484 \\
803\end{array}$ & $\begin{array}{r}75 \\
160 \\
130 \\
70 \\
30\end{array}$ & $\begin{array}{r}2232 \\
973 \\
231 \\
619\end{array}$ & $\begin{array}{l}65 \\
75 \\
80 \\
55 \\
25\end{array}$ & $\begin{array}{r}2807 \\
2142 \\
885 \\
128\end{array}$ & $\begin{array}{r}10 \\
110 \\
40 \\
40\end{array}$ & $\begin{array}{l}970 \\
408 \\
665 \\
939 \\
854\end{array}$ & $\begin{array}{r}78 \\
121 \\
96 \\
118 \\
144\end{array}$ & $\begin{array}{l}P \\
P \\
J\end{array}$ \\
\hline $\begin{array}{r}176 \\
144 \\
92\end{array}$ & $\begin{array}{l}10 \\
20 \\
15\end{array}$ & 85 & $\begin{array}{l}0 \\
5\end{array}$ & 537 & $\begin{array}{r}65 \\
75\end{array}$ & $\begin{array}{r}914 \\
594 \\
61\end{array}$ & & $\begin{array}{r}890 \\
575 \\
36\end{array}$ & $\begin{array}{r}84 \\
79 \\
84 \\
93 \\
107\end{array}$ & $\begin{array}{r}781 \\
1172 \\
722 \\
407 \\
-134\end{array}$ & $\begin{array}{r}104 \\
85 \\
110 \\
117 \\
92\end{array}$ & $\begin{array}{r}677 \\
1058 \\
612 \\
290 \\
-226\end{array}$ & 10 & $\begin{array}{r}430 \\
827 \\
380 \\
82 \\
-457\end{array}$ & $\begin{array}{r}74 \\
91 \\
90 \\
101 \\
37\end{array}$ & $\begin{array}{r}356 \\
736 \\
290 \\
-19 \\
-494\end{array}$ & $\begin{array}{l}60 \\
45 \\
60 \\
65 \\
45\end{array}$ & $\begin{array}{r}62 \\
512 \\
39 \\
-260 \\
-757\end{array}$ & $\begin{array}{l}30 \\
30 \\
45 \\
30 \\
40\end{array}$ & $\begin{array}{r}-36 \\
-78 \\
-393 \\
-903\end{array}$ & $\begin{array}{r}80 \\
125 \\
95\end{array}$ & $\begin{array}{l}3946 \\
3620 \\
4107 \\
4103 \\
4663\end{array}$ & $\begin{array}{l}107 \\
119 \\
134\end{array}$ & $\begin{array}{l}P \\
J\end{array}$ \\
\hline $\begin{array}{r}1096 \\
686 \\
98 \\
269 \\
797\end{array}$ & $\begin{array}{l}20 \\
10 \\
15 \\
20\end{array}$ & $\begin{array}{r}605 \\
9 \\
177 \\
700\end{array}$ & 0 & $\begin{array}{r}716 \\
272 \\
-349 \\
-171 \\
339\end{array}$ & & $\begin{array}{l}-811 \\
-583 \\
-111\end{array}$ & 2 & $\begin{array}{l}-211 \\
-834 \\
-605 \\
-133\end{array}$ & $\begin{array}{l}106 \\
105 \\
120 \\
113 \\
126\end{array}$ & $\begin{array}{r}54 \\
-382 \\
-1019 \\
-785 \\
-321\end{array}$ & $\begin{array}{l}99 \\
97 \\
90 \\
99 \\
82\end{array}$ & $\begin{array}{r}-45 \\
-479 \\
-1109 \\
-884 \\
-403\end{array}$ & $\begin{array}{l}45 \\
25 \\
20 \\
40 \\
57\end{array}$ & $\begin{array}{r}-275 \\
-634 \\
-1307 \\
-1081 \\
-634\end{array}$ & $\begin{array}{l}34 \\
87 \\
92 \\
77 \\
69\end{array}$ & $\begin{array}{r}-30 \\
-73 \\
-139 \\
-115 \\
-70\end{array}$ & $\begin{array}{l}31 \\
35 \\
38 \\
38 \\
50\end{array}$ & $\begin{array}{r}-949 \\
-1567 \\
-1353 \\
-881\end{array}$ & $\begin{array}{l}50 \\
30 \\
53 \\
65 \\
75\end{array}$ & & $\begin{array}{l}20 \\
135 \\
125 \\
130 \\
120\end{array}$ & $\begin{array}{l}743 \\
144 \\
457 \\
569 \\
067\end{array}$ & $\begin{array}{l}150 \\
140 \\
159 \\
131 \\
143\end{array}$ & $\begin{array}{l}p \\
p \\
p\end{array}$ \\
\hline 2631 & 15 & -1158 & 0 & -1545 & $\begin{array}{l}60 \\
41 \\
45\end{array}$ & $\begin{array}{r}1670 \\
1698 \\
-1688 \\
-1913\end{array}$ & $\begin{array}{l}16 \\
10 \\
10 \\
10\end{array}$ & $\begin{array}{r}1654 \\
1688 \\
-1718 \\
-1923\end{array}$ & $\begin{array}{l}125 \\
130 \\
135 \\
122 \\
110\end{array}$ & $\begin{array}{r}335 \\
1459 \\
1480 \\
-1962 \\
-2131\end{array}$ & $\begin{array}{l}80 \\
88 \\
73 \\
75 \\
72\end{array}$ & $\begin{array}{r}24 \\
137 \\
140 \\
-203\end{array}$ & $\begin{array}{l}0 \\
0 \\
3\end{array}$ & $\begin{array}{r}1 \\
118 \\
127 \\
-227\end{array}$ & $\begin{array}{l}42 \\
73 \\
67 \\
40\end{array}$ & $\begin{array}{r}-2 \\
111 \\
121 \\
-231\end{array}$ & $\begin{array}{l}20 \\
95 \\
44\end{array}$ & $\begin{array}{r}-209 \\
927 \\
948\end{array}$ & $\begin{array}{l}35 \\
60\end{array}$ & 745 & $\begin{array}{l}175 \\
45\end{array}$ & $\begin{array}{l}4155 \\
2994 \\
4022 \\
5136\end{array}$ & $\begin{array}{l}117 \\
106 \\
140 \\
128\end{array}$ & k \\
\hline $\begin{array}{l}012 \\
077 \\
186\end{array}$ & $\begin{array}{l}10 \\
10 \\
25 \\
30 \\
30\end{array}$ & $\begin{array}{l}-109 \\
-115 \\
-126 \\
-116 \\
-128\end{array}$ & $\begin{array}{l}0 \\
0 \\
0 \\
0 \\
0\end{array}$ & $\begin{array}{l}-15 \\
-15 \\
-16 \\
-15 \\
-17\end{array}$ & $\begin{array}{l}46 \\
45 \\
35 \\
34 \\
31\end{array}$ & $\begin{array}{l}-2000 \\
-189 \\
-202\end{array}$ & $\begin{array}{l}25 \\
17 \\
28 \\
28 \\
32\end{array}$ & $\begin{array}{l}-1 \\
-1 \\
-2 \\
-1 \\
-2\end{array}$ & $\begin{array}{r}113 \\
117 \\
108 \\
102 \\
85\end{array}$ & $\begin{array}{l}-2 \\
-2 \\
-2 \\
-2 \\
-2\end{array}$ & $\begin{array}{l}84 \\
81 \\
75 \\
85 \\
98\end{array}$ & $\begin{array}{l}-2187 \\
-2275 \\
-2350 \\
-2252 \\
-2402\end{array}$ & $\begin{array}{l}34 \\
22 \\
20 \\
40 \\
75\end{array}$ & $\begin{array}{l}-233 \\
-245 \\
-251 \\
-242 \\
-259\end{array}$ & $\begin{array}{l}62 \\
70\end{array}$ & -2401 & 25 & -2524 & 40 & -2796 & 105 & & $\begin{array}{l}162 \\
110 \\
120 \\
140 \\
108\end{array}$ & $\mathrm{~J}$ \\
\hline $\begin{array}{l}-1372 \\
-1503 \\
-1595 \\
-1627 \\
-228\end{array}$ & $\begin{array}{l}35 \\
35 \\
35 \\
50 \\
81\end{array}$ & $\begin{array}{l}-1428 \\
-1540 \\
-1646 \\
-1688 \\
-309\end{array}$ & $\begin{array}{l}0 \\
0 \\
0 \\
0 \\
0\end{array}$ & $\begin{array}{l}-1853 \\
-1989 \\
-2079 \\
-2140 \\
-754\end{array}$ & $\begin{array}{l}31 \\
20 \\
12 \\
20 \\
12\end{array}$ & $\begin{array}{l}-2096 \\
-2228 \\
-2295 \\
-2338 \\
-976\end{array}$ & $\begin{array}{l}28 \\
50 \\
60 \\
70 \\
27\end{array}$ & $\begin{array}{l}-21 \\
-22 \\
-23 \\
-24 \\
-10\end{array}$ & $\begin{array}{l}77 \\
78 \\
72 \\
75 \\
70\end{array}$ & $\begin{array}{l}-2397 \\
-2543 \\
-2615 \\
-2675 \\
-1307\end{array}$ & $\begin{array}{r}115 \\
106 \\
93 \\
91 \\
103\end{array}$ & $\begin{array}{l}-2512 \\
-2649 \\
-2708 \\
-2766 \\
-1410\end{array}$ & $\begin{array}{l}36 \\
46 \\
55 \\
95 \\
45\end{array}$ & $\begin{array}{l}-26 \\
-26 \\
-28 \\
-20 \\
-16\end{array}$ & $\begin{array}{l}63 \\
39 \\
50 \\
23 \\
10\end{array}$ & $\begin{array}{l}-286 \\
-294 \\
-299 \\
-167\end{array}$ & $\begin{array}{l}0 \\
0 \\
0 \\
0\end{array}$ & & 5 & & 0 & $\begin{array}{l}5153 \\
5486 \\
5540 \\
5724 \\
9040\end{array}$ & $\begin{array}{l}126 \\
130 \\
126 \\
134 \\
179\end{array}$ & $\begin{array}{l}\mathrm{J} \\
\mathrm{J} \\
\mathrm{J} \\
\mathrm{J} \\
\mathrm{J}\end{array}$ \\
\hline $\begin{array}{r}-251 \\
-647 \\
-880 \\
1099 \\
234\end{array}$ & $\begin{array}{l}70 \\
80 \\
85 \\
30 \\
70\end{array}$ & $\begin{array}{r}-341 \\
-757 \\
-1000 \\
1000 \\
126\end{array}$ & $\begin{array}{r}0 \\
0 \\
0 \\
10 \\
0\end{array}$ & $\begin{array}{r}-818 \\
-1219 \\
-1453 \\
879 \\
-48\end{array}$ & $\begin{array}{r}13 \\
9 \\
110 \\
55\end{array}$ & $\begin{array}{r}-101 \\
-140 \\
-163 \\
360 \\
-51\end{array}$ & $\begin{array}{l}27 \\
45 \\
30 \\
40 \\
25\end{array}$ & $\begin{array}{r}-1081 \\
-1474 \\
-1715 \\
324 \\
-572\end{array}$ & $\begin{array}{l}75 \\
47 \\
85 \\
52 \\
50\end{array}$ & $\begin{array}{r}-1385 \\
-1690 \\
-1985 \\
12 \\
-787\end{array}$ & $\begin{array}{l}48 \\
68 \\
80 \\
73 \\
70\end{array}$ & $\begin{array}{r}-1433 \\
-1764 \\
-2065 \\
-63 \\
-874\end{array}$ & $\begin{array}{l}60 \\
50 \\
55 \\
85 \\
85\end{array}$ & $\begin{array}{r}-1994 \\
-2315 \\
-416 \\
-1185\end{array}$ & $\begin{array}{l}20 \\
30 \\
35 \\
50 \\
65\end{array}$ & $\begin{array}{r}-2024 \\
-2350 \\
-468 \\
-1251\end{array}$ & $\begin{array}{r}0 \\
0 \\
0 \\
65 \\
80\end{array}$ & $\begin{array}{r}-2049 \\
-2375 \\
-692 \\
-1523\end{array}$ & $\begin{array}{r}15 \\
0 \\
0 \\
70 \\
10\end{array}$ & $\begin{array}{l}-2616 \\
-2815 \\
-1635\end{array}$ & $\begin{array}{l}285 \\
210 \\
280\end{array}$ & $\begin{array}{r}9122 \\
10333 \\
10708 \\
7484 \\
7308\end{array}$ & $\begin{array}{l}173 \\
197 \\
212\end{array}$ & $\begin{array}{l}T \\
p \\
p\end{array}$ \\
\hline
\end{tabular}


TABLE 2. - SELECTED GEOLOGIC DATA -- CONTINUED

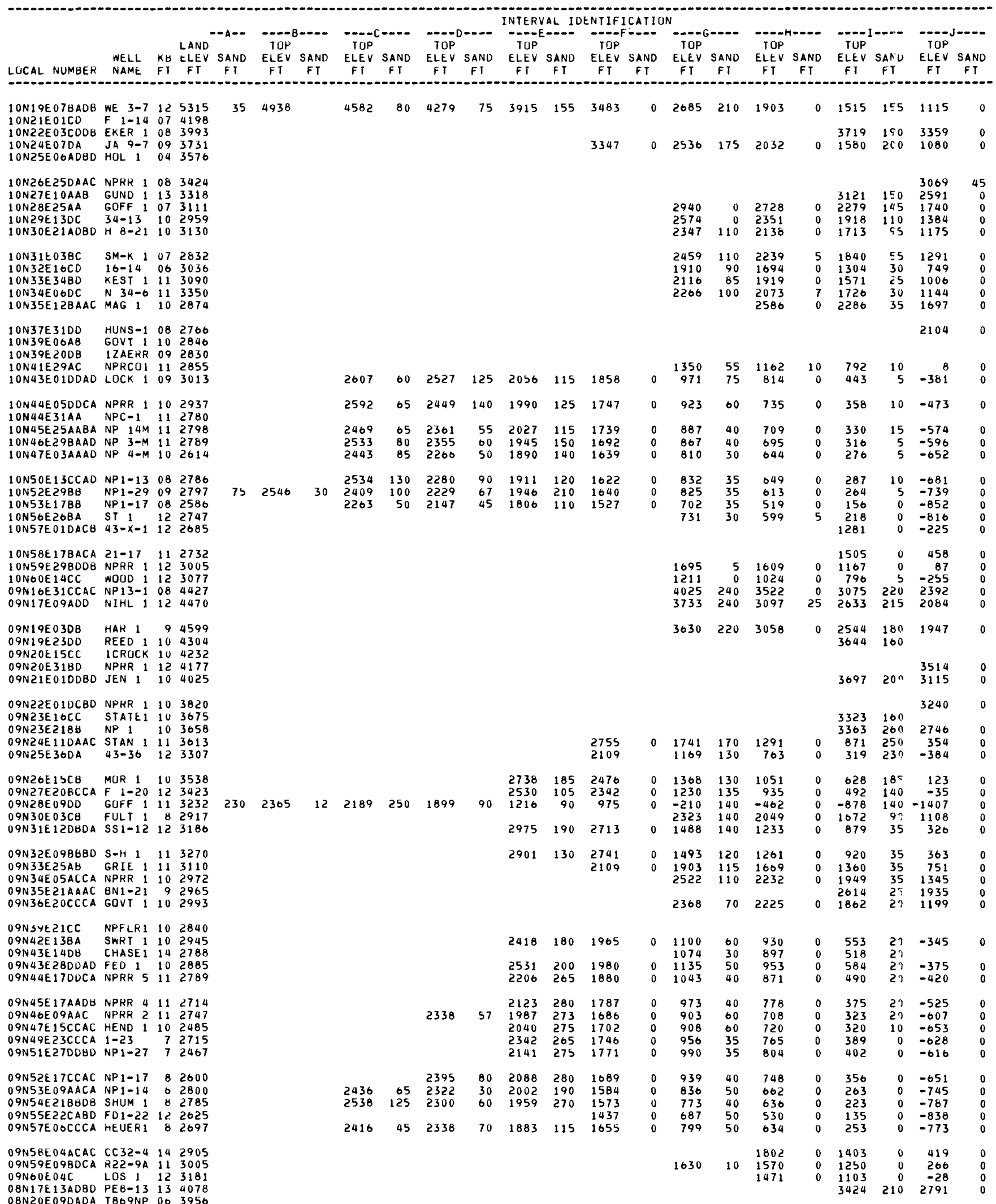




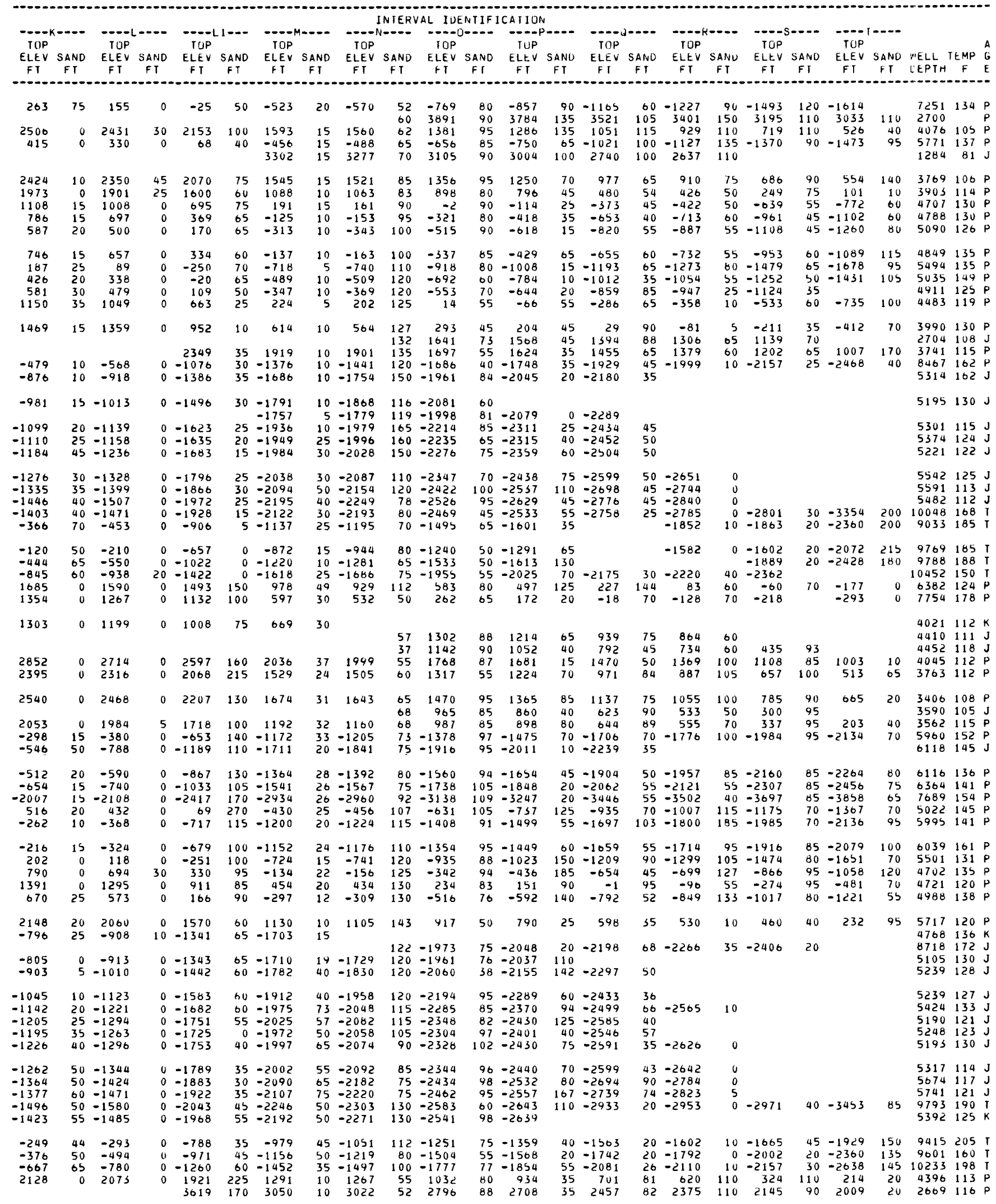




\begin{tabular}{|c|c|c|c|c|c|c|c|c|c|c|c|c|c|c|c|c|c|c|c|c|c|c|}
\hline LOCAL NUMBER & $\begin{array}{l}\text { WELL } \\
\text { NAME }\end{array}$ & $\begin{array}{l}K B \\
F T\end{array}$ & $\begin{array}{l}\text { LAND } \\
\text { ELEV } \\
\text { FI }\end{array}$ & $\begin{array}{l}\text { SAND } \\
\text { FT } \\
\text { FT }\end{array}$ & $\begin{array}{l}\text { TOP } \\
\text { ELEV } \\
\text { FT }\end{array}$ & SAND & $\begin{array}{l}\text { TOP } \\
\text { ELEV } \\
\text { FI }\end{array}$ & $\begin{array}{l}\text { SAND } \\
\text { FT }\end{array}$ & $\begin{array}{l}O A O D \\
\text { ELEV } \\
\text { FI }\end{array}$ & $\begin{array}{l}\text { SAND } \\
\text { FI }\end{array}$ & \begin{tabular}{c} 
INTER \\
\hdashline TOP \\
ELEV \\
FT
\end{tabular} & $\begin{array}{l}\text { VAL ID } \\
\text { E...- } \\
\text { SAND } \\
\text { FT }\end{array}$ & \begin{tabular}{c} 
ENTIF \\
\hdashline$T O P$ \\
ELEV \\
FT
\end{tabular} & $\begin{array}{l}\text { ICATIC } \\
\text { FA- } \\
\text { SAND } \\
\text { FI }\end{array}$ & $\begin{array}{c}\text { FI } \\
\text { TOP } \\
\text { ELEV }\end{array}$ & $\begin{array}{c}\text { SAND } \\
\text { FI }\end{array}$ & $\begin{array}{l}O Z-H \\
\text { TOP } \\
\text { FLT }\end{array}$ & $\begin{array}{l}\text { SAND } \\
\text { FT }\end{array}$ & $\begin{array}{c}\text { YOP } \\
\text { ELEV } \\
\text { FT }\end{array}$ & $\begin{array}{c}\text { SANU } \\
\text { FT }\end{array}$ & $\begin{array}{l}\text { TOP } \\
\text { ELEV } \\
F T\end{array}$ & $\begin{array}{c}\text { SAND } \\
\text { FT }\end{array}$ \\
\hline $\begin{array}{l}\text { OBN24E 02BBBB } \\
\text { OBN25E O8AAA } \\
\text { OBN26E 06AADC } \\
\text { OBN27E22AA } \\
\text { OBN28E3ODC }\end{array}$ & $\begin{array}{l}\text { GHALL1 } \\
\text { SSPUR I } \\
\text { LIND-1 } \\
\text { IGOFFE } \\
\text { BN-1 }\end{array}$ & $\begin{array}{r}12 \\
08 \\
08 \\
8 \\
08\end{array}$ & $\begin{array}{l}3628 \\
3549 \\
3240 \\
3402 \\
3336\end{array}$ & 320 & 2994 & 0 & 2834 & 140 & 2561 & 120 & $\begin{array}{l}2750 \\
2422 \\
1949\end{array}$ & $\begin{array}{r}125 \\
85 \\
\\
115\end{array}$ & $\begin{array}{l}2527 \\
2108 \\
1666 \\
1664\end{array}$ & $\begin{array}{l}0 \\
0 \\
0 \\
0\end{array}$ & $\begin{array}{r}2724 \\
1540 \\
1168 \\
640 \\
658\end{array}$ & $\begin{array}{l}210 \\
220 \\
155 \\
110 \\
110\end{array}$ & $\begin{array}{r}2320 \\
1132 \\
748 \\
370 \\
344\end{array}$ & $\begin{array}{l}0 \\
0 \\
0 \\
0 \\
0\end{array}$ & $\begin{array}{r}1904 \\
687 \\
337 \\
-79 \\
-74\end{array}$ & $\begin{array}{l}220 \\
220 \\
230 \\
140 \\
130\end{array}$ & $\begin{array}{r}1290 \\
98 \\
-193 \\
-644\end{array}$ & $\begin{array}{l}0 \\
0 \\
0 \\
0\end{array}$ \\
\hline $\begin{array}{l}\text { OBN29E25CADB } \\
\text { OBN3OE 14DD } \\
\text { OBN31E29BCBD } \\
\text { OBN32E 03BCAB } \\
\text { OBN33E 03AAA }\end{array}$ & $\begin{array}{l}1-25 N P \\
\text { HLND-1 } \\
\text { NP } Y-1 \\
\text { TREAS 1 } \\
\text { LUSK-1 }\end{array}$ & $\begin{array}{l}12 \\
12 \\
08 \\
08 \\
06\end{array}$ & $\begin{array}{l}3328 \\
3378 \\
3525 \\
3264 \\
3133\end{array}$ & $\begin{array}{l}470 \\
455 \\
560\end{array}$ & $\begin{array}{l}2810 \\
2800 \\
2959\end{array}$ & $\begin{array}{l}0 \\
0 \\
0\end{array}$ & $\begin{array}{l}2702 \\
2660 \\
2779\end{array}$ & $\begin{array}{l}125 \\
190 \\
230\end{array}$ & $\begin{array}{l}2400 \\
2357 \\
2501 \\
2912\end{array}$ & $\begin{array}{l}145 \\
145 \\
205 \\
215\end{array}$ & $\begin{array}{l}1755 \\
1743 \\
1930 \\
2411 \\
2796\end{array}$ & $\begin{array}{r}65 \\
115 \\
120 \\
200 \\
80\end{array}$ & $\begin{array}{l}1616 \\
1543 \\
1685 \\
2072 \\
2642\end{array}$ & $\begin{array}{l}0 \\
0 \\
0 \\
0 \\
0\end{array}$ & $\begin{array}{r}642 \\
430 \\
570 \\
966 \\
1509\end{array}$ & $\begin{array}{r}100 \\
90 \\
95 \\
100 \\
100\end{array}$ & $\begin{array}{r}289 \\
238 \\
370 \\
745 \\
1277\end{array}$ & $\begin{array}{l}0 \\
0 \\
0 \\
0 \\
0\end{array}$ & $\begin{array}{r}-113 \\
-167 \\
-32 \\
357 \\
971\end{array}$ & $\begin{array}{r}110 \\
120 \\
75 \\
45 \\
45\end{array}$ & $\begin{array}{r}-703 \\
-719 \\
-537 \\
-181 \\
397\end{array}$ & $\begin{array}{l}0 \\
0 \\
0 \\
0 \\
0\end{array}$ \\
\hline $\begin{array}{l}\text { OBN35E 29AC } \\
\text { OBN36E19CC } \\
\text { OBN36E 21DB } \\
\text { 08N37E 05CD } \\
\text { O8N39E26BC }\end{array}$ & $\begin{array}{l}\text { NP } 1 \\
N P 1-19 \\
N 2-149 \\
1 \text { MAGE } \\
1 \text { GOVT }\end{array}$ & $\begin{array}{l}8 \\
10 \\
10 \\
10 \\
10\end{array}$ & $\begin{array}{l}2898 \\
2812 \\
2767 \\
2918 \\
2790\end{array}$ & & & & & & & & & & & & $\begin{array}{l}2358 \\
2592 \\
2670\end{array}$ & $\begin{array}{r}100 \\
70 \\
50\end{array}$ & $\begin{array}{l}2076 \\
2317 \\
2563\end{array}$ & $\begin{array}{r}0 \\
0 \\
20\end{array}$ & $\begin{array}{l}1778 \\
2031 \\
2210 \\
2173\end{array}$ & $\begin{array}{l}30 \\
30 \\
30 \\
20\end{array}$ & 1562 & 0 \\
\hline $\begin{array}{l}\text { OAN } 40 E 09 A A \\
\text { OBN40E14CD } \\
\text { OBN41E23DCDB } \\
\text { OBN42E 11AAC } \\
\text { OBN43E OZAB }\end{array}$ & $\begin{array}{l}\text { NPRR - 1 } \\
\text { LEE 1 } \\
B N-3 \\
B N-1 \\
\text { USA } 1\end{array}$ & $\begin{array}{l}07 \\
12 \\
10 \\
10 \\
11\end{array}$ & $\begin{array}{l}2676 \\
2709 \\
2848 \\
2899 \\
3016\end{array}$ & & & & & & $\begin{array}{l}2604 \\
2797\end{array}$ & $\begin{array}{l}150 \\
120\end{array}$ & $\begin{array}{l}2318 \\
2299 \\
2427\end{array}$ & $\begin{array}{l}200 \\
200 \\
305\end{array}$ & $\begin{array}{l}2084 \\
1995 \\
1957\end{array}$ & $\begin{array}{l}0 \\
0 \\
0\end{array}$ & $\begin{array}{l}1195 \\
1141 \\
1144\end{array}$ & $\begin{array}{l}60 \\
70 \\
70\end{array}$ & $\begin{array}{r}1051 \\
982 \\
952\end{array}$ & $\begin{array}{l}0 \\
0 \\
0\end{array}$ & $\begin{array}{l}624 \\
597 \\
587\end{array}$ & $\begin{array}{l}25 \\
20 \\
10\end{array}$ & $\begin{array}{l}-176 \\
-232 \\
-381\end{array}$ & $\begin{array}{l}0 \\
0 \\
0\end{array}$ \\
\hline 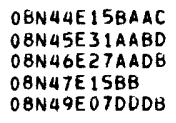 & $\begin{array}{l}\text { SNYDE } 8 \\
\text { NPRR 9 } \\
\text { NPI } 1-27 \\
\text { SNYDE 1 } \\
\text { NPRI }=7\end{array}$ & $\begin{array}{l}11 \\
11 \\
10 \\
12 \\
08\end{array}$ & $\begin{array}{l}2794 \\
2609 \\
2634 \\
2616 \\
2665\end{array}$ & & & & & & & & $\begin{array}{l}2179 \\
2177 \\
2180 \\
2075 \\
2101\end{array}$ & $\begin{array}{l}215 \\
240 \\
285 \\
185 \\
250\end{array}$ & $\begin{array}{l}1891 \\
1839 \\
1743 \\
1818 \\
1771\end{array}$ & $\begin{array}{l}0 \\
0 \\
0 \\
0 \\
0\end{array}$ & $\begin{array}{r}1046 \\
1003 \\
968 \\
1032 \\
995\end{array}$ & $\begin{array}{l}50 \\
45 \\
60 \\
50 \\
65\end{array}$ & $\begin{array}{l}871 \\
827 \\
784 \\
845 \\
824\end{array}$ & $\begin{array}{l}0 \\
0 \\
0 \\
0 \\
0\end{array}$ & $\begin{array}{l}503 \\
454 \\
394 \\
448 \\
397\end{array}$ & $\begin{array}{l}10 \\
15 \\
15 \\
15 \\
10\end{array}$ & $\begin{array}{l}-457 \\
-525 \\
-595 \\
-550 \\
-607\end{array}$ & $\begin{array}{l}0 \\
0 \\
0 \\
0 \\
0\end{array}$ \\
\hline $\begin{array}{l}\text { OBN50E } 1 \text { 1 DDBD } \\
\text { OBN52E 19DDBD } \\
\text { OBN5 EE } 19 D D C \\
\text { OBN56E } 36 D D B B \\
\text { OBN57E } 1 \text { 2DUAE }\end{array}$ & $\begin{array}{l}\text { NP } 1-13 \\
\text { NP } 1=19 \\
S-19-1 \\
44-36 \\
\text { BUERG } 1\end{array}$ & $\begin{array}{r}08 \\
07 \\
8 \\
5 \\
12\end{array}$ & $\begin{array}{l}2930 \\
2751 \\
3063 \\
2756 \\
2889\end{array}$ & & & & 2514 & 125 & $\begin{array}{l}2534 \\
2301\end{array}$ & $\begin{array}{l}70 \\
35\end{array}$ & $\begin{array}{l}2214 \\
2062 \\
1961\end{array}$ & $\begin{array}{l}360 \\
205 \\
140\end{array}$ & $\begin{array}{l}1765 \\
1800 \\
1656 \\
1791\end{array}$ & $\begin{array}{l}0 \\
0 \\
0 \\
0\end{array}$ & $\begin{array}{r}1004 \\
1054 \\
830 \\
1129\end{array}$ & $\begin{array}{l}35 \\
55 \\
50 \\
30\end{array}$ & $\begin{array}{l}768 \\
865 \\
688 \\
945\end{array}$ & $\begin{array}{l}0 \\
0 \\
0 \\
0\end{array}$ & $\begin{array}{l}428 \\
466\end{array}$ & $\begin{array}{l}5 \\
0\end{array}$ & $\begin{array}{l}-582 \\
-561 \\
-511\end{array}$ & $\begin{array}{l}0 \\
0\end{array}$ \\
\hline $\begin{array}{l}\text { OBN59E } 26 C D A C \\
\text { OBN60E2 GCD } \\
\text { OBN6OE31AD } \\
\text { OBNGOE31CC } \\
\text { OBNGIE I9CCA }\end{array}$ & $\begin{array}{l}24-26 \\
F 24-26 \\
24 A-31 \\
F 13-31 \\
N P=1\end{array}$ & $\begin{array}{l}12 \\
12 \\
12 \\
11\end{array}$ & $\begin{array}{l}3032 \\
3125 \\
3014 \\
2988 \\
3124\end{array}$ & & & & & & & & & & 2882 & 0 & 2204 & 10 & 2094 & 0 & $\begin{array}{l}1646 \\
1662\end{array}$ & $\begin{array}{l}0 \\
0\end{array}$ & $\begin{array}{l}564 \\
606\end{array}$ & 0 \\
\hline $\begin{array}{l}\text { 07N11E13CCDB } \\
\text { O7N13E28DA } \\
\text { O7N16E18BACD } \\
\text { O7N19E09AC } \\
\text { O7N22E27CACA }\end{array}$ & $\begin{array}{l}\text { PU1-13 } \\
\text { FED } 1 \\
\text { AN3-18 } \\
\text { SHA A1 } \\
\text { JEN AI }\end{array}$ & $\begin{array}{l}09 \\
08 \\
06 \\
11 \\
11\end{array}$ & $\begin{array}{l}5830 \\
5156 \\
4303 \\
3962 \\
3568\end{array}$ & & & & & & & & & & & & & & & & & & 2869 & 0 \\
\hline 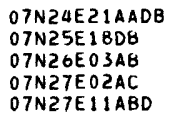 & $\begin{array}{l}\text { MAI }-21 \\
\text { IRATHS } \\
\text { BN3726 } \\
1-2 B U L \\
\text { WNITBI }\end{array}$ & $\begin{array}{l}06 \\
11 \\
12 \\
12\end{array}$ & $\begin{array}{l}3384 \\
3305 \\
3607 \\
3905 \\
3946\end{array}$ & & & $\cdot$ & & & & & $\begin{array}{l}2378 \\
2042\end{array}$ & 140 & $\begin{array}{l}2210 \\
1979 \\
1024 \\
1696 \\
1706\end{array}$ & $\begin{array}{l}0 \\
0 \\
0 \\
0 \\
0\end{array}$ & $\begin{array}{r}1330 \\
1150 \\
-49 \\
694 \\
716\end{array}$ & $\begin{array}{r}210 \\
85 \\
110 \\
115 \\
110\end{array}$ & $\begin{array}{r}880 \\
700 \\
-286 \\
432 \\
461\end{array}$ & $\begin{array}{l}0 \\
0 \\
0 \\
0 \\
0\end{array}$ & $\begin{array}{r}538 \\
366 \\
-775 \\
15 \\
31\end{array}$ & $\begin{array}{l}180 \\
185 \\
180 \\
140 \\
130\end{array}$ & $\begin{array}{r}-69 \\
-1361 \\
-568 \\
-224\end{array}$ & 0 \\
\hline $\begin{array}{l}\text { 07N2BE09AC } \\
07 N 2 B E 31 B A \\
07 N 29 E 17 A B C A \\
07 N 30 E 01 B A \\
07 N 30 E 21 A C\end{array}$ & $\begin{array}{l}\text { CHAN } 1 \\
\text { 1BN317 } \\
\text { NPRR } 1 \\
1 \text { GOVT } \\
1 \text { GOVT }\end{array}$ & $\begin{array}{r}11 \\
12 \\
10 \\
12 \\
9\end{array}$ & $\begin{array}{l}3450 \\
3912 \\
3821 \\
3534 \\
3465\end{array}$ & $\begin{array}{l}490 \\
590\end{array}$ & $\begin{array}{l}2903 \\
2976\end{array}$ & $\begin{array}{l}0 \\
0\end{array}$ & $\begin{array}{l}2793 \\
2852\end{array}$ & $\begin{array}{l}123 \\
103\end{array}$ & $\begin{array}{l}2660 \\
2739\end{array}$ & $\begin{array}{l}250 \\
240\end{array}$ & $\begin{array}{l}1952 \\
1905\end{array}$ & $\begin{array}{l}190 \\
125\end{array}$ & $\begin{array}{l}1615 \\
1647 \\
1711 \\
1597\end{array}$ & $\begin{array}{l}0 \\
0 \\
0 \\
0\end{array}$ & $\begin{array}{l}630 \\
731 \\
617 \\
539\end{array}$ & $\begin{array}{l}95 \\
85 \\
90 \\
90\end{array}$ & $\begin{array}{l}300 \\
384 \\
384 \\
309\end{array}$ & $\begin{array}{l}0 \\
0 \\
0 \\
0\end{array}$ & $\begin{array}{r}-105 \\
-2 \\
21 \\
55\end{array}$ & $\begin{array}{l}120 \\
120 \\
110 \\
120\end{array}$ & $\begin{array}{l}-687 \\
-593\end{array}$ & $\begin{array}{l}0 \\
0\end{array}$ \\
\hline $\begin{array}{l}\text { O7N31E04ACDH } \\
07 N 32 E 03 C C D B \\
07 N 32 E 18 A D \\
07 N 35 E 12 B D B D \\
07 N 38 E 03 C C\end{array}$ & $\begin{array}{l}\text { MAART } 1 \\
\text { JENNAI } \\
2 \\
\text { IREAS } 1 \\
\text { IERIKS }\end{array}$ & $\begin{array}{r}09 \\
10 \\
11 \\
9\end{array}$ & $\begin{array}{l}3416 \\
3122 \\
3453 \\
2848 \\
2780\end{array}$ & & & & & & & & $\begin{array}{l}2285 \\
2412\end{array}$ & $\begin{array}{l}220 \\
270\end{array}$ & $\begin{array}{l}2000 \\
2069 \\
2308\end{array}$ & $\begin{array}{l}0 \\
0\end{array}$ & $\begin{array}{r}945 \\
962 \\
1275 \\
2339\end{array}$ & $\begin{array}{l}95 \\
75 \\
100 \\
100\end{array}$ & $\begin{array}{r}701 \\
732 \\
1022 \\
2066\end{array}$ & $\begin{array}{l}0 \\
0 \\
0 \\
0\end{array}$ & $\begin{array}{r}360 \\
370 \\
677 \\
1783\end{array}$ & $\begin{array}{l}50 \\
30 \\
30\end{array}$ & $\begin{array}{l}-212 \\
-203 \\
1161\end{array}$ & $\begin{array}{l}0 \\
0\end{array}$ \\
\hline $\begin{array}{l}\text { OTN39E } 32 B D A C \\
\text { OTN4OEOIADB } \\
\text { OTN4IEIIAAC } \\
\text { OTN42E IGBD } \\
\text { OTN44EOTCB }\end{array}$ & $\begin{array}{l}\text { MON F1 } \\
3 \\
B N 2 \\
\text { MONI } 1 \\
\text { NP H-1 }\end{array}$ & $\begin{array}{l}11 \\
5 \\
10 \\
12 \\
10\end{array}$ & $\begin{array}{l}2802 \\
2765 \\
2760 \\
2847 \\
2702\end{array}$ & & & & & & & & $\begin{array}{l}2233 \\
2162\end{array}$ & $\begin{array}{l}170 \\
205\end{array}$ & $\begin{array}{l}2045 \\
1944\end{array}$ & $\begin{array}{l}0 \\
0\end{array}$ & $\begin{array}{l}1932 \\
1160 \\
1082\end{array}$ & $\begin{array}{l}50 \\
70 \\
60\end{array}$ & $\begin{array}{r}1804 \\
1012 \\
929\end{array}$ & $\begin{array}{l}0 \\
0\end{array}$ & $\begin{array}{r}2250 \\
585 \\
519\end{array}$ & $\begin{array}{r}0 \\
25 \\
20\end{array}$ & $\begin{array}{l}1398 \\
-237 \\
-327\end{array}$ & $\begin{array}{l}0 \\
0\end{array}$ \\
\hline $\begin{array}{l}\text { 07N47E32CA } \\
\text { O7N4BE21BEAA } \\
\text { O7N49EI ICDBD } \\
\text { O7N5OEO1BB } \\
\text { O7N5OE2ZDBAC }\end{array}$ & $\begin{array}{l}\text { GOVT } 1 \\
\text { BLUM } 1 \\
\text { NPI-30 } \\
1 \text { BIXLE } \\
\text { MCCAMI }\end{array}$ & $\begin{array}{r}10 \\
11 \\
05 \\
7 \\
10\end{array}$ & $\begin{array}{l}2660 \\
2702 \\
2759 \\
2989 \\
3107\end{array}$ & & & & 2684 & 70 & $\begin{array}{l}2447 \\
2607\end{array}$ & $\begin{array}{l}225 \\
115\end{array}$ & $\begin{array}{l}1981 \\
1912 \\
2122\end{array}$ & $\begin{array}{r}110 \\
95 \\
245\end{array}$ & $\begin{array}{l}1748 \\
1770 \\
1827\end{array}$ & $\begin{array}{l}0 \\
0 \\
0\end{array}$ & $\begin{array}{l}1008 \\
1004 \\
1062\end{array}$ & $\begin{array}{l}50 \\
50 \\
40\end{array}$ & $\begin{array}{l}839 \\
854 \\
880\end{array}$ & $\begin{array}{l}0 \\
0 \\
0\end{array}$ & $\begin{array}{l}420 \\
428\end{array}$ & $\begin{array}{r}10 \\
5\end{array}$ & $\begin{array}{l}-589 \\
-594\end{array}$ & $\begin{array}{l}0 \\
0\end{array}$ \\
\hline $\begin{array}{l}\text { 07N52E03AAAC } \\
\text { O7N53E22DCAC } \\
\text { O7N54E } 14 A D \\
\text { O7NS6E1SDD } \\
\text { 07N5BE2ZB }\end{array}$ & $\begin{array}{l}\text { NPK1-3 } \\
\text { ANDER 1 } \\
\text { HO1-14 } \\
\text { B15-16 } \\
\text { HUETH1 }\end{array}$ & $\begin{array}{l}08 \\
12 \\
11 \\
10 \\
14\end{array}$ & $\begin{array}{l}2963 \\
3120 \\
3035 \\
2737 \\
2993\end{array}$ & & 2952 & 0 & $\begin{array}{l}2707 \\
2687 \\
2752\end{array}$ & $\begin{array}{l}170 \\
140 \\
125\end{array}$ & $\begin{array}{l}2519 \\
2542 \\
2611\end{array}$ & $\begin{array}{r}100 \\
80 \\
150\end{array}$ & $\begin{array}{l}2089 \\
2222 \\
2101 \\
1994\end{array}$ & $\begin{array}{l}225 \\
235 \\
200 \\
185\end{array}$ & $\begin{array}{l}1817 \\
1812 \\
1768 \\
1737\end{array}$ & $\begin{array}{l}0 \\
0 \\
0 \\
0\end{array}$ & $\begin{array}{r}1076 \\
1042 \\
990 \\
924\end{array}$ & $\begin{array}{l}70 \\
60 \\
65 \\
55\end{array}$ & $\begin{array}{r}873 \\
852 \\
815 \\
777 \\
1412\end{array}$ & $\begin{array}{l}0 \\
0 \\
0 \\
0 \\
0\end{array}$ & $\begin{array}{l}486 \\
484 \\
405 \\
347 \\
847\end{array}$ & $\begin{array}{r}5 \\
15 \\
20 \\
0 \\
5\end{array}$ & $\begin{array}{l}-546 \\
-560 \\
-649 \\
-728 \\
-248\end{array}$ & $\begin{array}{l}0 \\
0 \\
0 \\
0 \\
0\end{array}$ \\
\hline $\begin{array}{l}\text { 07NS9E 01BU } \\
\text { 07N59E I DDUAC } \\
\text { O7N60E I OBDBD } \\
\text { O7N61E O5AAC } \\
\text { O6N15E 02BDC }\end{array}$ & $\begin{array}{l}F-11-1 \\
44-12 A \\
A 1920 \\
B N 1 \\
\text { STATEI }\end{array}$ & $\begin{array}{l}11 \\
13 \\
12 \\
13 \\
10\end{array}$ & $\begin{array}{l}2958 \\
2930 \\
2900 \\
3093 \\
4808\end{array}$ & & & & & & & & & & & & & & $\begin{array}{l}2322 \\
1753\end{array}$ & $\begin{array}{l}0 \\
0\end{array}$ & $\begin{array}{l}1740 \\
1746 \\
1184\end{array}$ & $\begin{array}{r}5 \\
10 \\
10\end{array}$ & $\begin{array}{l}653 \\
697 \\
150\end{array}$ & $\begin{array}{l}0 \\
0 \\
0\end{array}$ \\
\hline
\end{tabular}




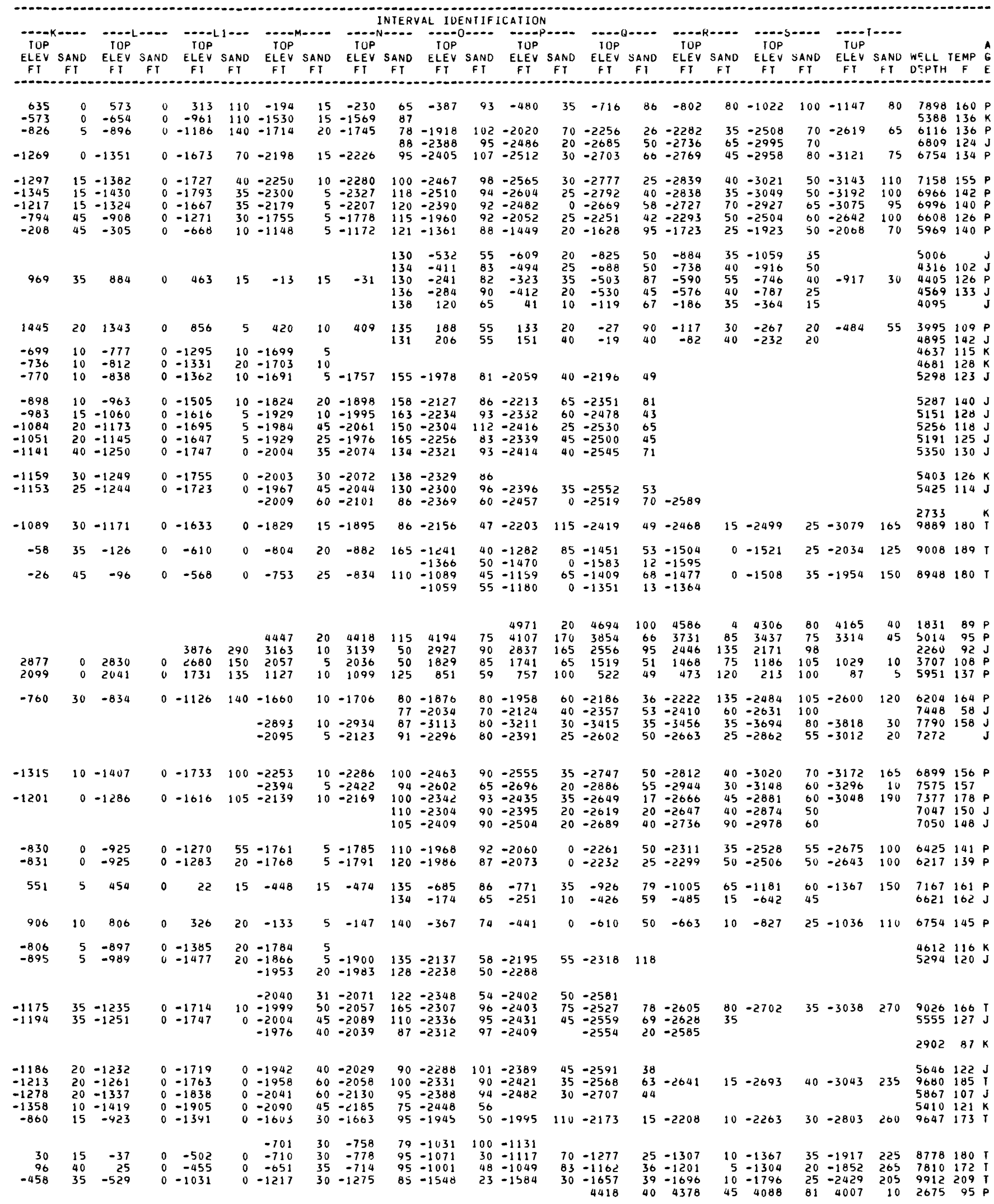


TABLE 2. - SELECTED GEOLUGIC DATA -- CONTINUED

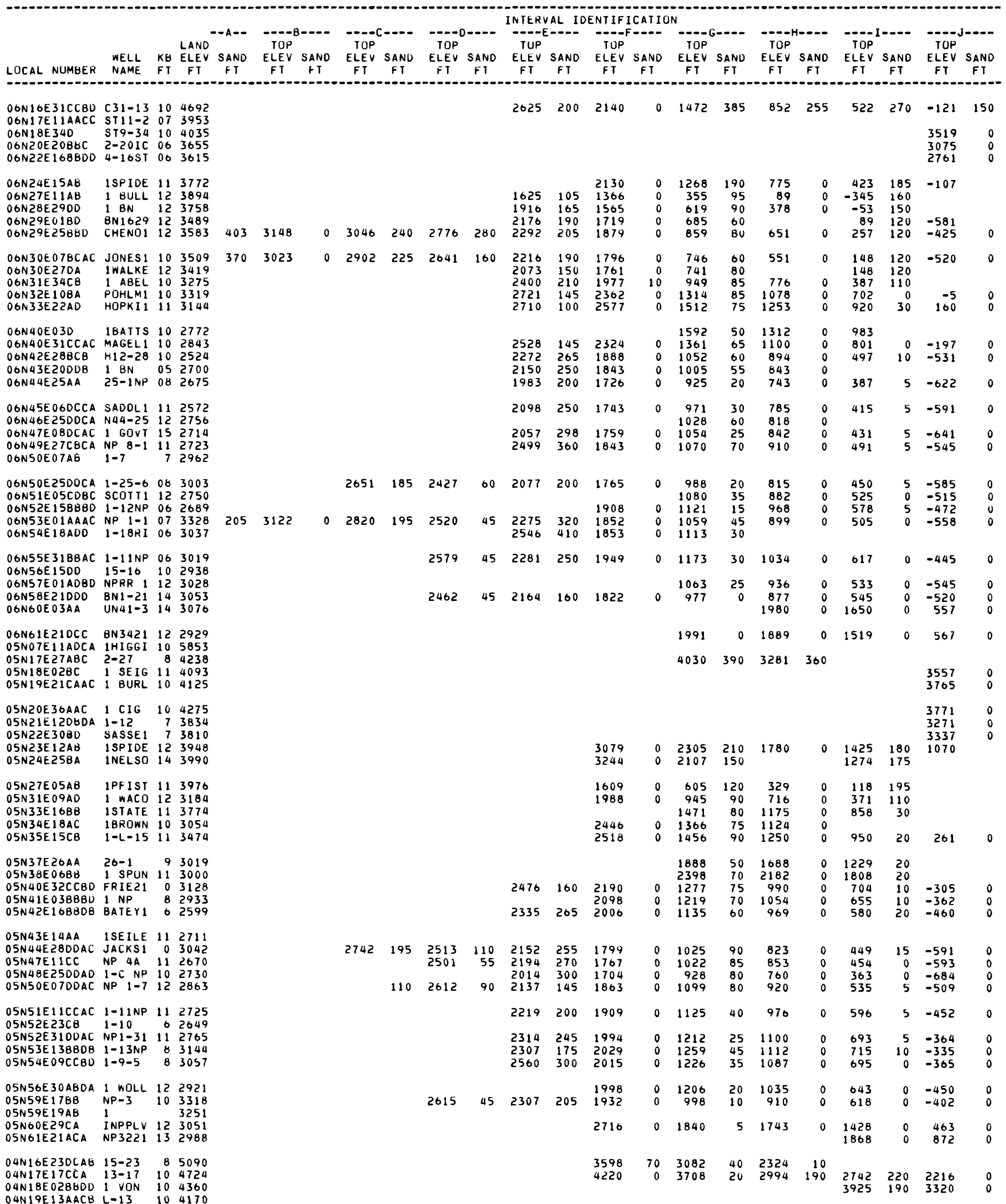




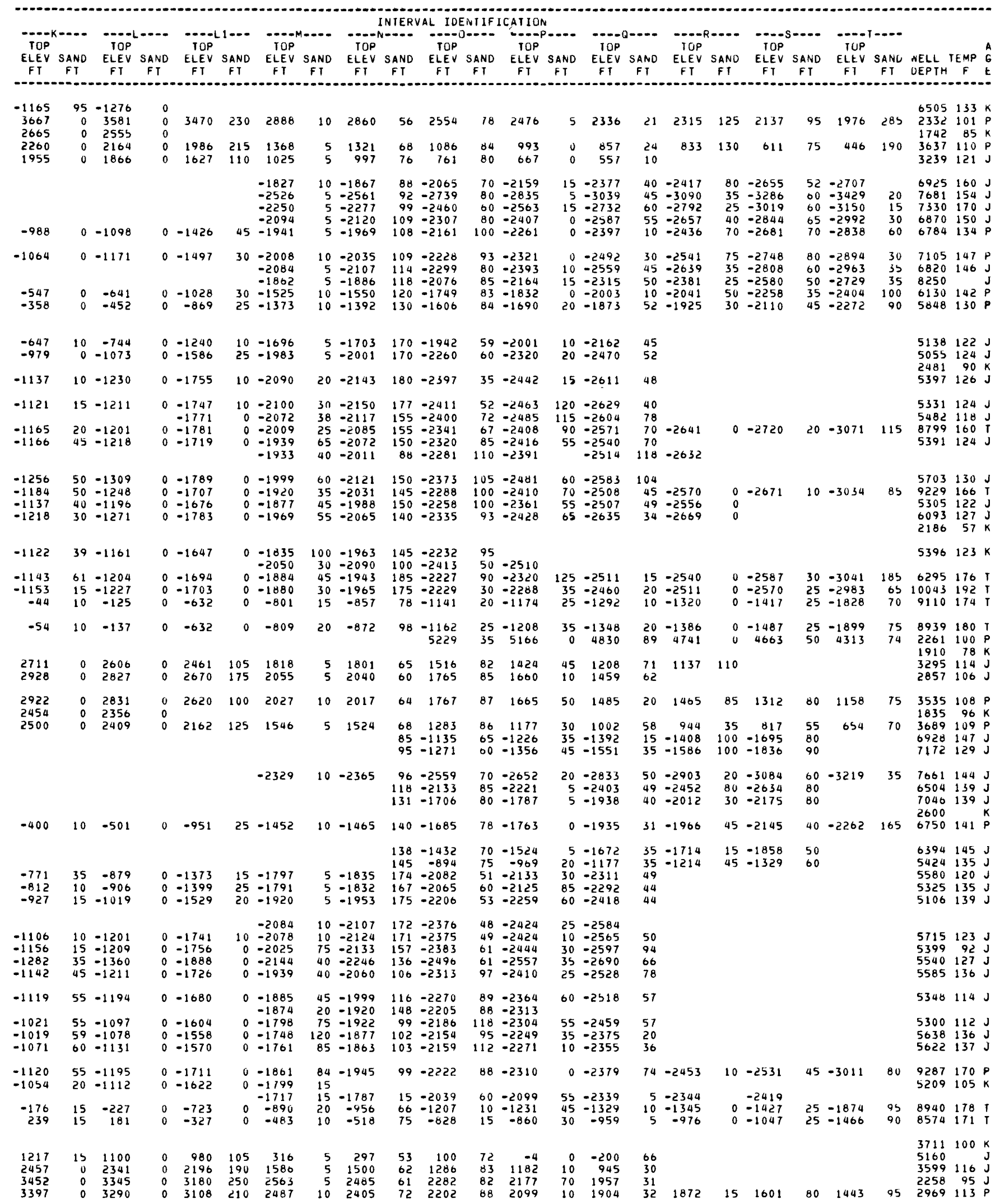




\begin{tabular}{|c|c|c|c|c|c|c|c|c|c|c|c|c|c|c|c|c|c|c|c|c|c|c|}
\hline LOCAL NUMGER & $\begin{array}{l}\text { WELL } \\
\text { NAME }\end{array}$ & $\begin{array}{l}K B \\
F I\end{array}$ & $\begin{array}{c}\text { LAND } \\
\text { ELEV } \\
\text { FT }\end{array}$ & $\begin{array}{l}\text { SAND } \\
\text { FT }\end{array}$ & $\begin{array}{l}\text { TOP } \\
\text { ELEV } \\
\text { FI }\end{array}$ & $\begin{array}{l}\text { SAND } \\
\text { FI }\end{array}$ & $\begin{array}{l}\text { TOP } \\
\text { ELEV } \\
\text { FT }\end{array}$ & $\begin{array}{c}\text { SAND } \\
\text { FI }\end{array}$ & $\begin{array}{l}\text { TOP } \\
\text { ELEV } \\
\text { FT }\end{array}$ & $\begin{array}{l}\text { SAND } \\
\text { FI }\end{array}$ & $\begin{array}{l}-T-E \\
\text { TOP } \\
\text { ELEV }\end{array}$ & $\begin{array}{c}\text { SAND } \\
\text { FT }\end{array}$ & $\begin{array}{l}\text { TOP } \\
\text { ELEV } \\
\text { FT }\end{array}$ & $\begin{array}{c}\text { SAND } \\
\text { FT }\end{array}$ & $\begin{array}{l}\text { TOP } \\
\text { ELEV } \\
\text { FT }\end{array}$ & $\begin{array}{c}\text { SAND } \\
\text { FT }\end{array}$ & $\begin{array}{l}\text { TOP } \\
\text { ELEV } \\
\text { FT }\end{array}$ & $\begin{array}{c}\text { SAND } \\
\text { FI }\end{array}$ & $\begin{array}{l}\text { TOP } \\
\text { ELEV } \\
\text { FI }\end{array}$ & $\begin{array}{c}\text { SAND } \\
\text { FT }\end{array}$ & $\begin{array}{l}\text { TOP } \\
\text { ELEV } \\
\text { FT }\end{array}$ & $\begin{array}{c}\text { SAND } \\
\text { FI }\end{array}$ \\
\hline $\begin{array}{l}\text { O4N2 1E 030ABC } \\
\text { O4N22E } 31 \text { AD } \\
\text { O4N23E } 31 \text { ICADA } \\
\text { O4N24E 25AD } \\
\text { O4N2SE02DA }\end{array}$ & $\begin{array}{l}3-4-21 \\
1 \text { NELD } \\
11-31 \\
1 F O R Q U \\
2-9\end{array}$ & $\begin{array}{r}12 \\
7 \\
10 \\
10 \\
12\end{array}$ & $\begin{array}{l}3895 \\
4165 \\
4041 \\
4020 \\
3927\end{array}$ & & & & & & & & 3487 & 170 & $\begin{array}{l}3230 \\
3133\end{array}$ & $\begin{array}{l}0 \\
0\end{array}$ & $\begin{array}{l}2530 \\
2339\end{array}$ & $\begin{array}{r}130 \\
80\end{array}$ & $\begin{array}{l}2038 \\
2089\end{array}$ & $\begin{array}{l}0 \\
0\end{array}$ & $\begin{array}{l}1594 \\
1515\end{array}$ & $\begin{array}{l}210 \\
210\end{array}$ & $\begin{array}{r}3592 \\
3141 \\
839\end{array}$ & $\begin{array}{l}0 \\
0\end{array}$ \\
\hline $\begin{array}{l}\text { OUN2SE } 11 A D \\
04 N 26 E 17 A B \\
04 N 27 E 26 C A \\
04 N 29 E 36 C C \\
04 N 30 E 07 C D\end{array}$ & $\begin{array}{l}\text { 1CLEVE } \\
\text { NPRR 1 } \\
\text { 1MCKAY } \\
1 \text { TALCO } \\
2 \text { TALCO }\end{array}$ & $\begin{array}{l}11 \\
7 \\
10 \\
12 \\
12\end{array}$ & $\begin{array}{l}3946 \\
3564 \\
3345 \\
3201 \\
3190\end{array}$ & & & & & & & & 2628 & 90 & $\begin{array}{l}3087 \\
3208 \\
2709 \\
2813 \\
2322\end{array}$ & $\begin{array}{l}0 \\
0 \\
0 \\
0 \\
0\end{array}$ & $\begin{array}{l}2339 \\
2471 \\
1837 \\
1813 \\
1277\end{array}$ & $\begin{array}{r}105 \\
70 \\
100 \\
110 \\
90\end{array}$ & $\begin{array}{l}2221 \\
1580 \\
1586 \\
1118\end{array}$ & $\begin{array}{l}0 \\
0 \\
0\end{array}$ & $\begin{array}{l}1517 \\
1649 \\
1135 \\
1184 \\
716\end{array}$ & $\begin{array}{l}180 \\
210 \\
180 \\
120 \\
110\end{array}$ & $\begin{array}{l}941 \\
555\end{array}$ & 0 \\
\hline $\begin{array}{l}\text { OUN33E27BC } \\
04 N 34 E 34 A C \\
04 N 35 E 11 D D \\
04 N 37 E 14 C C \\
\text { OUN39E27BBAC }\end{array}$ & $\begin{array}{l}1 \text { BUYSE } \\
1 \text { KELLE } \\
1-11 \\
1-14 \\
\text { NPRR } 1\end{array}$ & $\begin{array}{l}15 \\
10 \\
11 \\
11 \\
11\end{array}$ & $\begin{array}{l}3002 \\
3100 \\
3411 \\
3049 \\
3369\end{array}$ & & & & & & & & 2408 & 40 & $\begin{array}{l}2352 \\
2427 \\
2442 \\
2313\end{array}$ & $\begin{array}{l}0 \\
0 \\
0 \\
0\end{array}$ & $\begin{array}{l}1607 \\
1385 \\
1457 \\
1580 \\
1362\end{array}$ & $\begin{array}{r}95 \\
100 \\
75 \\
75 \\
25\end{array}$ & $\begin{array}{l}1072 \\
1310 \\
1170\end{array}$ & $\begin{array}{l}0 \\
0 \\
0\end{array}$ & $\begin{array}{r}1022 \\
890 \\
892 \\
998 \\
856\end{array}$ & $\begin{array}{l}30 \\
30 \\
20 \\
20 \\
20\end{array}$ & $\begin{array}{r}117 \\
-93\end{array}$ & 0 \\
\hline $\begin{array}{l}\text { OUN } 42 E 22 D D B D \\
04 N 43 E 28 A A B D \\
04 N 44 E 34 C C A C \\
04 N 45 E 13 A A \\
04 N 45 E 32 D D C A\end{array}$ & $\begin{array}{l}16-22 \\
1 \text { SUMM } \\
\text { LUND } 1 \\
\text { ISONAI } \\
1 \text { TURN }\end{array}$ & $\begin{array}{l}5 \\
10 \\
11 \\
11 \\
11\end{array}$ & $\begin{array}{l}2714 \\
2841 \\
3170 \\
3001 \\
2991\end{array}$ & 230 & 2891 & 20 & $\begin{array}{l}2486 \\
2704 \\
\\
2703\end{array}$ & $\begin{array}{l}140 \\
120 \\
75\end{array}$ & $\begin{array}{l}2304 \\
2431 \\
2380\end{array}$ & $\begin{array}{l}30 \\
35\end{array}$ & $\begin{array}{l}2201 \\
2121 \\
2157 \\
2002 \\
1963\end{array}$ & $\begin{array}{l}140 \\
205 \\
100 \\
170 \\
140\end{array}$ & $\begin{array}{l}1901 \\
1790 \\
1791 \\
1714 \\
1702\end{array}$ & $\begin{array}{l}0 \\
0 \\
0 \\
0 \\
0\end{array}$ & $\begin{array}{r}1059 \\
982 \\
987 \\
905 \\
879\end{array}$ & $\begin{array}{l}60 \\
70 \\
80 \\
80 \\
75\end{array}$ & $\begin{array}{l}797 \\
785 \\
720 \\
696\end{array}$ & $\begin{array}{l}0 \\
0 \\
0 \\
0\end{array}$ & $\begin{array}{l}417 \\
421 \\
348 \\
344\end{array}$ & $\begin{array}{l}15 \\
15 \\
10 \\
10\end{array}$ & $\begin{array}{l}-649 \\
-509 \\
-696 \\
-726\end{array}$ & $\begin{array}{l}0 \\
0 \\
0 \\
0\end{array}$ \\
\hline 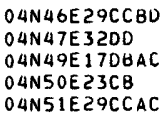 & $\begin{array}{l}\text { NP 20 } \\
\text { PYLE } 1 \\
10-17 \\
1=27 \\
1=3\end{array}$ & $\begin{array}{r}11 \\
7 \\
8 \\
11\end{array}$ & $\begin{array}{l}2841 \\
2652 \\
2969 \\
2825 \\
2740\end{array}$ & & & & & & 2432 & 70 & $\begin{array}{l}2018 \\
2071 \\
1983 \\
2370\end{array}$ & $\begin{array}{r}190 \\
155 \\
35 \\
265\end{array}$ & $\begin{array}{l}1664 \\
1844 \\
1925 \\
1919\end{array}$ & $\begin{array}{l}0 \\
0 \\
0\end{array}$ & $\begin{array}{l}1136 \\
1135\end{array}$ & $\begin{array}{l}60 \\
25\end{array}$ & $\begin{array}{r}1009 \\
988\end{array}$ & $\begin{array}{l}0 \\
0\end{array}$ & $\begin{array}{l}613 \\
623\end{array}$ & $\begin{array}{l}0 \\
0\end{array}$ & $\begin{array}{l}-422 \\
-425\end{array}$ & $\begin{array}{l}0 \\
0\end{array}$ \\
\hline $\begin{array}{l}\text { OUN52E3 } 28 B C A \\
\text { O4N53E01BBBD } \\
04 N 54 E 29 B O C A \\
04 N 55 E 23 A A \\
\text { O4N56E21BB }\end{array}$ & $\begin{array}{l}11-34 \\
s-1-1 \\
1-29 \\
1-7 \\
1-8\end{array}$ & $\begin{array}{r}10 \\
6 \\
8 \\
6 \\
6\end{array}$ & $\begin{array}{l}2855 \\
2697 \\
2714 \\
3155 \\
2940\end{array}$ & & & & & & & & $\begin{array}{l}2373 \\
2338 \\
2599\end{array}$ & $\begin{array}{l}145 \\
120 \\
240\end{array}$ & $\begin{array}{l}2060 \\
2055 \\
2097 \\
2191\end{array}$ & $\begin{array}{l}0 \\
0 \\
0 \\
0\end{array}$ & $\begin{array}{l}1271 \\
1258 \\
1316 \\
1409\end{array}$ & $\begin{array}{l}40 \\
40 \\
30 \\
30\end{array}$ & $\begin{array}{l}1149 \\
1131 \\
1189 \\
1273\end{array}$ & $\begin{array}{l}0 \\
0 \\
0 \\
0\end{array}$ & $\begin{array}{l}761 \\
731 \\
788 \\
883\end{array}$ & $\begin{array}{l}5 \\
0 \\
5 \\
0\end{array}$ & $\begin{array}{l}-305 \\
-330 \\
-288 \\
-186\end{array}$ & $\begin{array}{l}0 \\
0 \\
0 \\
0\end{array}$ \\
\hline $\begin{array}{l}\text { 04N57E } 1 \text { 1BBAC } \\
04 N 59 E 07 A A \\
04 N 60 E 21 D C C \\
04 N 61 E 01 C D D B \\
04 N 61 E 030 B A\end{array}$ & $\begin{array}{l}1-9 \\
\text { NPRR } 5 \\
1 \\
24 x-1 \\
33=3\end{array}$ & $\begin{array}{r}6 \\
10 \\
8 \\
12 \\
7\end{array}$ & $\begin{array}{l}3043 \\
3345 \\
3165 \\
3060 \\
3051\end{array}$ & & & & & & 2630 & 20 & $\begin{array}{l}2495 \\
2229\end{array}$ & $\begin{array}{l}195 \\
205\end{array}$ & $\begin{array}{l}2159 \\
1910\end{array}$ & $\begin{array}{l}0 \\
0\end{array}$ & $\begin{array}{l}1359 \\
1155\end{array}$ & $\begin{array}{l}0 \\
0\end{array}$ & $\begin{array}{r}1203 \\
935\end{array}$ & $\begin{array}{l}0 \\
0\end{array}$ & $\begin{array}{l}823 \\
598\end{array}$ & $\begin{array}{l}0 \\
0\end{array}$ & $\begin{array}{r}-274 \\
-492 \\
787\end{array}$ & $\begin{array}{l}0 \\
0\end{array}$ \\
\hline $\begin{array}{l}\text { 04N62E 17BAAC } \\
03 N 15 E 32 C B A C \\
03 N 18 E 08 A A A C \\
03 N 18 E 27 A B \\
03 N 19 E 070 A \\
\text { 03N }\end{array}$ & $\begin{array}{l}21-17 \\
13-32 \\
1 B R \\
2-27 C R \\
1 K I M B A\end{array}$ & $\begin{array}{r}13 \\
10 \\
11 \\
7 \\
12\end{array}$ & $\begin{array}{l}2954 \\
4490 \\
4872 \\
4583 \\
4394\end{array}$ & & & & 3298 & 415 & 2420 & 35 & 2245 & 280 & 1912 & 90 & $\begin{array}{l}1468 \\
4545 \\
4287\end{array}$ & $\begin{array}{l}410 \\
320\end{array}$ & $\begin{array}{r}762 \\
3870 \\
3540\end{array}$ & $\begin{array}{l}230 \\
265\end{array}$ & 3453 & 175 & $\begin{array}{r}863 \\
2768\end{array}$ & 0 \\
\hline $\begin{array}{l}\text { O3N20E01BA } \\
03 N 20 E 320 \\
03 N 21 E 36 C C C A \\
03 N 22 E 36 C \\
\text { O3N23E } 180 B B\end{array}$ & $\begin{array}{l}\text { IMITCH } \\
9-32 \\
13-36 \\
23-30 \\
\text { ISTELT }\end{array}$ & $\begin{array}{r}12 \\
10 \\
9 \\
9 \\
8\end{array}$ & $\begin{array}{l}4064 \\
4050 \\
4056 \\
4206 \\
3992\end{array}$ & & & & & & & & & & & & 3955 & 270 & $\begin{array}{l}3255 \\
4038\end{array}$ & $\begin{array}{r}235 \\
70\end{array}$ & $\begin{array}{l}3440 \\
3602\end{array}$ & $\begin{array}{l}180 \\
210\end{array}$ & $\begin{array}{l}2644 \\
2980\end{array}$ & $\begin{array}{l}0 \\
0\end{array}$ \\
\hline $\begin{array}{l}03 N 26 E 33 B A \\
03 N 31 E 030 B \\
03 N 31 E 08 D B \\
03 N 31 E 28 B C A C \\
03 N 32 E 26 B B\end{array}$ & $\begin{array}{l}1 \text { MACK } \\
33-8 P 1 \\
1 \text { KENDR } \\
1 \text { GEAR } \\
1 \text { DOSS }\end{array}$ & $\begin{array}{r}12 \\
10 \\
10 \\
9 \\
11\end{array}$ & $\begin{array}{l}3353 \\
2845 \\
2845 \\
3018 \\
3266\end{array}$ & & & & & & & & & & $\begin{array}{l}2757 \\
2797\end{array}$ & $\begin{array}{l}10 \\
10\end{array}$ & $\begin{array}{l}1932 \\
1862\end{array}$ & $\begin{array}{l}100 \\
100\end{array}$ & $\begin{array}{l}2572 \\
1636 \\
1577\end{array}$ & $\begin{array}{l}0 \\
0\end{array}$ & $\begin{array}{l}2276 \\
1347 \\
1296\end{array}$ & $\begin{array}{l}45 \\
30\end{array}$ & $\begin{array}{l}1577 \\
\\
735 \\
627\end{array}$ & $\begin{array}{l}0 \\
0\end{array}$ \\
\hline $\begin{array}{l}\text { O3N33E13BA } \\
03 N 34 E 36 C C A \\
03 N 35 E 32 A D A B \\
03 N 39 E 29 A C \\
\text { O3N4OE } 18 A C B D\end{array}$ & $\begin{array}{l}\text { IVAN C } \\
A-1 \\
1-32 \\
1=29 \\
1-18\end{array}$ & $\begin{array}{r}11 \\
4 \\
10 \\
10 \\
10\end{array}$ & $\begin{array}{l}2938 \\
3103 \\
3379 \\
3204 \\
2958\end{array}$ & & & & 2763 & 90 & $\begin{array}{l}2898 \\
2974 \\
2494 \\
2752\end{array}$ & $\begin{array}{r}90 \\
50 \\
100 \\
95\end{array}$ & $\begin{array}{l}2612 \\
2568 \\
2119 \\
2338\end{array}$ & $\begin{array}{r}100 \\
80 \\
95 \\
130\end{array}$ & $\begin{array}{l}2409 \\
2317 \\
1894 \\
2109\end{array}$ & $\begin{array}{r}20 \\
0 \\
10 \\
0\end{array}$ & $\begin{array}{l}1467 \\
1362 \\
1041 \\
1193\end{array}$ & $\begin{array}{r}100 \\
85 \\
40 \\
35\end{array}$ & $\begin{array}{r}1208 \\
1127 \\
794 \\
966\end{array}$ & $\begin{array}{l}5 \\
0 \\
5 \\
5\end{array}$ & $\begin{array}{l}849 \\
524 \\
682\end{array}$ & $\begin{array}{l}30 \\
35 \\
30\end{array}$ & $\begin{array}{r}131 \\
-426 \\
-292\end{array}$ & $\begin{array}{l}0 \\
0 \\
0\end{array}$ \\
\hline $\begin{array}{l}03 N 40 E 23 D C \\
03 N 41 E 130 D B D \\
03 N 42 E 12 A B D B \\
03 N 43 E 32 A C \\
03 N 44 E 1300 \\
030\end{array}$ & $\begin{array}{l}N P 1 \\
1-13 \\
1 \text { GOUT } \\
1-32 \\
N P 19\end{array}$ & $\begin{array}{r}9 \\
0 \\
10 \\
12\end{array}$ & $\begin{array}{l}3365 \\
3201 \\
2831 \\
2834 \\
3080\end{array}$ & & & & 2830 & 85 & $\begin{array}{l}2450 \\
2591 \\
2584 \\
2589\end{array}$ & $\begin{array}{l}100 \\
110 \\
100 \\
140\end{array}$ & $\begin{array}{l}2167 \\
2157 \\
2344 \\
2252\end{array}$ & $\begin{array}{l}225 \\
170 \\
210 \\
240\end{array}$ & $\begin{array}{l}1760 \\
1820 \\
1716 \\
1762\end{array}$ & $\begin{array}{r}0 \\
0 \\
10 \\
0\end{array}$ & $\begin{array}{r}939 \\
1038 \\
959 \\
981\end{array}$ & $\begin{array}{l}90 \\
80 \\
90 \\
75\end{array}$ & $\begin{array}{l}725 \\
798 \\
717 \\
752\end{array}$ & $\begin{array}{l}10 \\
10 \\
40 \\
10\end{array}$ & $\begin{array}{l}463 \\
430\end{array}$ & $\begin{array}{l}15 \\
15\end{array}$ & $\begin{array}{l}-557 \\
-588\end{array}$ & 0 \\
\hline $\begin{array}{l}03 N 45 E 23 B B \\
03 N 46 E 03 C B A C \\
03 N 47 E 220 B B D \\
03 N 48 E 27 A A \\
03 N 50 E 09 B A C A \\
03 N 50\end{array}$ & $\begin{array}{l}1 \text { NP } \\
1 \text { POWE } \\
22347 \\
\text { NP } 2-A \\
1-9\end{array}$ & $\begin{array}{r}0 \\
10 \\
9 \\
11 \\
10\end{array}$ & $\begin{array}{l}2927 \\
2740 \\
2863 \\
3067 \\
2798\end{array}$ & & & & $\begin{array}{l}2711 \\
2637 \\
2828\end{array}$ & $\begin{array}{r}80 \\
135 \\
100\end{array}$ & $\begin{array}{l}2453 \\
2450 \\
2467 \\
2543 \\
2399\end{array}$ & $\begin{array}{r}200 \\
80 \\
85 \\
100 \\
30\end{array}$ & $\begin{array}{l}2057 \\
2055 \\
2219 \\
1948 \\
2218\end{array}$ & $\begin{array}{l}130 \\
220 \\
360 \\
130 \\
115\end{array}$ & $\begin{array}{l}1663 \\
1658 \\
1685 \\
1773 \\
1906\end{array}$ & $\begin{array}{l}0 \\
0 \\
0 \\
0 \\
0\end{array}$ & $\begin{array}{r}891 \\
910 \\
952 \\
1016 \\
1148\end{array}$ & $\begin{array}{l}70 \\
90 \\
80 \\
55 \\
40\end{array}$ & $\begin{array}{l}669 \\
682 \\
733 \\
838 \\
988\end{array}$ & $\begin{array}{r}10 \\
5 \\
5 \\
0 \\
0\end{array}$ & $\begin{array}{l}349 \\
365 \\
432 \\
509 \\
623\end{array}$ & $\begin{array}{r}10 \\
0 \\
0 \\
0 \\
0\end{array}$ & $\begin{array}{l}-663 \\
-680 \\
-623 \\
-537 \\
-417\end{array}$ & $\begin{array}{r}0 \\
0 \\
20 \\
0 \\
0\end{array}$ \\
\hline $\begin{array}{l}\text { 03N52E 25BD } \\
\text { 03N53E 23AABD } \\
03 N 54 E 23 A A \\
03 N 55 E 27 A A \\
\text { O3N57E 07BAAC }\end{array}$ & $\begin{array}{l}1 \text { DANG } \\
1-23 \\
1-23 \\
1-27 \\
1-6\end{array}$ & $\begin{aligned} 10 \\
10 \\
10 \\
10 \\
0\end{aligned}$ & $\begin{array}{l}2937 \\
2808 \\
2785 \\
3178 \\
3044\end{array}$ & & & & & & 2666 & 50 & $\begin{array}{l}2509 \\
2438 \\
2836 \\
2687\end{array}$ & $\begin{array}{l}190 \\
170 \\
240 \\
120\end{array}$ & $\begin{array}{l}2097 \\
2139 \\
2245 \\
2327 \\
2289\end{array}$ & $\begin{array}{l}0 \\
0 \\
0 \\
0 \\
0\end{array}$ & $\begin{array}{l}1351 \\
1403 \\
1485 \\
1561 \\
1470\end{array}$ & $\begin{array}{r}30 \\
30 \\
25 \\
10 \\
0\end{array}$ & $\begin{array}{l}1197 \\
1240 \\
1323 \\
1401 \\
1320\end{array}$ & $\begin{array}{l}0 \\
0 \\
0 \\
0 \\
0\end{array}$ & $\begin{array}{r}871 \\
948 \\
1030 \\
952\end{array}$ & $\begin{array}{r}0 \\
0 \\
10 \\
0\end{array}$ & $\begin{array}{r}-161 \\
-85 \\
-1 \\
-132\end{array}$ & $\begin{array}{l}0 \\
0 \\
0 \\
0\end{array}$ \\
\hline $\begin{array}{l}\text { O3N59E 19AC } \\
02 N 13 E 34 C E C \\
02 N 13 E 36 D B \\
02 N 15 E 23 A A C C \\
02 N 17 E 04 B D D C\end{array}$ & $\begin{array}{l}32-19 \\
10 B U R N \\
1 B I G I I \\
1-23 \\
1 \text { STAT }\end{array}$ & $\begin{array}{l}11 \\
17 \\
14 \\
12 \\
11\end{array}$ & $\begin{array}{l}3198 \\
4751 \\
4535 \\
4574 \\
4951\end{array}$ & & & & 4050 & 205 & $\begin{array}{l}3471 \\
4042\end{array}$ & $\begin{array}{r}150 \\
15\end{array}$ & $\begin{array}{l}3036 \\
3901\end{array}$ & $\begin{array}{l}200 \\
195\end{array}$ & $\begin{array}{l}2711 \\
3547\end{array}$ & $\begin{array}{l}25 \\
25\end{array}$ & $\begin{array}{l}2516 \\
3162\end{array}$ & $\begin{array}{l}390 \\
350\end{array}$ & $\begin{array}{l}1648 \\
2407\end{array}$ & $\begin{array}{l}310 \\
525\end{array}$ & $\begin{array}{r}817 \\
1612\end{array}$ & $\begin{array}{l}150 \\
150\end{array}$ & $\begin{array}{r}296 \\
1006\end{array}$ & $\begin{array}{l}0 \\
0\end{array}$ \\
\hline
\end{tabular}




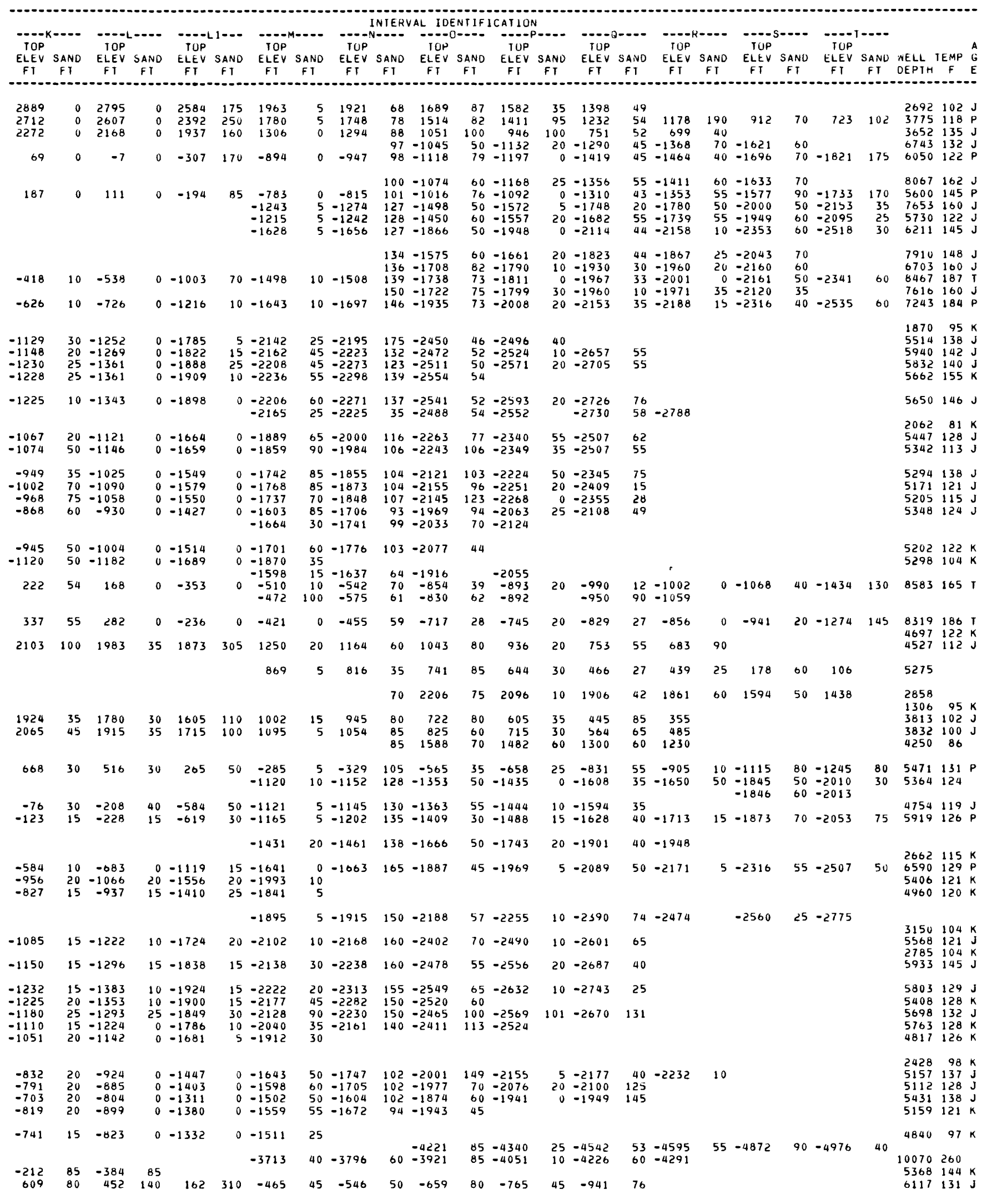


TABLE 2. - SELECTEO GEOLOGIC DATA -- CONTINUED

\begin{tabular}{|c|c|c|c|c|c|c|c|c|c|c|c|c|c|c|c|c|c|c|c|c|c|c|}
\hline LOCAL NUMBER & $\begin{array}{l}\text { WELL } \\
\text { NAME }\end{array}$ & $\begin{array}{l}K B \\
F T\end{array}$ & $\begin{array}{l}\text { LAND } \\
\text { ELEV } \\
\text { FT }\end{array}$ & $\begin{array}{l}-A-- \\
\text { SAND } \\
\text { FT }\end{array}$ & $\begin{array}{l}-P O P \\
\text { ELEV } \\
F T\end{array}$ & $\begin{array}{l}B-D \\
\text { SAND } \\
\text { FT }\end{array}$ & $\begin{array}{c}\text { TOP } \\
\text { ELEV } \\
F T\end{array}$ & SAND & $\begin{array}{l}\text { TOP } \\
\text { ELEV } \\
F T\end{array}$ & $\begin{array}{l}\text { SAND } \\
\text { FT }\end{array}$ & $\begin{array}{l}\text { TOP } \\
\text { ELEV } \\
\text { FI }\end{array}$ & $\begin{array}{l}\text { SAND } \\
\text { FT }\end{array}$ & $\begin{array}{c}\text { TOP } \\
\text { ELEV } \\
\text { FT }\end{array}$ & $\begin{array}{l}\text { SAND } \\
\text { F T }\end{array}$ & $\begin{array}{l}\text { TOP } \\
\text { ELEV } \\
\text { FT }\end{array}$ & $\begin{array}{c}\text { SAND } \\
\text { FT }\end{array}$ & $\begin{array}{l}\text { TOP } \\
\text { ELEV } \\
\text { FT }\end{array}$ & $\begin{array}{c}\text { SAND } \\
\text { F T }\end{array}$ & $\begin{array}{l}-O-I \\
\text { TUP } \\
\text { ELEV } \\
\text { FT }\end{array}$ & $\begin{array}{c}\text { FT } \\
\text { SAND } \\
\text { FT }\end{array}$ & $\begin{array}{l}\text { TOP } \\
\text { ELEV } \\
\text { FT }\end{array}$ & $\begin{array}{l}\text { JEA } \\
\text { SAND } \\
\text { FT }\end{array}$ \\
\hline $\begin{array}{l}\text { O2N19E01CA } \\
\text { OZN19E03BB } \\
02 N 20 E 04 C A \\
\text { OZNZOEOQC } \\
\text { OZNZOE1TAACC }\end{array}$ & $\begin{array}{l}11-1 \\
1 \text { CHRIS } \\
\text { KIRSC } 1 \\
1 M I L L E \\
1 x-17\end{array}$ & $\begin{array}{r}10 \\
11 \\
12 \\
9 \\
12\end{array}$ & $\begin{array}{l}4123 \\
4151 \\
4009 \\
4016 \\
4033\end{array}$ & & & & & & & & & & & & $\begin{array}{l}3576 \\
3600\end{array}$ & $\begin{array}{l}60 \\
60\end{array}$ & $\begin{array}{l}3224 \\
3418 \\
3413 \\
3455 \\
3283\end{array}$ & $\begin{array}{r}240 \\
0 \\
0 \\
0 \\
185\end{array}$ & $\begin{array}{l}2928 \\
2941 \\
2981 \\
3013 \\
2987\end{array}$ & $\begin{array}{l}190 \\
200 \\
190 \\
180 \\
180\end{array}$ & $\begin{array}{l}2096 \\
2181 \\
2127\end{array}$ & 0 \\
\hline $\begin{array}{l}\text { 02N20E29BB } \\
02 N 21 E 18 D B \\
02 N 21 E 22 A A B \\
02 N 21 E 220 B \\
02 N 23 E 24 B A C C\end{array}$ & $\begin{array}{l}\text { GEE 1 } \\
1 \text { COPUL } \\
71-22 \\
1 \text { CAST } \\
22-24\end{array}$ & $\begin{array}{r}9 \\
11 \\
10 \\
9 \\
9\end{array}$ & $\begin{array}{l}4098 \\
3947 \\
3990 \\
4070 \\
4016\end{array}$ & & & & & & & & & & & & $\begin{array}{l}3428 \\
3929\end{array}$ & $\begin{array}{r}70 \\
295\end{array}$ & $\begin{array}{l}3284 \\
3319 \\
3775\end{array}$ & $\begin{array}{r}110 \\
30\end{array}$ & $\begin{array}{l}2878 \\
3112 \\
3447\end{array}$ & $\begin{array}{l}180 \\
190 \\
200\end{array}$ & $\begin{array}{l}2237 \\
2604\end{array}$ & $\begin{array}{l}0 \\
0\end{array}$ \\
\hline $\begin{array}{l}\text { O2N2SE 1OCC } \\
02 N 26 E 01 \text { IA } \\
02 N 2 B E 36 C D B B \\
02 N 29 E 31 C C A D \\
\text { O2N3OEO3CC }\end{array}$ & $\begin{array}{l}\text { STATEI } \\
\text { IDAVIS } \\
1-36 \\
1-A \\
\text { CROW } 1\end{array}$ & $\begin{array}{r}17 \\
7 \\
9 \\
10\end{array}$ & $\begin{array}{l}3592 \\
3231 \\
3446 \\
3452 \\
2974\end{array}$ & & & & & & & & & & & & & & & & $\begin{array}{l}2933 \\
2704\end{array}$ & $\begin{array}{r}105 \\
80\end{array}$ & $\begin{array}{l}2275 \\
1956\end{array}$ & $\begin{array}{l}0 \\
0\end{array}$ \\
\hline $\begin{array}{l}\text { O2N3OE2ZCBCA } \\
02 N 31 \text { IEOADAC } \\
02 N 33 E 35 C D A \\
02 N 34 E 02 C A C D \\
\text { O2N34E } 22 A D A\end{array}$ & $\begin{array}{l}1 \text { LIND } \\
1 \text { FELT } \\
1 \text { WEINB } \\
1 \text { WOLF } \\
1\end{array}$ & $\begin{array}{r}10 \\
10 \\
12 \\
6\end{array}$ & $\begin{array}{l}3016 \\
3196 \\
2818 \\
3122 \\
3117\end{array}$ & & & & & & & & 2738 & 140 & 2471 & 35 & $\begin{array}{l}2435 \\
2012 \\
1560\end{array}$ & $\begin{array}{l}110 \\
100 \\
100\end{array}$ & $\begin{array}{l}2166 \\
1853 \\
1268\end{array}$ & $\begin{array}{r}10 \\
5 \\
0\end{array}$ & 1524 & 40 & 893 & 0 \\
\hline 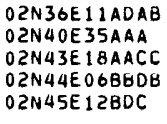 & $\begin{array}{l}1 \text { DERR } \\
35-1 \\
41-18 \\
\text { SPRING } \\
1\end{array}$ & $\begin{array}{r}12 \\
4 \\
4 \\
11 \\
6\end{array}$ & $\begin{array}{l}3277 \\
3326 \\
2926 \\
3075 \\
2730\end{array}$ & 170 & 3070 & 70 & $\begin{array}{l}2850 \\
2757 \\
2796\end{array}$ & $\begin{array}{r}180 \\
120 \\
90\end{array}$ & $\begin{array}{l}2669 \\
2520 \\
2547 \\
2574\end{array}$ & $\begin{array}{l}50 \\
100 \\
143 \\
120\end{array}$ & $\begin{array}{l}2437 \\
2215 \\
2119 \\
2151\end{array}$ & $\begin{array}{r}90 \\
160 \\
200 \\
145\end{array}$ & $\begin{array}{l}2149 \\
1858 \\
1644 \\
1686\end{array}$ & $\begin{array}{l}0 \\
0 \\
0 \\
0\end{array}$ & $\begin{array}{r}1248 \\
1015 \\
900 \\
935\end{array}$ & $\begin{array}{l}90 \\
75 \\
90 \\
70\end{array}$ & $\begin{array}{r}1018 \\
840 \\
657 \\
702\end{array}$ & $\begin{array}{r}0 \\
20 \\
10 \\
5\end{array}$ & 689 & 50 & $\begin{array}{l}-61 \\
-686\end{array}$ & 0 \\
\hline $\begin{array}{l}02 N 45 E 150 C B O \\
02 N 46 E 3500 \\
02 N 47 E 196 B B D \\
02 N 48 E 35 A A B D \\
02 N 49 E 23 D D B D\end{array}$ & $\begin{array}{l}34-15 \\
N P \text { I-A } \\
1 \text { NP } \\
2 \text { ALLE } \\
1-23\end{array}$ & $\begin{array}{r}11 \\
11 \\
0 \\
12 \\
9\end{array}$ & $\begin{array}{l}2770 \\
3057 \\
2954 \\
2986 \\
3199\end{array}$ & & & & $\begin{array}{l}2668 \\
2720\end{array}$ & $\begin{array}{l}200 \\
130\end{array}$ & $\begin{array}{l}2492 \\
2273 \\
2453 \\
2488 \\
2783\end{array}$ & $\begin{array}{r}140 \\
70 \\
135 \\
110 \\
145\end{array}$ & $\begin{array}{l}2021 \\
2088 \\
2131 \\
2083 \\
2325\end{array}$ & $\begin{array}{l}180 \\
180 \\
165 \\
140 \\
150\end{array}$ & $\begin{array}{l}1635 \\
1553 \\
1634 \\
1748 \\
1893\end{array}$ & $\begin{array}{l}0 \\
0 \\
0 \\
0 \\
0\end{array}$ & $\begin{array}{r}901 \\
837 \\
876 \\
1005 \\
1158\end{array}$ & $\begin{array}{l}60 \\
70 \\
65 \\
50 \\
30\end{array}$ & $\begin{array}{l}678 \\
621 \\
661 \\
822 \\
978\end{array}$ & $\begin{array}{l}5 \\
5 \\
5 \\
0 \\
5\end{array}$ & $\begin{array}{l}329 \\
373 \\
538 \\
676\end{array}$ & $\begin{array}{l}0 \\
0 \\
0 \\
0\end{array}$ & $\begin{array}{l}-682 \\
-629 \\
-477 \\
-336\end{array}$ & $\begin{array}{l}0 \\
0 \\
0 \\
0\end{array}$ \\
\hline $\begin{array}{l}\text { O2N5OE1GCAAC } \\
02 N 51 E 21 A A \\
02 N 51 E 360 C B C \\
02 N 52 E 20 C B \\
02 N 53 E 2 B A A\end{array}$ & $\begin{array}{l}23-16 \\
21-1 \\
1 \text { STAT } \\
\text { GOVT } 1 \\
1-28\end{array}$ & $\begin{array}{l}9 \\
10 \\
12 \\
10 \\
10\end{array}$ & $\begin{array}{l}3165 \\
2934 \\
3007 \\
3275 \\
3103\end{array}$ & & & & & & 2767 & 130 & 2295 & 110 & 1889 & 0 & $\begin{array}{l}1149 \\
1277 \\
1438\end{array}$ & $\begin{array}{l}25 \\
15 \\
15\end{array}$ & $\begin{array}{r}974 \\
1124 \\
1293\end{array}$ & 0 & $\begin{array}{l}789 \\
934\end{array}$ & 0 & $\begin{array}{l}-232 \\
-100\end{array}$ & 0 \\
\hline $\begin{array}{l}\text { O2N5 4E O } 9 B B D B \\
02 N 54 E 230 D \\
02 N 55 E 13 C D \\
02 N 55 E 29 A A \\
02 N 56 E 160 D\end{array}$ & $\begin{array}{l}1-4 \\
1 N P \\
1-2 \\
S-29-2 \\
1 \text { STAT }\end{array}$ & 10 & $\begin{array}{l}2752 \\
2878 \\
3333 \\
3007 \\
3339\end{array}$ & & & & & & & & 3009 & 175 & 2250 & 0 & $\begin{array}{l}1487 \\
1788\end{array}$ & 15 & $\begin{array}{l}1328 \\
1634\end{array}$ & 0 & $\begin{array}{r}964 \\
1264\end{array}$ & 10 & $\begin{array}{l}-70 \\
212\end{array}$ & 0 \\
\hline $\begin{array}{l}\text { O2NSTE } 16 C D \\
02 N 59 E 23 A B \\
02 N 60 E 22 B \$ \\
02 N 61 E 05 C C \\
\text { OIN15E } 33 B C\end{array}$ & $\begin{array}{ll}1 & \\
1 & -23 \\
1 & \text { JARD } \\
1 & \text { TRAW } \\
1 & \text { SHAY }\end{array}$ & $\begin{array}{r}7 \\
7 \\
12 \\
12\end{array}$ & $\begin{array}{l}3514 \\
3517 \\
3220 \\
3167 \\
4103\end{array}$ & & & & & & $\begin{array}{l}2877 \\
3012\end{array}$ & $\begin{array}{l}35 \\
50\end{array}$ & $\begin{array}{l}2544 \\
2681\end{array}$ & $\begin{array}{l}115 \\
150\end{array}$ & $\begin{array}{l}2177 \\
2290\end{array}$ & $\begin{array}{l}0 \\
0\end{array}$ & $\begin{array}{l}1322 \\
1446 \\
2575\end{array}$ & $\begin{array}{r}0 \\
0 \\
60\end{array}$ & $\begin{array}{l}1252 \\
1356 \\
2407\end{array}$ & $\begin{array}{l}0 \\
0 \\
0\end{array}$ & $\begin{array}{r}912 \\
1017 \\
2085\end{array}$ & $\begin{array}{r}0 \\
0 \\
130\end{array}$ & $\begin{array}{r}-193 \\
-61 \\
1339\end{array}$ & $\begin{array}{l}0 \\
0\end{array}$ \\
\hline 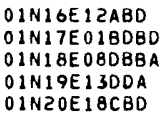 & $\begin{array}{l}1 \text {-PEDE } \\
1 \text {-ADK } 1 \\
1 \text {-SVEN } \\
1 \text { PELTO } \\
1-Z \text { IND }\end{array}$ & $\begin{array}{r}8 \\
10 \\
12 \\
11 \\
8\end{array}$ & $\begin{array}{l}5052 \\
5072 \\
4994 \\
4930 \\
4781\end{array}$ & & & & $\begin{array}{l}4670 \\
4566 \\
4514\end{array}$ & $\begin{array}{l}210 \\
435 \\
305\end{array}$ & $\begin{array}{l}4095 \\
3917 \\
3916 \\
4419\end{array}$ & $\begin{array}{r}150 \\
30 \\
70 \\
10\end{array}$ & $\begin{array}{l}3750 \\
3713 \\
3656 \\
4283\end{array}$ & $\begin{array}{r}190 \\
220 \\
95 \\
85\end{array}$ & $\begin{array}{l}3382 \\
3385 \\
3446 \\
4024\end{array}$ & $\begin{array}{l}10 \\
20 \\
15 \\
10\end{array}$ & $\begin{array}{l}3234 \\
3202 \\
3231 \\
3101 \\
3795\end{array}$ & $\begin{array}{r}610 \\
590 \\
415 \\
50 \\
330\end{array}$ & $\begin{array}{l}2160 \\
2372 \\
2447 \\
3006 \\
3075\end{array}$ & $\begin{array}{r}300 \\
160 \\
175 \\
0 \\
150\end{array}$ & $\begin{array}{l}1390 \\
1632 \\
1686 \\
2485 \\
2511\end{array}$ & $\begin{array}{l}150 \\
170 \\
150 \\
150 \\
150\end{array}$ & $\begin{array}{r}768 \\
926 \\
1001\end{array}$ & $\begin{array}{l}0 \\
0 \\
0\end{array}$ \\
\hline $\begin{array}{l}\text { O1N21E02BCCA } \\
\text { OIN21E23AC } \\
01 N 21 E 26 A A \\
01 N 22 E 03 C D \\
\text { OINZ3E 19DB }\end{array}$ & $\begin{array}{l}1-N O R D \\
1-23 H E \\
1 \text { KEATI } \\
1 \text { YELLL } \\
1-19\end{array}$ & $\begin{array}{r}19 \\
9 \\
10 \\
10\end{array}$ & $\begin{array}{l}3983 \\
4144 \\
4187 \\
3934 \\
4042\end{array}$ & & & & & & & & & & & & 3723 & 95 & $\begin{array}{l}3682 \\
3580\end{array}$ & $\begin{array}{r}290 \\
0\end{array}$ & $\begin{array}{l}3092 \\
3105\end{array}$ & $\begin{array}{l}125 \\
130\end{array}$ & 2222 & 0 \\
\hline $\begin{array}{l}01 N 24 E 06 A C \\
\text { OIN24E } 16 D A C A \\
\text { IINZBEO1BAC } \\
\text { OIN2BEOGAA } \\
\text { OIN29E1BDC }\end{array}$ & $\begin{array}{l}\text { 1MCFAR } \\
9-16 \\
1-\text { HERF } \\
\text { IWALTE } \\
\text { 1HEREF }\end{array}$ & $\begin{array}{r}9 \\
6 \\
9 \\
10 \\
10\end{array}$ & $\begin{array}{l}4227 \\
3692 \\
3564 \\
3112 \\
3590\end{array}$ & & & & & & & & & & & & & & & & & & $\begin{array}{l}2842 \\
2983\end{array}$ & $\begin{array}{l}0 \\
0\end{array}$ \\
\hline $\begin{array}{l}\text { OIN30E 08DCBD } \\
\text { OIN31E20CADB } \\
\text { IN INZE26BB } \\
\text { OIN34E25ABCA } \\
\text { OIN35E O4AD }\end{array}$ & $\begin{array}{l}1-V E R M \\
23-20 \\
11-26 \\
1-25 \\
1-8\end{array}$ & $\begin{array}{l}10 \\
10 \\
10 \\
10 \\
10\end{array}$ & $\begin{array}{l}3400 \\
3317 \\
3160 \\
3358 \\
3273\end{array}$ & & & & & & 3057 & 75 & 2821 & 255 & $\begin{array}{l}3160 \\
2473\end{array}$ & $\begin{array}{l}0 \\
0\end{array}$ & $\begin{array}{l}2170 \\
1553\end{array}$ & $\begin{array}{l}80 \\
90\end{array}$ & $\begin{array}{l}1889 \\
1314\end{array}$ & $\begin{array}{l}0 \\
0\end{array}$ & $\begin{array}{l}3100 \\
1595\end{array}$ & 45 & $\begin{array}{r}2314 \\
2823 \\
899\end{array}$ & $\begin{array}{l}0 \\
0\end{array}$ \\
\hline $\begin{array}{l}\text { OIN35E3ZCC } \\
01 N 37 E 12 C C C \\
\text { OIN37EZIAA } \\
\text { OIN4OEI3AA } \\
\text { OIN4IEZSDD }\end{array}$ & $\begin{array}{l}12-1 \\
\text { LUTHE } 1 \\
1=13 N P \\
l=N P\end{array}$ & $\begin{array}{r}10 \\
12 \\
8 \\
11 \\
12\end{array}$ & $\begin{array}{l}3378 \\
3546 \\
3286 \\
3448 \\
3093\end{array}$ & & & & & & $\begin{array}{l}2363 \\
2273\end{array}$ & $\begin{array}{r}85 \\
100\end{array}$ & $\begin{array}{l}2521 \\
2144 \\
2005\end{array}$ & $\begin{array}{l}185 \\
160 \\
220\end{array}$ & $\begin{array}{l}2052 \\
1670 \\
1545\end{array}$ & $\begin{array}{l}0 \\
0 \\
0\end{array}$ & $\begin{array}{r}1131 \\
829 \\
759\end{array}$ & $\begin{array}{l}80 \\
70 \\
65\end{array}$ & $\begin{array}{l}918 \\
665 \\
549\end{array}$ & $\begin{array}{l}0 \\
0 \\
5\end{array}$ & $\begin{array}{l}666 \\
299 \\
215\end{array}$ & $\begin{array}{l}25 \\
30 \\
30\end{array}$ & $\begin{array}{l}-197 \\
-703\end{array}$ & 0 \\
\hline 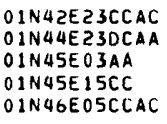 & $\begin{array}{l}2-N P \\
1-23 \\
1-H E A D \\
1 L I S C \\
1-5\end{array}$ & $\begin{array}{r}12 \\
04 \\
12 \\
10 \\
4\end{array}$ & $\begin{array}{l}3053 \\
2838 \\
2988 \\
3025 \\
3132\end{array}$ & & & & $\begin{array}{l}2515 \\
2722 \\
2645 \\
2721\end{array}$ & $\begin{array}{l}170 \\
200 \\
230\end{array}$ & $\begin{array}{l}2250 \\
2217 \\
2177 \\
2281\end{array}$ & $\begin{array}{r}130 \\
90 \\
75 \\
60\end{array}$ & $\begin{array}{l}1940 \\
1882 \\
1925 \\
1936\end{array}$ & $\begin{array}{l}160 \\
215 \\
180 \\
150\end{array}$ & $\begin{array}{l}1535 \\
1537 \\
1632 \\
1609\end{array}$ & $\begin{array}{l}0 \\
0 \\
0\end{array}$ & $\begin{array}{l}768 \\
785 \\
884 \\
866\end{array}$ & $\begin{array}{l}75 \\
50 \\
70\end{array}$ & $\begin{array}{l}526 \\
567 \\
675 \\
661\end{array}$ & $\begin{array}{l}0 \\
0 \\
0\end{array}$ & 220 & 45 & $\begin{array}{l}-711 \\
-625\end{array}$ & 0 \\
\hline
\end{tabular}




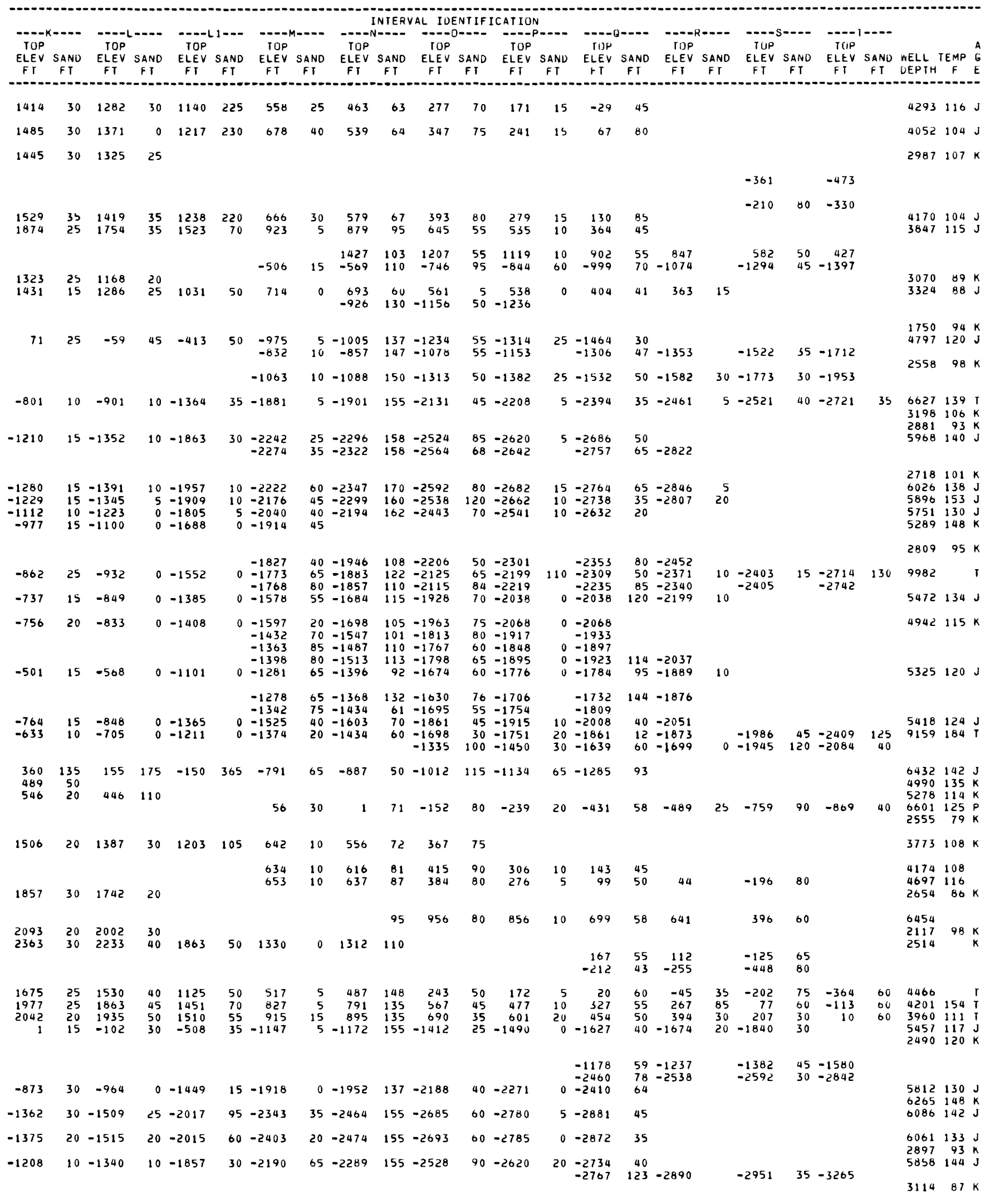


TABLE 2. -- SELECTED geOLUGIC DATA -- CONTINUED

\begin{tabular}{|c|c|c|c|c|c|c|c|c|c|c|c|c|c|c|c|c|c|c|c|c|c|c|}
\hline LOCAL NUMBEK & $\begin{array}{l}\text { WELL } \\
\text { NAME }\end{array}$ & $\begin{array}{l}K B \\
F I\end{array}$ & $\begin{array}{l}\text { LAND } \\
\text { ELEV } \\
\text { FT }\end{array}$ & $\begin{array}{l}--A-- \\
\text { SAND } \\
\text { FT }\end{array}$ & $\begin{array}{l}\text { TOP } \\
\text { ELEV } \\
\text { FT }\end{array}$ & $\begin{array}{l}\text { SAND } \\
\text { FT }\end{array}$ & $\begin{array}{c}-A-C \\
\text { ELEP } \\
F T\end{array}$ & $\begin{array}{l}\text { SAND } \\
\text { FT }\end{array}$ & $\begin{array}{l}\text { TOP } \\
\text { ELEV } \\
\text { FT }\end{array}$ & $\begin{array}{l}\text { SAND } \\
\text { FI }\end{array}$ & $\begin{array}{l}\text { INTERV } \\
\text { IOP } \\
\text { ELEV } \\
\text { FT }\end{array}$ & $\begin{array}{l}\text { IAL ID } \\
\text { SAND } \\
\text { FT }\end{array}$ & $\begin{array}{c}\text { ENTIF } \\
\text { ELUP } \\
\text { ET }\end{array}$ & $\begin{array}{c}\text { SAND } \\
\text { FT }\end{array}$ & $\begin{array}{c}\text { IOP } \\
\text { ELEV } \\
\text { FT }\end{array}$ & $\begin{array}{c}\text { SAND } \\
\text { FT }\end{array}$ & $\begin{array}{c}\text { IUP } \\
\text { ELEV } \\
\text { FT }\end{array}$ & $\begin{array}{c}\text { SAND } \\
\text { FT }\end{array}$ & $\begin{array}{l}\text { TUP } \\
\text { ELEV } \\
F T\end{array}$ & $\begin{array}{c}\text { SAND } \\
\text { FT }\end{array}$ & $\begin{array}{c}\text { ELEV } \\
\text { FT }\end{array}$ & $\begin{array}{l}\text { J-E-D } \\
\text { SAND } \\
\text { FT }\end{array}$ \\
\hline $\begin{array}{l}\text { OIN } \\
\text { OIN } 48 E \text { IIACBCAA } \\
\text { OIN49EOSBCDAC } \\
\text { OINSOEZIACD } \\
\text { OINSIEZTCCCA }\end{array}$ & $\begin{array}{l}1-11 B N \\
1-32 \\
1-A L L E \\
1 M C I N T \\
27-1\end{array}$ & $\begin{array}{r}8 \\
4 \\
12 \\
12 \\
11\end{array}$ & $\begin{array}{l}2964 \\
3182 \\
3003 \\
3267 \\
2976\end{array}$ & & & & $\begin{array}{l}2709 \\
2728\end{array}$ & $\begin{array}{l}170 \\
160\end{array}$ & $\begin{array}{l}2342 \\
2348 \\
2610 \\
2790\end{array}$ & $\begin{array}{r}135 \\
140 \\
95 \\
110\end{array}$ & $\begin{array}{l}2037 \\
1921 \\
2265 \\
2413\end{array}$ & $\begin{array}{l}235 \\
130 \\
230 \\
245\end{array}$ & $\begin{array}{l}1637 \\
1036 \\
1825 \\
2013\end{array}$ & $\begin{array}{l}0 \\
0 \\
0 \\
0\end{array}$ & $\begin{array}{r}895 \\
907 \\
1091 \\
1277\end{array}$ & $\begin{array}{l}60 \\
65 \\
60 \\
21\end{array}$ & $\begin{array}{r}724 \\
742 \\
931 \\
1121\end{array}$ & $\begin{array}{l}0 \\
0 \\
0 \\
0\end{array}$ & $\begin{array}{l}606 \\
792\end{array}$ & $\begin{array}{l}10 \\
10\end{array}$ & $\begin{array}{l}-370 \\
-178\end{array}$ & $\begin{array}{l}0 \\
0\end{array}$ \\
\hline $\begin{array}{l}\text { OINS2E25AA } \\
\text { OINS3E26CBCA } \\
\text { OINS4E24BB } \\
\text { OIN55E27CD } \\
\text { OIN57E33DDCA }\end{array}$ & $\begin{array}{l}25-1 \\
S-26-1 \\
1 \text { BURD } \\
1-27 \\
33-16\end{array}$ & $\begin{array}{r}8 \\
7 \\
11 \\
5 \\
11\end{array}$ & $\begin{array}{l}3298 \\
2900 \\
2922 \\
2908 \\
3628\end{array}$ & & & & & & 2846 & 75 & $\begin{array}{l}2513 \\
2691 \\
3493\end{array}$ & $\begin{array}{l}150 \\
135 \\
230\end{array}$ & $\begin{array}{l}2191 \\
2319 \\
2514 \\
2623 \\
3124\end{array}$ & $\begin{array}{l}0 \\
0 \\
0 \\
0 \\
0\end{array}$ & $\begin{array}{l}1465 \\
1597 \\
1778 \\
1933 \\
2362\end{array}$ & $\begin{array}{r}10 \\
0 \\
0 \\
0 \\
0\end{array}$ & $\begin{array}{l}1319 \\
1450 \\
1628 \\
1770 \\
2199\end{array}$ & $\begin{array}{l}0 \\
0 \\
0 \\
0 \\
0\end{array}$ & $\begin{array}{r}990 \\
1108 \\
1267 \\
1403 \\
1820\end{array}$ & $\begin{array}{r}10 \\
10 \\
10 \\
0 \\
0\end{array}$ & $\begin{array}{r}-1 \\
71 \\
245 \\
382 \\
803\end{array}$ & $\begin{array}{l}0 \\
0 \\
0 \\
0 \\
0\end{array}$ \\
\hline $\begin{array}{l}\text { OINSBE 28DCDB } \\
\text { OINGOE19DCA } \\
\text { OINGIEO3ACDB } \\
\text { OIS11E25BD } \\
\text { OISIGEOZCC }\end{array}$ & $\begin{array}{l}D-108 \\
1-P H E L \\
32-3 \\
22-25 \\
1 \text { HOEFL }\end{array}$ & $\begin{array}{l}11 \\
13 \\
13 \\
11 \\
11\end{array}$ & $\begin{array}{l}4090 \\
3205 \\
3071 \\
4686 \\
4433\end{array}$ & 220 & & & 3779 & 125 & 3591 & 95 & 3366 & 150 & $\begin{array}{l}2983 \\
2615\end{array}$ & $\begin{array}{l}0 \\
0\end{array}$ & $\begin{array}{l}2183 \\
1768 \\
1539\end{array}$ & $\begin{array}{l}0 \\
0 \\
0\end{array}$ & $\begin{array}{l}2047 \\
1648 \\
1439\end{array}$ & $\begin{array}{l}0 \\
0 \\
0\end{array}$ & $\begin{array}{l}1657 \\
1298 \\
1124\end{array}$ & $\begin{array}{l}0 \\
5 \\
0\end{array}$ & $\begin{array}{r}650 \\
304 \\
97 \\
1582\end{array}$ & $\begin{array}{l}0 \\
0 \\
5 \\
0\end{array}$ \\
\hline $\begin{array}{l}01517 E 22 D A C \\
01 S 17 E 25 B B \\
01 S 1 B E 01 C D B \\
01 S 18 E 12 B C \\
01 S 19 E 02 B C A\end{array}$ & $\begin{array}{l}1-22 \\
1-25 \\
100 S T \\
12-12 \\
1 \text { BOKM }\end{array}$ & $\begin{array}{r}11 \\
10 \\
9 \\
11 \\
9\end{array}$ & $\begin{array}{l}4175 \\
3836 \\
4116 \\
4060 \\
4522\end{array}$ & & & & & & & & 3516 & 205 & $\begin{array}{l}3111 \\
3885 \\
4132\end{array}$ & $\begin{array}{l}55 \\
80 \\
40\end{array}$ & $\begin{array}{l}2732 \\
3630 \\
3951\end{array}$ & $\begin{array}{l}310 \\
250 \\
325\end{array}$ & $\begin{array}{l}1892 \\
2894 \\
3150\end{array}$ & $\begin{array}{l}125 \\
185 \\
120\end{array}$ & 2791 & 200 & 1881 & 0 \\
\hline $\begin{array}{l}01 S 20 E 34 A A C \\
01 S 21 E 16 A A C C \\
01 S 22 E 15 C D A C \\
01 S 23 E 25 D \\
01 S 23 E 34 C B\end{array}$ & $\begin{array}{l}1 \text { NELS } \\
1-16 \\
1 \text { LACK } \\
10-25 \\
1 \text { MAGEL }\end{array}$ & $\begin{array}{r}9 \\
10 \\
9 \\
10 \\
10\end{array}$ & $\begin{array}{l}4398 \\
4194 \\
3798 \\
4027 \\
3550\end{array}$ & & & & & & & & & & $\begin{array}{l}4214 \\
3941\end{array}$ & $\begin{array}{l}20 \\
60\end{array}$ & $\begin{array}{l}4097 \\
3492\end{array}$ & $\begin{array}{l}300 \\
215\end{array}$ & $\begin{array}{l}3364 \\
3018 \\
3475\end{array}$ & $\begin{array}{l}175 \\
140 \\
140\end{array}$ & $\begin{array}{l}3002 \\
2719 \\
3257\end{array}$ & $\begin{array}{l}195 \\
165 \\
150\end{array}$ & $\begin{array}{l}2172 \\
2009 \\
2454 \\
3332\end{array}$ & $\begin{array}{l}0 \\
0 \\
0\end{array}$ \\
\hline $\begin{array}{l}01 S 24 E 29 B B \\
01 S 31 E 35 A A C \\
01 S 32 E 23 B D \\
01 S 32 E 26 B 8 D \\
01 S 33 E 19 B B D\end{array}$ & $\begin{array}{l}4-29 \\
1-35 \\
1 \text { SNYDE } \\
1 \text { GUST } \\
4-19\end{array}$ & $\begin{array}{l}6 \\
5 \\
9 \\
4 \\
5\end{array}$ & $\begin{array}{l}3588 \\
3232 \\
3027 \\
3124 \\
3073\end{array}$ & & & & & & & & & & & & & & & & & & 3404 & 0 \\
\hline 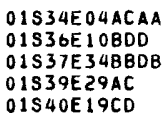 & $\begin{array}{l}1 \text { OTTU } \\
T 44-10 \\
4-34 \\
N P 1 \\
N P 1\end{array}$ & $\begin{array}{l}9 \\
11 \\
11\end{array}$ & $\begin{array}{l}3193 \\
3433 \\
3450 \\
2885 \\
3626\end{array}$ & & & & & & & & 2756 & 110 & $\begin{array}{l}2420 \\
1793 \\
1666\end{array}$ & $\begin{array}{l}0 \\
0\end{array}$ & $\begin{array}{r}1506 \\
989 \\
839\end{array}$ & $\begin{array}{l}70 \\
75 \\
70\end{array}$ & $\begin{array}{r}1371 \\
750 \\
671\end{array}$ & $\begin{array}{l}0 \\
0 \\
0\end{array}$ & $\begin{array}{r}1015 \\
453 \\
276\end{array}$ & 25 & $\begin{array}{r}3086 \\
296\end{array}$ & $\begin{array}{l}0 \\
0\end{array}$ \\
\hline $\begin{array}{l}01 S 42 E 03 B B \\
01 S 42 E 15 D C D B \\
01 S 43 E 01 C C \\
01 S 44 E 15 B B D B \\
01 S 45 E 23 C C A C\end{array}$ & $\begin{array}{l}11-3 \\
1 \text { GREE } \\
\text { NP } 3 \\
11-15 \\
6 \text { NPRR }\end{array}$ & $\begin{array}{l}11 \\
10 \\
11 \\
6 \\
10\end{array}$ & $\begin{array}{l}2994 \\
3251 \\
3155 \\
2818 \\
3199\end{array}$ & $\begin{array}{l}315 \\
320\end{array}$ & $\begin{array}{l}2693 \\
2663 \\
2901\end{array}$ & $\begin{array}{r}35 \\
80 \\
145\end{array}$ & $\begin{array}{l}2494 \\
2396 \\
2529 \\
2632\end{array}$ & $\begin{array}{l}205 \\
240 \\
130 \\
220\end{array}$ & $\begin{array}{l}2141 \\
2116 \\
2276 \\
2344\end{array}$ & $\begin{array}{r}55 \\
90 \\
105 \\
65\end{array}$ & $\begin{array}{l}1941 \\
1856 \\
1984 \\
2179\end{array}$ & $\begin{array}{l}230 \\
170 \\
165 \\
260\end{array}$ & $\begin{array}{l}1471 \\
1436 \\
1489 \\
1459\end{array}$ & $\begin{array}{l}0 \\
0 \\
0 \\
0\end{array}$ & $\begin{array}{l}676 \\
701 \\
722 \\
715\end{array}$ & $\begin{array}{l}60 \\
60 \\
60 \\
55\end{array}$ & $\begin{array}{l}481 \\
476 \\
519 \\
504\end{array}$ & $\begin{array}{r}10 \\
0 \\
0 \\
0\end{array}$ & $\begin{array}{l}155 \\
206 \\
239\end{array}$ & $\begin{array}{l}25 \\
40 \\
40\end{array}$ & $\begin{array}{l}-785 \\
-794 \\
-792\end{array}$ & $\begin{array}{l}0 \\
0\end{array}$ \\
\hline $\begin{array}{l}01 S 46 E 35 B B A C \\
01 S 47 E 35 C C \\
01 S 4 B E 02 D C C A \\
01 S 49 E 090 D \\
01 S 50 E 19 C \triangle D A\end{array}$ & $\begin{array}{l}1 \text { CHOA } \\
35-10 \\
1 \text { HOLM } \\
12 \text { NPR } \\
1 \text { JANS }\end{array}$ & $\begin{array}{r}7 \\
10 \\
10 \\
10 \\
5\end{array}$ & $\begin{array}{l}3352 \\
3446 \\
3110 \\
3172 \\
3282\end{array}$ & $\begin{array}{l}145 \\
235\end{array}$ & $\begin{array}{l}3043 \\
3111\end{array}$ & $\begin{array}{l}155 \\
160\end{array}$ & $\begin{array}{l}2554 \\
2716 \\
2795 \\
2820 \\
2987\end{array}$ & $\begin{array}{l}180 \\
200 \\
160 \\
110 \\
105\end{array}$ & $\begin{array}{l}2128 \\
2301 \\
2381 \\
2447 \\
2625\end{array}$ & $\begin{array}{r}80 \\
80 \\
120 \\
50 \\
120\end{array}$ & $\begin{array}{l}1883 \\
1841 \\
2036 \\
2082 \\
2167\end{array}$ & $\begin{array}{l}220 \\
150 \\
160 \\
145 \\
175\end{array}$ & $\begin{array}{l}1441 \\
1467 \\
1650 \\
1784 \\
1795\end{array}$ & $\begin{array}{l}0 \\
0 \\
0 \\
0 \\
0\end{array}$ & $\begin{array}{r}709 \\
751 \\
925 \\
1041 \\
1044\end{array}$ & $\begin{array}{l}50 \\
50 \\
60 \\
35 \\
20\end{array}$ & $\begin{array}{l}509 \\
574 \\
752 \\
874 \\
895\end{array}$ & $\begin{array}{l}0 \\
0 \\
0 \\
0 \\
0\end{array}$ & $\begin{array}{l}237 \\
307 \\
482 \\
592\end{array}$ & $\begin{array}{r}25 \\
10 \\
10 \\
5\end{array}$ & $\begin{array}{l}-774 \\
-764 \\
-576 \\
-398\end{array}$ & $\begin{array}{l}0 \\
0 \\
0 \\
0\end{array}$ \\
\hline $\begin{array}{l}01 S 51 E 13 C C D B \\
01 S 52 E 13 A A B D \\
01 S 52 E 21 B B B D \\
01 S 52 E 27 D C \\
01 S 52 E 31 C C\end{array}$ & $\begin{array}{l}13-1 \\
41-13 \\
1 \text { NPRR } \\
1-N P R R \\
5-31-2\end{array}$ & $\begin{array}{r}9 \\
10 \\
10 \\
10 \\
7\end{array}$ & $\begin{array}{l}3086 \\
3266 \\
3117 \\
3213 \\
3227\end{array}$ & & & & & 150 & $\begin{array}{l}2685 \\
2816\end{array}$ & $\begin{array}{l}80 \\
75\end{array}$ & $\begin{array}{l}2480 \\
2496\end{array}$ & $\begin{array}{l}180 \\
170\end{array}$ & $\begin{array}{l}2045 \\
2171\end{array}$ & $\begin{array}{l}0 \\
0\end{array}$ & $\begin{array}{l}1300 \\
1421\end{array}$ & $\begin{array}{r}20 \\
0\end{array}$ & $\begin{array}{l}1157 \\
1271\end{array}$ & $\begin{array}{l}0 \\
0\end{array}$ & $\begin{array}{l}836 \\
946\end{array}$ & $\begin{array}{l}5 \\
5\end{array}$ & $\begin{array}{r}-45 \\
11\end{array}$ & $\begin{array}{l}0 \\
0\end{array}$ \\
\hline $\begin{array}{l}01 S 53 E 15 D 0 \\
01353 E 35 C C \\
01 S 55 E 15 B C A C \\
01 S 55 E 17 A A A C \\
01 S 55 E 26 C A\end{array}$ & $\begin{array}{l}\text { NPRR } 1 \\
1-A N P \\
D-126 \\
1=17 B L \\
1-26\end{array}$ & $\begin{array}{l}11 \\
7 \\
10 \\
10 \\
10\end{array}$ & $\begin{array}{l}2924 \\
2928 \\
3093 \\
2976 \\
3046\end{array}$ & & & & & & & & 2680 & 190 & $\begin{array}{l}2278 \\
2713\end{array}$ & $\begin{array}{l}0 \\
0\end{array}$ & $\begin{array}{l}1551 \\
2012\end{array}$ & 0 & $\begin{array}{l}1405 \\
1858\end{array}$ & 0 & $\begin{array}{l}1064 \\
1498\end{array}$ & 5 & $\begin{array}{l}114 \\
530\end{array}$ & 0 \\
\hline $\begin{array}{l}01 S 56 E: 700 \\
01 S 56 E 350 A \\
01 S 57 E 210 D D B \\
01 S 60 E 20 D B \\
01 S 62 E 06\end{array}$ & $\begin{array}{l}1-17 \\
1 \text { PURE } \\
\text { D-1 06 } \\
\text { ICURRY } \\
\text { GOVT } 1\end{array}$ & $\begin{array}{l}7 \\
13 \\
10 \\
14 \\
13\end{array}$ & $\begin{array}{l}2915 \\
3413 \\
3733 \\
3140 \\
3353\end{array}$ & & & & & & & & & & $\begin{array}{l}2672 \\
3223\end{array}$ & $\begin{array}{l}0 \\
0\end{array}$ & $\begin{array}{l}2000 \\
2490\end{array}$ & 0 & $\begin{array}{l}1835 \\
2315\end{array}$ & 0 & $\begin{array}{l}1477 \\
1948\end{array}$ & 0 & $\begin{array}{l}476 \\
979\end{array}$ & 0 \\
\hline $\begin{array}{l}01 S 62 E 07 B C \\
02 S 11 E 27 D A \\
02 S 12 E 01 A A A C \\
02 S 16 E 20 A B \\
02 S 17 E 22 A A\end{array}$ & $\begin{array}{l}1 \text { GRENZ } \\
1 \text { LARK } \\
1 \text {-BN } \\
1-\text { MOIH } \\
41-22\end{array}$ & $\begin{array}{l}9 \\
10 \\
10 \\
12 \\
10\end{array}$ & $\begin{array}{l}3276 \\
6276 \\
4929 \\
4384 \\
4726\end{array}$ & & & & & & 3015 & 50 & 2653 & 270 & 2265 & 0 & $\begin{array}{l}1515 \\
3491\end{array}$ & 330 & $\begin{array}{l}1406 \\
2928\end{array}$ & 160 & $\begin{array}{l}1025 \\
2476\end{array}$ & $\begin{array}{r}5 \\
150\end{array}$ & $\begin{array}{r}29 \\
5410 \\
1831\end{array}$ & $\begin{array}{l}0 \\
0\end{array}$ \\
\hline $\begin{array}{l}02 S 19 E 27 A A C B \\
02 S 19 E 33 A D D \\
02 S 21 E 11 D C A C \\
02 S 21 E 19 C D \\
02 S 23 E 07 A D B D\end{array}$ & $\begin{array}{l}1-27 \\
\text { GOVI } 1 \\
1-P E T E \\
1 \text { MURAN } \\
1-\text { WAGN }\end{array}$ & $\begin{array}{r}10 \\
11 \\
9 \\
11 \\
7\end{array}$ & $\begin{array}{l}3983 \\
4373 \\
4222 \\
3880 \\
3808\end{array}$ & & & & & & 3705 & 115 & 3413 & 160 & 3068 & 60 & $\begin{array}{l}2803 \\
3724\end{array}$ & $\begin{array}{l}275 \\
310\end{array}$ & $\begin{array}{l}2255 \\
3221\end{array}$ & $\begin{array}{l}240 \\
248\end{array}$ & 2973 & 140 & $\begin{array}{l}2136 \\
2997\end{array}$ & 0 \\
\hline $\begin{array}{l}02 S 24 E 26 B B D \\
02 S 26 E 11 B B D \\
02 S 35 E 17 B A D C \\
02 S 35 E 19 B B A C \\
02 S 35 E 27 C C\end{array}$ & $\begin{array}{l}1-L A I R \\
4-11 \\
1 \text { CROW } \\
1 \text { ORCH } \\
1 \text { CROW }\end{array}$ & $\begin{array}{r}5 \\
5 \\
9 \\
9 \\
10\end{array}$ & $\begin{array}{l}3336 \\
3618 \\
3206 \\
3125 \\
3333\end{array}$ & & & & & & & & & & & & & & 2781 & 0 & 2383 & 25 & 1633 & 0 \\
\hline
\end{tabular}




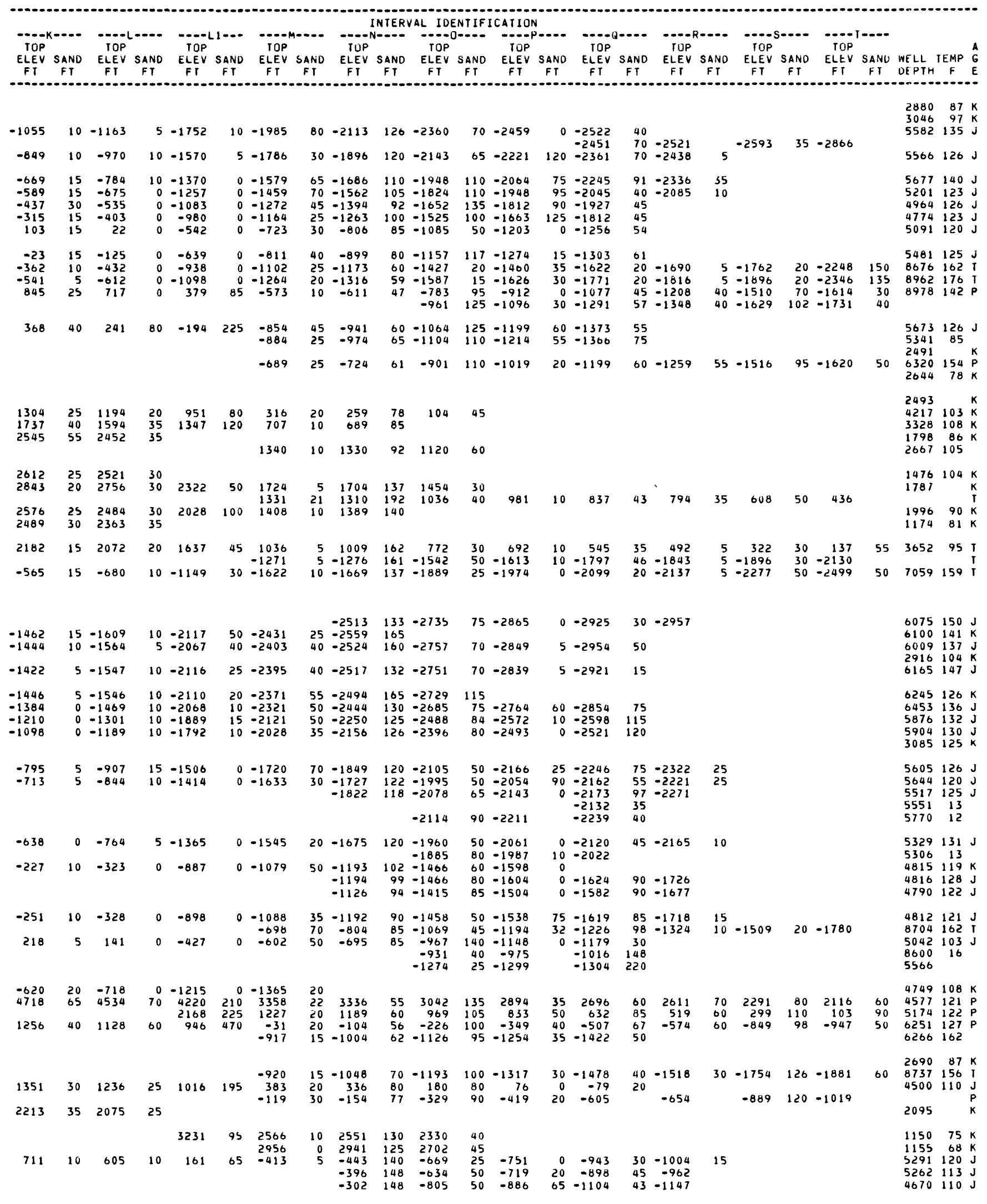


TABLE 2. -- SELECTED GEOLOGIC DATA. - CONTINUEO

\begin{tabular}{|c|c|c|c|c|c|c|c|c|c|c|c|c|c|c|c|c|c|c|c|c|c|c|}
\hline LOCAL NUMBER & $\begin{array}{l}\text { WELL } \\
\text { NAME }\end{array}$ & $\begin{array}{l}K B \\
F T\end{array}$ & $\begin{array}{l}\text { LAND } \\
\text { ELEV } \\
\text { FI }\end{array}$ & $\begin{array}{l}\text { SAND } \\
\text { FT }\end{array}$ & $\begin{array}{l}\text { TOP } \\
\text { ELEV } \\
\text { FT }\end{array}$ & $\begin{array}{l}\text { SAND } \\
\text { FT }\end{array}$ & $\begin{array}{l}-O-C \text { TOP } \\
\text { ELEV } \\
\text { FT }\end{array}$ & $\begin{array}{l}\text { SAND } \\
\text { FT }\end{array}$ & $\begin{array}{c}\text { IOP } \\
\text { ELEV } \\
F T\end{array}$ & $\begin{array}{l}\text { SAND } \\
\text { FI }\end{array}$ & $\begin{array}{l}\text { TOP } \\
\text { ELEV }\end{array}$ & $\begin{array}{l}\text { SAND } \\
\text { FT }\end{array}$ & $\begin{array}{c}- \\
\text { TOP } \\
\text { ELEV } \\
\text { FT }\end{array}$ & SAMD & $\begin{array}{c}\text { ELEV } \\
\text { TOP }\end{array}$ & $\begin{array}{l}\text { SANO } \\
\text { FY }\end{array}$ & $\begin{array}{l}\text { ELEV } \\
\text { FT }\end{array}$ & 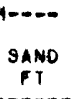 & $\begin{array}{l}\text { TOP } \\
\text { ELEV } \\
F T\end{array}$ & 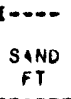 & $\begin{array}{l}\text { TOP } \\
\text { ELEV }\end{array}$ & SAND \\
\hline $\begin{array}{l}02 S 40 E 19 B D B C \\
02 S 42 E 03 A A \\
02 S 44 E 32 A A A C \\
02 S 46 E 33 B A A C \\
02 S 4719 C C\end{array}$ & $\begin{array}{l}22-19 \\
4-N P \\
C F 1-32 \\
21=33 \\
1 N P\end{array}$ & $\begin{array}{r}11 \\
12 \\
8 \\
9 \\
12\end{array}$ & $\begin{array}{l}3546 \\
3487 \\
3290 \\
3468 \\
4057\end{array}$ & $\begin{array}{l}655 \\
460\end{array}$ & $\begin{array}{l}2645 \\
2837\end{array}$ & $\begin{array}{r}50 \\
130\end{array}$ & $\begin{array}{l}2797 \\
2455 \\
2512\end{array}$ & $\begin{array}{l}240 \\
180 \\
230\end{array}$ & $\begin{array}{l}2397 \\
2164 \\
2072\end{array}$ & $\begin{array}{r}100 \\
75 \\
90\end{array}$ & $\begin{array}{l}2107 \\
1929 \\
1827\end{array}$ & $\begin{array}{l}300 \\
180 \\
240\end{array}$ & $\begin{array}{l}1692 \\
1441 \\
1205 \\
1302\end{array}$ & $\begin{array}{l}0 \\
0 \\
0 \\
0\end{array}$ & $\begin{array}{l}917 \\
661 \\
502 \\
575\end{array}$ & $\begin{array}{l}75 \\
60 \\
35 \\
55\end{array}$ & $\begin{array}{l}727 \\
361 \\
305 \\
397\end{array}$ & $\begin{array}{l}0 \\
0 \\
5 \\
0\end{array}$ & $\begin{array}{r}387 \\
147 \\
44\end{array}$ & $\begin{array}{l}25 \\
30 \\
50\end{array}$ & $\begin{array}{r}-478 \\
-795 \\
-1017\end{array}$ & $\begin{array}{l}0 \\
0 \\
0\end{array}$ \\
\hline $\begin{array}{l}\text { O2S47E23AACA } \\
02 S 48 E 26 D A A \\
02 S 49 E 21 C D \\
02 S 50 \text { 11 } \\
02 A B D \\
02 S 50 E 15 D D\end{array}$ & $\begin{array}{l}11-N P \\
1 \text { GASK } \\
13-N P R \\
41-11 \\
S-15-1\end{array}$ & $\begin{array}{l}10 \\
11 \\
10 \\
10 \\
10\end{array}$ & $\begin{array}{l}3633 \\
3360 \\
3535 \\
3266 \\
3261\end{array}$ & $\begin{array}{l}455 \\
195 \\
265\end{array}$ & $\begin{array}{l}2853 \\
3046 \\
3085\end{array}$ & $\begin{array}{l}205 \\
140 \\
160\end{array}$ & $\begin{array}{l}2458 \\
2728 \\
2685\end{array}$ & $\begin{array}{l}255 \\
160 \\
100\end{array}$ & $\begin{array}{l}2148 \\
2386 \\
2335\end{array}$ & $\begin{array}{r}110 \\
80 \\
80\end{array}$ & $\begin{array}{l}1873 \\
1976 \\
2025\end{array}$ & $\begin{array}{l}310 \\
210 \\
170\end{array}$ & $\begin{array}{l}1439 \\
1481 \\
1620\end{array}$ & $\begin{array}{l}0 \\
0 \\
0\end{array}$ & $\begin{array}{l}723 \\
771 \\
900\end{array}$ & $\begin{array}{r}30 \\
20 \\
0\end{array}$ & $\begin{array}{l}553 \\
626 \\
760\end{array}$ & $\begin{array}{l}0 \\
0 \\
0\end{array}$ & $\begin{array}{l}290 \\
365 \\
490\end{array}$ & $\begin{array}{l}30 \\
40 \\
20\end{array}$ & $\begin{array}{l}-759 \\
-676 \\
-532\end{array}$ & $\begin{array}{l}0 \\
0 \\
0\end{array}$ \\
\hline $\begin{array}{l}02550 E 23 A A \\
02550 E 268 C \\
02 S 51 E 21 C C \\
02 S 52 E 130 C \\
02 S 52 E 298 B\end{array}$ & $\begin{array}{l}23-1 N P \\
1 E M M O N \\
21-1 N P \\
13-2 N P \\
29-3 N P\end{array}$ & $\begin{array}{r}8 \\
10 \\
8 \\
8 \\
10\end{array}$ & $\begin{array}{l}3248 \\
3277 \\
3130 \\
2877 \\
3221\end{array}$ & & 3066 & 155 & 2896 & $\begin{array}{r}244 \\
60\end{array}$ & $\begin{array}{l}2485 \\
2668 \\
2937\end{array}$ & $\begin{array}{r}83 \\
110 \\
85\end{array}$ & $\begin{array}{l}2331 \\
2368 \\
2491\end{array}$ & $\begin{array}{l}310 \\
160 \\
175\end{array}$ & $\begin{array}{l}1917 \\
1992 \\
2106\end{array}$ & $\begin{array}{l}0 \\
0 \\
0\end{array}$ & $\begin{array}{l}1170 \\
1228 \\
1361\end{array}$ & $\begin{array}{l}0 \\
0 \\
0\end{array}$ & $\begin{array}{l}1029 \\
1088 \\
1221\end{array}$ & $\begin{array}{l}0 \\
0 \\
0\end{array}$ & $\begin{array}{l}729 \\
782 \\
906\end{array}$ & $\begin{array}{r}10 \\
5\end{array}$ & $\begin{array}{l}-164 \\
-113 \\
-24\end{array}$ & $\begin{array}{l}0 \\
0 \\
0\end{array}$ \\
\hline $\begin{array}{l}02 S 53 E 13 B B \\
02 S 53 E 28 C B \\
02 S 54 E 09 A A D B \\
02 S 55 E 21 D D \\
02 S 57 E 23 C C\end{array}$ & $\begin{array}{l}D-118 \\
D-117 \\
D-133 \\
21=168 \\
1=23\end{array}$ & $\begin{array}{l}10 \\
10 \\
10 \\
10 \\
9\end{array}$ & $\begin{array}{l}2959 \\
3155 \\
2932 \\
3117 \\
3417\end{array}$ & & & & & & & & 2729 & 210 & $\begin{array}{l}2389 \\
2526 \\
2877\end{array}$ & $\begin{array}{l}0 \\
0 \\
0\end{array}$ & $\begin{array}{l}1643 \\
1787 \\
2177 \\
3010\end{array}$ & $\begin{array}{l}0 \\
0 \\
0 \\
0\end{array}$ & $\begin{array}{l}1495 \\
1637 \\
2017 \\
2816\end{array}$ & $\begin{array}{l}0 \\
0 \\
0 \\
0\end{array}$ & $\begin{array}{l}1164 \\
1289 \\
1669 \\
2471\end{array}$ & $\begin{array}{l}5 \\
0 \\
0 \\
0\end{array}$ & $\begin{array}{r}189 \\
304 \\
697 \\
1531\end{array}$ & $\begin{array}{l}0 \\
0 \\
0 \\
0\end{array}$ \\
\hline $\begin{array}{l}02 S 58 E 14 C C \\
02 S 58 E 17 C C A C \\
02 S 5 B E 29 D D \\
02 S 59 E 29 D B \\
02 S 60 E 21 B D\end{array}$ & $\begin{array}{l}1 \text { JOHN } \\
D=123 \\
D=104 \\
D=130 \\
21=6\end{array}$ & $\begin{array}{l}0 \\
10 \\
10 \\
10 \\
10\end{array}$ & $\begin{array}{l}3289 \\
3466 \\
3416 \\
3282 \\
3309\end{array}$ & & & & & & & & & & & & $\begin{array}{l}2904 \\
2921 \\
2544\end{array}$ & $\begin{array}{l}0 \\
0\end{array}$ & $\begin{array}{l}2722 \\
2742 \\
2374\end{array}$ & $\begin{array}{l}0 \\
0\end{array}$ & $\begin{array}{l}2354 \\
2372 \\
2007\end{array}$ & $\begin{array}{l}0 \\
0\end{array}$ & $\begin{array}{l}1449 \\
1494 \\
1093\end{array}$ & $\begin{array}{l}0 \\
0\end{array}$ \\
\hline $\begin{array}{l}02 S 61 E 20 B B C D \\
02 S 62 E 05 D C A C \\
03 S 12 E 05 D C B D \\
03 S 16 E 290 B C B \\
03 S 17 E 13 A C \\
03517\end{array}$ & $\begin{array}{l}D-111 \\
1-T U R B \\
\text { NPRR } 1 \\
90-278 \\
32-13\end{array}$ & $\begin{array}{r}10 \\
9 \\
10 \\
10 \\
11\end{array}$ & $\begin{array}{l}3560 \\
3262 \\
5750 \\
5408 \\
4548\end{array}$ & & & & & & & & $\begin{array}{l}3343 \\
2826\end{array}$ & $\begin{array}{r}75 \\
130\end{array}$ & $\begin{array}{l}3030 \\
2464\end{array}$ & $\begin{array}{l}0 \\
0\end{array}$ & $\begin{array}{l}2302 \\
1651\end{array}$ & $\begin{array}{l}0 \\
0\end{array}$ & $\begin{array}{l}2177 \\
1527\end{array}$ & $\begin{array}{l}0 \\
0\end{array}$ & $\begin{array}{l}1825 \\
1221\end{array}$ & $\begin{array}{r}10 \\
0\end{array}$ & $\begin{array}{l}885 \\
306\end{array}$ & $\begin{array}{l}0 \\
0\end{array}$ \\
\hline $\begin{array}{l}03 S 21 E O B C A A C \\
03 S 21 E 32 A C \\
03 S 22 E 21 C A \\
03 S 23 E 29 B C D B \\
03 S 24 E 26 C A\end{array}$ & $\begin{array}{l}1 \text { THOM } \\
1 \text { LENNI } \\
1 \text { GOLD } \\
\text { PAIN } 1 \\
\text { MOSSE }\end{array}$ & $\begin{array}{r}10 \\
10 \\
10 \\
6 \\
11\end{array}$ & $\begin{array}{l}3800 \\
4464 \\
4007 \\
3906 \\
4294\end{array}$ & & & & & & & & & & & & 3260 & 320 & $\begin{array}{l}2793 \\
3752\end{array}$ & $\begin{array}{l}195 \\
140\end{array}$ & $\begin{array}{l}2475 \\
3413\end{array}$ & $\begin{array}{l}150 \\
140\end{array}$ & $\begin{array}{l}1607 \\
2584 \\
3559\end{array}$ & $\begin{array}{l}0 \\
0 \\
0\end{array}$ \\
\hline $\begin{array}{l}\text { O3S24E26DDAA } \\
03525 E 14 D C \\
03530 E 30 A B \\
03531 E 21 A C \\
03531 E 21 A C B D\end{array}$ & $\begin{array}{l}4 \text { SPAE } \\
\text { : CLAN } \\
1 \text { CROW } \\
1 \text { CROW } \\
1 \text { CROW }\end{array}$ & $\begin{array}{r}2 \\
2 \\
11 \\
5 \\
5\end{array}$ & $\begin{array}{l}4334 \\
4514 \\
3463 \\
3242 \\
3242\end{array}$ & & & & & & & & & & & & & & & & & & & \\
\hline $\begin{array}{l}03535 E \text { OZDDBD } \\
03 S 35 E 1 \text { OBBDB } \\
03535 E 30 C A\end{array}$ & $\begin{array}{ll}1 & \text { CROW } \\
1 & \text { CROW } \\
1 & \text { BIG }\end{array}$ & $\begin{array}{r}0 \\
10 \\
10\end{array}$ & $\begin{array}{l}3383 \\
3244 \\
3112\end{array}$ & & & & & & & & & & & & 2904 & 110 & 2709 & 5 & 2324 & 50 & 1514 & 0 \\
\hline $\begin{array}{l}03537 E 03 B C \\
03543 E 02 A A\end{array}$ & $\begin{array}{l}1 \text { CRUW } \\
1-2\end{array}$ & 11 & $\begin{array}{l}3629 \\
3350\end{array}$ & 555 & 2521 & 125 & 2230 & 235 & 1894 & 115 & 1531 & 190 & $\begin{array}{l}2448 \\
1111\end{array}$ & 0 & $\begin{array}{r}1568 \\
386\end{array}$ & $\begin{array}{l}70 \\
40\end{array}$ & $\begin{array}{r}1434 \\
231\end{array}$ & 20 & $\begin{array}{l}998 \\
-68\end{array}$ & 50 & -1105 & 0 \\
\hline $\begin{array}{l}03 S 45 E 07 C C \\
03 S 46 E 18 D B C A \\
03 S 48 E 13 A A D A \\
03 S 48 E 34 B B \\
03 S 49 E 15 B B\end{array}$ & $\begin{array}{l}\text { NP-7 } \\
1 \text { SUPE } \\
1 \text { LNPRR } \\
1 \text { IRW IN } \\
1 \text { SNPRR }\end{array}$ & $\begin{array}{l}11 \\
14 \\
10 \\
11 \\
10\end{array}$ & $\begin{array}{l}3125 \\
3209 \\
3400 \\
3467 \\
3626\end{array}$ & 485 & 2973 & 120 & $\begin{array}{l}2295 \\
2518 \\
2480 \\
2606\end{array}$ & $\begin{array}{l}150 \\
240 \\
110 \\
160\end{array}$ & $\begin{array}{l}1956 \\
2173 \\
2260 \\
2266\end{array}$ & $\begin{array}{l}130 \\
190 \\
100 \\
95\end{array}$ & $\begin{array}{l}1556 \\
1693 \\
1883 \\
1956\end{array}$ & $\begin{array}{l}200 \\
235 \\
210 \\
190\end{array}$ & $\begin{array}{l}1166 \\
1210 \\
1515 \\
1605\end{array}$ & $\begin{array}{l}0 \\
5 \\
0\end{array}$ & $\begin{array}{l}465 \\
473 \\
797 \\
855\end{array}$ & $\begin{array}{r}45 \\
20 \\
0\end{array}$ & $\begin{array}{l}276 \\
338 \\
660 \\
734\end{array}$ & $\begin{array}{r}0 \\
10 \\
0 \\
0\end{array}$ & $\begin{array}{r}14 \\
78 \\
406 \\
466\end{array}$ & $\begin{array}{l}50 \\
45 \\
30 \\
30\end{array}$ & $\begin{array}{l}-999 \\
-955 \\
-705 \\
-644\end{array}$ & $\begin{array}{l}0 \\
0 \\
0\end{array}$ \\
\hline $\begin{array}{l}\text { O3S49E33CC } \\
03 S 50 E 11 D D \\
03 S 50 E 15 C B \\
03 S 50 E 31 C D B D \\
03551 E 03 D D\end{array}$ & $\begin{array}{l}16 N P R R \\
16-11 \\
1-B N \\
1 \text { NPRR } \\
16-3 N P\end{array}$ & $\begin{array}{r}10 \\
7 \\
12 \\
16 \\
6\end{array}$ & $\begin{array}{l}3510 \\
3256 \\
3288 \\
3399 \\
3199\end{array}$ & & & & & & 2863 & 130 & 2508 & 280 & 1915 & 0 & 1138 & 0 & 1010 & 0 & 703 & 0 & -324 & 0 \\
\hline $\begin{array}{l}03551 E 12 A A \\
03551 E 17 B B \\
03551 E 30 B B \\
03551 E 36 A A \\
03552 E 08 D D A C\end{array}$ & $\begin{array}{l}1-12 \\
4-17 \\
1 \text { GOVT } \\
36-2 \\
D-137\end{array}$ & $\begin{array}{r}7 \\
11 \\
11 \\
6 \\
10\end{array}$ & $\begin{array}{l}3257 \\
3173 \\
3244 \\
3106 \\
3121\end{array}$ & & & & & & 2936 & 135 & 2533 & 210 & 2093 & 0 & 1351 & 0 & 1234 & 0 & 914 & 5 & $\begin{array}{l}-311 \\
-159\end{array}$ & 0 \\
\hline $\begin{array}{l}\text { 03S53E O6AABD } \\
03554 E 02 B B A C \\
03554 E 12 A D A C \\
03554 E 29 B 8 \\
03555 E 13 B C\end{array}$ & $\begin{array}{l}6-1 \text { CRA } \\
2-1 \text { USA } \\
1 \text { IIMBE } \\
1-29 \\
1 \text { KREIT }\end{array}$ & $\begin{array}{r}5 \\
9 \\
0 \\
10 \\
0\end{array}$ & $\begin{array}{l}3013 \\
3105 \\
3088 \\
3172 \\
3072\end{array}$ & & & & & & & & 2901 & 200 & 2267 & 0 & 1540 & 0 & 1636 & 0 & 1078 & 5 & -12 & 0 \\
\hline $\begin{array}{l}\text { O3S56E } 12 D D \\
\text { O3S57E 05CDCA } \\
03557 E 32 C C \\
\text { 03S58E 02DC80 } \\
\text { 03S58E20DCCA }\end{array}$ & $\begin{array}{l}1-12 \\
1-5 \\
1-32 \\
1 F E D E R \\
D=103\end{array}$ & $\begin{array}{r}10 \\
7 \\
7 \\
11 \\
10\end{array}$ & $\begin{array}{l}3522 \\
3477 \\
3460 \\
3317 \\
3459\end{array}$ & & & & & & & & & & & & $\begin{array}{l}3168 \\
3179\end{array}$ & $\begin{array}{l}0 \\
0\end{array}$ & $\begin{array}{l}2997 \\
3014\end{array}$ & $\begin{array}{l}0 \\
0\end{array}$ & $\begin{array}{l}2629 \\
2642\end{array}$ & $\begin{array}{l}0 \\
5\end{array}$ & $\begin{array}{l}1644 \\
1680\end{array}$ & $\begin{array}{l}0 \\
0\end{array}$ \\
\hline $\begin{array}{l}\text { O3S59E25CC } \\
03 S 62 E 07 A B D B \\
04 S 15 E 03 D C B \\
04 S 16 E 368 B B C \\
\text { O4S17E19DBDB }\end{array}$ & $\begin{array}{l}D-116 \\
1-137 \\
1 \text { GOVT } \\
1-36 \\
A-1\end{array}$ & $\begin{array}{r}10 \\
8 \\
10 \\
11 \\
9\end{array}$ & $\begin{array}{l}3320 \\
4093 \\
5872 \\
5297 \\
4775\end{array}$ & 255 & & & 3531 & 60 & 3388 & 15 & 3222 & 155 & 2808 & $\theta$ & $\begin{array}{l}3100 \\
2076\end{array}$ & $\begin{array}{l}0 \\
0\end{array}$ & $\begin{array}{l}2910 \\
1921\end{array}$ & $\begin{array}{l}0 \\
0\end{array}$ & $\begin{array}{l}2542 \\
1589 \\
4208\end{array}$ & $\begin{array}{r}5 \\
10 \\
150\end{array}$ & $\begin{array}{r}1691 \\
701 \\
3628 \\
2879\end{array}$ & $\begin{array}{l}0 \\
0 \\
0 \\
0\end{array}$ \\
\hline
\end{tabular}


INTERVAL IUENTIFICATION

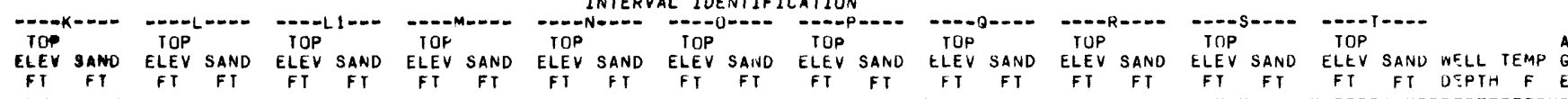

$\begin{array}{lllllll}-1173 & 20 & -1293 & 10 & -1661 & 100 & -2173\end{array}$

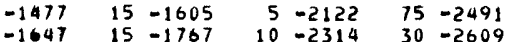

$25-2248 \quad 140-2459$ $\begin{array}{rlll}5 & -2581 & 140 & -2800\end{array}$

$$
-2778
$$

$\begin{array}{llllll}-1424 & 15-1502 & 10 & -2112 & 35 & -2357\end{array}$

$\begin{array}{rrr}-1349 & 10 & -1439 \\ -1234 & 5 & -1337\end{array}$

$\begin{array}{rlll}10 & -2112 & 35 & -2357 \\ 0 & -2038 & 45 & -2284 \\ 5 & -1933 & 25 & -2170\end{array}$

$10-1602$

$10-1820$

$5 \quad-873$

$10-1495$

$5-1619$

$10-1231$

$5-1431$

$0-1346$
$0-968$

$0 \begin{array}{ll}-1148 \\ 0\end{array}$

$\begin{array}{rr}0 & -968 \\ 0 & -30\end{array}$

$698 \quad 15 \quad 606$

$\begin{array}{llll}850 \quad 70 \quad 680 & 45\end{array}$

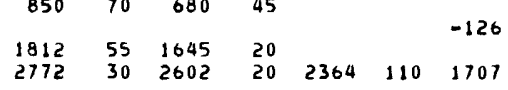

$65-2481 \quad 135-2720$

$\begin{array}{llll}70 & -2419 & 135 & -2644\end{array}$

$\begin{array}{llll}5 & -2280 & 126 & -2535 \\ -1997 & 128 & -2262\end{array}$

$\begin{array}{lll}-1997 & 128 & -2262 \\ -1704 & 120 & -2217\end{array}$

$\begin{array}{llll}35 & -1969 & 122 & -2229\end{array}$

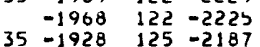

$\begin{array}{llll}35 & -1928 & 125 & -2187 \\ -1741 & 129 & -2058\end{array}$

$30-1556 \quad 120-1830$

$\begin{array}{lll}-1715 & 127 & -2007\end{array}$

$\begin{array}{lll}25 & -1453 & 120 \\ 35 & -1059 & 115\end{array}$

20

$\begin{array}{ll}15 & 4612\end{array}$

$10-1121$
-899
-854
-754
-499
-466

$\begin{array}{rrrrrrrr}777 & 15 & 073 & 0 & 112 & 0 & -55 & 250 \\ 369 & 20 & 269 & 0 & -226 & 0 & -396 & 20 \\ 182 & 15 & 70 & 0 & -390 & 0 & -547 & 75 \\ -399 & 15 & -499 & 0 & -964 & 0 & -1115 & 6502 \\ 1548 & 90 & 1365 & 55 & 1111 & 245 & 450 & 150\end{array}$

$\begin{array}{rrrr}-1741 & 129 & -2058 \\ -1814 & 120 & -2087\end{array}$

$$
\begin{array}{rrrrrrrrrr}
30 & -197 & 70 & -458 & 95 & -586 & 5 & -606 & 96 & \\
& -101 & 82 & -386 & 75 & -507 & 5 & -538 & 103 & -641 \\
& & & & & & & -521 & 76 & \\
25 & -124 & 66 & -383 & 60 & -448 & 20 & -577 & 70 &
\end{array}
$$

$\begin{array}{lllllllll}25 & -124 & 66 & -383 & 60 & -448 & 20 & -577 & 70\end{array}$

$\begin{array}{lllllllll}75 & -656 & 55 & -895 & 45 & -955 & 10 & -1126 & 30\end{array}$

$\begin{array}{rrrrrrrr}15 & 4612 & 60 & 4424 & 80 & 4280 & 110 & 4080 \\ 10 & 392 & 67 & 238 & 140 & 66 & 0 & -62\end{array}$

$\begin{array}{rrrr}67 & 238 & 140 & 66 \\ 70 & -1301 & 110 & -1431\end{array}$

$\begin{array}{rr}0 & -62 \\ 35 & -1606\end{array}$

95
65

$\begin{array}{lllllllll}0 & -165 & 80 & -546 & 80 & -673 & 0 & -826 & 40\end{array}$

$\begin{array}{lllll}15 & 1672 & 98 & 1482 & 50\end{array}$

$4426 \quad 70 \quad \begin{aligned} & 367 \\ & 390 \\ & 2390\end{aligned}$

2390

$\begin{array}{lllll}10 & 3657 & 105 & 3458 & 90\end{array}$

$10 \quad 2378 \quad 149 \quad 2121$

$\begin{array}{llll}5 & 2563 & 140 & 2317\end{array}$

$50 \quad 3554$

$70 \quad 2036$

$\begin{array}{lllll}-529 & 149 & -760 & \text { So } & -845\end{array}$

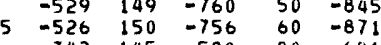

$\begin{array}{lllllll}617 & 20 & 516 & 50 & 70 & 105 & -514\end{array}$

$\begin{array}{lll}-342 \quad 145 & -580\end{array}$

$90-68$

$\begin{array}{llllll}-1757 \quad 15 & -1879 \quad 15 & -2399 & 55 & -2787\end{array}$

$\begin{array}{lllllll}-1667 & 15 & -1784 & 15 & -2354 & 45 & -2631\end{array}$

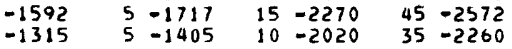

$\begin{array}{llll}25 & -2848 & 140 & -3069\end{array}$

$45-3127$

$\begin{array}{lllll}50 & -2752 \quad 163 & -2977\end{array}$

$\begin{array}{lllll}70 & -2694 & 133 & -2927\end{array}$

$\begin{array}{ll}55 & -3048 \\ 75 & -3029\end{array}$

$\begin{array}{rrrrr}-2380 & 126 & -2622 & 100 & -2733 \\ -2472 & 131 & -2725 & 90 & -2822\end{array}$

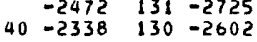

$75-2708$

$\begin{array}{lllll}-2378 & 130 & -2625 & 105 & -2730\end{array}$

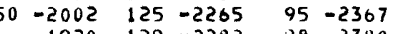

$\begin{array}{lllll}-1920 & 129 & -2282 & 98 & -2380 \\ -2235 & 124 & -2485 & 110 & -2596\end{array}$

$\begin{array}{lllll}-2045 & 123 & -2315 & 100 & -2415\end{array}$

$15-2513$

$-1874 \quad 126-2136$

$\begin{array}{lllllll}-914 & 0 & -1036 & 10 & -1657 & 10 & -1884\end{array}$

$\begin{array}{lllllll}-746 \quad 5 & -859 & 10 & -1494 & 10 & -1704\end{array}$

$\begin{array}{lllllll}-610 & 10 & -747 & 10 & -1373 & 10 & -1588\end{array}$

$\begin{array}{llllll}-347 \quad 10 & -498 \quad 5-1121 \quad 5 & -1313\end{array}$

$98-2236$

$\begin{array}{lll}-1991 \quad 128 & -2246 \\ -1989 & -2285\end{array}$

85

$-1921 \quad 121-2189$

$80-2275$

$15-2398$

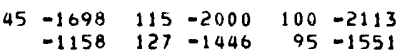

$\begin{array}{lllll}-1158 & 127 & -1446 & 95 & -1551 \\ -1014 & 129 & -1330 & 75-145\end{array}$

$\begin{array}{llllll}65 & -1443 & 134 & -1750 \quad 95 & -1845\end{array}$

$\begin{array}{lll}-778 & 94-1048 & 140-1188\end{array}$

8

$\begin{array}{llll}-256 & 60 & -416\end{array}$

$18 \quad-434$

$\begin{array}{llll}73 & -257 & & -34 \\ 65 & -34 & 105 & -170\end{array}$

$\begin{array}{lllllll}1159 & 5 & 1045 & 0 & 469 & 0 & 293\end{array}$

$\begin{array}{rrrrrrr}925 & 10 & 795 & 0 & 270 & 0 & 105 \\ -19 & 15 & -149 & 0 & -608 & 0 & -762\end{array}$

$\begin{array}{llllll}2813 \quad 70 \quad 2653 & 65 & & & & \\ 1417 & 175 & 1794\end{array}$

$\begin{array}{llllllr}2813 & 70 & 2653 & 65 & 2417 & 175 & 1753 \\ 2089 & 95 & 1864 & 45 & 1634 & 215 & 981\end{array}$ $\begin{array}{rr}30 & 14 \\ & 144 \\ 30 & 230\end{array}$

$\begin{array}{rr}45 & 40 \\ 55 & -869\end{array}$

$10 \quad 1732$

51711 $\begin{array}{llll}70 & -230 & 80 & -31\end{array}$

$\begin{array}{rrrr}63 & -1122 & 50 & -1187 \\ 65 & 1577 & 115 & 1457\end{array}$

$\begin{array}{llll}65 & 1577 & 115 & 1457 \\ 70 & 1538 & 115 & 1398\end{array}$

$\begin{array}{rrrr}70 & 1538 & 115 & 1398 \\ 68 & 766 & 120 & 634\end{array}$ $\begin{array}{rrrr}20 & -202 & 100 & -303\end{array}$
$29-2625$

$15-2533$

$10-2330$

$20-2476$

$\begin{array}{ll}b & -2140 \\ 5 & -1595\end{array}$

$\begin{aligned} & 5 \\ & 0\end{aligned}-1595$

$\begin{array}{ll}0 & -1487 \\ 0 & -187\end{array}$

$15-1203$

\begin{tabular}{rr}
18 & -434 \\
0 & -336 \\
0 & -355 \\
\hline
\end{tabular}

$\begin{array}{lllll}0 & -389 & 55 & -451 & 20\end{array}$

$2877 \quad 55 \quad 2748 \quad 4$ 0

4474152

$\begin{array}{rrr}2715 & 100 & K \\ 2589 & 75 & K\end{array}$

$107483 \mathrm{~K}$

1178

$\begin{array}{llll}30 & 1652 & 60 & 1476\end{array}$

$70 \quad 1818$ 65 165

$\begin{array}{lll}60 & 2357 \quad 74 T\end{array}$

$4850 \mathrm{~J}$

$\begin{array}{llll}4328 & 114 & \mathrm{~J} \\ 3970 & 100 & \mathrm{~J}\end{array}$

$-2327 \quad 50-2542$

$6592152 \mathrm{~J}$

$6492140 \mathrm{~J}$

$9349168 \mathrm{~T}$

$6214131 \mathrm{~J}$

$6376149 \mathrm{~J}$
$6433126 \mathrm{~J}$

$6360126 \mathrm{~K}$

$5796130 \mathrm{~K}$

$7200146 \mathrm{~J}$

$9330165 \mathrm{~J}$

$5695118 \mathrm{~J}$

$5772125 \mathrm{~J}$

585013
$5612118 \mathrm{~J}$

$4979117 \mathrm{~K}$

$5299115 \mathrm{~J}$

$4785111 \mathrm{~J}$

$4643118 \mathrm{~J}$

$\begin{array}{llll}5117 & 103 & K \\ 7750 & 143 \mathrm{~J}\end{array}$

$3583115 \mathrm{~K}$

$5971163 \%$

$4068105 \mathrm{~J}$

$3883108 \mathrm{~J}$

$3971101 \mathrm{~J}$

$5418137 \mathrm{~J}$

$\begin{array}{llll}5949 & 80 \\ & & & \\ 4142 & 98 & \mathrm{~J} \\ & & & \\ 50 \quad 144 \quad 115 \quad 28 \quad 100 \quad 5560 & 98 & \mathrm{~J}\end{array}$

$\begin{array}{rrrr}0 & -1249 & 70 & -1321 \\ 70 & 1302 & 115 & 1187\end{array}$

$30 \quad 1258$

$15 \quad 1187$ 


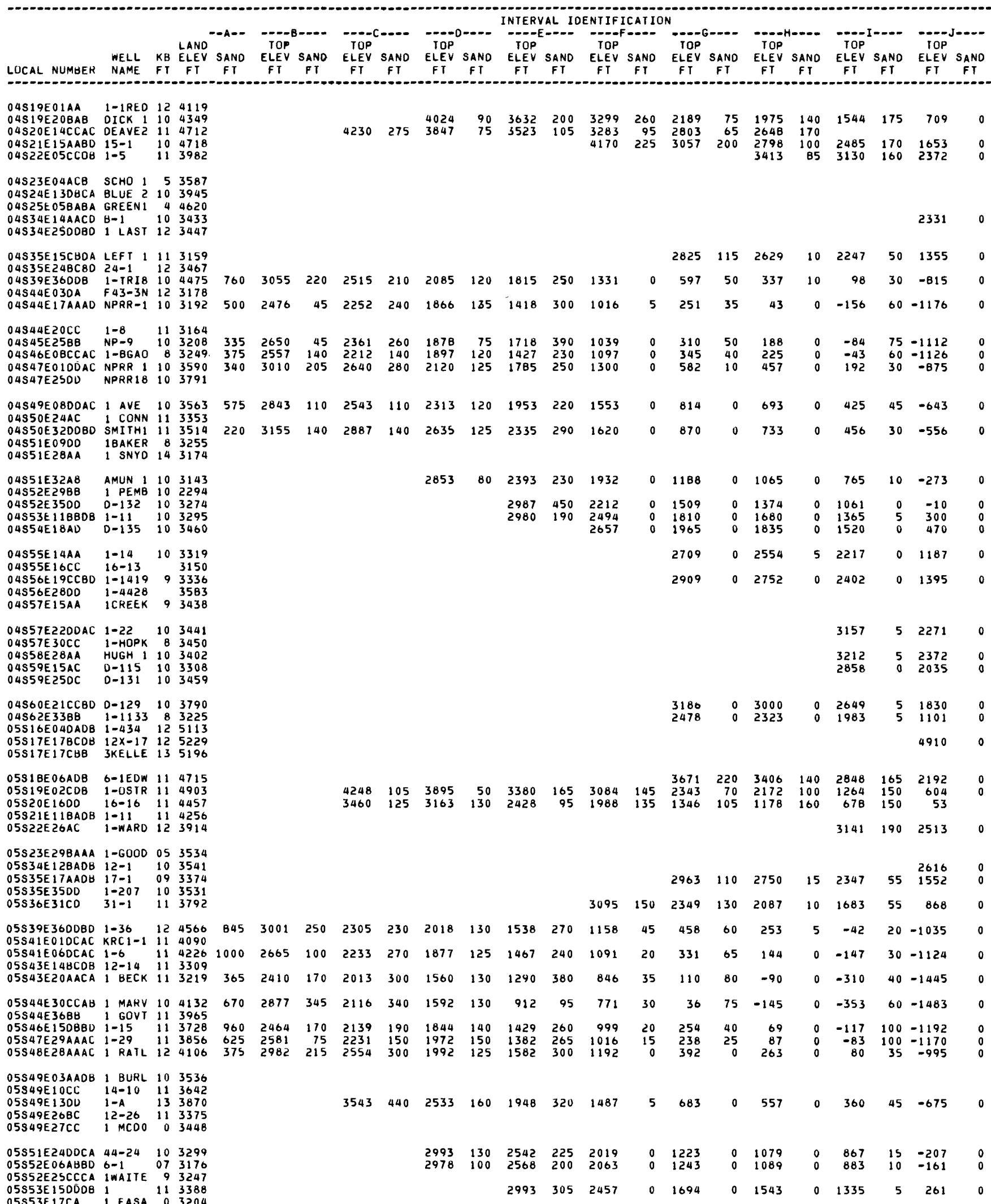


NTERVAL IUENTIFICAIION

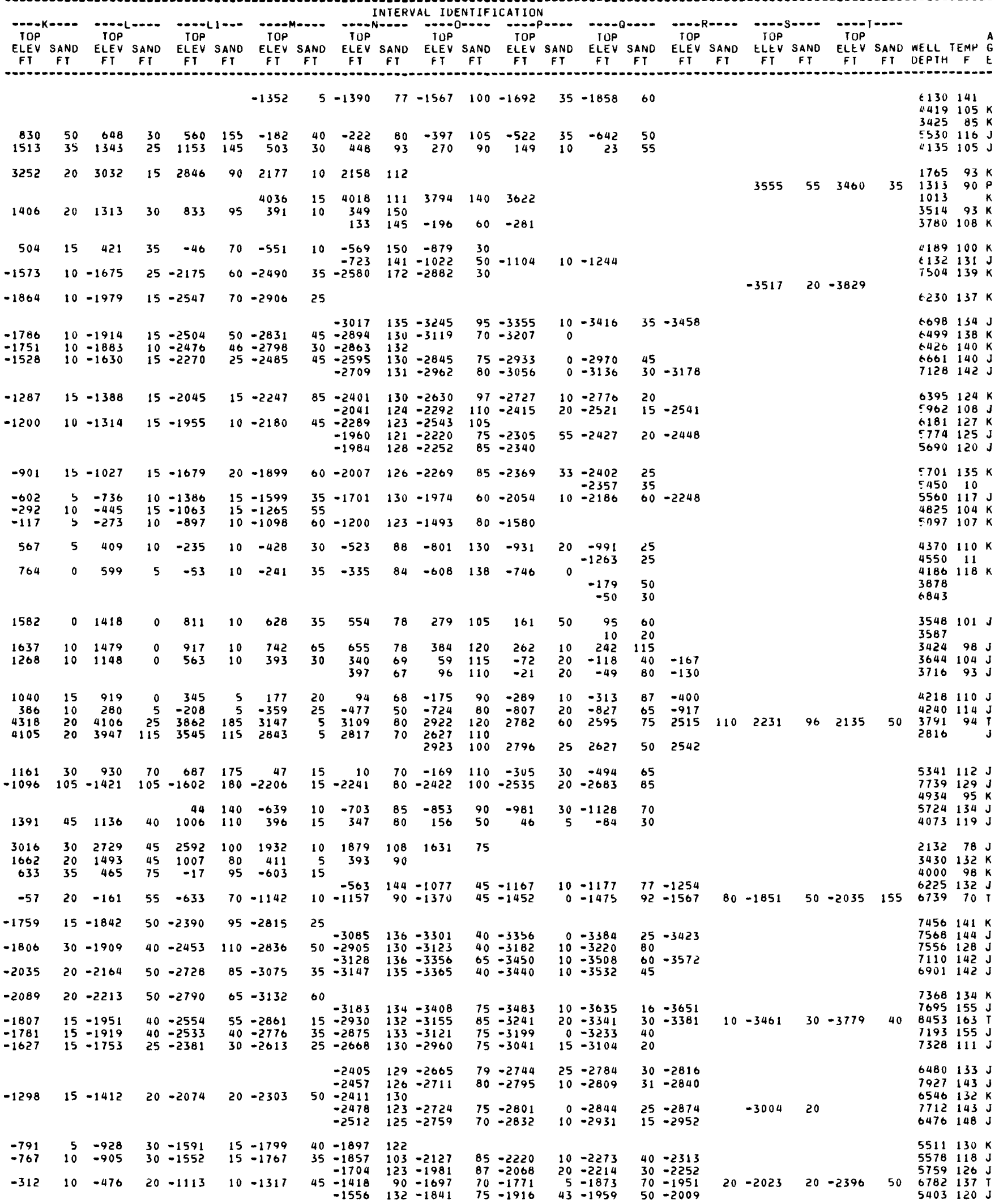


TABLE 2. -- SELECTED GEULOGIC DATA -- CONIINUEO

\begin{tabular}{|c|c|c|c|c|c|c|c|c|c|c|c|c|c|c|c|c|c|c|c|c|c|c|}
\hline LOCAL NUMBER & $\begin{array}{l}\text { WELLL } \\
\text { NAME }\end{array}$ & $\begin{array}{l}K B \\
F T\end{array}$ & $\begin{array}{l}\text { LAND } \\
\text { ELEV } \\
\text { FT }\end{array}$ & $\begin{array}{l}-A-D \\
\text { SAND } \\
\text { FT }\end{array}$ & $\begin{array}{l}\text { TOP } \\
\text { ELEV }\end{array}$ & $\begin{array}{l}\text { SAND } \\
\text { FT }\end{array}$ & $\begin{array}{l}\text { FI } \\
\text { ELEV } \\
--C\end{array}$ & $\begin{array}{l}\text { SAND } \\
\text { FT }\end{array}$ & $\begin{array}{l}\text { TOP } \\
\text { ELEV } \\
\text { FT }\end{array}$ & $\begin{array}{l}\text { SAND } \\
\text { FI }\end{array}$ & $\begin{array}{c}\text { TOP } \\
\text { ELEV } \\
\text { FT }\end{array}$ & $\begin{array}{l}\text { VAL ID } \\
\text { SAND } \\
\text { FT }\end{array}$ & $\begin{array}{l}\text { ENTIFI } \\
\text { ELEP } \\
\text { FLT }\end{array}$ & $\begin{array}{l}\text { SAND } \\
\text { FT }\end{array}$ & $\begin{array}{l}\text { TOP } \\
\text { ELEV } \\
\text { FT }\end{array}$ & $\begin{array}{l}\text { SAND } \\
\text { FT }\end{array}$ & $\begin{array}{l}\text { TOP } \\
\text { ELEV }\end{array}$ & $\begin{array}{c}\text { SAND } \\
\text { FT }\end{array}$ & $\begin{array}{c}\text { TOP } \\
\text { ELEV } \\
\text { FT }\end{array}$ & $\begin{array}{l}\text { SAND } \\
\text { FT }\end{array}$ & $\begin{array}{l}\text { FT } \\
\text { ELEP }\end{array}$ & $\begin{array}{l}\text { J SANO } \\
\text { Fi }\end{array}$ \\
\hline $\begin{array}{l}05554 E 01 C C \\
05 S 54 E 03 B C \\
05 S 54 E 16 A A \\
05554 E 25 C U A C \\
05555 E 01 B 8 C A\end{array}$ & $\begin{array}{l}1 F I T C H \\
1 \text { CALV } \\
1 \text { STATE } \\
1 \text { PANN } \\
1=1 \text { FED }\end{array}$ & $\begin{array}{r}9 \\
12 \\
11 \\
11 \\
8\end{array}$ & $\begin{array}{l}3242 \\
3369 \\
3404 \\
3703 \\
3292\end{array}$ & & & & & & & & & & 3095 & 0 & 2369 & 0 & 2213 & 0 & 2014 & 0 & 964 & 0 \\
\hline $\begin{array}{l}\text { 05S5SE 14AA } \\
\text { 05S55E25BB } \\
\text { 05S56E O2DAAC } \\
\text { 05S56E12BC } \\
\text { 05S57E23AD }\end{array}$ & $\begin{array}{l}1-\text { CROW } \\
1-25 \\
1-432 \\
D-102 \\
1 \text { TAUCK }\end{array}$ & $\begin{array}{l}08 \\
10 \\
11 \\
10 \\
06\end{array}$ & $\begin{array}{l}3577 \\
3384 \\
3487 \\
3496 \\
3401\end{array}$ & & & & & & & & & & & & 2735 & 0 & 2565 & 0 & 2348 & 0 & $\begin{array}{l}1305 \\
2401 \\
2727\end{array}$ & $\begin{array}{l}0 \\
0 \\
0\end{array}$ \\
\hline $\begin{array}{l}\text { 05S57E31AB } \\
05558 E 27 B B \\
05559 E 050 A A B \\
05559 E 17 C C \\
05560 E 21 C C\end{array}$ & $\begin{array}{l}\text { IWASH } \\
1 \text { CLEM } \\
D-114 \\
1-17 \\
21-13\end{array}$ & $\begin{array}{l}11 \\
06 \\
10 \\
07 \\
06\end{array}$ & $\begin{array}{l}3469 \\
3420 \\
3401 \\
3431 \\
3539\end{array}$ & & & & & & & & & & & & & & & & 3179 & 0 & $\begin{array}{l}2801 \\
2663 \\
2155\end{array}$ & $\begin{array}{l}0 \\
0 \\
0\end{array}$ \\
\hline 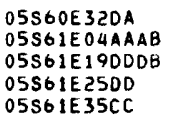 & $\begin{array}{l}1-32 \\
1-414 \\
1-4419 \\
14425 \\
1-1435\end{array}$ & $\begin{array}{r}11 \\
8 \\
08 \\
B \\
11\end{array}$ & $\begin{array}{l}3726 \\
3344 \\
3318 \\
3237 \\
3257\end{array}$ & & & & & & & & & & & & & & 3056 & 0 & 2918 & 5 & 1890 & 0 \\
\hline $\begin{array}{l}\text { 05S62E 19BCBC } \\
05 S 62 E 34 D C \\
06 S 17 E 09 C D \\
06 S 17 E 1408 \\
06 S 1 B E 0200\end{array}$ & $\begin{array}{l}1-1219 \\
1-3434 \\
10 S T R U \\
\text { MAC A1 } \\
2-16\end{array}$ & $\begin{array}{l}11 \\
10 \\
10 \\
11\end{array}$ & $\begin{array}{l}3258 \\
3289 \\
6184 \\
6003 \\
5057\end{array}$ & & & & & & & & & & & & 2853 & 195 & $\begin{array}{l}2686 \\
4610\end{array}$ & 150 & 2536 & 5 & $\begin{array}{l}1469 \\
4483 \\
3158\end{array}$ & $\begin{array}{l}0 \\
0 \\
0\end{array}$ \\
\hline $\begin{array}{l}\text { O6S1 BE 03BA } \\
06 S 1 B E 14 C B B \\
06 S 19 E 14 A C B D \\
06 S 20 E 14 D A D B \\
06 S 21 E 22 D D B D\end{array}$ & $\begin{array}{l}\text { 1SNYOE } \\
15-14 \\
7=14 \\
F O X 1 \\
16-22\end{array}$ & $\begin{array}{l}12 \\
10 \\
11 \\
13 \\
11\end{array}$ & $\begin{array}{l}5066 \\
5258 \\
4818 \\
4949 \\
4863\end{array}$ & $\begin{array}{l}315 \\
405 \\
405\end{array}$ & $\begin{array}{l}3419 \\
2842 \\
3886\end{array}$ & $\begin{array}{r}85 \\
130 \\
140\end{array}$ & $\begin{array}{l}2517 \\
1952 \\
2889\end{array}$ & $\begin{array}{l}210 \\
235 \\
275\end{array}$ & $\begin{array}{l}2080 \\
1312 \\
2374\end{array}$ & $\begin{array}{l}150 \\
120 \\
175\end{array}$ & $\begin{array}{r}1334 \\
720 \\
1684\end{array}$ & $\begin{array}{r}120 \\
90 \\
120\end{array}$ & $\begin{array}{r}989 \\
527 \\
1424\end{array}$ & $\begin{array}{l}280 \\
100 \\
125\end{array}$ & $\begin{array}{l}102 \\
-86 \\
699\end{array}$ & $\begin{array}{l}175 \\
277 \\
165\end{array}$ & $\begin{array}{r}-87 \\
-363 \\
469\end{array}$ & $\begin{array}{l}160 \\
185 \\
125\end{array}$ & 204 & 130 & $\begin{array}{r}-1468 \\
-1547 \\
-308\end{array}$ & $\begin{array}{l}0 \\
0 \\
0\end{array}$ \\
\hline $\begin{array}{l}06 S 21 E 31 C B \\
06 S 22 E 17 A B D A \\
06 S 23 E 13 C B C B \\
06 \$ 32 E 26 C 0 C A \\
06 S 32 E 27 B C C\end{array}$ & $\begin{array}{l}\text { 1ALBER } \\
2 X-17 \\
\text { MUTH } 1 \\
\text { TRIB 1 } \\
\text { IKNDWL }\end{array}$ & $\begin{array}{r}12 \\
12 \\
12 \\
09 \\
6\end{array}$ & $\begin{array}{l}5158 \\
4852 \\
3949 \\
3622 \\
3467\end{array}$ & 325 & 3585 & 80 & 2752 & 195 & 2384 & 70 & 1964 & 65 & 1834 & 135 & 1034 & 110 & 889 & 135 & 554 & 175 & 59 & 0 \\
\hline $\begin{array}{l}\text { O6S33E I BCCDC } \\
\text { O6S35E I7AACA } \\
\text { O6S36E OGBDAC } \\
\text { O6S36E 07ADBD } \\
\text { O6S36E } 16 D C\end{array}$ & $\begin{array}{l}A-1 \\
\text { TRIB } 1 \\
1 \text { YELL } \\
1 \text { BULL } \\
16-1\end{array}$ & $\begin{array}{l}10 \\
12 \\
10 \\
10 \\
10\end{array}$ & $\begin{array}{l}3656 \\
3579 \\
3600 \\
3711 \\
3682\end{array}$ & & & & & & & & & & 3222 & 110 & 2502 & 125 & 2238 & 20 & 1849 & 55 & 1097 & 0 \\
\hline $\begin{array}{l}\text { O6S36E } 17 D C \\
\text { O6S37E 1 OBCDA } \\
06 S 39 E 20 A D \\
06 S 40 E 23 B 8 \\
\text { O6S4IE13BBDB }\end{array}$ & $\begin{array}{l}1 \text { HAVE } \\
53-1 \\
1 \text { SHELL } \\
\text { SHELLB } \\
1 \text { EBEL }\end{array}$ & $\begin{array}{l}7 \\
09 \\
08 \\
11 \\
11\end{array}$ & $\begin{array}{l}3495 \\
4624 \\
3991 \\
3840 \\
3520\end{array}$ & $\begin{array}{l}430 \\
490 \\
580\end{array}$ & $\begin{array}{l}3811 \\
3164 \\
2844\end{array}$ & $\begin{array}{l}175 \\
225 \\
255\end{array}$ & $\begin{array}{l}3271 \\
2445 \\
2091\end{array}$ & $\begin{array}{l}260 \\
240 \\
280\end{array}$ & $\begin{array}{l}2898 \\
2099 \\
1691\end{array}$ & $\begin{array}{l}190 \\
160 \\
155\end{array}$ & $\begin{array}{l}2408 \\
1689 \\
1196\end{array}$ & $\begin{array}{l}225 \\
235 \\
220\end{array}$ & $\begin{array}{r}2038 \\
1287 \\
891\end{array}$ & $\begin{array}{l}65 \\
35 \\
35\end{array}$ & $\begin{array}{r}1352 \\
589 \\
162\end{array}$ & $\begin{array}{r}135 \\
100 \\
90\end{array}$ & $\begin{array}{r}1137 \\
369 \\
-29\end{array}$ & $\begin{array}{r}20 \\
0 \\
0\end{array}$ & $\begin{array}{r}86 \\
-281\end{array}$ & $\begin{array}{l}20 \\
20\end{array}$ & $\begin{array}{r}-805 \\
-1209\end{array}$ & : \\
\hline $\begin{array}{l}06 S 41 E 21 A A C A \\
06 S 42 E 12 B C \\
06 S 43 E 210 A C A \\
06 S 44 E 36 C C \\
06 S 45 E 04 B B B D\end{array}$ & $\begin{array}{l}41-21 \\
\text { VINCE I } \\
\text { BUNESI } \\
\text { IHORSE } \\
\text { BUCY I }\end{array}$ & $\begin{array}{l}11 \\
12 \\
10 \\
10 \\
10\end{array}$ & $\begin{array}{l}3543 \\
3299 \\
3306 \\
4048 \\
4037\end{array}$ & $\begin{array}{l}450 \\
290 \\
545 \\
760 \\
585\end{array}$ & $\begin{array}{l}2728 \\
2746 \\
2551 \\
2503 \\
2607\end{array}$ & $\begin{array}{l}190 \\
235 \\
350 \\
275 \\
205\end{array}$ & $\begin{array}{l}2022 \\
2075 \\
1860 \\
1948 \\
2152\end{array}$ & $\begin{array}{l}190 \\
290 \\
300 \\
170 \\
225\end{array}$ & $\begin{array}{l}1539 \\
1566 \\
1441 \\
1423 \\
1637\end{array}$ & $\begin{array}{r}125 \\
130 \\
90 \\
100 \\
80\end{array}$ & $\begin{array}{l}1149 \\
1171 \\
1166 \\
1038 \\
1267\end{array}$ & $\begin{array}{l}230 \\
200 \\
305 \\
290 \\
190\end{array}$ & $\begin{array}{l}704 \\
730 \\
596 \\
492 \\
805\end{array}$ & $\begin{array}{l}20 \\
15 \\
25 \\
20 \\
20\end{array}$ & $\begin{array}{r}-53 \\
-58 \\
-101 \\
-152 \\
64\end{array}$ & $\begin{array}{r}75 \\
40 \\
110 \\
80 \\
60\end{array}$ & $\begin{array}{l}-258 \\
-201 \\
-324 \\
-322 \\
-122\end{array}$ & $\begin{array}{l}0 \\
0 \\
0 \\
0 \\
0\end{array}$ & $\begin{array}{l}-486 \\
-440 \\
-532 \\
-538 \\
-321\end{array}$ & $\begin{array}{l}20 \\
30 \\
35 \\
50 \\
60\end{array}$ & $\begin{array}{l}-1396 \\
-1316 \\
-1590 \\
-1382\end{array}$ & $\begin{array}{l}0 \\
0 \\
0\end{array}$ \\
\hline $\begin{array}{l}\text { O6S45E 0 \&AA } \\
\text { O6S47E LODCAC } \\
06 S 48 E 20 B 8 \\
06 S 49 E 030 D \\
\text { O6S49E 23CBAC }\end{array}$ & $\begin{array}{l}1 \text { STAG } \\
\text { WHITEI } \\
\text { AMERAI } \\
\text { IRANCH } \\
1 \text { WITC }\end{array}$ & $\begin{array}{l}11 \\
12 \\
11 \\
10 \\
10\end{array}$ & $\begin{array}{l}4131 \\
3885 \\
4075 \\
3540 \\
3634\end{array}$ & $\begin{array}{l}665 \\
520 \\
310\end{array}$ & $\begin{array}{l}2752 \\
2856 \\
2950\end{array}$ & $\begin{array}{l}205 \\
140 \\
110\end{array}$ & $\begin{array}{l}2312 \\
2486 \\
2600\end{array}$ & $\begin{array}{l}170 \\
190 \\
140\end{array}$ & $\begin{array}{l}1882 \\
1951 \\
2155\end{array}$ & $\begin{array}{l}120 \\
110 \\
170\end{array}$ & $\begin{array}{l}1482 \\
1486 \\
1750\end{array}$ & $\begin{array}{l}340 \\
150 \\
300\end{array}$ & $\begin{array}{r}892 \\
984 \\
1217\end{array}$ & $\begin{array}{r}10 \\
10 \\
5\end{array}$ & $\begin{array}{l}127 \\
214 \\
450\end{array}$ & $\begin{array}{r}30 \\
10 \\
0\end{array}$ & $\begin{array}{r}-3 \\
64 \\
310\end{array}$ & $\begin{array}{l}5 \\
0 \\
0\end{array}$ & $\begin{array}{r}-203 \\
-102 \\
110\end{array}$ & $\begin{array}{l}95 \\
80 \\
35\end{array}$ & $\begin{array}{r}-1301 \\
-1193 \\
-937\end{array}$ & ? \\
\hline $\begin{array}{l}\text { O6S50EO3DB } \\
\text { O6S50E21BA } \\
\text { O6S50E30BABD } \\
\text { O6S50E35AAAC } \\
\text { O6S51E10AB }\end{array}$ & $\begin{array}{l}33-3 \\
\text { HURT } 1 \\
21-30 \\
1 \text { FRED } \\
1 \text { HORN }\end{array}$ & $\begin{array}{l}11 \\
10 \\
11 \\
10 \\
7\end{array}$ & $\begin{array}{l}3295 \\
3286 \\
3281 \\
3381 \\
3281\end{array}$ & 85 & 3121 & 125 & 2799 & 230 & 2231 & 130 & 1801 & 160 & 1456 & 5 & 667 & 0 & 528 & 0 & 328 & 35 & -714 & $\bullet$ \\
\hline $\begin{array}{l}06551 E 29 B B \\
06552 E 02 C 8 \\
06 S 52 E 06 A A \\
06552 E 07 C A B C \\
06 S 52 E 08 D 8\end{array}$ & $\begin{array}{l}29-4 \\
\text { IWAITE } \\
1 \text { STUV } \\
\text { NASH } 1 \\
\text { GOVT } 1\end{array}$ & $\begin{array}{r}07 \\
11 \\
7 \\
10 \\
12\end{array}$ & $\begin{array}{l}3333 \\
3084 \\
3324 \\
3330 \\
3386\end{array}$ & & & & 2975 & 140 & 2540 & 140 & 2040 & 290 & 1543 & 5 & 787 & 0 & 650 & 0 & 437 & 45 & -605 & - \\
\hline $\begin{array}{l}06 S 52 E 12 B D \\
06552 E 17 C B B U \\
06552 E 21 C C B D \\
06553 E 02 D A \\
06 S 53 E 05 D 0\end{array}$ & $\begin{array}{l}1 \text { ANOA } \\
\text { IKIRBY } \\
\text { I EAST } \\
\text { INISLE } \\
\text { I FEOE }\end{array}$ & $\begin{array}{l}11 \\
10 \\
12 \\
10 \\
10\end{array}$ & $\begin{array}{l}3174 \\
3377 \\
3259 \\
3246 \\
3182\end{array}$ & & & & & & 2944 & 150 & $\begin{array}{l}2507 \\
2901\end{array}$ & $\begin{array}{l}210 \\
150\end{array}$ & $\begin{array}{l}2048 \\
2338\end{array}$ & 5 & $\begin{array}{l}1273 \\
1589\end{array}$ & 0 & $\begin{array}{l}1135 \\
1433\end{array}$ & 0 & $\begin{array}{r}912 \\
1226\end{array}$ & $\begin{array}{l}25 \\
10\end{array}$ & $\begin{array}{r}-162 \\
151\end{array}$ & 0 \\
\hline
\end{tabular}

O6S53E13BBA 1 NISL 103158 $\begin{array}{lllll}\text { OOS53E1ODDAC } 1-16 & 10 & 3363 \\ \text { OOS53E I TCD } 1 \text { GOVT } 10 & 3352\end{array}$ OOSS3E2ZCC I NISL, 3362 


\begin{tabular}{|c|c|c|c|c|c|c|c|c|c|c|c|c|c|c|c|c|c|c|c|c|c|c|c|c|}
\hline $\begin{array}{l}\text { TOP } \\
\text { ELEV } \\
\text { FI }\end{array}$ & SAND & $\begin{array}{l}\text { TOP } \\
\text { ELEV } \\
\text { FT }\end{array}$ & $\begin{array}{l}\text { SAND } \\
\text { FT }\end{array}$ & $\begin{array}{l}\text { TOP } \\
\text { ELEV } \\
\text { FT }\end{array}$ & $\begin{array}{c}\text { SAND } \\
\text { FT }\end{array}$ & $\begin{array}{l}\text { TOP } \\
\text { ELEV } \\
\text { FT }\end{array}$ & $\begin{array}{l}\text { SAND } \\
\text { FT }\end{array}$ & $\begin{array}{l}\text { TOP } \\
\text { ELEV } \\
\text { FT }\end{array}$ & $\begin{array}{l}\text { SAND } \\
\text { FT }\end{array}$ & $\begin{array}{l}\text { TOP } \\
\text { ELEV } \\
F T\end{array}$ & $\begin{array}{c}\text { SAND } \\
\text { FI }\end{array}$ & $\begin{array}{l}\text { TUP } \\
\text { ELEV } \\
\text { FI }\end{array}$ & $\begin{array}{l}\text { SAND } \\
\text { FT }\end{array}$ & $\begin{array}{l}\text { IOP } \\
\text { ELEV } \\
F T\end{array}$ & $\begin{array}{l}\text { SAND } \\
\text { FI }\end{array}$ & $\begin{array}{l}\text { TOP } \\
\text { ELEV } \\
F T\end{array}$ & $\begin{array}{l}\text { SAND } \\
\text { FT }\end{array}$ & $\begin{array}{l}\text { TOP } \\
\text { ELEV } \\
\text { FT }\end{array}$ & $\begin{array}{l}\text { SAND } \\
\text { FI }\end{array}$ & $\begin{array}{l}\text { TOP } \\
\text { ELEV } \\
\text { FI }\end{array}$ & $\begin{array}{l}\text { SANO } \\
\text { FT }\end{array}$ & $\begin{array}{l}\text { WELL } \\
\text { DE }{ }^{\prime} T H\end{array}$ & TEMP & $\begin{array}{l}A \\
G \\
E\end{array}$ \\
\hline 399 & 5 & 249 & 20 & -393 & 10 & -592 & 40 & $\begin{array}{r}-1009 \\
-686 \\
-350\end{array}$ & $\begin{array}{l}90 \\
85 \\
84\end{array}$ & $\begin{array}{r}-1101 \\
-1269 \\
-1360 \\
-928 \\
-606\end{array}$ & $\begin{array}{r}85 \\
110 \\
100 \\
70 \\
135\end{array}$ & $\begin{array}{r}-1227 \\
-1379 \\
-1495 \\
-1029 \\
-745\end{array}$ & $\begin{array}{r}15 \\
50 \\
5\end{array}$ & $\begin{array}{r}-1266 \\
-1409 \\
-1513 \\
-1066 \\
-791\end{array}$ & $\begin{array}{l}25 \\
40 \\
45 \\
25 \\
35\end{array}$ & $\begin{array}{r}-1459 \\
-1095 \\
-829\end{array}$ & & & & & & $\begin{array}{l}4500 \\
4926 \\
4790 \\
4971 \\
4157\end{array}$ & $\begin{array}{lr}0 & \\
6 & 113 \\
0 & \\
1 & 98 \\
7 & 98\end{array}$ & $\begin{array}{l}\mathrm{J} \\
\mathrm{J} \\
\mathrm{J}\end{array}$ \\
\hline $\begin{array}{l}1754 \\
2033\end{array}$ & $\begin{array}{r}10 \\
5\end{array}$ & $\begin{array}{l}1588 \\
1872\end{array}$ & $\begin{array}{r}10 \\
5\end{array}$ & $\begin{array}{r}-135 \\
\\
958 \\
1230\end{array}$ & $\begin{array}{l}10 \\
10\end{array}$ & $\begin{array}{r}-321 \\
746 \\
1049\end{array}$ & $\begin{array}{l}40 \\
40\end{array}$ & $\begin{array}{r}-404 \\
-267 \\
581 \\
684 \\
975\end{array}$ & $\begin{array}{l}87 \\
83 \\
81 \\
80 \\
75\end{array}$ & $\begin{array}{r}-673 \\
-537 \\
309 \\
416\end{array}$ & $\begin{array}{r}80 \\
135 \\
135 \\
95\end{array}$ & $\begin{array}{r}-792 \\
-676 \\
168 \\
286\end{array}$ & $\begin{array}{r}0 \\
30 \\
15 \\
0\end{array}$ & $\begin{array}{r}-845 \\
-771 \\
119 \\
266\end{array}$ & $\begin{array}{l}35 \\
25 \\
50 \\
85\end{array}$ & $\begin{array}{l}-885 \\
-796\end{array}$ & 35 & -1077 & 40 & -1469 & 00 & $\begin{array}{l}4973 \\
4428 \\
3478 \\
3442 \\
2982\end{array}$ & $\begin{array}{lr}3 & 123 \\
8 & 111 \\
8 & 107 \\
2 & 90 \\
2 & 90\end{array}$ & $\begin{array}{l}\mathrm{T} \\
\mathrm{J} \\
\mathrm{J} \\
\mathrm{J} \\
\mathrm{K}\end{array}$ \\
\hline $\begin{array}{l}2080 \\
1932 \\
1405\end{array}$ & $\begin{array}{l}10 \\
10\end{array}$ & $\begin{array}{l}1874 \\
1734 \\
1206\end{array}$ & $\begin{array}{l}0 \\
0\end{array}$ & $\begin{array}{r}1326 \\
1163 \\
675\end{array}$ & $\begin{array}{l}5 \\
0\end{array}$ & $\begin{array}{r}1150 \\
994 \\
505\end{array}$ & $\begin{array}{l}35 \\
25\end{array}$ & $\begin{array}{r}1069 \\
713 \\
913\end{array}$ & $\begin{array}{l}75 \\
72 \\
70\end{array}$ & $\begin{array}{l}818 \\
438 \\
642\end{array}$ & $\begin{array}{l}85 \\
90\end{array}$ & $\begin{array}{l}347 \\
513\end{array}$ & $\begin{array}{r}15 \\
0\end{array}$ & $\begin{array}{l}285 \\
500\end{array}$ & $\begin{array}{r}80 \\
110\end{array}$ & $\begin{array}{l}204 \\
388\end{array}$ & 30 & 198 & & & & $\begin{array}{l}6462 \\
2859 \\
3381 \\
3323 \\
3308\end{array}$ & $\begin{array}{rr}2 & \\
9 & 92 \\
1 & 90 \\
3 & 100 \\
8 & 105\end{array}$ & $\begin{array}{l}k \\
j \\
J \\
k\end{array}$ \\
\hline 1150 & 15 & 960 & 0 & 471 & 0 & 306 & 15 & $\begin{array}{r}565 \\
-158 \\
252 \\
143\end{array}$ & $\begin{array}{l}64 \\
60 \\
55 \\
\\
62\end{array}$ & $\begin{array}{r}303 \\
-441 \\
-26 \\
-146\end{array}$ & $\begin{array}{r}100 \\
105 \\
85 \\
85\end{array}$ & $\begin{array}{r}182 \\
-548 \\
-136 \\
-273\end{array}$ & $\begin{array}{r}0 \\
10 \\
13\end{array}$ & $\begin{array}{r}157 \\
-568 \\
-149 \\
-515 \\
-290\end{array}$ & $\begin{array}{l}65 \\
80 \\
60 \\
92 \\
45\end{array}$ & $\begin{array}{r}87 \\
-652 \\
-220 \\
-345\end{array}$ & & & & & & $\begin{array}{l}3756 \\
4113 \\
3541 \\
3992 \\
3553\end{array}$ & 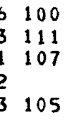 & $\begin{array}{l}\mathrm{J} \\
\mathrm{J} \\
\mathrm{J}\end{array}$ \\
\hline 776 & 20 & 596 & 0 & 156 & 0 & 1 & 25 & $\begin{array}{r}-91 \\
-139\end{array}$ & $\begin{array}{l}50 \\
51\end{array}$ & $\begin{array}{l}-356 \\
-424\end{array}$ & $\begin{array}{l}60 \\
65\end{array}$ & $\begin{array}{l}-460 \\
-548\end{array}$ & $\begin{array}{r}20 \\
3\end{array}$ & $\begin{array}{l}-524 \\
-571\end{array}$ & $\begin{array}{l}35 \\
30\end{array}$ & -630 & & 2486 & 82 & 2404 & & $\begin{array}{l}3942 \\
3995\end{array}$ & $\begin{array}{ll}2 & 116 \\
5 & 106\end{array}$ & $\mathrm{~J}$ \\
\hline $\begin{array}{l}3788 \\
2228\end{array}$ & $\begin{array}{l}25 \\
50\end{array}$ & $\begin{array}{l}3631 \\
2043\end{array}$ & $\begin{array}{l}30 \\
20\end{array}$ & $\begin{array}{l}3228 \\
1799\end{array}$ & $\begin{array}{r}90 \\
145\end{array}$ & $\begin{array}{l}2613 \\
1228\end{array}$ & $\begin{array}{l}10 \\
10\end{array}$ & $\begin{array}{l}2588 \\
1198\end{array}$ & $\begin{array}{l}70 \\
80\end{array}$ & $\begin{array}{l}2335 \\
1014\end{array}$ & $\begin{array}{r}170 \\
35\end{array}$ & $\begin{array}{r}2136 \\
908\end{array}$ & $\begin{array}{r}0 \\
35\end{array}$ & $\begin{array}{r}2053 \\
716\end{array}$ & $\begin{array}{l}90 \\
50\end{array}$ & & & & & & & $\begin{array}{l}4098 \\
4415\end{array}$ & $\begin{array}{ll}8 & 98 \\
5 & 96\end{array}$ & $\mathrm{~J}$ \\
\hline $\begin{array}{l}-2391 \\
-2016 \\
-1476\end{array}$ & $\begin{array}{l}45 \\
40 \\
70\end{array}$ & $\begin{array}{l}-2583 \\
-2243 \\
-1748\end{array}$ & $\begin{array}{l}50 \\
60 \\
75\end{array}$ & $\begin{array}{l}-2751 \\
-2518 \\
-2029\end{array}$ & $\begin{array}{l}235 \\
210 \\
200\end{array}$ & $\begin{array}{l}-3351 \\
-3141 \\
-2649\end{array}$ & $\begin{array}{l}15 \\
10 \\
10\end{array}$ & $\begin{array}{l}-3406 \\
-3158 \\
-2662\end{array}$ & $\begin{array}{l}90 \\
80 \\
90\end{array}$ & $\begin{array}{r}2090 \\
-1165 \\
-3546 \\
-3377 \\
-2894\end{array}$ & $\begin{array}{r}104 \\
100 \\
90 \\
75 \\
85\end{array}$ & $\begin{array}{r}1971 \\
-1282 \\
-3674 \\
-3501 \\
-3009\end{array}$ & $\begin{array}{l}30 \\
30 \\
25 \\
45 \\
30\end{array}$ & $\begin{array}{r}1788 \\
-1442 \\
-3836 \\
-3678 \\
-3156\end{array}$ & $\begin{array}{l}75 \\
98 \\
60 \\
50 \\
55\end{array}$ & $\begin{array}{r}1713 \\
-1540\end{array}$ & $\begin{array}{l}40 \\
30\end{array}$ & $\begin{array}{r}1450 \\
-1840\end{array}$ & $\begin{array}{l}92 \\
92\end{array}$ & 1358 & & $\begin{array}{l}8783 \\
8780 \\
5515\end{array}$ & $\begin{array}{ll}3 & 135 \\
0 & 144 \\
5 & 102\end{array}$ & $\begin{array}{l}\mathrm{J} \\
\mathrm{J} \\
\mathrm{J}\end{array}$ \\
\hline-718 & 85 & -898 & 75 & $\begin{array}{r}-1045 \\
3592\end{array}$ & $\begin{array}{l}60 \\
45\end{array}$ & $\begin{array}{r}-1366 \\
2957 \\
2846\end{array}$ & $\begin{array}{l}5 \\
5 \\
0\end{array}$ & $\begin{array}{r}-1385 \\
2945 \\
2831\end{array}$ & $\begin{array}{r}90 \\
80 \\
205\end{array}$ & $\begin{array}{r}-1875 \\
-1606 \\
2559\end{array}$ & $\begin{array}{l}100 \\
105 \\
65\end{array}$ & $\begin{array}{r}-1995 \\
-1721 \\
2441\end{array}$ & $\begin{array}{r}60 \\
25 \\
0\end{array}$ & $\begin{array}{r}-2196 \\
-1886 \\
2588 \\
2430\end{array}$ & $\begin{array}{l}64 \\
35 \\
25 \\
49\end{array}$ & $\begin{array}{r}-2260 \\
2552\end{array}$ & 50 & $\begin{array}{r}-2506 \\
2312 \\
2976\end{array}$ & $\begin{array}{l}75 \\
97 \\
55\end{array}$ & $\begin{array}{r}-2581 \\
2215 \\
2771\end{array}$ & 60 & $\begin{array}{l}6327 \\
2710 \\
3438\end{array}$ & $\begin{array}{rr}7 & 118 \\
0 & 85 \\
8 & 80\end{array}$ & $\begin{array}{l}\mathrm{J} \\
\mathrm{T} \\
\mathrm{T}\end{array}$ \\
\hline $\begin{array}{l}3381 \\
1941\end{array}$ & $\begin{array}{l}40 \\
25\end{array}$ & $\begin{array}{l}3272 \\
1843\end{array}$ & $\begin{array}{l}40 \\
30\end{array}$ & $\begin{array}{l}2812 \\
1364\end{array}$ & $\begin{array}{r}120 \\
80\end{array}$ & $\begin{array}{r}2221 \\
816\end{array}$ & $\begin{array}{l}5 \\
5\end{array}$ & $\begin{array}{r}2206 \\
802 \\
-850 \\
-929 \\
-1033\end{array}$ & $\begin{array}{l}200 \\
140 \\
137 \\
135 \\
140\end{array}$ & $\begin{array}{r}1918 \\
561 \\
-1072 \\
-1151 \\
-1258\end{array}$ & $\begin{array}{l}60 \\
45 \\
45 \\
30 \\
40\end{array}$ & $\begin{array}{r}1818 \\
463 \\
-1162 \\
-1232 \\
-1338\end{array}$ & $\begin{array}{r}0 \\
15 \\
20 \\
0 \\
10\end{array}$ & $\begin{array}{r}1796 \\
426 \\
-1183 \\
-1262 \\
-1368\end{array}$ & $\begin{array}{l}81 \\
90 \\
90 \\
75 \\
25\end{array}$ & $\begin{array}{r}1715 \\
336 \\
-1278 \\
-1349 \\
-1438\end{array}$ & $\begin{array}{l}35 \\
20\end{array}$ & $\begin{array}{r}1331 \\
76 \\
-1706\end{array}$ & $\begin{array}{l}30 \\
25\end{array}$ & $\begin{array}{r}1161 \\
-142 \\
-1918\end{array}$ & $\begin{array}{r}90 \\
130\end{array}$ & $\begin{array}{l}3937 \\
5962 \\
6451 \\
6496 \\
6925\end{array}$ & $\begin{array}{ll}7 & 100 \\
2 & 122 \\
1 & 128 \\
6 & 130 \\
5 & 125\end{array}$ & $\begin{array}{l}\mathrm{T} \\
\mathrm{T} \\
\mathrm{J} \\
\mathrm{J} \\
\mathrm{T}\end{array}$ \\
\hline $\begin{array}{l}-1633 \\
-2029\end{array}$ & $\begin{array}{l}15 \\
20\end{array}$ & $\begin{array}{l}-1734 \\
-2112\end{array}$ & $\begin{array}{l}25 \\
20\end{array}$ & $\begin{array}{l}-2278 \\
-2662\end{array}$ & $\begin{array}{r}70 \\
100\end{array}$ & $\begin{array}{l}-2731 \\
-3049\end{array}$ & $\begin{array}{l}10 \\
50\end{array}$ & $\begin{array}{l}-893 \\
-2751 \\
-3130 \\
-3305\end{array}$ & $\begin{array}{l}140 \\
140 \\
136 \\
131\end{array}$ & $\begin{array}{l}-1115 \\
-2971 \\
-3349 \\
-3521\end{array}$ & $\begin{array}{l}35 \\
40 \\
40\end{array}$ & $\begin{array}{l}-1190 \\
-3061 \\
-3442 \\
-3606\end{array}$ & $\begin{array}{r}30 \\
10 \\
0 \\
10\end{array}$ & $\begin{array}{l}-1225 \\
-3078 \\
-3508 \\
-3728\end{array}$ & $\begin{array}{l}85 \\
42 \\
30 \\
40\end{array}$ & $\begin{array}{l}-1313 \\
-3120 \\
-3775\end{array}$ & & & & & & $\begin{array}{l}6 ? 92 \\
4113 \\
7238 \\
7403 \\
7334\end{array}$ & $\begin{array}{ll}2 & 115 \\
3 & 113 \\
8 & 154 \\
3 & 145 \\
4 & 158\end{array}$ & $\begin{array}{l}\mathrm{J} \\
\mathrm{K} \\
\mathrm{J} \\
\mathrm{J} \\
\mathrm{J}\end{array}$ \\
\hline $\begin{array}{l}-2206 \\
-2147\end{array}$ & $\begin{array}{l}20 \\
10\end{array}$ & $\begin{array}{l}-2302 \\
-2247\end{array}$ & $\begin{array}{l}10 \\
15\end{array}$ & $\begin{array}{l}-2879 \\
-2844\end{array}$ & $\begin{array}{l}80 \\
40\end{array}$ & $\begin{array}{l}-3252 \\
-3199\end{array}$ & $\begin{array}{l}30 \\
25\end{array}$ & $\begin{array}{l}-3321 \\
-3269\end{array}$ & $\begin{array}{l}138 \\
138\end{array}$ & $\begin{array}{l}-3544 \\
-3489\end{array}$ & $\begin{array}{l}35 \\
40\end{array}$ & $\begin{array}{l}-3622 \\
-3578\end{array}$ & $\begin{array}{r}0 \\
10\end{array}$ & -3696 & 40 & & & & & & & $\begin{array}{l}7423 \\
7042 \\
4156\end{array}$ & $\begin{array}{ll}3 & 162 \\
2 & 150 \\
6 & 105\end{array}$ & $\begin{array}{l}J \\
k \\
k\end{array}$ \\
\hline $\begin{array}{l}-2297 \\
-2033\end{array}$ & $\begin{array}{r}10 \\
5\end{array}$ & $\begin{array}{l}-2422 \\
-2168\end{array}$ & $\begin{array}{l}15 \\
10\end{array}$ & $\begin{array}{l}-3001 \\
-2743\end{array}$ & $\begin{array}{l}35 \\
20\end{array}$ & $\begin{array}{l}-3342 \\
-3086\end{array}$ & $\begin{array}{l}25 \\
25\end{array}$ & -3390 & 132 & -3612 & 35 & -3680 & 5 & -3800 & 45 & & & & & & & $\begin{array}{l}7361 \\
7210\end{array}$ & $\begin{array}{ll}1 & 157 \\
0 & 141\end{array}$ & $\mathrm{~J}$ \\
\hline $\begin{array}{l}-1914 \\
-1786 \\
-1530\end{array}$ & $\begin{array}{l}10 \\
10 \\
10\end{array}$ & $\begin{array}{l}-2056 \\
-1944 \\
-1672\end{array}$ & $\begin{array}{l}10 \\
10 \\
15\end{array}$ & $\begin{array}{l}-2670 \\
-2604 \\
-2315\end{array}$ & $\begin{array}{l}25 \\
25 \\
15\end{array}$ & $\begin{array}{l}-2903 \\
-2186 \\
-2542\end{array}$ & $\begin{array}{l}35 \\
20 \\
20\end{array}$ & $\begin{array}{l}-3188 \\
-3013 \\
-2882 \\
-2643 \\
-2656\end{array}$ & $\begin{array}{l}138 \\
130 \\
130 \\
128 \\
130\end{array}$ & $\begin{array}{l}-3416 \\
-3244 \\
-3127 \\
-2888 \\
-2916\end{array}$ & $\begin{array}{l}45 \\
80 \\
70 \\
65 \\
85\end{array}$ & $\begin{array}{l}-3493 \\
-3206 \\
-3004\end{array}$ & $\begin{array}{r}0 \\
10 \\
5\end{array}$ & $\begin{array}{l}-3559 \\
-3284 \\
-3130\end{array}$ & $\begin{array}{l}60 \\
35 \\
16\end{array}$ & $\begin{array}{l}-3621 \\
-3322 \\
-3146\end{array}$ & & & & & & $\begin{array}{l}7826 \\
7315 \\
7212 \\
6585 \\
6900\end{array}$ & $\begin{array}{ll}6 & 143 \\
5 & 160 \\
2 & 158 \\
5 & 139 \\
135 & 135\end{array}$ & $\begin{array}{l}J \\
k \\
J \\
k \\
J\end{array}$ \\
\hline-1314 & 5 & -1454 & 15 & -2107 & 20 & -2336 & 40 & $\begin{array}{l}-2443 \\
-2425 \\
-2641 \\
-2409 \\
-1980\end{array}$ & $\begin{array}{l}125 \\
124 \\
126 \\
119 \\
121\end{array}$ & $\begin{array}{l}-2689 \\
-2658 \\
-2884 \\
-2648 \\
-2234\end{array}$ & $\begin{array}{r}90 \\
134 \\
10 \\
113 \\
110\end{array}$ & $\begin{array}{l}-2792 \\
-2965 \\
-2761 \\
-2344\end{array}$ & $\begin{array}{r}25 \\
0 \\
10 \\
25\end{array}$ & $\begin{array}{l}-2926 \\
-3008 \\
-2829 \\
-2426\end{array}$ & $\begin{array}{l}45 \\
18 \\
20 \\
10\end{array}$ & $\begin{array}{l}-2971 \\
-3026 \\
-2851 \\
-2438\end{array}$ & & & & & & $\begin{array}{l}6140 \\
6 \geqslant 99 \\
6400 \\
6 \geqslant 60 \\
5747\end{array}$ & $\begin{array}{ll}0 & 126 \\
9 & 136 \\
0 & 120 \\
0 & 130 \\
7 & 154\end{array}$ & $\begin{array}{l}\mathrm{K} \\
\mathrm{J} \\
\mathrm{J} \\
\mathrm{J} \\
\mathrm{J}\end{array}$ \\
\hline-1198 & 5 & -1338 & 10 & -1996 & 10 & -2216 & 25 & $\begin{array}{l}-1853 \\
-1907 \\
-1870 \\
-1852\end{array}$ & $\begin{array}{l}120 \\
122 \\
125 \\
120\end{array}$ & $\begin{array}{l}-2115 \\
-2166 \\
-2130 \\
-2112\end{array}$ & $\begin{array}{l}110 \\
100 \\
145 \\
115\end{array}$ & $\begin{array}{l}-2225 \\
-2269 \\
-2278 \\
-2228\end{array}$ & $\begin{array}{l}20 \\
20 \\
40 \\
35\end{array}$ & $\begin{array}{l}-2309 \\
-2351 \\
-2332 \\
-2315\end{array}$ & $\begin{array}{l}45 \\
40 \\
50 \\
40\end{array}$ & $\begin{array}{l}-2362 \\
-2392 \\
-2382 \\
-2360\end{array}$ & & & & & & $\begin{array}{l}5486 \\
5543 \\
5905 \\
9700 \\
5987\end{array}$ & $\begin{array}{l}119 \\
3118 \\
112 \\
168 \\
113\end{array}$ & $\begin{array}{l}x \\
j \\
j \\
j \\
J\end{array}$ \\
\hline $\begin{array}{l}-713 \\
-409\end{array}$ & 10 & $\begin{array}{l}-858 \\
-560\end{array}$ & $\begin{array}{l}10 \\
10\end{array}$ & $\begin{array}{l}-1521 \\
-1246\end{array}$ & $\begin{array}{l}10 \\
15\end{array}$ & $\begin{array}{l}-1730 \\
-1444\end{array}$ & $\begin{array}{l}40 \\
30\end{array}$ & $\begin{array}{l}-1788 \\
-1833 \\
-1849 \\
-1711\end{array}$ & $\begin{array}{r}119 \\
94 \\
123 \\
125\end{array}$ & $\begin{array}{l}-2054 \\
-2075 \\
-2102 \\
-1988\end{array}$ & $\begin{array}{l}115 \\
115 \\
125 \\
80\end{array}$ & $\begin{array}{l}-2173 \\
-2195 \\
-2227 \\
-2074\end{array}$ & $\begin{array}{l}40 \\
15 \\
30\end{array}$ & $\begin{array}{l}-2275 \\
-2263 \\
-2294\end{array}$ & $\begin{array}{l}59 \\
40 \\
45\end{array}$ & $\begin{array}{l}-2334 \\
-2313 \\
-2359\end{array}$ & & & & & & $\begin{array}{l}5541 \\
5740 \\
5441 \\
4807 \\
5315\end{array}$ & $\begin{array}{ll}1 & 122 \\
0 & 135 \\
1 & 125 \\
7 & 114 \\
5 & 119\end{array}$ & $\begin{array}{l}J \\
J \\
J \\
k \\
k\end{array}$ \\
\hline & & & & & & & & $\begin{array}{l}-1465 \\
-1602 \\
-1688 \\
-1578 \\
-1269\end{array}$ & $\begin{array}{l}110 \\
90 \\
128 \\
111 \\
115\end{array}$ & $\begin{array}{l}-1724 \\
-1858 \\
-1458 \\
-1839 \\
-1542\end{array}$ & $\begin{array}{r}95 \\
140 \\
100 \\
110 \\
108\end{array}$ & $\begin{array}{l}-1824 \\
-2003 \\
-2063 \\
-1951 \\
-1650\end{array}$ & $\begin{array}{l}35 \\
20 \\
50 \\
15 \\
30\end{array}$ & $\begin{array}{l}-1892 \\
-2047 \\
-2162 \\
-2018 \\
-1697\end{array}$ & $\begin{array}{l}45 \\
35 \\
45 \\
40 \\
65\end{array}$ & $\begin{array}{l}-1947 \\
-2102 \\
-2208 \\
-2059 \\
-1774\end{array}$ & & & & & & $\begin{array}{l}5262 \\
5484 \\
5800 \\
5434 \\
5069\end{array}$ & $\begin{array}{ll}2114 \\
4 \\
1 \\
119 \\
0 & 122 \\
4 & 117 \\
9 & 118\end{array}$ & $\begin{array}{l}\mathrm{J} \\
\mathrm{J} \\
\mathrm{J} \\
\mathrm{J} \\
\mathrm{J}\end{array}$ \\
\hline
\end{tabular}


TABLE 2. - SELECTED GEOLOGIC DATA -- CONTINUED

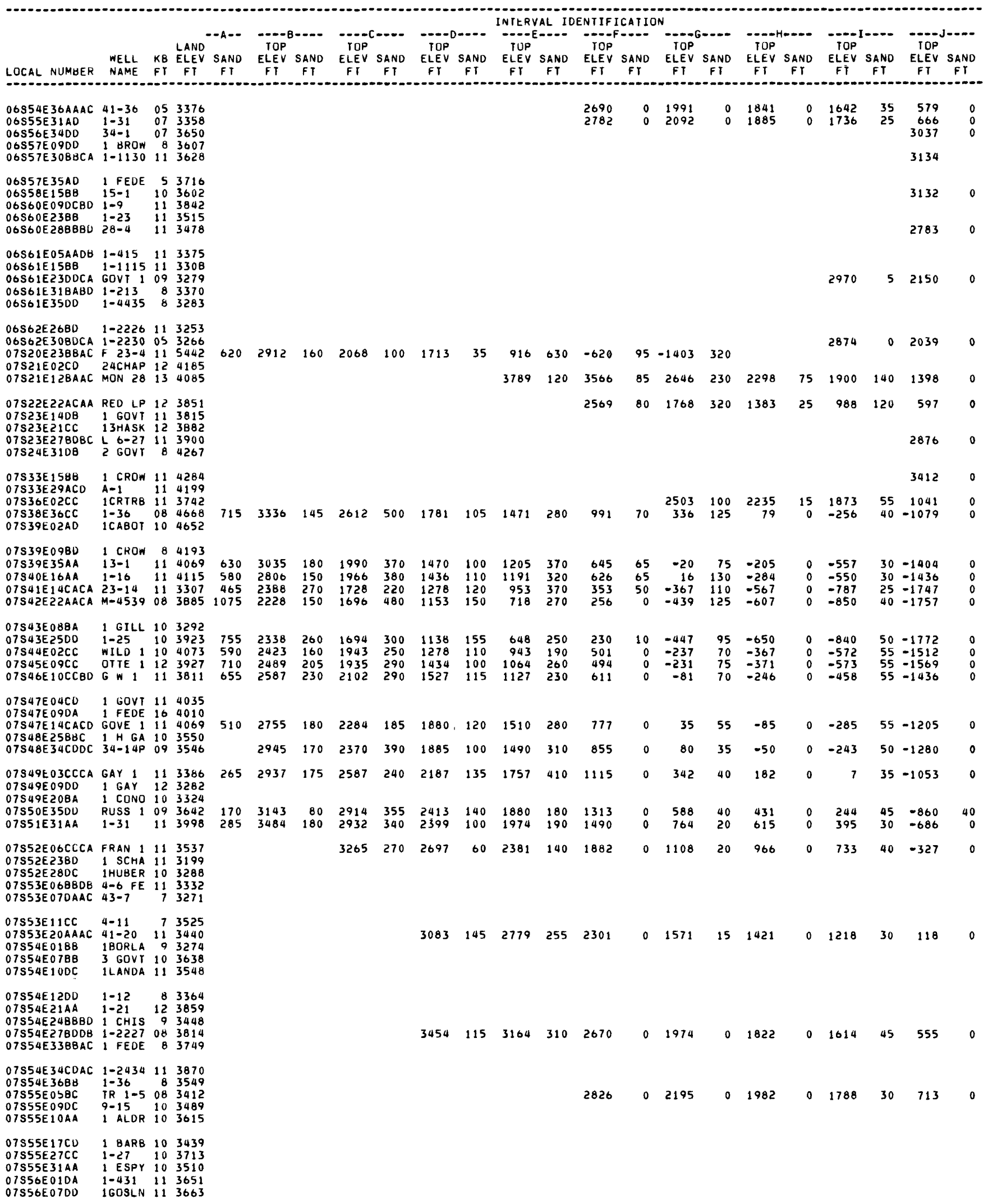


INTERVAL IOENTIFICATION

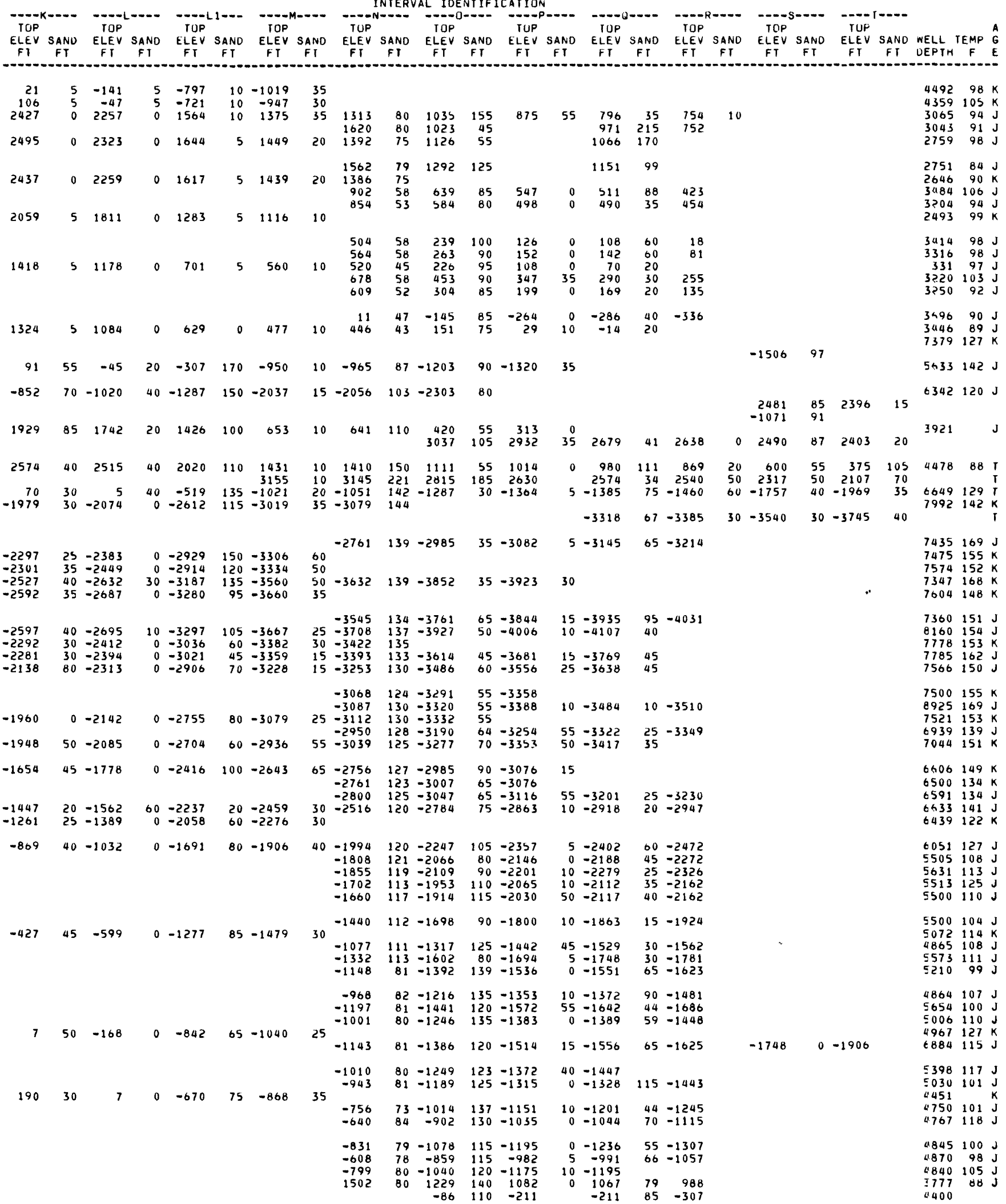


table 2. -- SELECTED geologic data -- CONTINUED

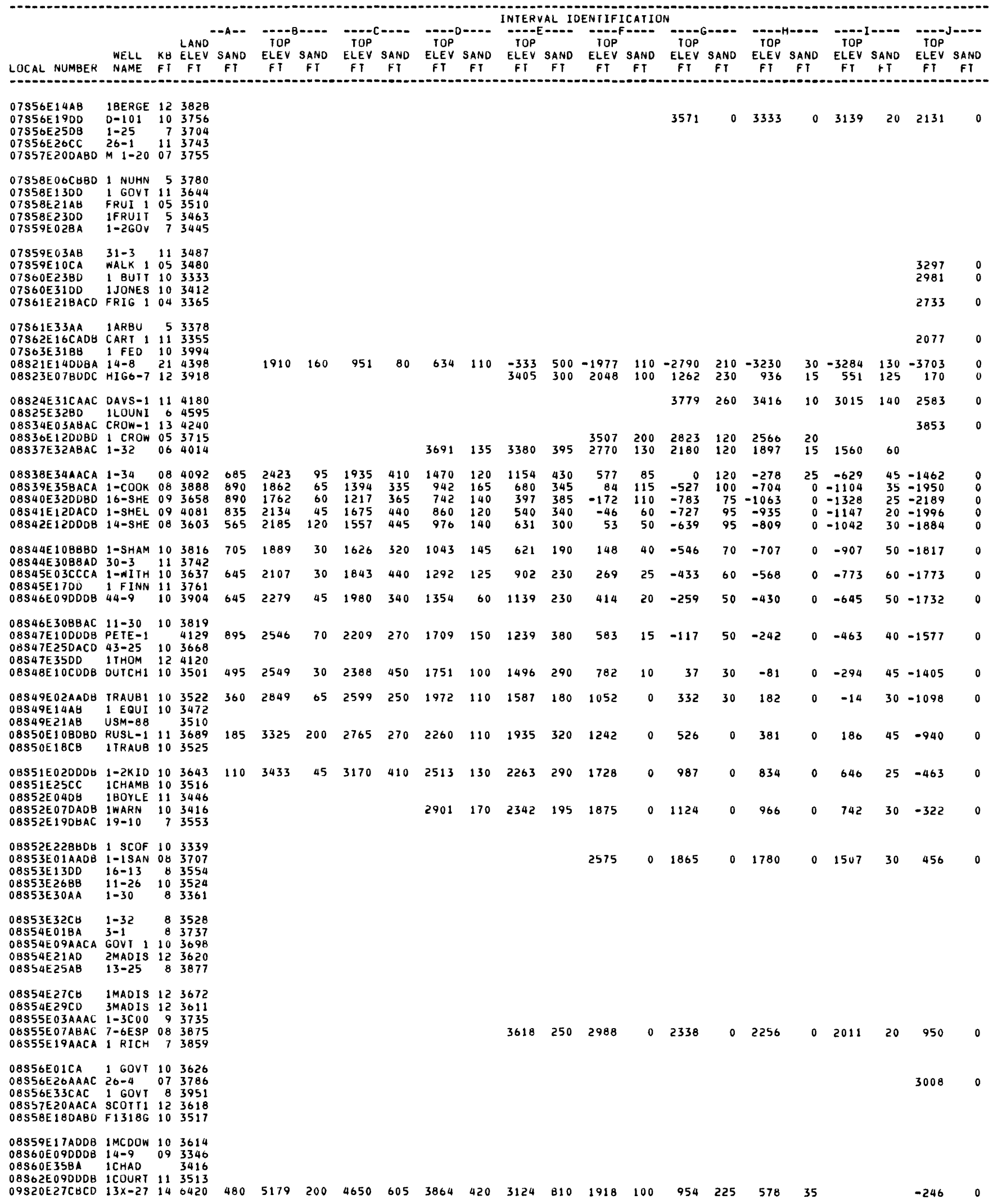




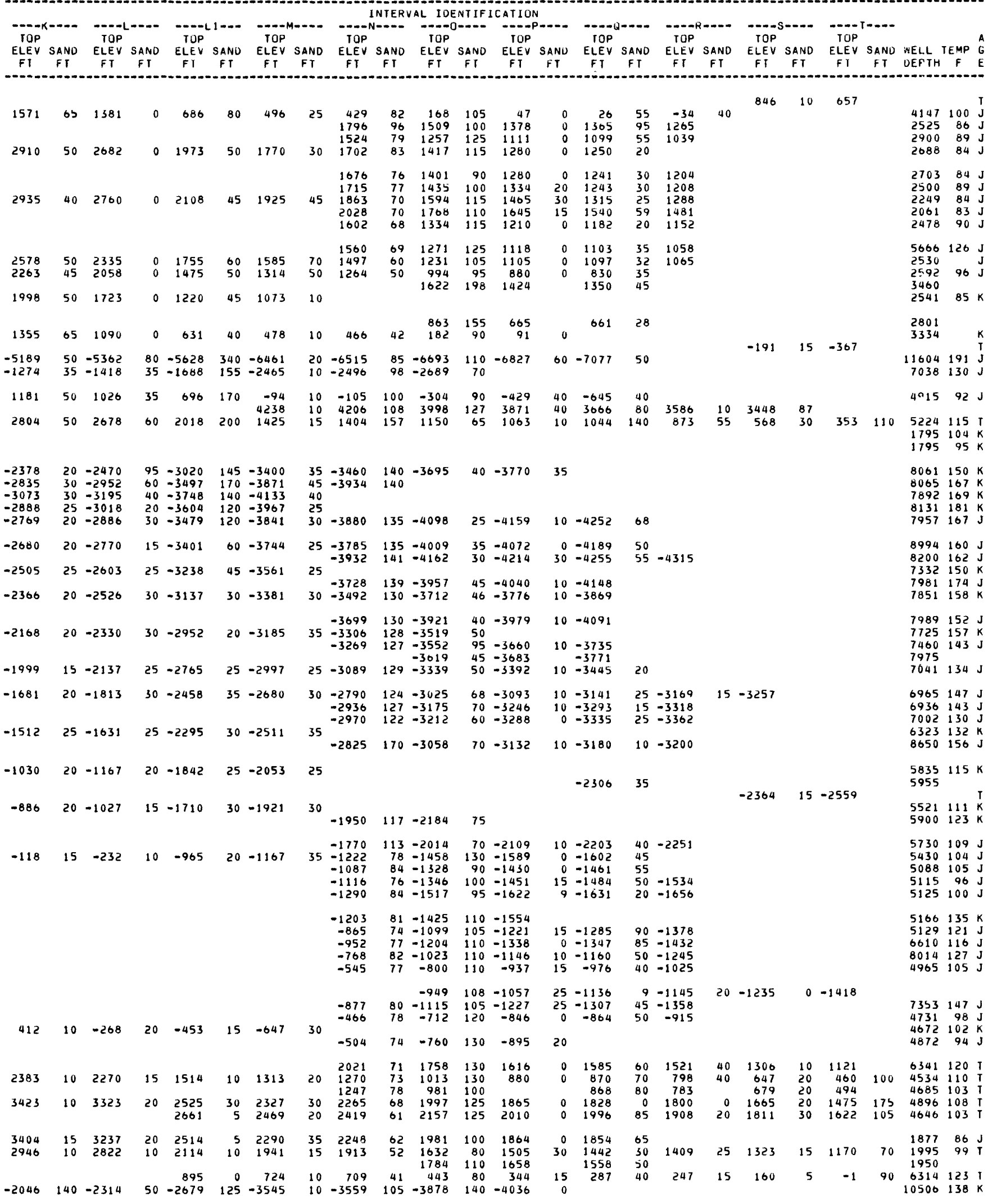


TABLE 2. -- SELECTEU GEOLOGIC DATA -- CONTINUED

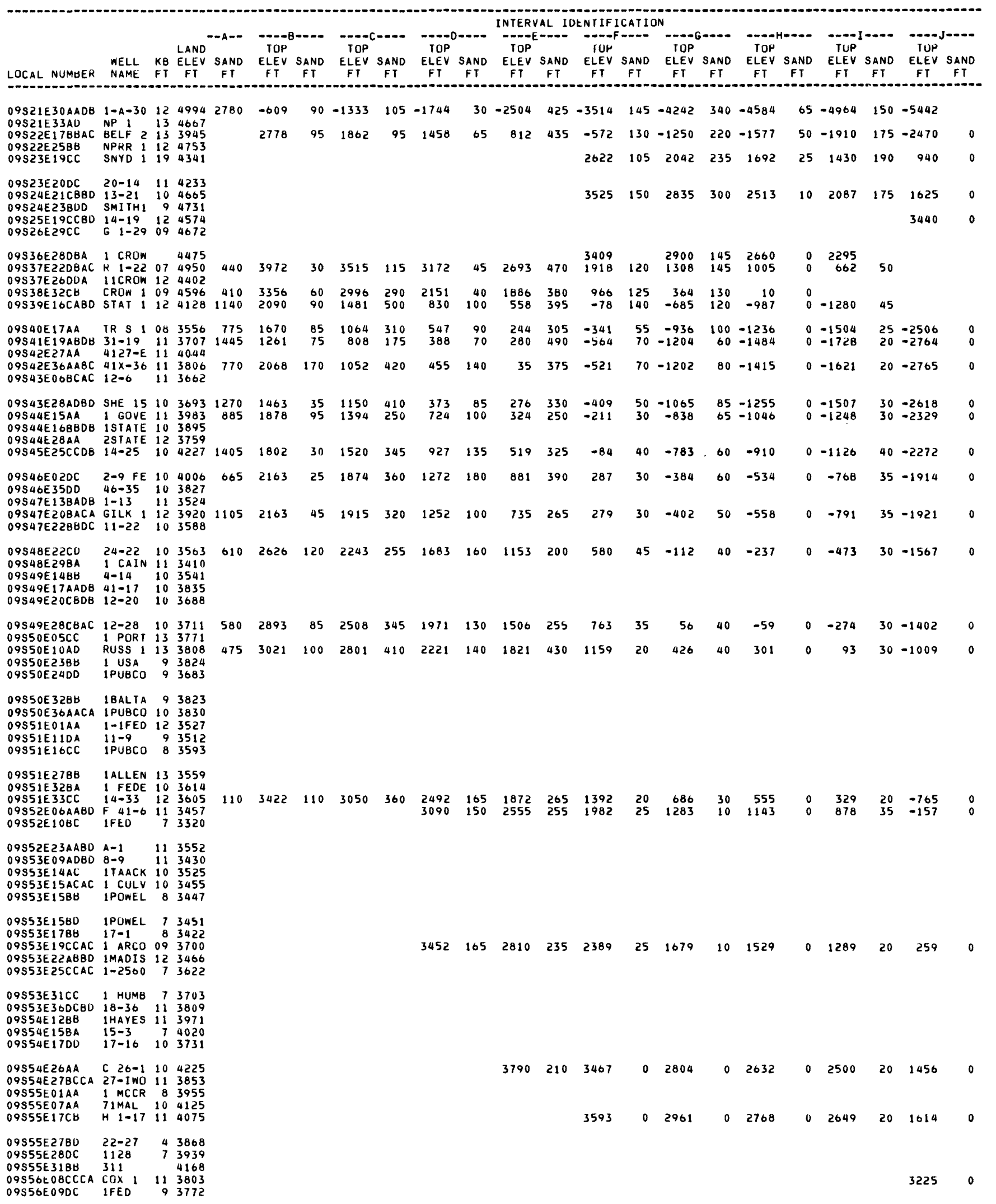




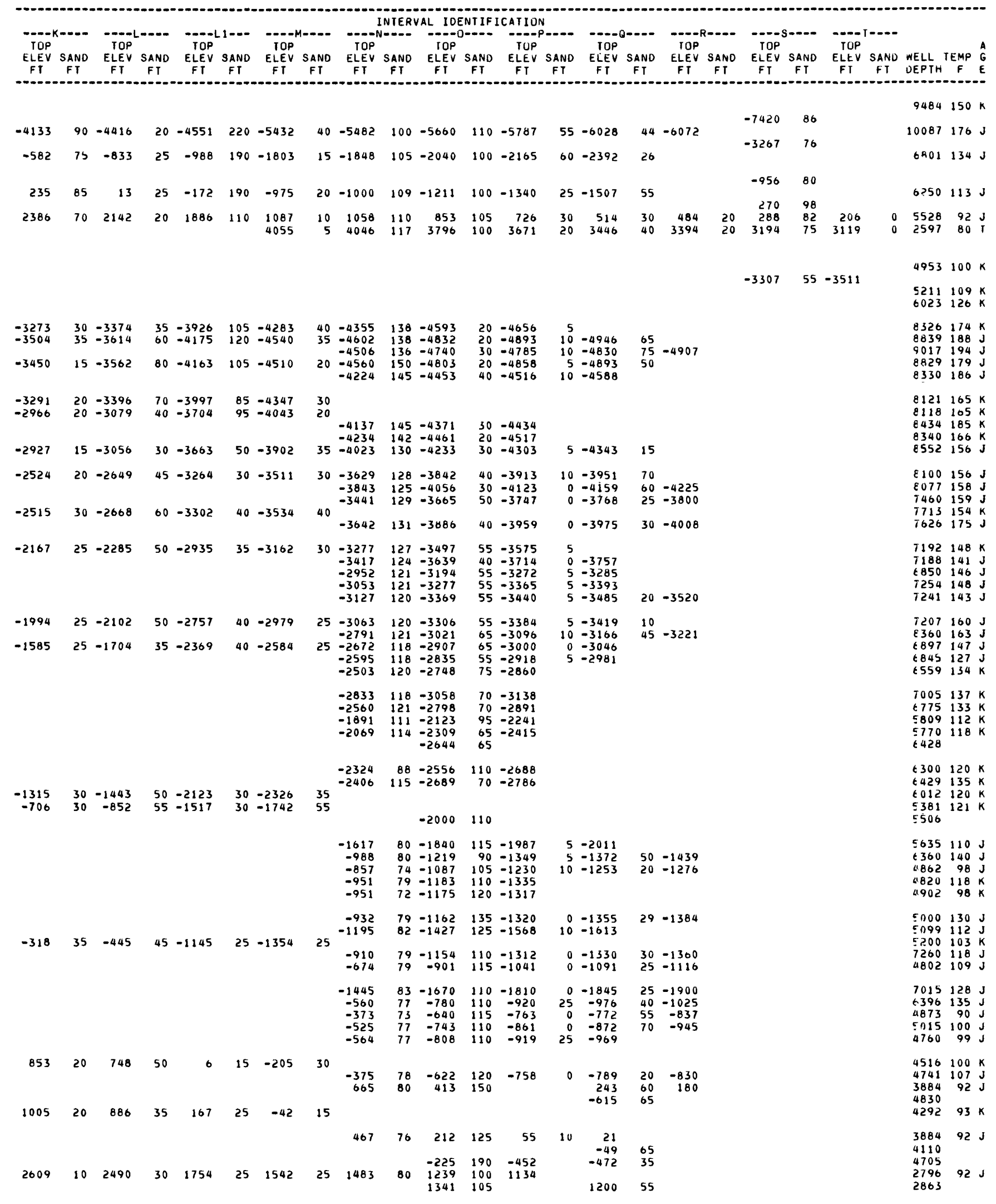


TABLE 2. -- SELECTED GEOLUGIC DATA -- CONTINUED

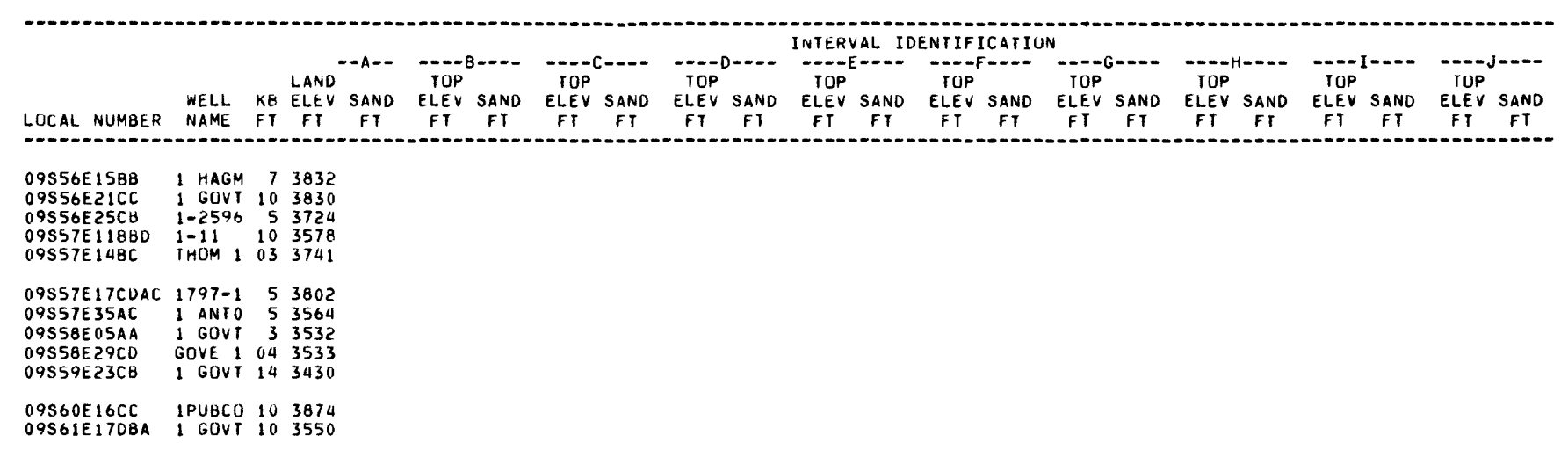


INIERVAL IOENTIFICATION

\begin{tabular}{|c|c|c|c|c|c|c|c|c|c|c|c|c|c|c|c|c|c|c|c|c|c|c|c|}
\hline $\begin{array}{l}\text { TOP } \\
\text { ELEV SAND } \\
\text { FT FT }\end{array}$ & $\begin{array}{l}--=-1 \\
\text { IOP } \\
\text { ELEV } \\
\text { FT }\end{array}$ & $\begin{array}{l}\text { SAND } \\
\text { FT }\end{array}$ & $\begin{array}{c}----1 \\
\text { TOP } \\
\text { ELEV } \\
\text { FT }\end{array}$ & $\begin{array}{c}\text { SAND } \\
\text { FT }\end{array}$ & $\begin{array}{l}-=-1 \\
\text { TOP } \\
\text { ELEV }\end{array}$ & $\begin{array}{l}\text { SAND } \\
\text { FT }\end{array}$ & $\begin{array}{l}\text { TOP } \\
\text { ELEV } \\
\text { FI }\end{array}$ & $\begin{array}{l}\text { N-E=- } \\
\text { SAND } \\
\text { F T }\end{array}$ & $\begin{array}{l}\text { ELEV } \\
\text { ETOP } \\
\text { TI }\end{array}$ & $\begin{array}{c}\text { SAND } \\
\text { FT }\end{array}$ & $\begin{array}{l}\text { FT } \\
\text { ELEV }\end{array}$ & $\begin{array}{l}\text { SAND } \\
\text { FI }\end{array}$ & $\begin{array}{l}\text { TOP } \\
\text { ELEV } \\
\text { FT }\end{array}$ & $\begin{array}{c}\text { SAND } \\
\text { FI }\end{array}$ & $\begin{array}{l}\text { IUP } \\
\text { ELEV } \\
\text { FT }\end{array}$ & $\begin{array}{c}\text { SAND } \\
\text { F T }\end{array}$ & $\begin{array}{l}\text { FUP } \\
\text { FLEV }\end{array}$ & $\begin{array}{c}\text { SAND } \\
\text { FT }\end{array}$ & $\begin{array}{l}---=1 \\
\text { TUP } \\
\text { ELEV } \\
\text { FT }\end{array}$ & $\begin{array}{c}\text { SAND } \\
\text { FT }\end{array}$ & $\begin{array}{l}\text { WELL } \\
\text { DEPTH }\end{array}$ & $\begin{array}{c}\text { TEMP } \\
F\end{array}$ & $\begin{array}{l}A \\
b \\
E\end{array}$ \\
\hline & & & 3204 & 30 & 3000 & 25 & $\begin{array}{l}1709 \\
2838 \\
2949\end{array}$ & $\begin{array}{l}75 \\
66\end{array}$ & $\begin{array}{l}1446 \\
1495 \\
1821 \\
2565 \\
2706\end{array}$ & $\begin{array}{l}120 \\
125 \\
110 \\
150 \\
120\end{array}$ & $\begin{array}{l}1275 \\
1365 \\
1681 \\
2358 \\
2564\end{array}$ & $\begin{array}{l}0 \\
0\end{array}$ & $\begin{array}{l}1219 \\
1350 \\
1669 \\
2345 \\
2482\end{array}$ & $\begin{array}{l}30 \\
40 \\
30 \\
35 \\
50\end{array}$ & $\begin{array}{l}1171 \\
2302 \\
2425\end{array}$ & 5 & 2339 & 55 & 2164 & 85 & $\begin{array}{l}4175 \\
2528 \\
2151 \\
2856 \\
2553\end{array}$ & $\begin{array}{l}112 \\
110 \\
116\end{array}$ & $\begin{array}{l}\mathrm{J} \\
\mathrm{t}\end{array}$ \\
\hline & & & & & 3262 & 20 & $\begin{array}{l}2422 \\
3004 \\
2774 \\
3215\end{array}$ & $\begin{array}{l}68 \\
60 \\
65 \\
60\end{array}$ & $\begin{array}{l}2169 \\
2767 \\
2515 \\
2940 \\
2772\end{array}$ & $\begin{array}{l}130 \\
120 \\
120 \\
105 \\
120\end{array}$ & $\begin{array}{l}2027 \\
2619 \\
2335 \\
2820 \\
2629\end{array}$ & $\begin{array}{l}0 \\
0\end{array}$ & $\begin{array}{l}2012 \\
2007 \\
2807 \\
2613\end{array}$ & $\begin{array}{l}45 \\
90\end{array}$ & $\begin{array}{l}1965 \\
2517 \\
2759 \\
2559\end{array}$ & $\begin{array}{l}25 \\
40\end{array}$ & $\begin{array}{l}2667 \\
2416\end{array}$ & $\begin{array}{l}0 \\
0\end{array}$ & $\begin{array}{l}2487 \\
2244\end{array}$ & $\begin{array}{l}85 \\
70\end{array}$ & $\begin{array}{l}1917 \\
2105 \\
1238 \\
2270 \\
4878\end{array}$ & $\begin{array}{l}93 \\
93 \\
61 \\
85\end{array}$ & $\begin{array}{l}\mathrm{J} \\
\mathrm{J} \\
\mathrm{K} \\
\mathrm{T}\end{array}$ \\
\hline & & & & & & & & & & & & & -3046 & 80 & & & 1728 & 5 & 1017 & 80 & 7050 & & T \\
\hline
\end{tabular}




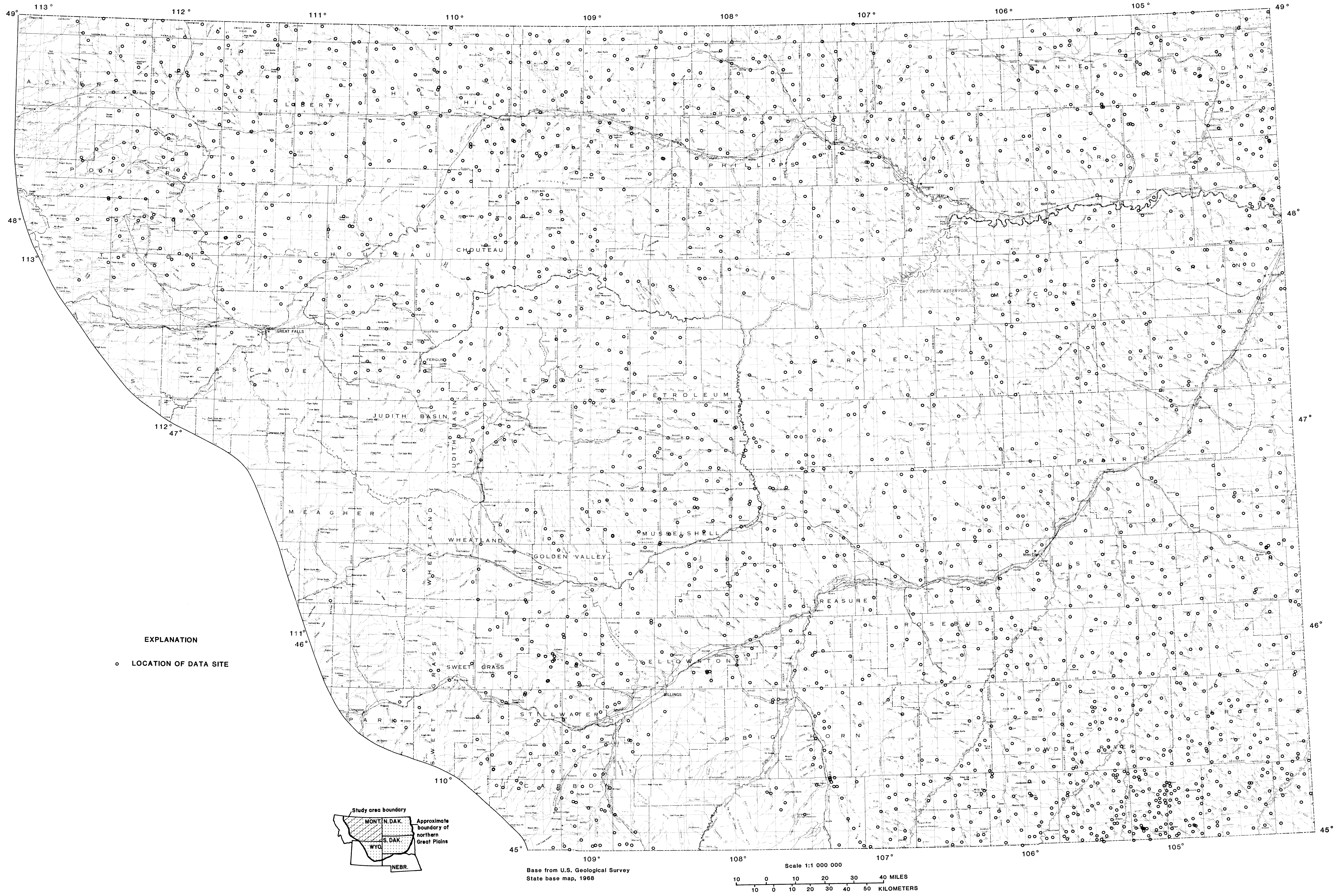

